BNL 50595 Vol. II

(Particle Accelerators and

High-Voltage Machines - TID-4500)

\title{
Reference Documents for the Proposal for a NATIONAL SYNCHROTRON LIGHT SOURCE
}

\author{
Edited by: JOHN P. BLEWETT
}

Prepared for Submission to the

DIVISION OF PHYSICAL RESEARCH

U.S. ENERGY RESEARCH AND DEVELOPMENT ADMINISTRATION

BROOKHAVEN NATIONAL LABORATORY ASSOCIATED UNIVERSITIES, INC.

UNDER CONTRACT NO, EY-76“C.02-0016 WITH THE

UNITED STATES ENERGY RESEARCH AND DEVELOPMENT ADMINISTRATION 


\section{NOTICE}

This report was prepared as an account of work sponsored by the United States Government. Neitber the United States nor the United States Energy Research and Development Administration, nor any of their employees, nor any of their contractors, subcontractors, or their employees, makes any warranty, express or implied, or assumes any legal liability or responsibility for the accuracy, completeness or usefulness of any information, apparatus, product or process disclosed, or represents that its use would not infringe privately owned rights.

Printed in the United States of America

Available from

National Technical Information Service

U.S. Department of Commerce

5285 Port Royal Road

Springfield, VA 22161

Price: Printed Copy $\$ 6.75$; Microfiche $\$ 3.00$

February 1977

675 copies 


\section{Volume II}

\section{Table of Contents}

\section{Section}

1

"Spectra and Optics of Synchrotron Radiation" by $G_{0} K_{\text {. }}$ Green, (April 15, 1976) (a summary of this document is included as Chapter 2 of volume 1 ).

"The Synchrotron Radiation Source" by G. K. Green.

3

"A Short Period Helical Wiggler as an Improved Source of Synchrotron Radiation" by Brian M. Kincaid.

4

"Orbits and Fields in the Helica1 Wiggler" by John P. Blewett and $R_{0}$ Chasman.

(The last two references relate to the material in Chapter 5 of Volume I.) 


\author{
SPECTRA AND \\ OPTICS OF : SYNCHROTRON RADIATION \\ G.K. Green \\ Apri1 15, 1976
}

\title{
ACCELERATOR DEPARTMENT
}

B ROOKHAVEN NATIONAL LA B OR A T ORY A S S OC I A TED UNIVERSITIES, INC. U P T O N, N E W Y O R K 11973 under contract No. $E(30-1)-16$ with the UNITED STATES ENERGY RESEARCH AND DEVELOPMENT ADMINISTRATION 



\section{ABSTRACT}

Sect. I The spectra, angular distribution and polarization functions of synchrotron radiation are tabulated in parametric form. Numerous graphs of the functions are included, and can be used for rapid estimation of photon flux as a function of the various parameters.

Sect. II The extended synchrotron radiation source is described and the exact, but unintegrable, equations are derived. Properties of this source depend upon at least nine parameters. An approximation of the source accurate enough for estimating flux in optical instruments is developed.

Sect. III power and power density in the radiation beam are described and convenient approximations are developed.

Sect. IV Simple optical transformations are used to illustrate some of the important properties of the extended source described in sect. II.

Appendix A Brief description and short table of the Bessel functions used.

Appendix 13 Outline of the properties of electron orbits in a storage ring.

Appendix $C$. Description and short table of integrating function ef $(a, Y)$. 


\section{Synchrotron Radiation Spectra}

The basic equations of synchrotron radiation were published by Schwinger and by Sokolov and Ternov. Quantitative measurements of the radiation were given by Tomboulian and Hartman. 3 However, there is no reasonably complete numerical compendium of the spectra in the literature, and the required Bessel functions are not easily available in tables. (They are now readily generated by the large scientific computers.) Numerous review papers have been published, of which Mack, ${ }^{4}$ Codling, ${ }^{5}$ Rowe ${ }^{6}$ and the orsay Group are good examples. The CEA internal report by Mack is parametric in terms of flux pex ev but is not now easy to obtain (see Appendix A). The Orsay Group paper has the most complete published survey of the spectra but the many graphs and tables apply specifically to the ACO and DCI rings. If one can find it (B.M. Rowe kindly sent me a copy) there is a useful report by Ellis and Stevenson ${ }^{8}$ which tabulates $K_{1 / 3}, K_{2 / 3}, K_{5 / 3}$ and $G(x)$.

This section is an attempt to summarize the characteristics of synchrotron radiation in parametric form. Tables and graphs are arranged to give values to two or three figures by inspection, or by use of a hand calculator.

\section{Fundamental Equations}

If an electron is moving with velocity $v$ the quantity $\beta$ is defined by

$$
B=v / c
$$

and the total energy of the electron is

$$
E=\frac{m_{o} c^{2}}{\sqrt{1-\beta^{2}}}=\gamma m_{o} c^{2}
$$

with $n_{0}$ the electron rest mass and $c$ the velocity of light. Since the rest energy of the electron is $0.5110 \mathrm{MeV}$

$$
Y=1957 \mathrm{E}_{\mathrm{GeV}}
$$

and at large values of $\gamma$ we can put $E$ as either total energy or kinetic energy with very small error. (Particle energy in accelerators is usually quoted as kinetic energy).

Since

$$
B=\left(1-1 / \gamma^{2}\right)^{\frac{1}{2}}=1-1 / 2 \gamma^{2}
$$


and even at $\gamma=1000$ is less than 1 by only $1 / 2$ part per million, $\beta$ will be set equal to 1 and will not be explicitly included in the equations.

If the electron is moving in a circular path in magnetic field $B$ the radius of curvature is

$$
p=p c / e B=m_{o} B \gamma c^{2} / e B
$$

and the energy radiated per turn is (Ref. 1, Eq. I.10)

$$
\delta \mathrm{E}=4 \pi e^{2} \gamma^{4} / 3 p
$$

Schwinger (Eq. II.16) derives the power per unit angular frequency of the radiation, $\omega$, per electron, per unit time

$$
P(\omega, t)=\frac{3^{3 / 2} e^{2}}{4 \pi \rho} \gamma^{4} \frac{\omega_{0}^{\omega}}{2} \int_{c / \omega}^{\infty} k_{5 / 3}(\eta) d \eta
$$

with

$$
\omega_{0}=c / p \text { and } \omega_{c}=\frac{3}{2} \omega_{0} \gamma^{3}=3 c \gamma^{3} / 2 p
$$

Using $\frac{d X}{d w}=\frac{d X}{d v} \frac{d v}{d w}$ and the relationships

$$
\begin{aligned}
& \omega=2 \pi \nu=2 \pi c / \lambda \\
& \lambda_{c}=2 \pi c / \omega_{c}=4 \pi \rho / 3 \gamma^{3} \\
& \lambda=\left(\lambda / \lambda_{c}\right) 4 \pi \rho / 3 \gamma^{3} \\
& \varepsilon=h \nu=h \omega / 2 \pi=h c / \lambda \\
& \omega / \omega_{c}=\nu / \nu_{c}=\varepsilon / \varepsilon_{c}=\lambda_{c} / \lambda=y
\end{aligned}
$$

and defining the resolution by $k=\Delta \lambda / \lambda$; the power $P$ and number of photons per $\sec \mathrm{N}$ radiated into all space per electron are:

$$
\begin{array}{ll}
P(\lambda, t)=\frac{3^{5 / 2}}{16 \pi^{2}} \frac{e c \gamma^{7}}{\rho^{3}}\left(\frac{\lambda}{\lambda}\right) \int_{y}^{3} K_{5 / 3}^{\infty}(\eta) d \eta & \text { per unit } \lambda \\
N(\lambda, t)=\frac{3^{3 / 2}}{4 \pi} \frac{e^{2} \gamma^{4}}{h \rho^{2}}\left(\frac{\lambda}{\lambda}\right) \int_{y}^{2} k_{5 / 3}(\eta) d \eta & \text { per unit } \lambda \\
N_{k}(\lambda, t)=3^{\frac{1}{2}} \frac{k^{2} y}{h \rho}\left(\frac{\lambda}{\lambda}\right) \int_{y}^{\infty} k_{5 / 3}(\eta) d \eta \quad \text { per } k \lambda
\end{array}
$$

\footnotetext{
cgs units are used in accordance with Refs. 1 and 2.
} 


$$
N_{\Delta \varepsilon}(e, t)=\frac{4 \pi}{3^{\frac{1}{2}}} \frac{\mathrm{e}^{2} \Delta \varepsilon}{\mathrm{h}^{2} \mathrm{\gamma}^{2}} \int_{\mathrm{y}}^{\infty} \mathrm{K}_{5 / 3}(\eta) \mathrm{d} \eta \quad \text { per } \Delta \varepsilon
$$

The ring current in amperes is related to the number of electrons and to the revolution frequency by

$$
\frac{I}{10}=\mathrm{n} \frac{\epsilon}{\mathrm{C}}=\frac{\mathrm{ne}}{2 \mathrm{~T} \rho}
$$

(In a ring with field free sections the circumference $c$ is greater than $2 \pi \rho$ and $I / 10=$ ne/C. But the number radiating is the fraction $2 \pi p / \mathrm{C}$ of the total so $n=2 \pi \rho l / 10$ e in agreement with (7)). If the formuli of (6) are multiplied by $n$ and by $\theta / 2 \pi$ the result is radiation in all vertical angles per current $\mathrm{T}$ and arc $\theta$;

$$
\begin{aligned}
& p(\lambda, t)=\frac{3^{5 / 2}}{160 \pi^{2}} \frac{e c \theta I y^{7}}{p^{2}}\left(\frac{\lambda c}{\lambda}\right) \int_{y}^{3} K_{5 / 3}^{\infty}(\eta) d \eta \quad \text { per unit } \lambda \\
& N(\lambda, t)=\frac{3^{3 / 2}}{40 \pi} \frac{e \theta T \gamma^{4}}{h p}\left(\frac{\lambda}{\lambda}\right)^{2} \int_{y}^{\infty} k_{5 / 3}(\eta) d \eta \quad \text { per unit } \lambda \\
& N_{k}(\lambda, t)=\frac{3^{\frac{1}{2}}}{10} \frac{\operatorname{ke\theta } \eta y}{h}\left(\frac{\lambda c}{\lambda}\right) \int_{y}^{\infty} K_{5 / 3}(\eta) d \eta \quad \text { per } k \lambda \\
& N_{\Delta \varepsilon}(\varepsilon, t)=\frac{4 \pi}{10 \cdot 3^{\frac{3}{2}}} \frac{e \Delta \epsilon \theta t}{h^{2} c} \frac{\rho}{\gamma^{2}} \int_{y}^{\infty} k_{5 / 3}(\eta) d \eta \quad \text { per } \Delta \varepsilon \\
& \text { or } \quad=\frac{3^{\frac{3}{2}}}{10} \frac{e \Delta \epsilon \theta \mathrm{I}}{h^{2} c} \gamma \lambda_{c} \int_{y}^{\infty} K_{5 / 3}(\eta) d \eta \quad \text { per } \Delta \epsilon
\end{aligned}
$$

The power radiated at al1 wavelengths as a function of angle $\psi$ to the orbit is (Ref. 1, la. IT.36)

$$
\begin{aligned}
P(\psi, t) & =\frac{c e^{2}}{\rho^{2}} \gamma^{5}\left\{\left(1+\gamma^{2} \psi^{2}\right)^{-5 / 2}\left[\frac{7}{16}+\frac{5}{16} \frac{\gamma^{2} \psi^{2}}{1+\gamma^{2} \psi^{2}}\right]\right\} \\
\text { or } P(\psi, t) & =\frac{\operatorname{ce} \theta I}{10 \rho} \gamma^{5} \mathrm{~F}(\gamma \psi) \text { per rad } \psi \quad \text { ergs } / \mathrm{sec}
\end{aligned}
$$

\footnotetext{
*I in amps, $\theta$ in radians, $\rho$ in $\mathrm{cm}$, other units see $\mathrm{p} .7$.
} 


\section{Angular and Polarization Functions}

Synchrotron radiation is normally generated over an orbital arc $\theta$ much larger than the radiation angle of emission, and is usually collected in such a manner as to sum over the angles in the orbital plane. We are then justified in taking the radiation from arc $\theta$ as $\theta / 2 \pi$ times the total from a complete circular orbit. The variation with angle $\psi$, relative to the orbital plane, is directly observable and is a complex function of $\gamma$, $\rho, \lambda / \lambda_{c}$ and $\psi$.

Sokolov and Ternov ${ }^{2}$ have examined the polarization in considerable detail. They compute that, if $\beta \approx 1$, the fl polarized component of the radiation (electric vector parallel to the orbital plane) contains $7 / 8$ of the total radiated power, and the 1 component on $1 y 1 / 8$. The proportion varies with wavelength. If $W$ is the energy (per sec) radiated into all angles then (Ref. 2, p. 32)

$$
\begin{aligned}
& d_{i}=w_{i}(y) d y \quad w i t h y=\lambda_{c} / \lambda=e / e_{c} \\
& \varphi_{i}(y)=\frac{9 \sqrt{3}}{16 \pi} \text { y }\left\{\left(l_{2}^{2}+\ell_{3}^{2}\right) j_{y}^{\infty} k_{5 / 3}(\eta) d \eta+\left(l_{2}^{2}-l_{3}^{2}\right) k_{2 / 3}(y)\right\}
\end{aligned}
$$

$\ell_{2}=1, \ell_{3}=0$ for $\|$. Linear polarization; $\ell_{2}=0, \ell_{3}=1$ for 1 Linear polarization. If we could observe the radiation from a short segment of arc, $\Delta \theta \ll 1 / \gamma$, the tota $1 \perp$ component of radiation would appear to be four lobes, the axis of each lying at angle $1 / 2 \gamma$ relative to the orbital plane, and $1 / 2 \gamma$ relative to the normal plane through the tangent. Since only the most critical optics could resolve the $\theta$ angular structure, it is convenient to express the functions of $\psi$ as an average over $\theta$.

The power radiated by a single electron in solid angle $d \Omega$ is given by Sokolov and Ternov ${ }^{2}$ (Eq. 5.17)

$$
\mathrm{dP}_{i}(\theta, \nu)=\frac{c e^{2}}{\rho^{2}} \frac{\nu^{2}}{6 \pi^{3}}\left\{l_{2} \in \mathrm{K}_{2 / 3}\left(\frac{\psi}{3} e^{2 / 3}\right)+l_{3} \cos \theta \sqrt{\varepsilon} \mathrm{K}_{1 / 3}\left(\frac{\nu}{3} \varepsilon^{3 / 2}\right)\right\}^{2} \mathrm{~d} \Omega
$$

in which their $\theta$ is the complement of $\psi$. Harmonic order $v$ defines $\omega=v_{o}=v_{c} / \rho$

$$
\omega_{c}=\frac{3 \omega}{2} \gamma^{3} \quad \varepsilon=1-\beta^{2} \sin ^{2} \theta \div \frac{1+\gamma^{2} \psi^{2}}{\gamma^{2}}
$$


and $l_{2}=1, l_{3}=0$ for $\| ; l_{2}=0, l_{3}=1$ for polarization. If we set $\frac{\mathrm{dP}}{\mathrm{d} \psi}=2 \pi \frac{\mathrm{dP}}{\mathrm{d} \Omega}$ and substitute for $\psi$ and $\omega$

$$
\begin{aligned}
& \frac{d P}{d \psi}=\frac{3 e^{2}}{4 \pi^{2}}\left(\frac{\omega}{\omega_{c}}\right)^{2} \gamma^{2}\left\{\left(1+\gamma^{2} \psi^{2}\right)\left[\ell_{2} \mathrm{~K}_{2 / 3}(\xi)+\ell_{3} \gamma \psi\left(1+\gamma^{2} \psi^{2}\right)^{-\frac{1}{2}} \mathrm{~K}_{1 / 3}(\xi)\right\}^{2}\right. \\
& \xi=\frac{\omega}{2 w_{c}}\left(1+\gamma^{2} \psi^{2}\right)^{3 / 2}=\frac{\lambda}{2 \lambda}\left(1+\gamma^{2} \psi^{2}\right)^{3 / 2}
\end{aligned}
$$

or $\frac{\mathrm{dP}}{\mathrm{d} \psi}=\frac{3 \mathrm{e}^{2}}{4 \pi^{2} \rho}\left(\frac{\omega}{\omega}\right)^{2} \gamma^{2} \mathrm{~F}\left(\lambda_{c} / 2 \lambda, \gamma \psi\right)$

If the two components are summed this equation is identical with schwinger's angular distribution equation I I.34. Making the substitutions of the previous section gives the radiation per I amperes, arc $\theta$ and per radian of 1 ;

$$
\begin{aligned}
& P(\psi, \lambda, t)=\frac{27}{320 \pi^{3}} \frac{\operatorname{ec} \theta I Y^{8}}{p^{2}}\left(\frac{\lambda_{c}}{\lambda}\right) F\left(\lambda_{c} / 2 \lambda, \gamma \psi\right) \text { per unit } \lambda \\
& N(\psi, \lambda, t)=\frac{9}{80 \pi^{2}} \frac{e \theta T \gamma^{5}}{h \rho}\left(\frac{\lambda c}{\lambda}\right)^{3} T \quad \therefore \text { per unit } \lambda \\
& N_{k}(\psi, \lambda, t)=\frac{3 k e \theta I \gamma^{2}}{20 \pi h}\left(\frac{\lambda}{\lambda}\right)^{2} F \quad \text { per } k \lambda
\end{aligned}
$$

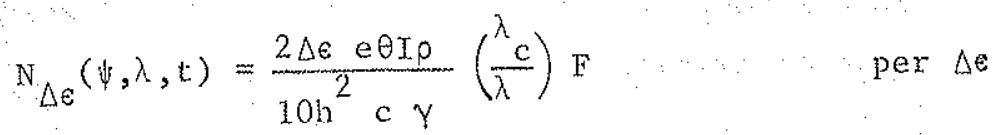

$$
\begin{aligned}
& N_{\Delta \varepsilon}(\psi, \varepsilon, t)=\frac{8 \pi}{30} \frac{\Delta e \text { ee } \theta \mathrm{I} \rho^{2}}{h^{3} c^{2} \gamma^{4}} \mathrm{~F} \quad \text { per } \Delta \varepsilon
\end{aligned}
$$

At $\psi=0$

$$
F\left(\lambda_{c} / 2 \lambda, 0\right) \equiv F \|(0)=k_{2 / 3}^{2}\left(\lambda_{c} / 2 \lambda\right)
$$


At angle

$$
\begin{aligned}
& F_{\|}(\psi)=\left(1+\gamma^{2} \psi^{2}\right)^{2} \mathrm{~K}_{2 / 3}^{2}\left[\frac{\lambda_{c}}{2 \lambda}\left(1+\gamma^{2} \psi^{2}\right)^{3 / 2}\right] \\
& F_{\perp}(\psi)=\gamma^{2} \psi^{2}\left(1+\gamma^{2} \psi^{2}\right)^{2} k_{1 / 3}\left[\frac{\lambda_{c}}{2 \lambda}\left(1+\gamma^{2} \psi^{2}\right)^{3 / 2}\right]
\end{aligned}
$$

for convenient normalization set

$$
\begin{aligned}
& N_{\|}(\psi, \lambda)=N_{\|}(0) F_{\|}(\psi) / F_{\|}(0) \\
& N_{\perp}(\psi, \lambda)=N_{\|}(0) F_{\perp}(\psi) / F_{\|}(0) \text { and } N=N_{\|}+N_{\perp}
\end{aligned}
$$

expressed as functions of $\gamma, \lambda_{c} / \lambda$ and $\gamma \psi$

The degree of linear polarization is then

$$
P=\frac{F_{l}-F_{\perp}}{F_{l}+F_{\perp}}
$$

In the orbital plane the polarization is linear and paralle1. Out of the plane it is elliptical; the expressions are given by Sokolov and Ternov. ${ }^{2}$ One rather remarkable feature of this elliptical polarization is a phase difference between components always $\pm \pi / 2$, so that the axes of the polarization ellipse are always $\|$ and $\perp$ to the orbital plane.

\section{Numerical Values}

Both Schwinger and Sokolov and Ternov use cgs units in their equations. Substituting

$$
\begin{aligned}
\mathrm{e} & =4.803 \times 10^{-10} \mathrm{esu} \\
\mathrm{c} & =2.9979 \times 10^{10} \mathrm{~cm} / \mathrm{sec} \\
\mathrm{h} & =6.6256 \times 10^{-27} \mathrm{erg} \mathrm{sec} \\
1 \mathrm{ev} & =1.6021 \times 10^{-12} \mathrm{erg} \\
1 \AA & =10^{-8} \mathrm{~cm}
\end{aligned}
$$

$$
B p=1704 \text { y gauss } \mathrm{cm}=33.35 \mathrm{E}_{\mathrm{GeV}} \text { kgauss-m }
$$


(4) $\quad \delta \mathrm{E}=88.5 \mathrm{E}_{\mathrm{GeV}}^{4 / \mathrm{P}_{\mathrm{m}}}$ kev per turn

(7) $\quad \mathrm{n}=1.308 \times 10^{11} \mathrm{\rho}_{\mathrm{m}} \mathrm{I}_{\mathrm{a}}$ electrons radiating

(5) $\quad \lambda_{\mathrm{c}}=5.59 \rho_{\mathrm{m}} / \mathrm{E}_{\mathrm{GeV}}^{3}=186.4 / \mathrm{B}_{\mathrm{kg}} \mathrm{E}_{\mathrm{GeV}}^{2} \AA$

With $\rho$ in meters, $\lambda$ and $\lambda_{c}$ in $\AA$, $\epsilon$ in $e V, I$ in amps and $\theta$ in radians

$$
\varepsilon_{\mathrm{c}}=2218 \mathrm{E}_{\mathrm{GeV}}^{3} / \rho \mathrm{eV}
$$

(8) $P(\lambda, t)=1.421 \times 10^{-13} \frac{\theta \mathrm{I \gamma}}{\rho^{7}}\left(\frac{\mathrm{c}}{\lambda}\right)^{3} \int_{y=\lambda_{c} / \lambda}^{\infty} \mathrm{k}_{5 / 3}(\eta) \mathrm{d \eta} \operatorname{ergs} / \AA \mathrm{sec}$ all $N(\lambda, t)=2.998 \times 10^{5} \frac{I \theta \gamma^{4}}{\rho}\left(\frac{\lambda c}{\lambda}\right) \int_{y}^{\infty} k_{5 / 3}(\eta) \mathrm{d} \eta$ photons $/ \AA$ sec a $a 11 \psi$

$N_{k}(\lambda, t)=1.256 \times 10^{16} \mathrm{~kJ} \theta \gamma\left(\frac{\lambda}{\lambda}\right) \int_{y}^{\infty} \mathrm{k}_{5 / 3}(\eta) \mathrm{d} \eta \mathrm{ph} / \mathrm{k} \lambda \mathrm{sec}$

$N_{\Delta \varepsilon}(\lambda, t)=4.242 \times 10^{22} \frac{\mathrm{I} \theta \rho}{\gamma^{2}} \int_{y}^{\infty} K_{5 / 3}(\eta) \mathrm{d} \eta$

$$
=1.013 \times 10^{12} \gamma \lambda_{c} \int_{y}^{\infty} k_{5 / 3}(\eta) \mathrm{d} \eta \mathrm{ph} / \mathrm{eV} \mathrm{sec}
$$

(9) $\mathrm{P}=1.440 \times 10^{-15} \frac{y^{5} \mathrm{I}}{\rho} \mathrm{F}(\gamma \psi) \omega / \mathrm{mrad} \theta, \mathrm{mrad} \psi$

(12) $P(\psi, \lambda, t)=3.918 \times 10^{-1.4} \frac{\theta \mathrm{J} \gamma^{8}}{\rho^{2}}\left(\frac{\lambda_{c}}{\lambda}\right)^{4} \mathrm{~F}\left(\lambda_{\mathrm{c}} / 2 \lambda, \gamma \psi\right) \operatorname{ergs} / \AA \sec \mathrm{rad} \psi$

$$
\begin{array}{ll}
\mathrm{N}(\psi, \lambda, t)=8.263 \times 10^{4} \frac{\theta \mathrm{I} \mathrm{Y}^{5}}{\rho}\left(\frac{\lambda_{\mathrm{c}}}{\lambda}\right)^{3} \mathrm{~F} & \mathrm{ph} / \AA \mathrm{Asec} \mathrm{rad} \psi \\
\mathrm{N}_{\mathrm{k}}(\psi, \lambda, \mathrm{t})=3.461 \times 10^{15} \mathrm{k} \theta \mathrm{I} \gamma^{2}\left(\frac{\lambda_{\mathrm{c}}}{\lambda}\right)^{2} \mathrm{~F} & \mathrm{ph} / \mathrm{k} \lambda \mathrm{sec} \mathrm{rad} \psi \\
\mathrm{N}_{\Delta \varepsilon}(\psi, \lambda, t)=1.169 \times 10^{22} \frac{\theta \mathrm{Ig}}{\gamma}\left(\frac{\lambda}{\lambda}\right) \mathrm{F} & \mathrm{ph} / \mathrm{eV} \mathrm{sec} \mathrm{rad} \psi
\end{array}
$$


$\mathrm{N}_{\Delta \varepsilon}(\psi, \varepsilon, t)=3.951 \times 10^{28} \frac{e \theta \mathrm{T} \rho^{2}}{\gamma^{4}} \mathrm{~F} \quad \mathrm{ph} / \mathrm{eV} \mathrm{sec} \mathrm{rad} \psi$

The function $G$ is defined by Tomboulian and Hartman ${ }^{3}$ as

$$
G=y^{3} \int_{y}^{\infty} K_{5 / 3}(\eta) d \eta
$$

This can be generalized to

$$
G_{i}=y^{i} \int_{y}^{\infty} K_{5 / 3}(\eta) d \eta \text { with } i=0,1,2,3
$$

Also let $H_{\mathrm{I}}(y, 0)=\mathrm{y}^{1} \mathrm{~K}_{2 / 3}^{2}(\mathrm{y} / 2)$

We can now write (17) and (19) in power and photons per sec per ma and per mrad of $\theta$ and $y$ (where applicable)

$$
\text { with } \quad y=\lambda_{c} / \lambda=e_{c} / e_{c}
$$

(8) $P(\lambda)=1.421 \times 10^{-19} \frac{y^{7}}{\rho^{2}} G_{3}(y) \operatorname{ergs} / \mathrm{A}, \mathrm{sec}, \mathrm{ma}, \operatorname{mrad} \theta, \mathrm{al1}$

$$
\begin{aligned}
& N(\lambda)=2.998 \times 10^{-1} \frac{y^{4}}{\rho} G_{2}(y) \quad p h / \AA, \sec , \operatorname{ma}, \operatorname{mrad} \theta \text { a } 11 \psi \\
& \mathrm{N}_{\mathrm{k}}(\lambda)=1.256 \times 10^{10} \mathrm{kyG}_{1}(\mathrm{y}) \mathrm{ph} / \mathrm{k} \lambda, \mathrm{sec}, \operatorname{ma}, \operatorname{mrad} \theta \mathrm{a} 11 \psi \\
& N_{\Delta \varepsilon}(\lambda)=4.24 \times 10^{16} \frac{\rho}{\gamma^{2}} G_{0}(y) \\
& =1.013 \times 10^{6} \gamma \lambda_{c} G_{o}(y) \mathrm{ph} / \mathrm{eV}, \mathrm{sec}, \mathrm{ma}, \operatorname{mrad} \theta \text { a } 11 \psi
\end{aligned}
$$

and at $\psi=0$

$$
\text { (12) } \begin{aligned}
\mathrm{P}(\lambda, 0) & =3.918 \times 10^{-23} \frac{y^{8}}{\rho^{2}} \mathrm{H}_{4}(y, 0) \text { ergs } / \AA \text {, sec, ma, mrad } \theta, \operatorname{mrad} \psi \\
\mathrm{N}(\lambda, 0) & =8.263 \times 10^{-5} \frac{y^{5}}{\rho} \mathrm{H}_{3}(y, 0) \mathrm{ph} / \AA \text {, sec, ma, mrad } \theta \text { mrad } \psi \\
\mathrm{N}_{\mathrm{k}}(\lambda, 0) & =3.461 \times 10^{6} \mathrm{ky}^{2} \mathrm{H}_{2}(\mathrm{y}, \mathrm{o}) \mathrm{ph} / \mathrm{k} \lambda, \mathrm{sec}, \mathrm{ma}, \mathrm{mrad} \theta, \operatorname{mrad} \psi
\end{aligned}
$$




$$
\begin{aligned}
& N_{\Delta \epsilon}(\lambda, 0)=1.169 \times 10^{13} \frac{\mathrm{g}}{\gamma} \mathrm{H}_{1}(\mathrm{y}, 0) \mathrm{ph} / \mathrm{eV}, \mathrm{sec}, \operatorname{ma}, \operatorname{mrad} \theta, \operatorname{mrad} \psi \\
& N_{\Delta \varepsilon}(\epsilon, 0)=3.951 \times 10^{19} \frac{\varepsilon_{0}^{2}}{\gamma^{4}} \mathrm{H}_{0}(\mathrm{y}, 0) \mathrm{ph} / \mathrm{eV}, \mathrm{sec}, \mathrm{ma}, \operatorname{mrad} \theta, \operatorname{mrad} \psi
\end{aligned}
$$

of the above functions the ones most useful in optics and spectroscopy are $N_{k}$ and $N_{\Delta e^{*}} N_{k}$, with $k$ small, is directiy related to the resolution of monochromators and spectrometers. $N_{\Delta e}$ can be directly applied to level widths and level density and so seems preferable to $N$ per $\Delta \bullet / c$, although $N_{\Delta e}$ is misleading at small e just as $N$ is misleading at small $\lambda . N(\lambda)$ is the function most often seen in the literature, al though its usefulness is limited to those instruments which have constant rather than proportional. resolution.

The flux functions of (22) and (23) are listed in table I in terms of functions $F_{i}$.. These functions are simply, as a mater of convenience, the numerical constant combined with the Bessel function. A short table of the Bessel functions is included in Appendix A. The functions $F_{0}$ through $F_{6}$ are graphed in Figs. I through 7. For estimating flux it is then only necessary to select the multiplier from Table I and co apply it to the selected point of the corresponding curve. These log-log graphs are convenient because their shape remains fixed while the axes are translated by the multipliers.* $N_{k}(\lambda)$ and $N_{k}(\lambda, 0)$ seem particularly useful because, for a given ratio of $\lambda_{c} / \lambda$, the flux is proportional on $1 y$ to $\gamma$ or to $\gamma^{2}$ respectively. The parameter $\gamma$ has been used, rather than $E$, because the angular functions are expressed more neatly in terms of $\gamma$.

A simpleminded log-log family of $\lambda_{c}$ values is draw in $\mathrm{Figs.} 8$ and 9 for constant $B$ and constant $p$. These tamilies can be scanned for ranges of values, and are handy for sketching "tunjng curves"。

The fraction of power radiated at all wavelengths greater than $\lambda$ is

$$
\int_{\lambda}^{\infty} P(\lambda) d \lambda / P_{\text {tota } l .}
$$

and the numbex of photons at all wavelengths greater than $\lambda$ is

\footnotetext{
This is not true for Tig. 7 or for any function whose multipliers contain $\lambda$ or 6 explicitly,
} 
TABLE I

\section{General Relations}

$$
\begin{aligned}
& \gamma=1957 \mathrm{E}_{\mathrm{GeV}} \\
& \mathrm{BP}_{\mathrm{p}}=1704 \text { Y gauss } \mathrm{cm}=33.35 \mathrm{E} \mathrm{GeV} \text { kgauss }-\mathrm{m} \\
& \delta \mathrm{E}=88.5 \mathrm{E}_{\mathrm{GeV}}^{4} / \mathrm{p}_{\mathrm{m}} \text { kev per turn } \\
& \mathrm{n}=1.308 \times 10^{11} \mathrm{\rho} \mathrm{I} \text { electrons radiating } \\
& \lambda_{c}=5.59 \rho_{\mathrm{m}} / \mathrm{E}_{\mathrm{GeV}}^{3}=186.4 / \mathrm{B}_{\mathrm{kg}} \mathrm{E}_{\mathrm{GeV}}^{2} \AA \\
& \lambda_{c}=4.189 \times 10^{10} \rho_{\mathrm{m}} / \gamma^{3} \AA \\
& \varepsilon=12,398 / \lambda \text { eV, } \AA \\
& \varepsilon_{c}=2218 \mathrm{E}_{\mathrm{GeV}}^{3} / \rho_{\mathrm{m}}=2.960 \times 10^{-7} \mathrm{\gamma}^{3} / \rho_{\mathrm{m}} \mathrm{eV} \\
& y=\lambda_{c} / \lambda=\epsilon / \epsilon_{c}
\end{aligned}
$$

FIux in photons per sec, ma, mrad $\theta$

$$
\begin{aligned}
& N(\lambda)=\gamma^{4} / \rho_{0}\left(\lambda_{c} / \lambda\right) \quad \operatorname{per} \AA, a 11 \psi \\
& N_{k}(\lambda)=\mathrm{k}_{\mathrm{F}} \mathrm{F}_{1}\left(\lambda_{\mathrm{c}} / \lambda\right) \quad \because \operatorname{per} \mathrm{k} \lambda \text {, a } 11 \psi \\
& N_{\Delta \epsilon}(\lambda)=\left(\rho / \gamma^{2}\right) F_{2}\left(\lambda_{c} / \lambda\right) \\
& =\gamma \lambda_{c}{ }_{3}\left(\lambda_{c} / \lambda\right) \quad \cdots \text { per eV, all } \psi \\
& \mathrm{N}_{\mathrm{k}}(\lambda, 0)=\mathrm{kr}^{2} \mathrm{~F}_{4}\left(\lambda_{\mathrm{c}} / \lambda, 0\right) \quad \operatorname{per} \mathrm{k} \lambda, \operatorname{mrad} \psi \text { at } \psi=0 \\
& { }_{\Delta \varepsilon}(\lambda, 0)=(\rho / \gamma) F_{5}\left(\lambda_{c} / \lambda, 0\right) \text { per } e V, \operatorname{mrad} \psi \text { at } \psi=0 \\
& { }^{N}{ }_{\Delta \epsilon}(\epsilon, 0)=\left(\epsilon_{p}^{2} / \gamma^{4}\right) F_{6}\left(\lambda_{c} / \lambda, 0\right) \text { per eV, mrad } \psi \text { at } \psi=0
\end{aligned}
$$




$$
\int_{\lambda}^{\infty} N(\lambda) d \lambda / \int_{0}^{\infty} N(\lambda) d \lambda
$$

These two functions are plotted in Figs. 10 and 11 as $\%$ vs $\lambda / \lambda_{c}$.

The power radiated (at a11 $\lambda$ ) as a function of $\psi$ is derived from (9)

$$
\begin{aligned}
& P(\psi)=1.44 \times 10^{-18}\left(\gamma^{5} / \rho_{\mathrm{m}}\right) \mathrm{F}(\gamma \psi) \text { w/ma, mrad } \theta, \mathrm{mrad} \psi \\
& \text { or } P(\psi)=\left(\gamma^{5} / \rho\right) F_{7}(\gamma \psi)
\end{aligned}
$$

with $F(\gamma \psi)$ and $F_{7}$ plotted in Fig. 12 .

tinear polarization components vary with wavelength. The function of (10)

$$
\varphi_{i}(y)=0.3101 \text { y }\left\{\left(\ell_{2}^{2}+\ell_{3}^{2}\right) G_{0}(y)+\left(\ell_{2}^{2}-l_{3}^{2}\right) k_{2 / 3}(y)\right\}
$$

is plotted in Fig. 13 for the $\|$ component $\left(l_{2}=1, l_{3}=0\right)$, the 1 component $\left(l_{2}=0, \ell_{3}=1\right)$, and the tota 1 , vs. $\lambda / \lambda_{c}=1 / y$. The percentages of $\|$ and of $\perp$ components radiated into all angles are plotted in Fig. 14.

At a given wavelength $\lambda$ the flux varies with vertical angle $\psi$ (13) as:

$$
\begin{aligned}
& F_{\|}(\psi)=\left[1+(\gamma \psi)^{2}\right]^{2} \mathrm{k}_{2 / 3}^{2}\left\{\frac{c}{2 \lambda}\left[1+(\gamma \psi)^{2}\right]^{3 / 2}\right\} \\
& F_{\perp}(\psi)=(\gamma \psi)^{2}\left[1+(\gamma \psi)^{2}\right] \mathrm{k}_{1 / 3}^{2}\left\{\frac{c}{2 \lambda}\left[1+(\gamma \psi)^{2}\right]\right.
\end{aligned}
$$

These functions are plotted in $F i g .15$ as percentage of $F(0)$ vs $\gamma \psi$ for $\lambda / \lambda_{c}$ ratios ranging from 0.2 to 100 . The sum, or variation of total flux vs. $\gamma \psi$, is also shown. Number of photons per sec, ma, mrad $\theta$, mrad $\psi$ at any $\psi$ can then be found by multiplying the ratio of $F i g .15$ by the appropriate $N(\lambda, 0)$.

If the vertical acceptance angle $\Delta \psi$ is very small and is near $\psi=0$ the flux is readily obtained as $\mathrm{N}(\lambda, 0) \Delta \psi$. For larger acceptance angles it is necessary to integrate the angular functions.

* Note that these percentages are functions of $\lambda / \lambda_{\mathrm{c}}$ rather than $\lambda$. 


\section{The Source}

The previous section treated the radiation from a current of electrons as if the current were a filament, or for the angular functions, as if the electrons were travelling on parallel orbits. In an alternating gradient ring the electrons will oscillate about the central orbit and the oscillations will cause a spread in position and angle. This distribution of the beam in size and angle is modulated by the magnetic structure of the ring and the modulation produces a rather rapid variation of the transverse dimensions. Photons radiated by the electrons are distributed in angle about the trajectories of the particles and are emitted all a long the trajectory. Thus we have a source which is extended in three dimensions in configuration space and in six dimensions in phase space. The properties of the source depend upon a sizeable number of parameters. In order to make the problem somewhat manageable the procedures reduce the description of the source to one in four phase space dimensions. There seems to be no very satisfactory general method. An outline of the source in laboratory space provides no angular information. A four-dimensional phase space solution is general, although very complex, but cannot be drawn. We must then use two two-dimensional diagrams. The curvilinear coordinate $s$ is taken along the central electron orbit, assumed to lie in a plane. Transverse coordinate $\mathrm{x}$ is perpendicular to $\mathrm{s}$ and in the orbital plane, and $x^{\prime}=d x / d s$. Since the angles are very small the paraxial approximation $\tan \eta \approx \sin \eta \approx \eta$ is quite good. Similarly, $y$ and $y^{\prime}=d y / d s$ lie in the plane perpendicular to the orbital plane. It is necessary to keep in mind that there are correlations between the $x, x^{\prime}$ plane and the $y, y^{\prime}$ plane and that the figures in these two planes must often be considered together.

The configuration of the electron beam as it goes along $s$ is well known from accelerator orbit dynamics. Emittance of this beam is described by the courant-Snyder invariant. When the electrons in an element ds emit photons the angular spread increases and the resulting photon distribution can then be characterized by an invariant for succeding optical transformations. If the photon distributions from all elements of a source extended in s are transformed to a single plane the result is a planar optical source which can be described by four phase space dimensions. 


\section{Source Derivation}

The electron beam in a well-behaved storage ring will have an elliptical cxoss section in configuration space $x, y$ (Fig. 16). Size of the beam is governed by the characteristics of the ring and by the quantum fluctuations of the emitted radiation. Since the latter are random a stabilized beam (e.g. one with sma11 systematic instabilities) will have a normal distribution with probability density

$$
\left.p(x, y)=\frac{1}{2 \pi \sigma x^{\sigma}} e^{-\left(\frac{x^{2}}{2 \sigma} x^{2}\right.}+\frac{y^{2}}{2 \sigma y^{2}}\right)
$$

The probability functions will be defined as

$$
\begin{aligned}
& P(z)=\frac{1}{\sigma \sqrt{2 \pi}} e^{-\frac{z^{2}}{2 \sigma^{2}}} \\
& \operatorname{erf}(x)=\frac{2}{\sqrt{\pi}} \int_{0}^{x} e^{-t^{2}} d t ; \operatorname{erf}(x / \sigma \sqrt{2})=\frac{1}{\sigma \sqrt{2 \pi}} \int_{-x}^{x} e^{\frac{2}{2 \sigma^{2}} d z} \\
& \frac{2}{\sqrt{\pi}} \int_{0}^{\infty} e^{-t^{2}} d t=\sqrt{\frac{2}{\pi}} \frac{1}{\sigma} \int_{0}^{\infty} e^{-\frac{z^{2}}{2 \sigma^{2}} d z=1} \\
& \frac{P(o)}{\operatorname{erf} \infty}=\frac{1}{\sqrt{2 \pi} \sigma}
\end{aligned}
$$

and to normalize

The current in element $\mathrm{dx} d y$ at $\mathrm{x}, \mathrm{y}$ is

$$
d^{2} I=I_{0} P(x, y) d x d y
$$

Electrons are distributed in phase space $\mathrm{x}, \mathrm{x}^{\prime}$ and $\mathrm{y}, \mathrm{y}^{\prime}$, and typical examples are shown in Fig. 16. Within the slice dy there is distribution in $y^{\prime}$ $\left(y^{\prime}=\mathrm{dy} / \mathrm{ds}\right.$ is the angle of the electron trajectory to the central axis $\left.\mathrm{s}\right)$. Consider the electron in element dydy'. On succesive transits of the ring it will take various positions indicated by the dotted ellipse in Fig. 16, provided the motion is not on a resonance. A resonance is obviously unallowable in a storage ring. These paths of all elements in the phase 
plane are similar and, if y is a normal variate, then y' is also a norma variate. A brief description of particle dynamics of a storage ring is given in Appendix $B$.

An outline of the beam cross section in the y, y plane as we proceed along s can be described by the courant-Snyder invariant

$$
\gamma y^{2}+2 \alpha y y^{2}+\beta y^{2}=\mathrm{E}
$$

This is an ellipse with coefficients $\alpha, \beta, \gamma$ which are functions of $s$ and an area equal to $T$ times the emittance $\mathrm{E}$, a constant of the motion. There are constraints

$$
\begin{gathered}
\beta \gamma=1+\alpha^{2} \\
\alpha=-\beta^{2} / 2
\end{gathered}
$$

Figure 17a shows the beam envelope at a waist, or minimum of $\beta$, where we wi11 set $s=0$. Since $\alpha=0$ at this $s=0$ the emittance ellipse is

$$
\begin{aligned}
& \gamma y^{2}+\beta y^{2}=E \\
& \hat{y}=\sqrt{E / \gamma}, \hat{y}^{\prime}=\sqrt{E / \beta}, \beta \gamma=1
\end{aligned}
$$

It is preferable to express the maxima as

$$
\hat{y}=\sqrt{E \beta}, \quad \hat{y}^{\prime}=\sqrt{E \gamma} \text { and } \hat{y} / \hat{y}^{\prime}=\beta
$$

(The third relation is not true if the ellipse axes are tilted.)

If the ellipse of $\mathrm{Fig}$. $17 \mathrm{a}$ represents the one $\sigma$ contour of $\mathrm{P}\left(\mathrm{y}, \mathrm{y}^{\prime}\right)$ the one $\sigma$ emittance becomes $\sigma_{y} \sigma^{\prime}$, and the ellipse is described by

$$
\frac{\sigma_{y^{\prime}}}{\sigma_{y}} y^{2}+\frac{\sigma_{y}}{\sigma_{y}} y^{\prime 2}=\sigma_{y} \sigma_{y^{\prime}}
$$

At $s$ the transverse coordinates $y, y^{\prime}$ are transformed by

$$
\left|\begin{array}{l}
y_{1} \\
y_{1}^{\prime}
\end{array}\right|=M(0 / s)\left|\begin{array}{c}
y \\
y^{\prime}
\end{array}\right|
$$


and if there is no (magnetic) focussing the transformation matrix is

$$
M(0 / s)=\left|\begin{array}{lll}
1 & s \\
0 & & 1
\end{array}\right|
$$

Since synchrotron 1ight sources are almost always bending magnets without gradient focussing, this matrix can be used to transform $y, y^{\prime}$ and $x, x^{\prime}$ along s rather than the more complex matrices of magnetic focussing sys tems. The one $\sigma$ contour at $s(\mathrm{Fig}$. 17b) is now

$$
\frac{\sigma y^{\prime}}{\sigma y} y^{2}-2 \frac{\sigma}{\sigma} y_{y} y^{\prime}+\left(\frac{y^{\prime}}{\sigma} y^{\prime}+\frac{y^{\prime s}}{\sigma}\right) y^{\prime 2}=\sigma y^{\prime}
$$

If a bivariate distribution is described by (Ref. 9, Sect, 26.3)

$$
-\frac{1}{2\left(1-r^{2}\right)}\left(\frac{x^{2}}{\sigma^{2}}-\frac{2 r x y}{\sigma x y}+\frac{y^{2}}{\sigma^{2}}\right)
$$

the one $\sigma$ contour is

$$
\frac{x^{2}}{\sigma_{x}^{2}}-\frac{2 x x y}{\sigma \sigma y}+\frac{y^{2}}{\sigma_{y}^{2}}=\left(1-r^{2}\right)
$$

and

$$
\iint_{A} P(x, y) d x d y=1-e^{-\frac{1}{2}}=0.39
$$

\begin{tabular}{|c|c|}
\hline $\mathfrak{n}$ & integral \\
\hline 1 & 0.39 \\
\hline 2 & 0.865 \\
\hline 3 & 0.989 \\
\hline 4 & 0.9997 \\
\hline
\end{tabular}

where the integral is taken over the area enclosed by (35). If the contour represents no the integral value is a $^{-\frac{n^{2}}{2}}$ 
A general elliptical contour (centered on the axes) is described by

$$
g x^{2}+2 a x y+b y^{2}=1
$$

and by equating coefficients to (34) or (35)

$$
\begin{aligned}
& r^{2}=a^{2} / b g \quad\left(1-r^{2}\right)=\left(b g-a^{2}\right) / b g \\
& \sigma_{x}^{2}=b g / g\left(b g-a^{2}\right) \quad \sigma_{y}^{2}=b g / b\left(b g-a^{2}\right) \\
& \operatorname{area} / \pi=1 / \sqrt{\mathrm{bg}-\mathrm{a}^{2}}
\end{aligned}
$$

The area is invariant under a linear transformation and it is often useful to set area/m = E, the "emittance" of the beam. Figure 18 1ists several useful properties of (37) with the rh side replaced by $\mathrm{E}$. If (bg-a ${ }^{2}$ ) is set equal to 1 (as is usually done in accelerator dynamics) the maxima of the ellipse are simply described

$$
\hat{x}=\sqrt{\mathrm{Eb}} \quad \hat{\mathrm{y}}=\sqrt{\mathrm{Eg}}
$$

Rewrite (33) with 1 on the rh side and (38) gives for the electron beam at $s$ :

$$
\begin{aligned}
& \sigma_{y_{1}}^{2}=\sigma_{y}^{2}+s^{2} \sigma_{y}^{2} \\
& \sigma_{y_{1}^{2}}^{2}=\sigma_{y^{\prime}}^{2} \\
& \left.r_{1}^{2}=s^{2} \sigma^{2} / / \sigma_{y}^{2}+s^{2} \sigma_{y}^{2}\right) \\
& \left(1-r_{1}^{2}\right)=\sigma_{y}^{2} /\left(\sigma_{y}^{2}+s^{2} \sigma_{y}^{2}\right)
\end{aligned}
$$

while (34) becomes

$$
P_{1}\left(y, y^{\prime}\right)=\frac{1}{2 \pi \sigma \sigma_{y} y^{\prime}} e^{-\frac{1}{2 \sigma_{y}^{2}}\left[y^{2}-2 s y y^{\prime}+\left(\sigma_{y}^{2}+s^{2} \sigma_{y}^{2}\right) y^{\prime \prime}\right]}
$$

To obtain the total current in dy at y (Fig. 16) integrate (27) over all $x$

$$
\mathrm{dI}=\mathrm{I}_{0} P(y) d y
$$




\section{$1-18$}

and since $\quad I_{0} \int^{\infty} P(y) d y=I_{0}$

renormalize $d^{2} I=I_{0} P_{1}\left(y, y^{\prime}\right) d y d y^{\prime}$

When an electron at $y, y$ radiates its position $y$ will not change instantaneously but the radiation will be distributed about its instantaneous direction according to

$$
N_{k \|}(\psi, \lambda)=\frac{3 k e I d s}{20 m \rho h} \gamma^{2}\left(\frac{c}{\lambda}\right)^{2}\left\{\left(1+\gamma^{2} \psi^{2}\right)^{2} k_{2 / 3}^{2}\left[\frac{c}{2 \lambda}\left(1+\gamma^{2} \psi^{2}\right)^{3 / 2}\right]\right\} \Delta \psi
$$

obtained from (12) and (13) by setting $I \theta=I d s / p$ to give number of photons per amp in the element ds. $N_{k} l l$ is somewhat arbitarily selected to simplify the expressions, and because the ll component is reflected or diffracted most efficiently with vertical dispersion. If we define the radiation angle as $Y^{\prime}$ then $\psi=y^{\prime}-y^{\prime}$ and the number of photons from ds in $\mathrm{dY}^{\prime}$ from electron current in dy dy is

$$
d^{4} N=I_{0} P_{1}\left(y, y^{\prime}\right) \frac{N_{k} \|}{I}\left(Y^{\prime}-y^{\prime}, \lambda\right) d y d y^{\prime} d Y^{\prime}
$$

The number of photons in $\mathrm{dY}^{\prime}$ at $\mathrm{Y}^{\prime}$ as a function of $\mathrm{y}$ is

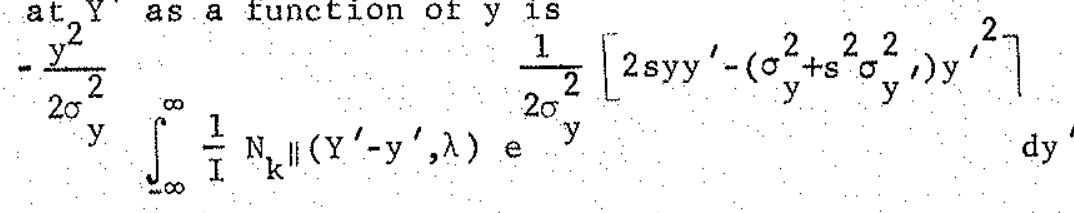

for a given value of $\gamma$ and $\lambda_{c} / \lambda$.

An optical system can be represented by an optical transformation. If a source is extended along the optical axis the elements of the source can be successively transformed into a surface in the image space in order to compute the image. However, if $M\left(s_{i} / s_{j}\right)$ is the transformation along. the optical axis from $s_{i}$ to $s_{j}$ then

$$
M\left(s_{0} / s_{N}\right)=M\left(s_{N-1} / s_{N}\right) \cdots M\left(s_{0} / s_{1}\right)
$$


Then if $M\left(s_{1} / s_{N}\right)$ transforms a surface in the object space to the desired surface in the inage space we can transform all the elements of the orbit to $s_{1}$ and create a synthesized object. This can then be trans ferred to the image space by a single transformation. The synchrotron light source is extended in the direction of the optical axis and it is reasonable to transform all the elements of the source to the transverse plane at its center. The transformation is

$$
\left|\begin{array}{l}
y_{2} \\
y_{2}^{\prime}
\end{array}\right|\left|\begin{array}{ccc}
1 & -s \\
& & \\
0 & & 1
\end{array}\right|\left|\begin{array}{l}
y_{1} \\
Y_{1}^{\prime}
\end{array}\right|
$$

and the number of photons at $Y^{\prime}$ as a function of $y_{2}$, in $d y_{2} d y^{\prime}$, can be obtained by integrating (4.5) over the range of $\mathrm{s}$. Since $\mathrm{N}_{\mathrm{k}}$ ll was defined
as photons per ds

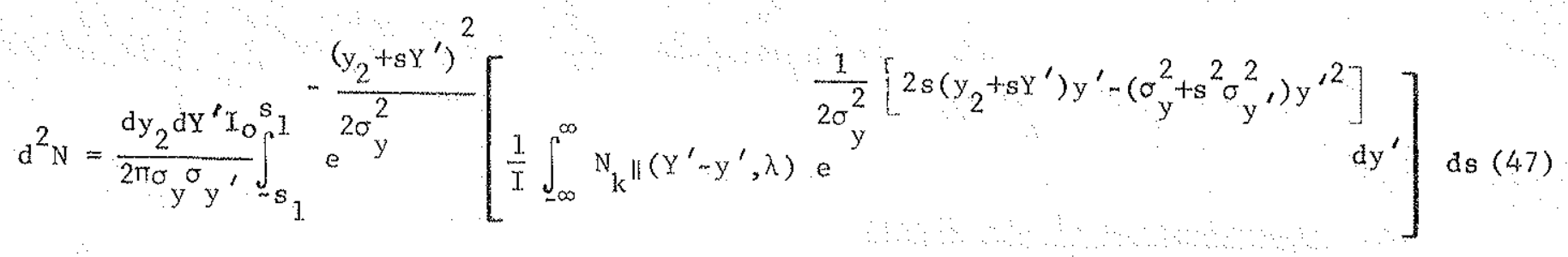

where $y_{2}, Y^{\prime}$ are the $y$ phase coordinates at $s=0$, defined as the position of a waist of the electron beam. This would be the situation at the focus in an insertion. If the source lies between $s_{1}$ and $s_{2}$ and the next waist projected from $s_{1}$ is at $s=0$, the source plane will be at $\vec{s}^{2}=\left(s_{1}+s_{2}\right) / 2$. The equa tion replacing (47) is integrated from $s_{1}$ to $s_{2}$ and is obtained from (45) by the transformation

$$
\left|\begin{array}{cc}
1 & -(s-\tilde{s}) \\
0 & 1
\end{array}\right|
$$

Equation (47) was obtained by assuming that the central orbit lies in the plane $y=0$, a condition reasonably well satisfied when a storage ring is in good adjustment. In the $\mathrm{x}$ phase plane Eq. (45), with $y, Y^{\prime}$ replaced by $x, x^{\prime}$, represents the radiation from arc element ds. But $x X^{\prime}$ are the coordinates relative to $s$ at $\mathrm{d}$. 
The optical axis projects on the plane $s=0$ at (Fig. 19)

$$
x^{2}-s^{2} / 2 p, x=s / p
$$

and the transformation from s to $s=0$ becomes

$$
\left|\begin{array}{ll}
x_{2} \\
x_{2}^{\prime}
\end{array}\right|=\left|\begin{array}{ll}
1 & -s \\
0 & 1
\end{array}\right| x|x|+\left|\frac{s}{2 p}\right|
$$

Eq. (47) expressed in $x, x^{\prime}$ then becomes, projected on $s=0$

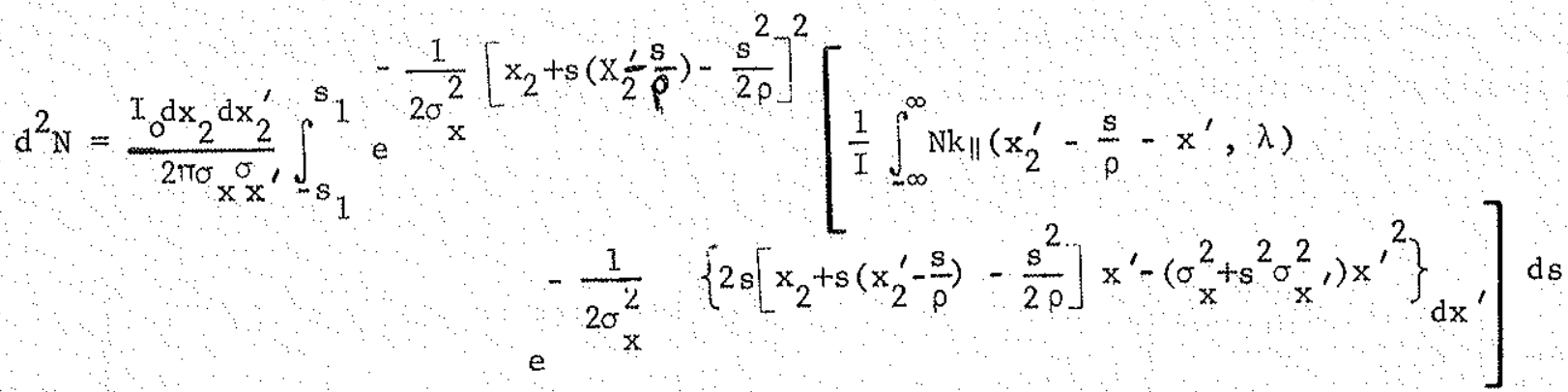

\section{Approximation of the Source}

The expressions of the preceeding sections can be systematized and numerically integrated, but they are too unwieldy for estimating the effects of the several parameters. The electron distribution is described by a norma 1 probability function and, since the manipulation of these functions is well known, approximation of the radiation distribution by a probability function is indicated. From (17)(19)(20)(21)

$$
\begin{aligned}
& N_{k}(\lambda)=1.256 \times 10^{16} \mathrm{k} \theta \mathrm{I} \gamma G_{1} \\
& N_{k \|}(\psi, \lambda)=3.461 \times 10^{15} \mathrm{k} \theta I \gamma^{2} \mathrm{~F}_{\|}(\psi) \text { per rad } \\
& N_{k \|}(0, \lambda)=3.461 \times 10^{15} \mathrm{k} \theta \mathrm{I} \gamma^{2} \mathrm{H}_{2}
\end{aligned}
$$


If we set $\quad N_{k \|}(\psi, \lambda)=g(\lambda) \times \frac{1}{\sqrt{2 \pi} \sigma} e^{-\frac{\psi^{2}}{2 \sigma^{2}}}$

then $N_{k} H(0, \lambda)=g(\lambda) \times \frac{1}{\sqrt{2 \pi} \sigma}$

and $\quad g(\lambda)=3.461 \times 10^{15} \mathrm{k} \theta \mathrm{I \gamma}^{2} \mathrm{H}_{2} \times \sqrt{2 \pi} \sigma$

But $\quad \int_{-\infty}^{\infty} N_{k} l(\psi, \lambda) d \psi=g(\lambda)=f N_{k}(\lambda)=1.256 \times 10^{16} k \theta I_{\gamma f G}$

where $f$ is the fraction of total radiation at $\lambda$ which is $\|$ polarized, and is plotted in Fig. 14.

From (50) (51)

$$
\gamma_{\sigma}=1.448 \frac{\mathrm{fG}_{1}}{\mathrm{H}_{2}} \quad(\mathrm{rad})
$$

and is a function only of $\lambda_{c} / \lambda$, plotled in Fig. 20. It is approximately

$$
\gamma \sigma=0.565\left(\lambda / \lambda_{c}\right)^{0.425}
$$

and $N_{k} \|(\psi, \lambda)=3.461 \times 10^{15} k \in I \gamma^{2} H_{2} e^{-\frac{\psi^{2}}{2 \sigma^{2}}}$ (per rad)

The exponential of (53) is compared in Fig. 21 with the Bessel function curves of Fig. 15. The agreement is good up to $\lambda / \lambda_{c}=10$ and is fair at 100.

If we again start at an electron beam minimum, the current in dydy' is given by

$$
d^{2} I=I_{0} P\left(y, y^{\prime}\right) d y d y^{\prime}
$$

and the one $\sigma$ contour of the beam at $s=0$ by (31). When transformed to $s$ the electron beam is described by (33) and (40). In the $y, y^{\prime}$ plane at $s=s$ the current in $d y d y^{\prime}$ is

$$
d^{2} I=I_{0} P_{1}\left(y, y^{\prime}\right) d y d y '
$$


with $P_{1}$ given by (41). Let the electrons radiate from arc $\theta=\mathrm{ds} / \rho$ and (53) becomes

$$
\begin{aligned}
& \mathrm{dN}_{\mathrm{k}} \|(\psi, \lambda)=\mathrm{AI} \mathrm{ds} \frac{1}{\sqrt{2 \pi} \sigma} \mathrm{e}^{-\frac{\psi^{2}}{2 \sigma^{2}} \mathrm{~d} \psi} \\
& \mathrm{A}=3.46 \times 10^{15} \mathrm{k} \frac{\gamma^{2} \sqrt{2 \pi} \sigma \mathrm{H}_{2}}{\rho}
\end{aligned}
$$

The radiation angle, referred to s, is $Y^{\prime}$ so $\psi=Y^{\prime}-y^{\prime}$. Substitute I from (55) in (56) to determine photon flux in dy' from element dy dy' ds

$$
\begin{aligned}
d^{4} N_{k}=\operatorname{AI} d s \frac{1}{(2 \pi)^{3 / 2} \sigma \sigma_{y^{\sigma} y^{\prime}}} & \exp \left[-\frac{1}{2\left(1-r_{1}^{2}\right)}\left(\frac{y^{2}}{\sigma_{y}}-\frac{2 r_{1} y^{\prime}}{\sigma_{y_{1} \sigma_{1}^{\prime}}}+\frac{y^{\prime 2}}{\sigma_{y}^{\prime}}\right)\right] x \\
& x \exp \left[-\frac{1}{2 \sigma^{2}}\left(Y^{\prime}-y^{\prime}\right)^{2}\right] d y d y^{\prime} d y^{\prime}
\end{aligned}
$$

and to find the photon flux in $\mathrm{dy} \mathrm{dY}^{\prime}$ integrate (58) over a11 $\mathrm{y}^{\prime}$

$$
\begin{aligned}
d^{3} N_{k}= & A I d s \frac{d y d_{0}^{\prime}}{(2 \pi)^{3 / 2} \sigma \sigma_{y^{\sigma} y^{\prime}}} \exp \left[-\frac{1}{2}\left(\frac{y^{2}}{\sigma_{y_{1}}^{2}\left(1-r_{1}^{2}\right)}+\frac{y^{2}}{\sigma^{2}}\right)\right] x \\
& x \int_{-\infty}^{\infty} \exp \left\{-\left[\left(\frac{1}{2\left(1-r_{1}^{2}\right) \sigma_{y_{1}^{\prime}}^{2}}+\frac{1}{2 \sigma^{2}}\right) y^{\prime 2}-\left(\frac{r_{1} y^{2}}{\left(1-r_{1}^{2}\right) \sigma_{y_{1} \sigma_{1}^{\prime}}}+\frac{y^{\prime}}{\sigma^{2}}\right) y^{\prime}\right]\right\} d y^{\prime}
\end{aligned}
$$

but $^{9} \quad \int_{-\infty}^{\infty} e^{-\left(a x^{2}+2 b x\right)} d x=\sqrt{\frac{\pi}{a}} e^{\frac{b^{2}}{a}}$ so (59) becomes

$$
\begin{aligned}
& -\frac{1}{2\left[\sigma^{2}+\sigma_{y_{1}^{\prime}}^{2}\left(1-r_{1}^{2}\right)\right]}\left[\frac{\sigma^{2}}{\sigma_{y}^{2}} y^{2}-\frac{2 r_{1}{ }_{y}^{\prime}}{\sigma_{y_{1}}} y_{1}^{\prime}+y^{\prime 2}\right] \\
& \sigma_{y^{\prime}}^{2}=\sigma_{y_{1}^{\prime}}^{2}+\sigma^{2}
\end{aligned}
$$

If the exponential is put in the format of (34) 


$$
-\frac{1}{2\left(1-x_{2}^{2}\right) \sigma_{y_{2}}^{2}}\left(\frac{y^{2}}{\sigma_{2} \sigma_{2}^{\prime}}+\frac{2 x_{2} y^{\prime}}{\sigma_{y_{2}^{\prime}}^{\prime}}\right)
$$

The coefficients are

$$
\begin{aligned}
& \sigma_{\mathrm{Y}}^{2}=\sigma_{\mathrm{y}}^{2}=\sigma_{1}^{2}+\sigma^{2}=\sigma_{\mathrm{y}}^{2}+\sigma^{2} \\
& \sigma_{y_{2}}^{2}=\sigma_{y_{1}}^{2}=\sigma_{y}^{2}+s^{2} \sigma^{2} \\
& \mathrm{r}_{2}^{2}=\mathrm{x}_{1}^{2} \sigma_{\mathrm{y}_{1}^{\prime}}^{2} /\left(\sigma_{y_{1}^{\prime}}^{2}+\sigma^{2}\right) \\
& \left(1-x_{2}^{2}\right)=\left[\sigma^{2}+\sigma_{y_{1}^{\prime}}^{2}\left(I-x_{1}^{2}\right)\right] /\left(\sigma_{y_{1}^{\prime}}^{2}+\sigma^{2}\right)
\end{aligned}
$$

This flux in dy dy' from ds must now be transformed back to $s=0$ by

$$
\left|\begin{array}{cc}
1 & -s \\
0 & 1
\end{array}\right|
$$

The exponential of $(60)$ then becomes

$$
\exp \left\{-\frac{1}{2\left(1-x_{2}^{2}\right)}\left[\frac{y^{2}}{\sigma_{y_{2}}^{2}}+\left(\frac{2 s}{\sigma_{y_{2}}^{2}}-\frac{2 x_{2}}{\sigma_{y_{2} y_{2} \sigma_{2}}}\right) y^{\prime}+\left(\frac{s^{2}}{\sigma_{y_{2}}^{2}}-\frac{2 x_{2} s}{\sigma_{y_{2} \sigma_{2}{ }^{\prime}}}+\frac{1}{\sigma_{y_{2}^{\prime}}}\right) y^{\prime 2}\right]\right\}
$$

and equating coefficients to the form of (34), plus (62)

$$
\begin{gathered}
\sigma_{\mathrm{y}}^{2}=s^{2} \sigma^{2}+\sigma_{\mathrm{y}}^{2} \\
\sigma_{\mathrm{y}_{3}^{\prime}}^{2}=\sigma_{\mathrm{y}_{2}^{\prime}}^{2}=\sigma_{\mathrm{y}^{\prime}}{ }^{2}+\sigma^{2}=\sigma_{\mathrm{Y}}{ }^{2}
\end{gathered}
$$




$$
\begin{aligned}
& \left(1-r_{3}^{2}\right)=\frac{\sigma^{2}{ }^{2} y_{3}{ }^{2}+\sigma^{2} \sigma^{2} y}{\sigma_{y}{ }^{2} \sigma_{Y}{ }^{2}}=\frac{\sigma_{y}^{2}{ }^{2}{ }^{2}+s^{2} \sigma^{2} \sigma_{y}{ }^{2}}{\sigma_{y_{3}}{ }^{2}{ }_{Y^{\prime}}{ }^{\prime}} \\
& -\frac{\sigma^{2}\left(\sigma_{y}^{2}+s^{2} \sigma^{2}\right)+\sigma^{2} \sigma_{y}^{2}}{\left(\sigma^{2}+s^{2} \sigma^{2}\right)\left(\sigma_{y}^{2}+\sigma^{2}\right)}
\end{aligned}
$$

then from (63) and (64), (60) transforms to

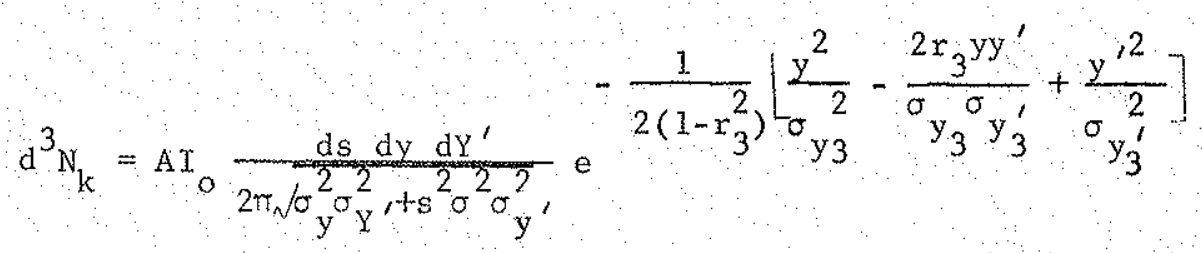

or substituting from (64)

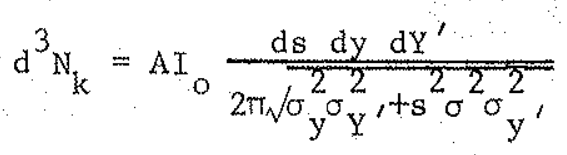

$$
\begin{aligned}
& -\frac{1}{2\left(\sigma_{y}^{2} \sigma_{y}^{2}+s^{2} \sigma^{2} \sigma_{y}^{2}\right.}\left[\left(\sigma_{y}^{2},+\sigma^{2}\right) y^{2}-2 s \sigma^{2} y x^{\prime}+\left(\sigma_{y}^{2}+s^{2} \sigma^{2}\right) y^{\prime 2}\right]
\end{aligned}
$$

This $\mathrm{d}^{3} \mathrm{~N}_{\mathrm{k}}$ is the number of photons in $d y \mathrm{dY}^{\prime}$ in the "source plane" $\mathrm{s}=0$, which come from radiation along $d s$ at $s=s$.
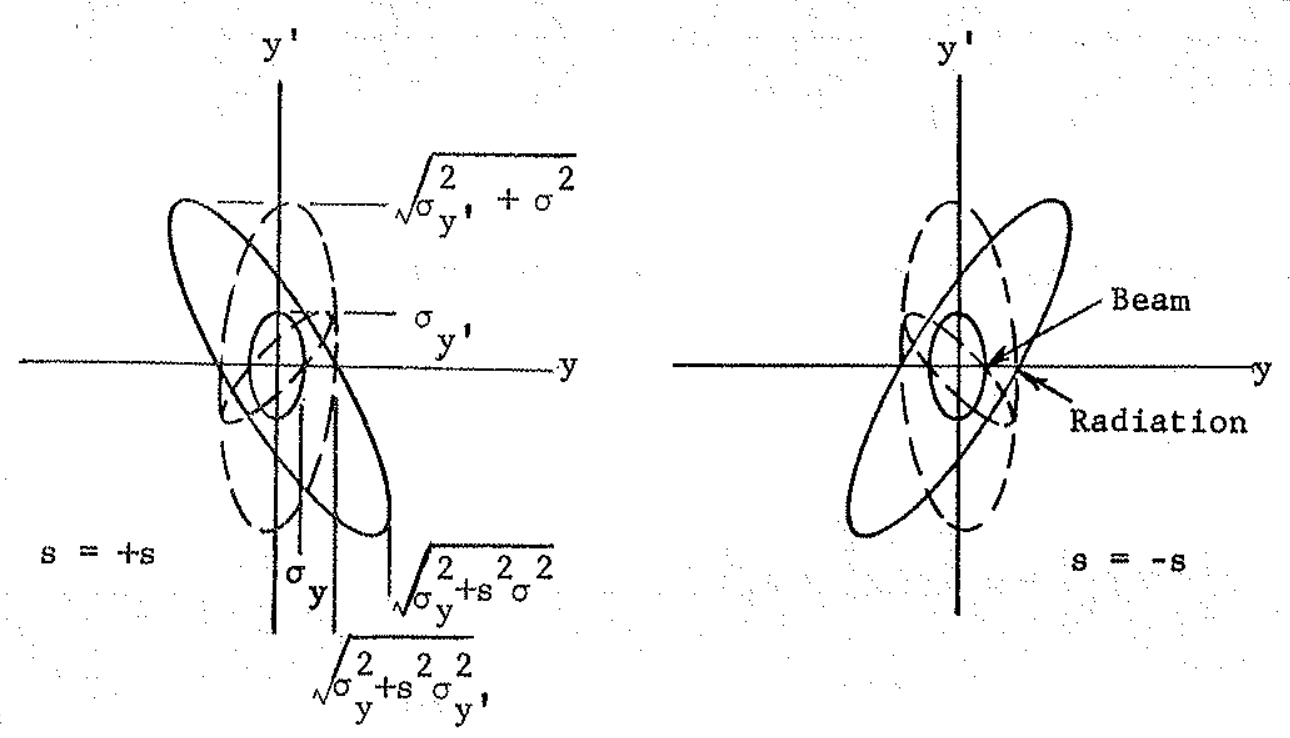

Fig. 22 Successive transmations in vertical $\left(y, y^{\prime}\right)$ phase space 
These successive transformations are shown graphically in Fig. 22 in which the example is exaggerated, as if from the end of a very long source. Most real sources, fortunately, have less rotation of the el1ipses. The contours are o (or no) ellipses. The electron beam ellipse at $s=0$ is upright, by arbitrary definition, It transforms, without magnetic focussing, to a slanted ellipse in which the angles $y^{\prime}$ are preserved but the size y increases. The radiation then increases the angles to form the real source and, at s, the density of this source will be less than at $s=0$. In order to synthesize the optical source the radiation is then transformed to $s=0$ with angles preserved but with increase in apparent size Once established, the radiation area in phase space is an invariant but its shape can be changed and distorted.

The radical in (65) is $\sigma_{y_{3}} \sigma_{3} \sqrt{1-r_{3}^{2}}$ and we can integrate over $y_{\text {, }}$ using the properties of bivariate distributions 9,10

$$
\begin{gathered}
d^{2} N_{k}=A I_{0} d s d y^{\prime} \frac{1}{\sqrt{2 \pi} \sigma_{y_{3}^{\prime}}} e^{-\frac{y^{\prime 2}}{2 \sigma^{2}} y_{3}^{\prime}}-\frac{1}{2} \frac{y^{\prime 2}}{\left(\sigma_{y}^{2},+\sigma^{2}\right)} \\
\quad=A I_{0} d s d y^{\prime} \frac{1}{\sqrt{2 \pi} \sqrt{\sigma_{y}^{2}+\sigma^{2}}}
\end{gathered}
$$

and since $s$ occurs only as ds this integrates over s as

$$
\mathrm{dN}_{k}\left(\mathrm{y}^{\prime}\right)=\int_{-s}^{s} \frac{\mathrm{d}^{2} \mathrm{~N}_{k}}{\mathrm{ds}} \mathrm{ds}=2 \mathrm{AI} \mathrm{I}_{0} \mathrm{dy} \cdot \frac{\mathrm{s}}{\sqrt{2 \pi} \sqrt{\sigma_{y}^{2},+\sigma^{2}}} \mathrm{e}^{-\frac{1}{2} \frac{y^{\prime 2}}{\left(\sigma_{y}^{2}+\sigma^{2}\right)}}
$$

Similarly integrate (65) over $y^{\prime}$ to obtain

$$
d^{2} N_{k}=A I \frac{d y}{\sqrt{2 \pi} \frac{d s}{\sigma_{y}^{2}+s^{2} \sigma^{2}}} e^{-\frac{1}{2}} \frac{y^{2}}{\left(\sigma^{2}+s^{2} \sigma^{2}\right)}
$$


Integration of (69) over s can be done by means of a function which I will call ef $(a, y)$, defined as

$$
e f(a, y)=\int_{0}^{y} \frac{1}{\cos t} e^{-\frac{a}{2} \cos ^{2} t} d t
$$

and normalized by

$$
\int_{-\infty}^{\infty} e f(a, Y) d a=\sqrt{2 \pi} \tan Y
$$

at $a=0 \quad$ ef $(0, Y)=\frac{1}{2} \ln \left(\frac{1+\sin Y}{1-\sin Y}\right)$

and for $Y<0.1 \quad$ ef $(a, Y) \simeq X e^{-\frac{a^{2}}{2}}$

Appendix $C$ has a description of the function together with a graph and short table of values.

If we set $\cos t=\sigma_{y} / \sqrt{\sigma_{y}^{2}+s^{2} \sigma^{2}}$ and $a=y / \sigma_{y}$, then 1imit $x$ is given by $\tan \mathrm{X}=\sigma s / \sigma_{\mathrm{y}}$

The integral of (69) over s then becomes (Appendix $\mathrm{C}$ )

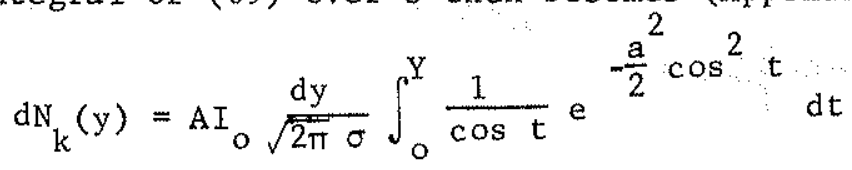

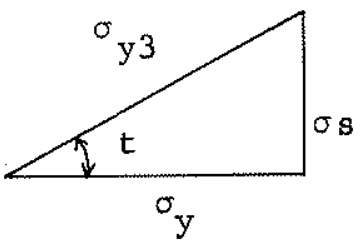

and the distribution of photons over $y$ in the plane $s=0$ is

$$
\mathrm{dN}_{\mathrm{k}}(\mathrm{y})=\mathrm{AI}_{\mathrm{o}} \frac{\mathrm{dy}}{\sqrt{2 \pi} \sigma} \text { ef }\left(\frac{\mathrm{y}}{\sigma_{\mathrm{y}}}, \tan ^{-1} \frac{\sigma \mathrm{s}}{\sigma_{\mathrm{y}}}\right)
$$

while the probability that a photon is in dy' at $y^{\prime}$ is, from (68)

$$
\mathrm{P}=\frac{\mathrm{dy}}{\sqrt{2 \pi} \sqrt{\sigma_{y}^{2}+\sigma^{2}}} e^{-\frac{1}{2} \frac{y^{\prime 2}}{\left(\sigma_{y}^{2}+\sigma^{2}\right)}}
$$

The distribution over $s=0$ with equal contributions from negative $s$ and positive $s$ is symmetrical about the $y$ axis, and is from (72) symmetrical about the y axis. There will be no cross terms of the independent variates of (71) and (72) and the equi-intensity contours will be symmetrical about the axes. We can then combine (71) and (72) to give the $y, y^{\prime}$ intensity in the synthesized source in the transverse plane $s=0$ with (71) $\times 2$ 


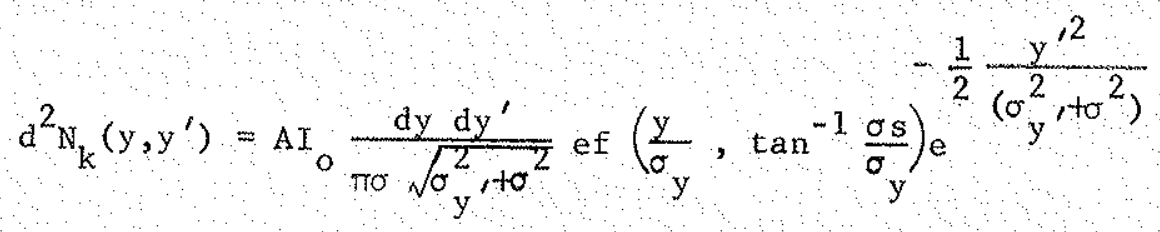

At $y=y^{\prime}=0(66)$ can be integrated from $-\mathrm{s}$ to $\mathrm{s}$ by ${ }^{11}$

$$
\begin{aligned}
& \int \frac{d x}{\sqrt{x^{2}+a^{2}}}=\sinh ^{-1} \frac{x}{a} \text { to give the central yy density } \\
& d^{2} N_{k}(0,0)=A_{0} \frac{d y d y^{\prime}}{\pi} \frac{1}{\sigma \sigma y^{\prime}} \sinh ^{-1}\left(\frac{s \sigma y^{\prime}}{\sigma \sigma^{\prime} y^{\prime}}\right)
\end{aligned}
$$

$I f A=\sqrt{2 \pi} \circ B$

$$
B=3.46 \times 10^{15} k \frac{y^{2}}{p} H_{2}
$$

and we can rewrite the y densities at $\mathrm{s}=0$;

$(71) \times 2$

$$
\begin{gathered}
\mathrm{dN}_{\mathrm{k}}(y)=2 B I_{0} d y \operatorname{ef}(a, Y) \\
a=\frac{y}{\sigma_{y}}, \tan y=\frac{\sigma s}{\sigma_{y}} \\
d N_{k}\left(y^{\prime}\right)=2 B I_{0} d y^{\prime} \frac{\sigma s}{\sqrt{\sigma_{y}^{2}+\sigma^{2}}} e^{-\frac{1}{2} \frac{y^{\prime 2}}{\left(\sigma_{y}^{2}, t^{2}\right)}}
\end{gathered}
$$

$$
e^{-\frac{1}{2} \frac{y^{2}}{\left(\sigma^{2}+\sigma^{2}\right)}}
$$

$$
d^{2} N_{k}(0,0)=\sqrt{\frac{2}{\pi}} B I \frac{d y d y^{\prime}}{\sigma y^{\prime}} \sinh ^{-1}\left(\frac{s \sigma \sigma y^{\prime}}{\sigma_{y^{\sigma} Y^{\prime}}}\right)
$$

In the $x, x^{\prime}$ plane the central orbit of the electron beam will project on the plane $s=0$ along the parabola (Fig. 19)

$$
x^{2}-2 x / \rho=0, s=\rho x^{\prime}
$$


Radiation from element ds at $s$ will transform to $s=0$ centered at $x^{\prime}=s / p$, $x=s^{2} / 2 p$. The one $\sigma$ contour of $x$ is, using (64), $\sigma_{x_{3}}^{2}=\sigma_{x}^{2}+s^{2} \sigma^{2}$ and since $s=p x^{\prime}$ we obtain the contour

$$
x=s^{2} / 2 \rho \pm \sqrt{\sigma^{2}+s^{2} \sigma^{2}}=\rho x^{2} / 2 \pm \sqrt{\sigma^{2}+\sigma^{2} \rho^{2} x^{2}}
$$

which is shown in Fig. 23.

The contour of (78) is an approximation based on the assumption that the contours have small curvature in the region considered. Since the radiation emitted by a single electron is paralle1 to the $x^{\prime}$ axis, a radiation distribution large compared to the curvature vs. $x$ ' would enlarge the contour.

The radiation is distributed uniformly along the arc so we can obtain the uniform $x$ density from (17) multiplied by fo obtain the number of || polarized photons;

$N_{k} \|(\lambda)=1.26 \times 10^{13} k I \gamma G_{1}$ f per sec,

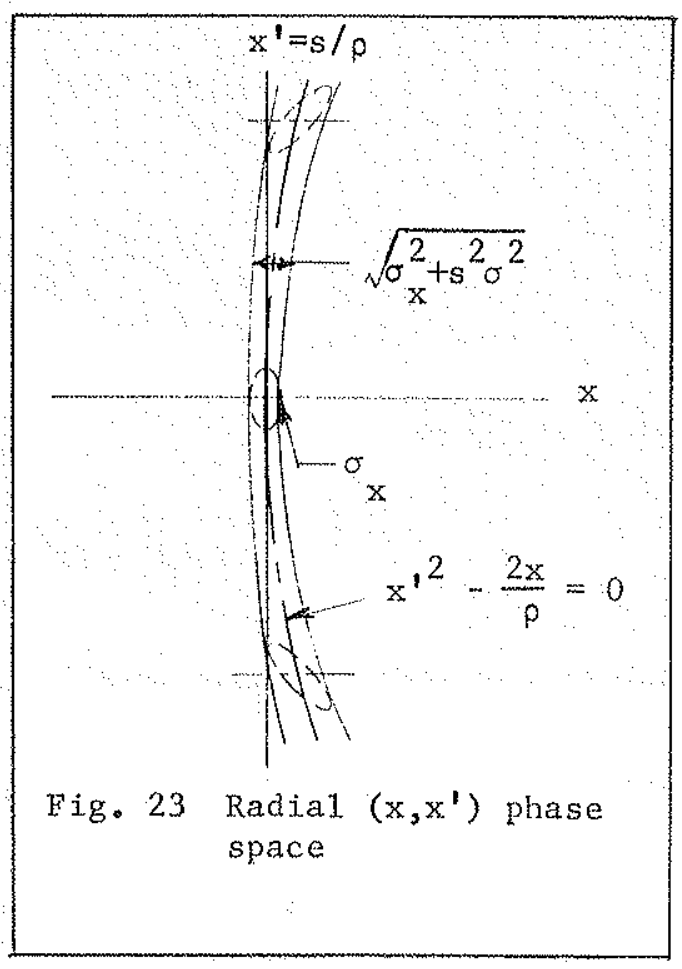
$\operatorname{mrad} \theta$

The probability that a photon from $s$ will be in $\mathrm{dx}$ is

$$
=e^{-\frac{1}{2} \frac{\left(x-s^{2} / 2 \rho\right)^{2}}{\left(\sigma_{x}^{2}+s^{2} \sigma^{2}\right)}}
$$

and the number of photons in $\mathrm{dx}_{\mathrm{dx}}$ is the product of (79) and (80)

times $d x^{\prime}$. At $x=x^{\prime}=0$ the central $x$ density is

$$
\mathrm{d}^{2} \mathrm{~N}_{\mathrm{k}}(0,0)=1.26 \times 10^{13} \mathrm{kI \gamma fG_{1 }} \frac{\mathrm{dx} \mathrm{dx}^{\prime}}{\sqrt{2 \pi} \sigma_{x}}
$$


Selection of the source center at a waist, or minimum, or focus of the electron beam made the derivation of the equations manageable. This type of source center is found at the center of a double focussing insertion in a ring and sometimes in the general lattice. However, the source center is often at a location $\bar{s}$ where the beam phase space ellipse is tilted and described by (28)

$$
\gamma y^{2}+2 \alpha y^{\prime}+\beta y^{2}=E
$$

The $\alpha, \beta, \gamma$ at the desired $s$ and $\mathrm{e}$ are obtained from the calculations of the ring lattice dynamics (See Appendix B). If the one o emittance is $\sigma_{y} \sigma y^{\prime}$ then, by comparing (28) and (33)

$$
\sigma_{y}=\sqrt{E \beta}, \sigma_{y}=\sqrt{E \gamma}
$$

The values at $s_{1}$, related to those at s are given by

$$
\begin{aligned}
& \beta_{1}=\beta-2 \alpha\left(s_{1}-\bar{s}\right)+\gamma\left(s_{1}-\vec{s}\right)^{2} \\
& \alpha_{1}=\alpha-\gamma\left(s_{1}-\bar{s}\right) \\
& \gamma_{1}=\gamma
\end{aligned}
$$

(provided there is not magnetic focussing). Alternatively (33) can be transformed by (32) with s replaced by $\left(s_{1}-\bar{s}\right)$. However, from (82)

$$
\alpha(s)=\alpha_{0}-\gamma(s) s
$$

measured from the location of $\alpha_{0}$. Set $\alpha_{0}=0$ at $s=0$ and the projected waist will be at

$$
\alpha(s)=-\gamma(s) s
$$

If we measure from this zero (33) and (34) need only to have s replaced by $s_{1}$, and similarly $(60)$ and $(62)$ have $s$ replaced by $s_{1}$. The transformation to the forms of $(63),(64)$ and (66) must be done with 


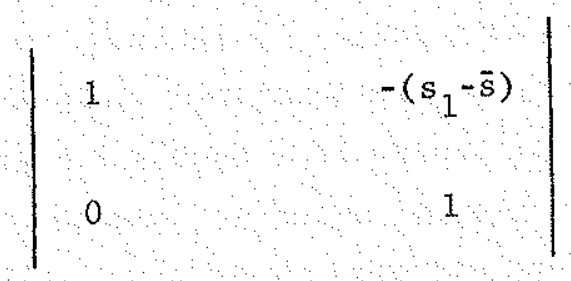

and the result is complex expressions and difficult integration which are beyond the scope of this paper. The symmetry assumption incorporated in (76) is no longer exact. The modification of the $x, x^{\prime}$ procedure is obvious, and is considerably simpler.

The values of the source parameters can be examined and if $\sigma y^{\prime}<\sigma$, so $<\sigma_{y}$ and $s \sigma_{y},<\sigma_{y}$ (preferably less than half) the approximations given above are reasonably good for a source not centered at a waist. For extreme cases the source length can be divided into a few $\Delta$ s, the flux calculated for each, and the results summed.

It is important to note that no al lowance has been made for spread of $x$ by momentum dispersion. Ring dynamics calculations provide a monentum compaction function $\mathrm{x}_{\mathrm{p}}$ defined by

$$
\Delta x=x_{p} \frac{\Delta p}{p}
$$

where $\Delta p$ is the semi-momentum spread of the beam. In an insertion the $x_{p}$ can be very small and need not be included. If $\Delta x$ is appreciable, compared to the betatron oscillation $\sigma_{x}$, then the $\sigma_{x}$ of $(80)$ and (81) is replaced by $\left(\sigma_{x}^{2}+\Delta x^{2}\right)^{\frac{2}{2}}$. The $x_{p}$ amplitude function usually does not vary rapidly with $\mathrm{s}$. As a result the modulation of angle $\mathrm{x}^{\prime}$ by $\mathrm{dx}_{\mathrm{p}} / \mathrm{d}$ s is small and has only a small effect on the assumption that the radiation is uniform with $\theta$.

In sum, the symchrotron light source is characterized by general parameters

$$
\gamma, p,\left(\lambda_{c} / \lambda\right), \tau_{0},
$$

by specific parameters

$$
\sigma_{\mathrm{x}}, \sigma_{\mathrm{y}}, \sigma_{\mathrm{x}}, \sigma_{\mathrm{y}}, \mathrm{s},
$$

and in some configurations also by

$$
\alpha, \beta, \gamma, x_{p}
$$




\section{Radiation Beam Power}

The power which can be encountered in a synchrotron radiation beam has an important influence on optical design. Cooling and thermal distortion of the first optical element may well. limit the flux which can be gathered from electron storage rings. It is always advantageous, with respect to beam power, to work near $\lambda_{\mathrm{c}}$. Figure 10 shows the percentage of power at wavelengths greater than $\lambda$ vs. $\lambda / \lambda_{c}$, and it will be noted that half the power is radiated below $\lambda_{\mathrm{c}^{\circ}}$

The total radiated powez is current times the volts/turn (Table I)

$$
P=88.5 \mathrm{~L}^{4} \mathrm{I} / \rho \mathrm{kw} / \mathrm{amp} \text { or } \mathrm{w} / \mathrm{ma}
$$

or multiplying by $10^{-3} / 2 \pi$

$$
\begin{aligned}
P & =0.01409 \mathrm{E}^{4} \theta \mathrm{I} / \mathrm{\rho} \quad \mathrm{w} / \mathrm{ma} \text { mrad } \\
& =9.61 \times 10^{-16} \mathrm{\gamma}^{4} \theta \mathrm{I} / \mathrm{\rho} \quad\|"\|
\end{aligned}
$$

is the total power intecepted from arc $\theta$.

By combining (84) and (16), $\mathrm{E}$ in GeV and $\lambda_{\mathrm{c}}$ in $\AA, P=0.0787 \mathrm{~L} / \lambda_{\mathrm{c}} \mathrm{w} / \mathrm{ma} \mathrm{mrad} \theta$

The power radiated as a function of the vertical angle $\psi$ is, from (9) (24)

$$
\begin{aligned}
P & =1.44 \times 10^{-18} \frac{y^{5}}{\rho} \mathrm{F}(\gamma \psi) \quad w / \operatorname{ma} \text { mrad } \theta, \psi \\
& 0.0413 \mathrm{E}^{5} \mathrm{~F}(\gamma, \psi) \quad \text { " }
\end{aligned}
$$

Function $F(\gamma \psi)$ is shown in $\mathrm{Fig} .12$ as we 11 as $\mathrm{F}_{7}(\gamma \psi)$ defined by $(24)$. $F(\gamma \psi)$ can be approximated closely by a norma 1 distribution

$$
\mathrm{F}(\gamma \psi)=\frac{k}{\sqrt{2 \pi} \sigma} e^{-\frac{1}{2} \frac{(\gamma \psi)^{2}}{\sigma^{2}}}
$$

which can be normalized by the total power from (84)

$$
\int_{-\infty}^{\infty} F(\gamma \psi) d(\gamma \psi)=\gamma \int_{-\infty}^{\infty} F(\gamma \psi) d \psi=k \quad(\gamma \psi \text { in rad })
$$

and since the integral of (86) is $k$, 
so $\quad \int P d \psi=1.44 \times 10^{-15} \frac{\gamma^{4} k}{\rho}=9.61 \times 10^{-16} \frac{\gamma^{4}}{\rho} ; \quad k=0.667$

and at 0

$$
\begin{aligned}
& F(\gamma \psi)=\frac{k}{\sqrt{2 \pi} \sigma}=0.4375 \\
& \sigma_{\psi}=0.608 \text { rad of } \gamma \psi \\
& F(\gamma \psi)=0.4375 \mathrm{e}^{-\frac{1}{2}} \frac{(\gamma \psi)^{2}}{(0.608)^{2}} \quad(\psi \text { in rad })
\end{aligned}
$$

It is often convenient to represent the power distribution by a rectangle of "width" $\psi=2 / \gamma$. If in (85)

$$
\begin{gathered}
\int_{0}^{\infty} F(\gamma \psi) d \psi=k_{1} / \gamma \\
2 \times 1.44 \times 10^{-15} \frac{\gamma^{4}}{\rho} k_{1}=9.61 \times 10^{-16} \frac{\gamma^{4}}{\rho} \\
k_{1}=0.334 \mathrm{rad} \text { of } \gamma \psi
\end{gathered}
$$

The rectangle is shown in Fig. 12. If this approximation is used it is sometimes necessary to remember that the peak power is larger in the ratio 1.31 。

If we assume the beam is very small there is an approximation useful for estimating. At one meter from the source one mrad $\theta$ subtends $10^{-3} \mathrm{~m}$ and $\psi=2 / \gamma$ subtends $2 / \gamma \mathrm{m}$ so the radiation falls on $2 \times 10^{-3} / \mathrm{\gamma} \mathrm{m}^{2}$. But the power from one mrad $\theta$ is (84)

$$
P=9.61 \times 10^{-16} \gamma^{4} / \rho \quad w / m a \operatorname{mrad} \theta
$$

and the specific power

$$
\mathrm{P} / \mathrm{A}=4.80 \times 10^{-13} \gamma^{5} / \rho \mathrm{w} / \mathrm{ma} \mathrm{m}^{2} \text { at } 1 \mathrm{~m}
$$

If $D$ is the distance from the source in $m$

$$
\mathrm{P} / \mathrm{A}=1.38 \mathrm{E}^{5} / \rho \mathrm{D}^{2} \mathrm{w} / \mathrm{ma} \mathrm{cm}^{2}
$$


If the angular spread of the electron beam is appreciable this power will. be reduced by the approximate ratio (see (93))

$$
\gamma^{-1} / \sqrt{\gamma^{-2}+\alpha^{2}}
$$

where $\alpha$ is the angular divergence of the beam.

The preceeding relations apply to an electron beam which is a filament. Distribution of the beam in $y$ and $y^{\prime}$ will reduce the power density, often by a significant factor. In the vertical coordinates the electron beam is described (55) by

$$
d^{2} I=\frac{I_{0}}{2 \pi \sigma_{y} y^{\prime}} e^{-\frac{1}{2}\left(\frac{y^{2}}{\sigma_{y}^{2}}+\frac{y^{\prime 2}}{\sigma_{y^{\prime}}}\right)} d y d y^{\prime}
$$

and the current in element dy dy' wi.l radiate power in angle element $d \psi$ at $\psi$, using (85) in radians of $\psi$ and (87)

$$
\mathrm{d}^{3} \mathrm{P}=\mathrm{d}^{2} \mathrm{I}\left(1.44 \times 10^{-15} \frac{x^{5}}{\rho}\right) \frac{k_{2}}{\sqrt{2 \pi \sigma_{\psi}}} \mathrm{e}^{-\frac{1}{2}-\frac{\psi^{2}}{\sigma^{2}}} \mathrm{~d} \psi \text { per mrad } \theta \text {, ma, rad } \psi
$$

at $\psi=0$

$$
\mathrm{k}_{2} / \sqrt{2 \pi} \sigma_{\psi}=0.4375 \text { and } \sigma_{\psi}=0.608 / \gamma
$$

so $\quad d^{3} P=d^{2} x\left(9.61 \times 10^{-1.6} \frac{y^{4}}{\rho}\right) \frac{1}{\sqrt{2 \pi} \sigma_{\psi}} e^{2} \frac{\psi^{2}}{\sigma^{2}}$

$$
-\frac{1}{2} \frac{\psi^{2}}{\sigma_{\psi}^{2}}
$$

but if the radiation angle is $Y^{\prime}, \psi=Y^{\prime}-y^{\prime}$, and the power in element dy $d y^{\prime}$ radiated from current in dy dy' is

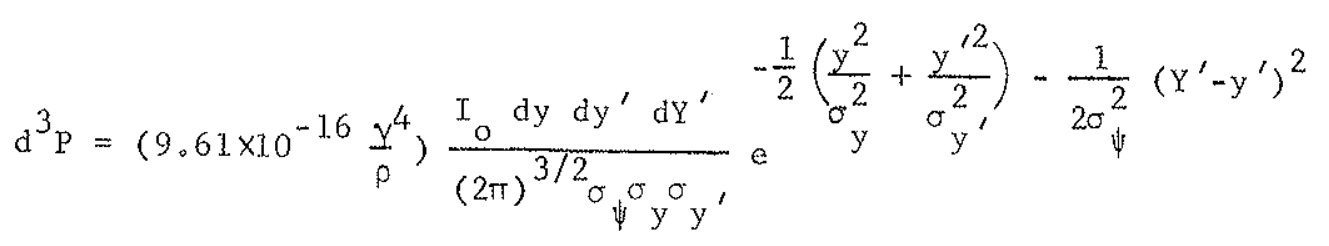

If we integrate over all $y^{\prime}$ the power radiated from element $d y d y$ ' of the source is (assuming one mrad $\theta$ and $I_{0}$ in ma)

$$
d^{2} p=\left(9.61 \times 10^{-16} \frac{y^{4}}{\rho}\right) \frac{I_{0} d y d Y^{\prime}}{2 \pi \sigma_{y \sigma} \sigma} e^{-\frac{1}{2}\left(\frac{y^{2}}{2}+\frac{y^{\prime 2}}{\sigma_{0}^{2}}\right)}
$$


and $\quad \sigma_{0}^{2}=\sigma_{y}^{2}+\sigma_{\psi}^{2}$

$\sigma_{\psi}=0.608 / \gamma \mathrm{rad}$

If the beam height $y$ is sma11, integrate over y to obtain the angular distri.bution

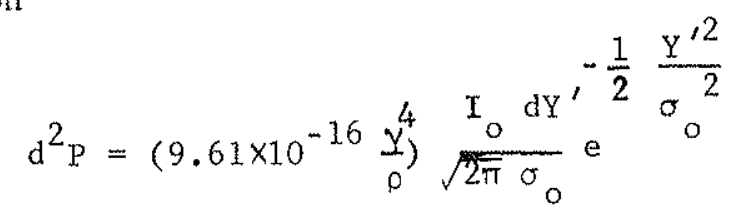

Units of (94) and (95) are watts per mrad $\theta$ per ma.

Equations (95) and (96) describe the source. The power density can be transformed to surfaces by using the methods of sect. II2. 
IV. Optical Transformations

The characteristics of sources can be most readily examined with linear paraxial transformations. These transformations map the elements of phase space and represent the best conditions that can be achieved. Aberrations and losses are beyond the scope of this section, but source properties and comparisons can be examined with idealized assumptions.

1. Non-Focusing Optics

The optical axis, $s$, is the central ray of the system. Deviations from $s$ are, in the horizontal and vertical planes, specified by $x, x^{\prime}$ and $y, y^{\prime}$. If there is no refraction or change of momentum the $s$ phase space ( and time) are preserved and can be ignored." The four dimensional phase space $x, x^{\prime}, y, y^{\prime \prime}$ is invariant but, if there is no $x y$ coupling, the $x, x^{\prime}$ and $y, y^{\prime}$ phase spaces are independently invariant.

A ray at $y_{0}, y_{0}^{\prime}$ propagating through free space from $D=0$ will be at $D=D_{1}$, at

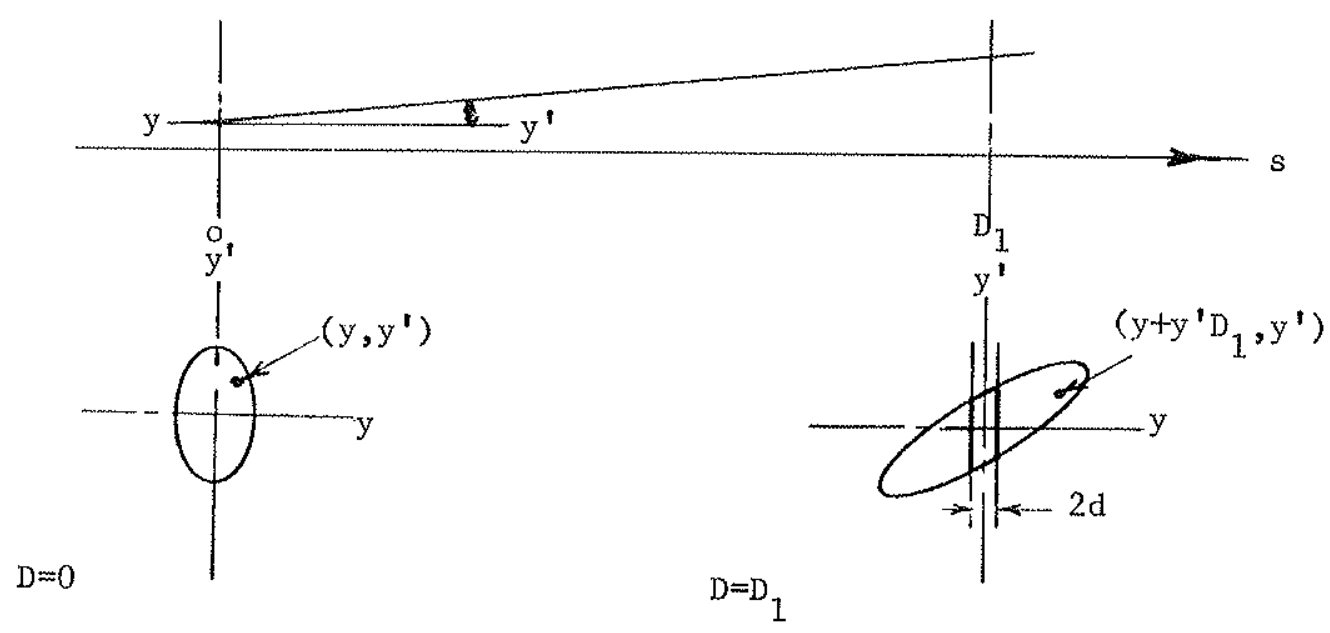

Fig. 24 Slit in vertical phase space

$$
y=y_{0}+y^{\prime} D_{1}, y^{\prime}=y_{0}^{\prime}
$$

and the free space transformation is

$$
\left|\begin{array}{l}
y \\
y^{\prime}
\end{array}\right|=\left|\begin{array}{ll}
1 & D_{1} \\
0 & 1
\end{array}\right|\left|\begin{array}{l}
y_{0} \\
y_{0}^{\prime}
\end{array}\right|
$$

* The effects of $s$ are folded into $x$ and $y$. 
Suppose a source at $D=0($ Fig. 24) with contour

$$
\frac{\Sigma_{y^{\prime}}}{\Sigma_{y}} y^{2}+\frac{\Sigma_{y}}{\Sigma_{y^{\prime}}} y^{\prime 2}=\Sigma_{y} \Sigma_{y^{\prime}}
$$

To avoid utter chaos in nomenclature, $\Sigma$ will be used as the variance, or equivalent variance, of the synthesized optical source; $\sigma$ will be reserved for the variance of the electron beam. The emittance of this source is $\Sigma_{y} \Sigma_{y^{\prime}}$ and the phase space area is $\pi \Sigma_{y} \Sigma_{y^{\prime}}$. Maxima are the axial intercepts $\hat{y}=\Sigma_{y}, \hat{y}^{\prime}=\Sigma_{y^{\prime}}$. If this source is transformed by (96) to $\mathrm{D}_{1}$ the contour will be

$$
\frac{\Sigma_{y^{\prime}}}{\Sigma_{y}} y^{2}-\frac{2 \Sigma_{y^{\prime}} D}{\Sigma_{y}} y y^{\prime}+\left(\frac{\Sigma_{y}}{\Sigma_{y^{\prime}}}+\frac{\Sigma_{y^{\prime}} D^{2}}{\Sigma_{y}}\right) y^{\prime 2}=\Sigma_{y} \Sigma_{y^{\prime}}
$$

Area and emittance are preserved but the maxima axe no longer the intercepts. They are (Fig. 18)

$$
\hat{y}=\sqrt{\Sigma_{y}^{2}+\Sigma_{y^{\prime}}^{2} D^{2}}, \hat{y}^{\prime}=\Sigma_{y^{\prime}}
$$

and this shows, as expected, that the angular distribution is unaltered but the size increases.

One of the most common, and important, optical instruments is the slit. It will be considered here in vertical dispersion. Suppose a slit of width $2 d$ at $D_{1}$ (Fig. 24). We can examine the properties by transforming the slit back onto the source or by transforming the source onto the slit. The latter seems preferable. The intercept of the source contour on $y^{\prime}$ is

$$
y=0, y^{\prime}=\frac{\Sigma_{y} \Sigma_{y^{\prime}}}{\sqrt{\Sigma_{y}^{2}+\Sigma_{y^{\prime}}^{2} D^{2}}}
$$

and the minimum angular resolution (semi-angle) as the slit width goes to 0 is $\Sigma_{y} \Sigma_{y} / \sqrt{\Sigma_{y}^{2}+\Sigma_{y^{\prime}}^{2} D^{2}}$. Slope of the contour at $y=0$ is, by differentiating ( 97 ) 


$$
\frac{d^{\prime}}{d y}=\frac{D}{\left(\Sigma_{y} / \Sigma_{y^{\prime}} j^{2}+D^{2}\right.}
$$

and the maximum angle through the slit is approximately

$$
\frac{\Sigma \Sigma_{y} y^{\prime}}{\sqrt{\Sigma_{y}^{2}+\Sigma_{y}^{2} D^{2}}}+\frac{D d}{\left(\Sigma_{y} / \Sigma_{y^{\prime}}\right)^{2}+D^{2}}
$$

The area outlined in Fig. 24 is, in most practical situations, nearly a parallelogram of area

$$
\frac{4 d \sum y^{\prime} y^{\prime}}{\sqrt{\Sigma_{y}^{2}+\Sigma_{y}^{2} D^{2}}}
$$
and since the emittance area is $\pi \sum_{y} \sum^{\prime}$ the fraction of flux passed
by the slit is

$$
\frac{4 d}{\pi \sqrt{\Sigma_{y}^{2}+\Sigma_{y^{\prime}}^{2} D^{2}}}
$$

Eq. 103 assumes uniform density, which may be a poor approximation. A somewhat better approximation is obtained by the ratio of slit width to emittance width

$$
\frac{d}{\sqrt{\Sigma_{y}^{2}+\Sigma_{y^{\prime}}^{2} D^{2}}}
$$

It is sometimes desirable to integrate (76) over 2 d. 
In the radial or $x, x^{\prime}$ space the radiation beam will be distributed
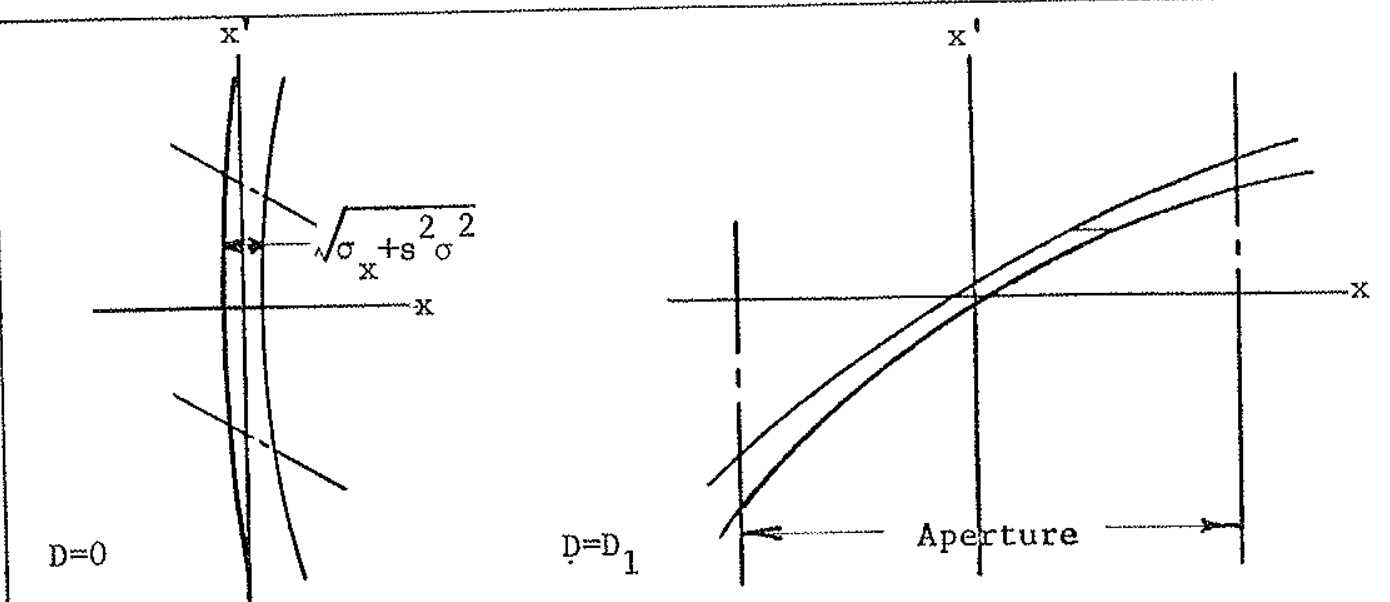

Fig. 25 Slit in radial phase space

along the length of the slit and will be cut off by the ends of the slit or by the aperture of the instrument illuminated. There will be a systematic variation of $x^{\prime}$, the horizontal angle, along the slit. The $x x^{\prime}$ density (Fig. 25) will vary as $\sqrt{\sigma_{x}^{2}+s^{2} \sigma^{2}}$, transformed to $\mathrm{D}=\mathrm{D}_{1}$ ( (see (78) and Fig. 23). In the vertical direction the angular distribution is given by $\mathrm{dN}_{k}\left(\mathrm{y}^{\prime}\right)$ of (76) and, if the sitt width $2 \mathrm{~d} \ll \hat{y}$ of (98) this distribution will be uniform across the width of the slit. However, the $\mathrm{dN}_{k}(y)$ of (76) has been integrated over $s$ and represents the sum of the source. Since the $y y^{\prime}$ density is correlated with the $x^{\prime}$ density through $s$, the y distribution given by (69) will vary over the length of the slit. For relatively short sources and moderate values of the various $\sigma$ the variations are sma 11 and in many, if not most, synchrotron light applications the slit is rather uniformly illuminated. Extreme cases can be analyzed by dividing the source length $s$ into a few $\Delta s$ and transforming the parts piecewise in order to maintain the correlation between $x$ and $y$. 
One important application of the slit occurs with the double crystal monochromator in the paralle1 position, now usually a channel cut crystal. The angular distribution passing the slit determines the resolution (if the angles are greater than the crystal angular window) and the resolution is limited by (100) or perhaps (101). With the extended source the resolution $\Delta \lambda / \lambda=\cot \theta \Delta y^{\prime}$ (Bragg angle $\theta$ ) is limited by the characteristics of the source and the distance to the slit, but not by the slit width. The distance to the slit and crystal are determined by the geometry. Flux is collected from orbit arc approximately $\theta=x / D$, where $x$ is the usable face width of the crysta1. Flux at the shorter x-ray wavelengths will of ten be 1 imited by the power the first elements can tolerate.

The double crystal spectrometer in the anti-parallel position is unique in that it is an angular slit in phase space.
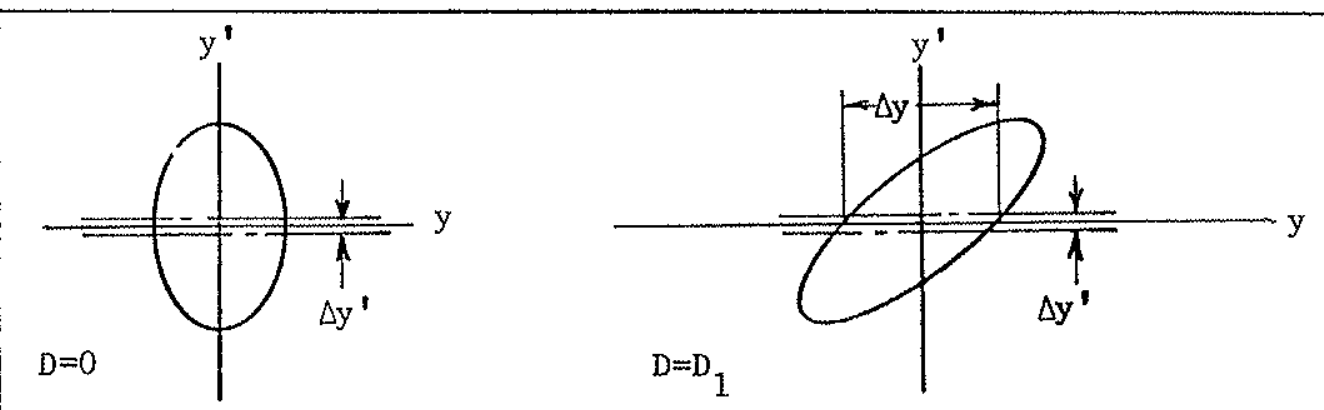

Fig. 26 Double crystal monochromator angular slit

Assuming vertical dispersion, the acceptance at the crystals is $\Delta y^{\prime}$ (Fig. 26) and this transforms back to the source as $\Delta y^{\prime}$. Angular acceptance is very sma11, with good crystals only a few seconds, so $\Delta y$ is nearly the same at $D=0$ and $D_{1}$. Usable $\Delta y$ is determined by the projected vertical height of the crystals and this will set one limit to source length $s$ by (73) or (76). The other limit is given by the width $w$ of the crystals which limits the source arc to $\theta=w / D=s / 0$ The smaller of these limits applies. However, the diffraction angle 
to the crystal planes is changed by the off axis angle $x^{\prime}$ in the amount $\frac{1}{2} x^{\prime 2} \tan \theta$ (this $\theta$ is the Bragg angle) which must be less than the crystal acceptance $\Delta y^{\prime}$. For this application the source angle $\theta / 2=x^{\prime}$ will seldom be limiting since it can be a few mradians for good crystals at Bragg angle of say $20^{\circ} . \Delta y^{\prime}$ is very small so the exponential in $\mathrm{dN}_{k}\left(\mathrm{y}^{\prime}\right)$ of $(76)$ is approximately one and

$$
\mathrm{dN}_{\mathrm{k}}\left(\mathrm{y}^{\prime}\right) \approx 7 \times 10^{15} \mathrm{kI}_{\mathrm{o}} \mathrm{\gamma}^{2} \mathrm{H}_{2} \theta \frac{\sigma}{\sqrt{\sigma_{\mathrm{y}^{\prime}}^{2}+\sigma^{2}}} \Delta \mathrm{y}^{\prime}
$$

with $\theta$ determined by the size of the crystals and the distance $D_{1}$. Diffraction studies such as those using Laue patterns or topography depend critically upon source size and source brightness. A specific study will require a specified angular spread across the sample. The situation is shown in Fig. 27 for two typical synchrotron light sources which can be used for $x$-ray diffraction. The slit may be either a pinhole or the edges of the crystal. If we rewrite (99)

$$
y^{\prime}=\frac{\Sigma_{y}}{\sqrt{\left(\Sigma_{y} / \Sigma_{y^{\prime}}\right)^{2}+D^{2}}}
$$
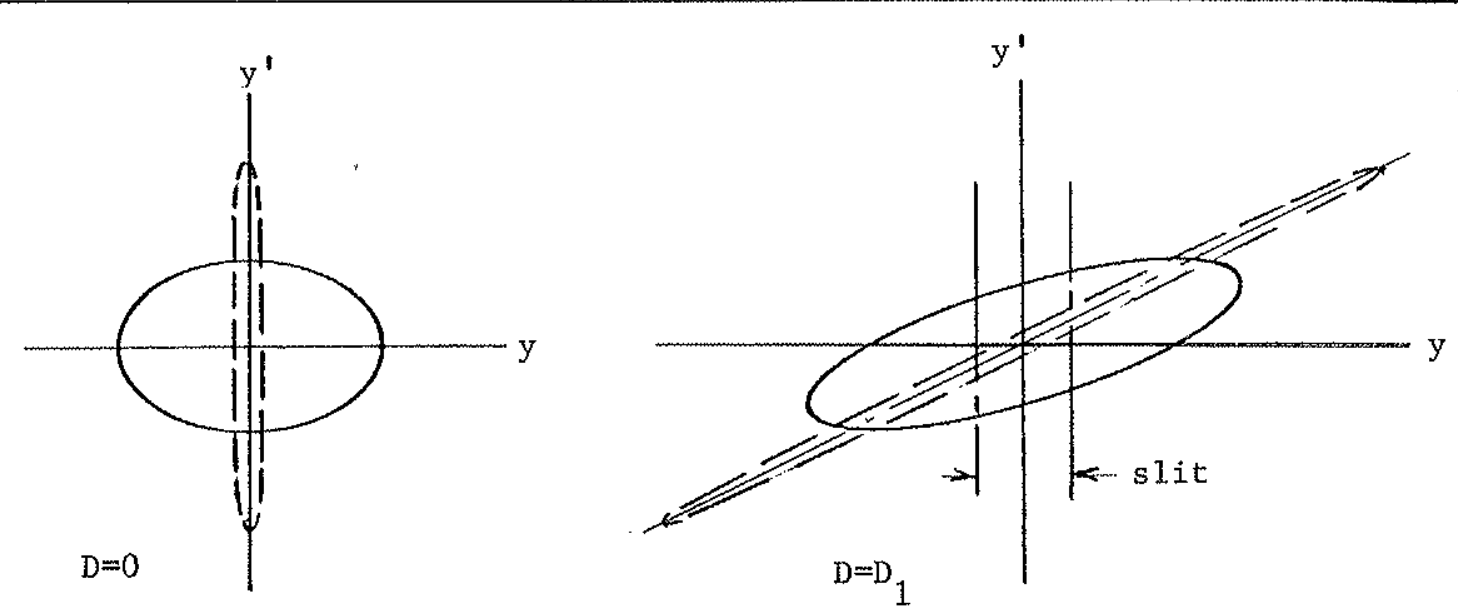

Fig. 27 Two typical sources, from different emittance beams, transformed to s1it in $y, y^{\prime}$ space 
The maximum semi-angle is roughly $\Sigma_{y} / D$, but $\Sigma_{y}$ for short sources is largely determined by $\sigma_{y}$. A similar situation obtains in $x^{\prime}$ (see Fig. 25). The $\sigma_{x}$ and $\sigma_{y}$ of various synchrotron light sources will take values in a range of more than an order of magnitude, and this can force a corresponding range of $D_{1}$. (One must of course retreat far enough to il luminate most of the sample and to prevent thermal decomposition of the materia1).

The flux passed through the small aperture at $\mathrm{D}_{1}$, and the exposure of the detector, depend upon the central brightness of the source. The central density functions in $x, x^{\prime}$ and $y, y^{\prime}$ are given by (81) and (76). These cannot simply be multiplied because we would then be counting the same photons twice. By the analogy of (65) or (66) to (76), the probability that a photon will lie in element dydy (at $y=y^{\prime}=0$ ) of the source is, from $(74)^{*}$

$$
p=\frac{d y d y}{\pi} \frac{1}{\sigma \sigma_{y^{\prime}}} \sinh ^{-1}\left(\frac{s \sigma \sigma_{y^{\prime}}}{\sigma_{y} \sigma_{Y^{\prime}}}\right)
$$

and this times ( 81 ) gives the central brightness

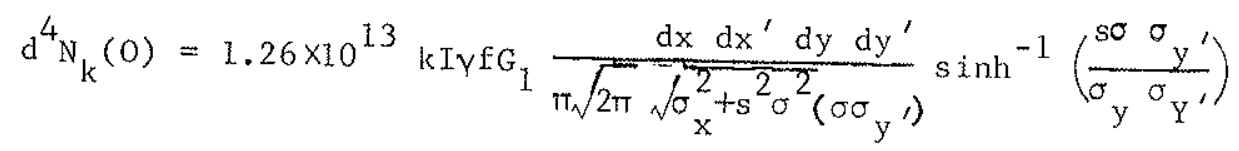

For diffraction sources the argument of $\sinh ^{-1}$ is less than 1 and $\sinh ^{-1} \mathrm{x} \approx \mathrm{x} ; \mathrm{s} \sigma$ tends to be small compared to $\sigma_{\mathrm{x}} \cdot \mathrm{Eq}$. (106) can
then be approximated as:

$$
d^{4} N_{k}=k_{2} \gamma\left(\frac{s}{\left(\sigma_{x} \sigma_{y} \sigma^{\prime}\right.}\right) \Delta x \Delta x^{\prime} \Delta y \Delta y^{\prime}
$$

with $k_{2}$ a numerical constant involving $k, I_{0}$ and functions of $\left(\lambda_{c} / \lambda\right)$. The phase space area subtended by the diffraction sample will almost a lways be small so $\mathrm{dx}$. . . can be replaced by $\Delta x$. . . and we can avoid integrating. Good $\mathrm{x}$-ray sources will have $\sigma_{\mathrm{Y}^{\prime}} \approx \sigma$ and this varies as $\gamma^{-1}$ at a fixed $\left(\lambda_{c} / \lambda\right)$. The brightness is thus proportional to $\gamma^{2}$ and inversely to $\sigma_{x} \sigma_{y}$, the electron beam size. Although source length $s$ is in the numerator, its magnitude is limited by the sample size and distance to $s=w_{p} / D$. Increasing $s$ and $\rho$ together has no influence on flux, which is proportional to $\theta=s / p$ and such increase will

Constant A of (74) contains a $o$ 
enlarge $\Sigma_{x}$ and $\Sigma_{y}$.

2. Focusing Optics

An optical system can be reduced, in first order, to a focal length $f$ and the location of two principal planes.

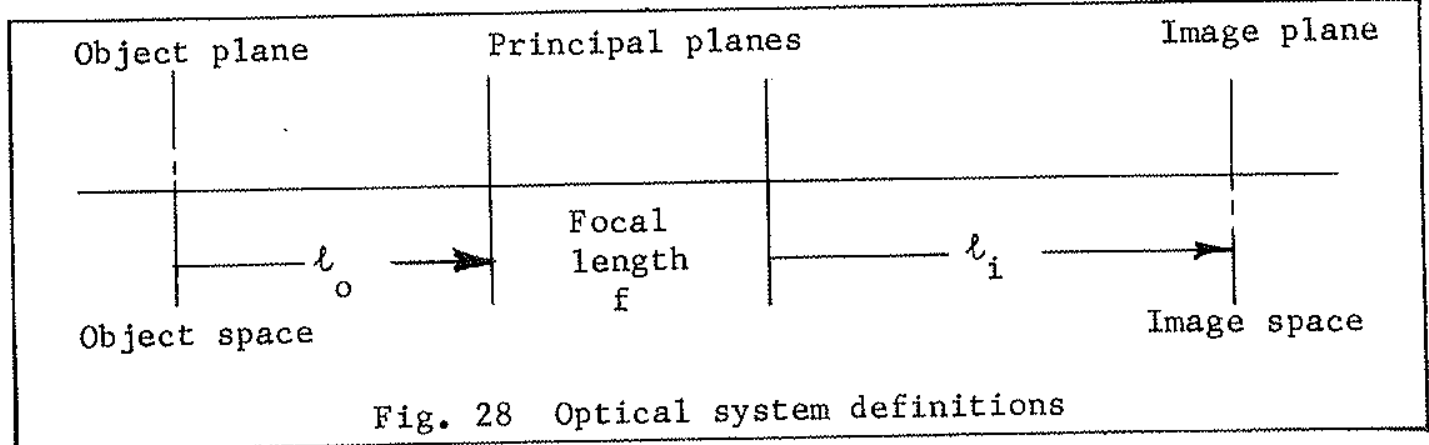

The transformation from the first to the second principal plane is

$$
\left|\begin{array}{rr}
1 & 0 \\
-\frac{1}{5} & 1
\end{array}\right|
$$

and to transform from $y_{0}, y_{0}^{\prime}$ in the object space to $y_{i}, y_{i}^{\prime}$ in the image space

$$
\left|\begin{array}{c}
y_{i} \\
y_{i}^{\prime}
\end{array}\right|=\left|\begin{array}{cc}
1 & e_{i} \\
0 & 1
\end{array}\right|\left|\begin{array}{cc}
1 & 0 \\
-\frac{1}{f} & 1
\end{array}\right|\left|\begin{array}{cc}
1 & e_{0} \\
0 & 1
\end{array}\right|\left|\begin{array}{c}
y_{0} \\
y_{0}^{\prime}
\end{array}\right|
$$

or

$$
\begin{gathered}
\left|\begin{array}{c}
y_{i} \\
y_{i}^{\prime}
\end{array}\right|=\left|\begin{array}{cc}
1-\frac{l_{i}}{f} & \left(l_{0}-\frac{l_{i} l_{0}}{f}+l_{0}\right) \\
-\frac{1}{f} & \left(-\frac{l_{0}}{f}+1\right)
\end{array}\right|\left|\begin{array}{c}
y_{0} \\
y_{0}^{\prime}
\end{array}\right| \\
y=\left(1-\frac{l_{i}}{f}\right) y_{0}+\left(l_{0}-\frac{l_{i} l_{0}}{f}+l_{i}\right) y_{0}^{\prime}
\end{gathered}
$$


and for image formation, e.g. conjugate planes in the object and image spaces

$$
\ell_{0}-\frac{l_{i} l_{0}}{f}+l_{i}=0 \text { or } \frac{1}{l_{0}}+\frac{1}{l_{i}}=\frac{1}{f}
$$

also

$$
l_{0}=-l_{i} \frac{f}{f-l_{i}}, l_{i}=l_{0} \frac{f}{f-t_{0}}
$$

and linear magnification $M=\frac{f-l_{i}}{f}=\frac{f}{f-l_{0}}=\frac{l_{i}}{l_{0}}=\frac{y_{i}}{y_{0}}$

angular magnification is $1 / M$ and the transformation between conjugate planes is

$$
\left|\begin{array}{c}
y_{i} \\
y_{i}^{\prime}
\end{array}\right|=\left|\begin{array}{cc}
M & 0 \\
-\frac{1}{f} & \frac{1}{M}
\end{array}\right|\left|\begin{array}{c}
y_{0} \\
y_{0}^{\prime}
\end{array}\right|
$$

(Matrix optics ${ }^{12,13}$ are usually formulated with historical sign and coefficient conventions. I find the above formulation in which distance proceeds from left to right and the column vectors have position on top to be more convenient.)

If a source is focused on a $y$ slit the contours transform as in Fig. 29, which shows magnification arbitrarily set at $1 \frac{3}{2}$.

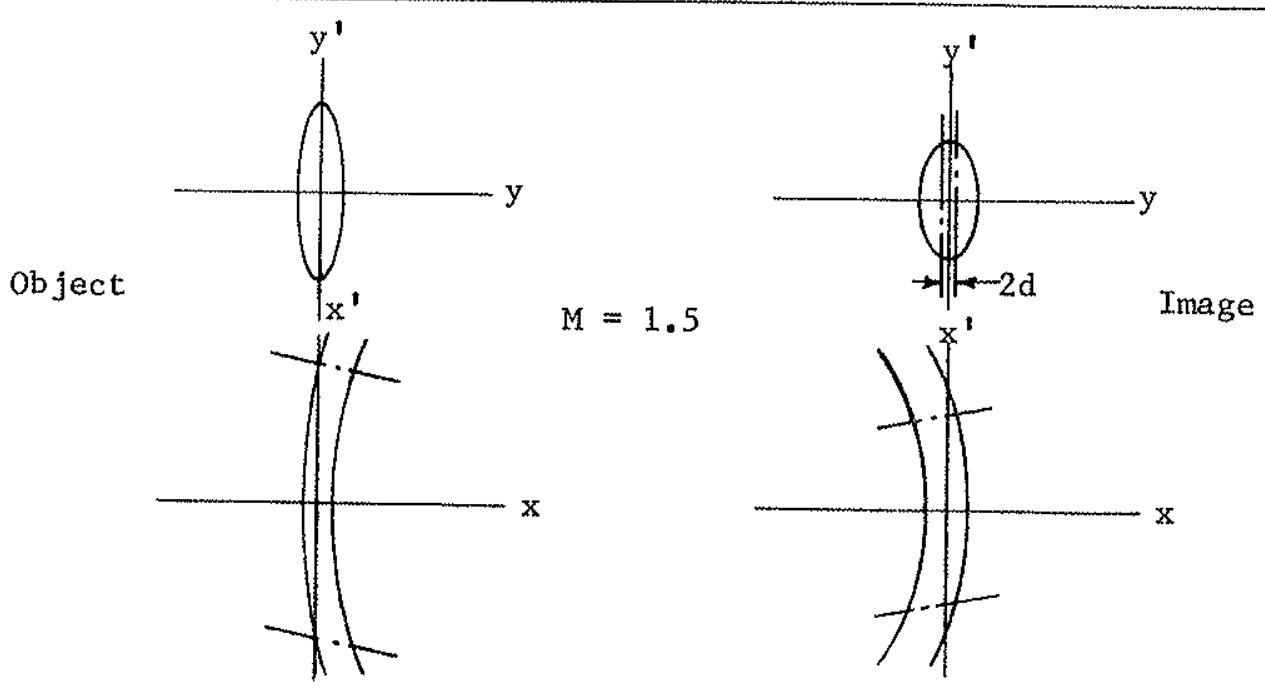

Fig. 29 Transformation by focussing system 
The $x, x^{\prime}$ figure is transformed to the system aperture stop (Fig. 25) and the stop then transformed back to the dashed line in Fig. 29. This determines $\theta$ and $s$, and the resulting source transforms to the image as shown in Fig. 29. The vertical, or $y$, source transforms as shown in Fig. 29 and it is assumed for this example that a $11 \mathrm{y}^{\prime}$ are passed by the system. (See Refs. 12, 13 for transformation of aperture stops.) Since the radial aperture has determined $s$ the flux passing the slit is from (76)

$$
N_{k}=\int_{-d}^{d} 2 B I_{o} \text { ef }\left(\frac{y}{\sigma_{y}}, \tan ^{-1} \frac{\sigma S}{\sigma_{y}}\right) d_{y}
$$

Note on the curves of ef(a, $Y)$ in Appendix $C$ that $i f y / \sigma_{y}=d$ is not larger than 2 or 3 , the usual case, we can approximate this integral by a trapezoid

$$
N_{k}=4 B I_{o} d \operatorname{ef}\left(\frac{d}{2 \sigma_{y}}, \tan ^{-1} \frac{\sigma s}{\sigma_{y}}\right)
$$

(If the magnification is $M$ the $d$ of $(110)$ and (II1) is replaced by $d / M$.$) On the curve of e f(0, y)$ in Appendix $C$ it will be seen that for $\tan Y=\sigma s / \sigma_{y}$ greater than 1 or 2 the proportionate increase of flux density with increase of $s$ diminishes rapidly. The $y^{\prime}$ distribution is given by

$$
\mathrm{dN}_{k}\left(\mathrm{y}^{\prime}\right)=2 B I_{o} d y^{\prime} \frac{\sigma s}{\sqrt{\sigma_{y^{\prime}}+\sigma^{2}}} e^{-\frac{1}{2} y^{\prime 2} /\left(\sigma_{y^{\prime}}+\sigma^{2}\right)}
$$

and will be magnified by $1 / M$. In the example this has all been passed by the slit; the angular variation is determined by $\sigma_{y^{\prime}}$ and $\sigma$. We can usually make $\sigma_{\mathrm{y}^{\prime}}<\sigma$. The radiation angle $\sigma$ is roughly proportional to $(1 / \gamma)\left(\lambda / \lambda_{c}\right)^{\frac{1}{2}}$ from $(52)$ and $\lambda_{c}=4 \pi \rho / 3 \gamma^{3}$. Combining these, at a given $\lambda, \sigma$ is approximately proportional to $(\gamma / \rho)^{\frac{1}{2}}$ or to $B^{\frac{1}{2}}$ (this $B$ is magnetic field). Flux constant $B$ is (75) proportional to $\gamma^{2} / \rho$ and to $\mathrm{H}_{2}$ which is slowly varying from $\lambda / \lambda_{c}$ ranging from say 0.3 to 20 . 
In general we need to keep $\sigma_{y}$ and $\sigma_{y^{\prime}}$ sma11 (sma11 emittance) and to work with $Y$ and $B$ as high as feasible, without encountering serious beam power problems. Angular variation in the slice through the slit of Fig. 29 can be reduced by diaphragming further on in the system, but this of course reduces the flux.

In the $\mathrm{x}$ direction, along the slit, the angular density is given by (79) multiplied by $1 / M$. The $x^{\prime}$ extent is simply equal to the orbital arc subtended $\times 1 / M$. The size $x$ has a one $\sigma$ width (80) of $\left(\sigma_{x}^{2}+s^{2} \sigma^{2}\right)^{\frac{1}{2}} \times M$, and this indicates the desirability of keeping $\sigma_{x}$ and $s$ sma 11 .

There are many different focusing systems that can precede or follow the slit. They are all subject to the unfortunate restriction that we camnot compress phase space; we cannot reduce both size and angle witiout slicing off flux. Interaction with the numerous source parameters is complex and analys is is probably best done numerically. 
1. J. Schwinger, Phys. Rev. 75, 1912 (1949).

2. A.A. Sokolov and I.M. Ternov, "Synchrotron Radiation", Pergamon Press (1968).

3. D.H. Tomboulian and P.I. Hartman, Phys. Rev. 102, 1423 (1956).

4. R.A. Mack, "Spectral and Angular Distributions of Synchrotron Radiation". Internal report CEAL-1027, Cambridge Electron Accelerator (1966).

5. K. Codling, Rep. Prog. Phys. 36, 541 (1973).

6. E.M. Rowe, IEEE Trans, on Nuc. Sci. NS-20, 973 (1.973).

7. Orsay LURE Group, Ann. de Phys. 9, 9 (1975).

8. H.E1lis and J.R. Stevenson, "Computer Calculation and Numerical Tabulations of Some MacDonald Functions", School of Physics, Georgia Institute of Technology。

9. M. Abramowitz and Irene Stegun, "Handbook of Mathematical Functions", Dover (1972).; also U.S. Government Printing office (1972)。

10. A. BIanc-Lapierre and R. Fortet, "Theory of Random Functions", Gordon and Breach (1965); C.E. Weatherburn, "Mathematical Statistics", Cambridge Univ. Press (1949).

11. B.O. Peirce, "A Short Table of Integrals", Ginn and Co. (1929)。

12. J.W. BIaker, "Geometric Optics", Marcel Dekker (1971)。

13. W. Brouwer, "Matrix Methods in Optical Instrument Design", Benjamin (1964)。

14. A.P. Sabersky, Particle Accelerators 5, 199 (1973).

15. A.P. Banford, "Transport of Charged Particle Beams", Spon (1966).

16. E.D. Courant and H.S. Snyder, Annals of Phys. 3, 1 (1958); G.K. Green and E.D. Courant, "The Proton Synchrotron", Handbuch der Physik XIIV, Springer (1959);

H.Bruck, "Accelerateurs Circulaires de Particules", Presses Universitajres de France (1966);

M. Sands, "Physics of Electron Storage Rings", SLAC Report 121 (1970). 


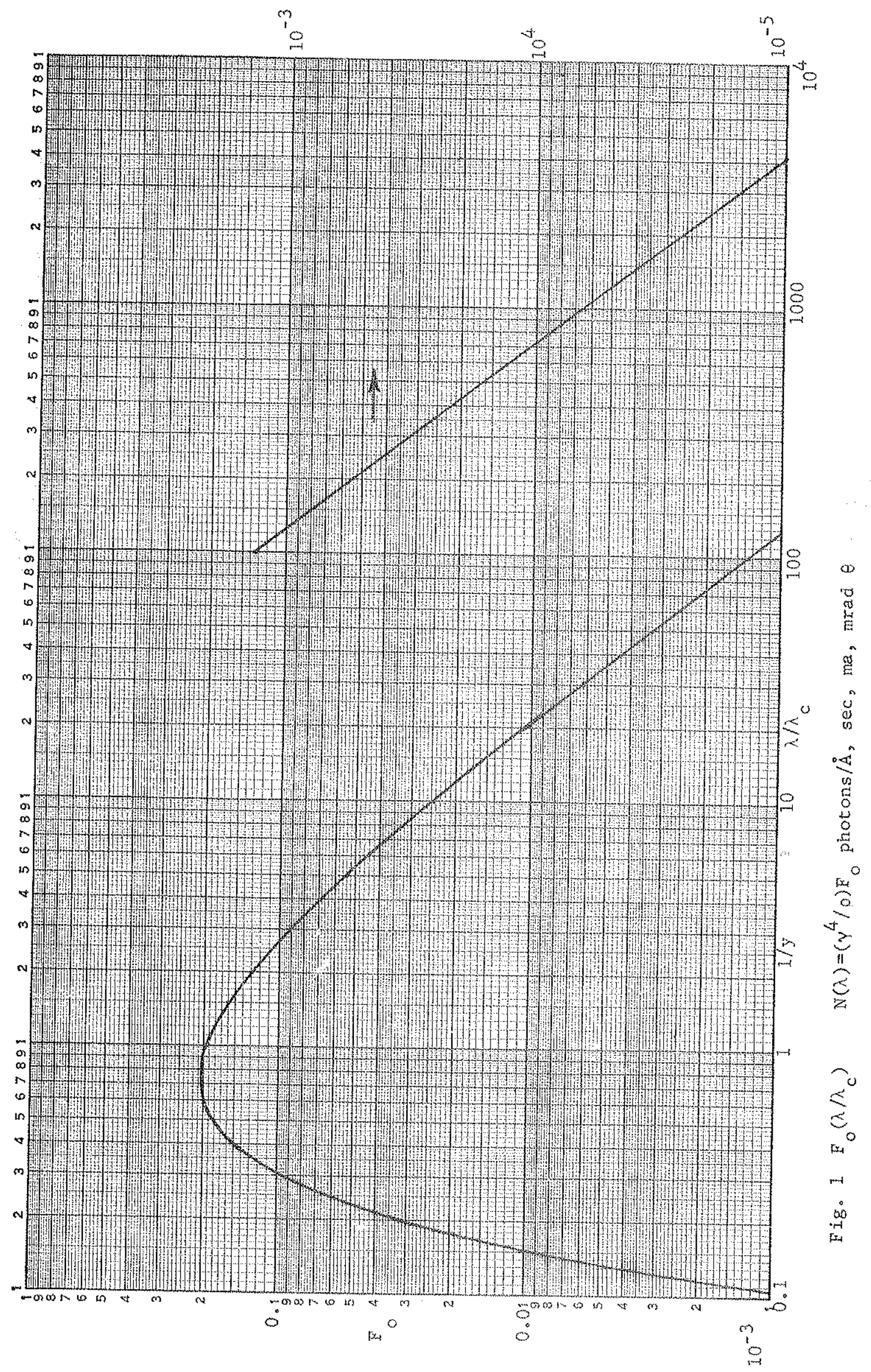




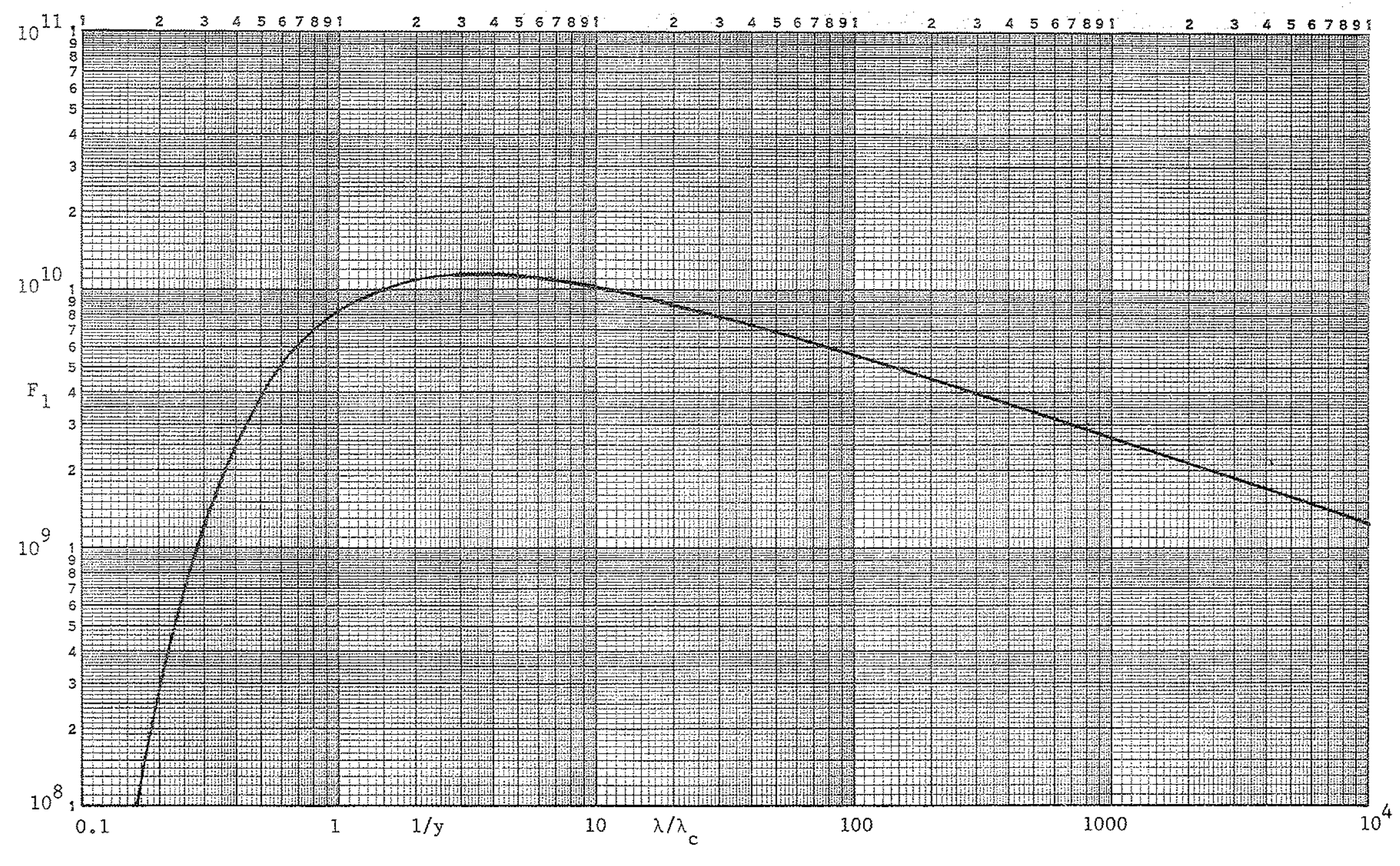

Fig. $2 \mathrm{~F}_{1}\left(\lambda / \lambda_{\mathrm{c}}\right) \quad \mathrm{N}_{\mathrm{k}}(\lambda)=\mathrm{k} \gamma \mathrm{F}_{1}$ photons $/ \mathrm{k} \lambda$, sec, ma, mrad $\theta$ 


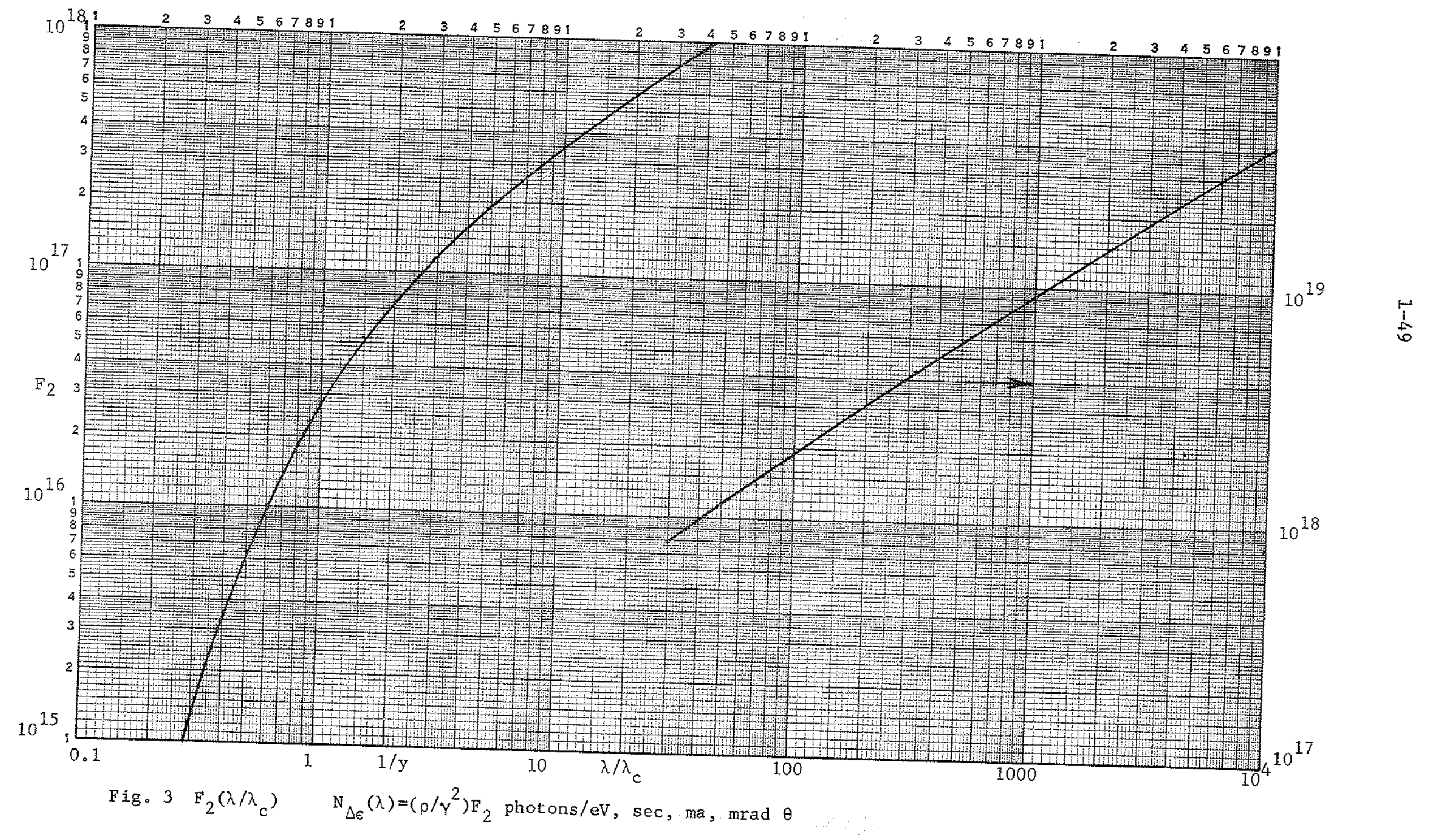




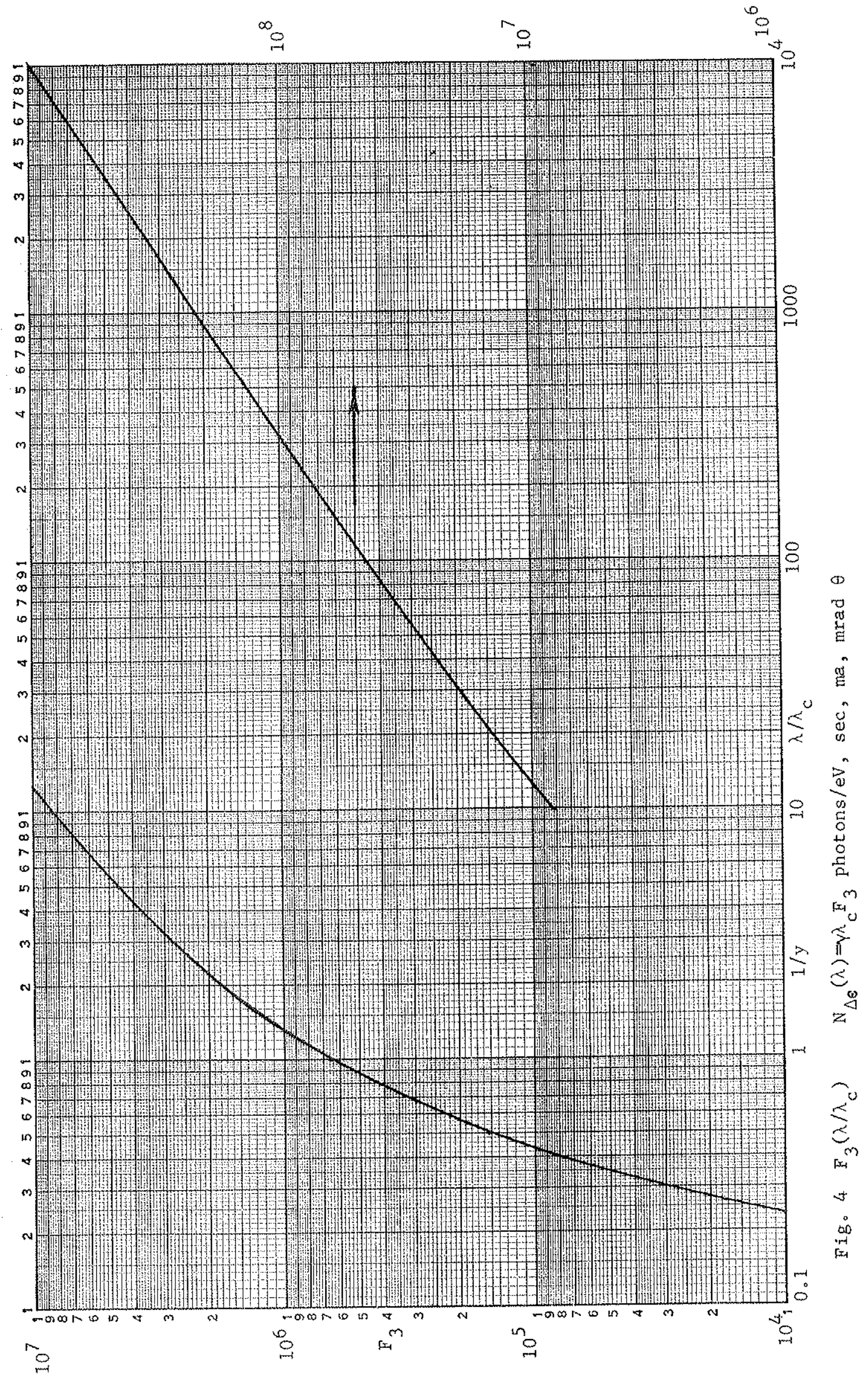




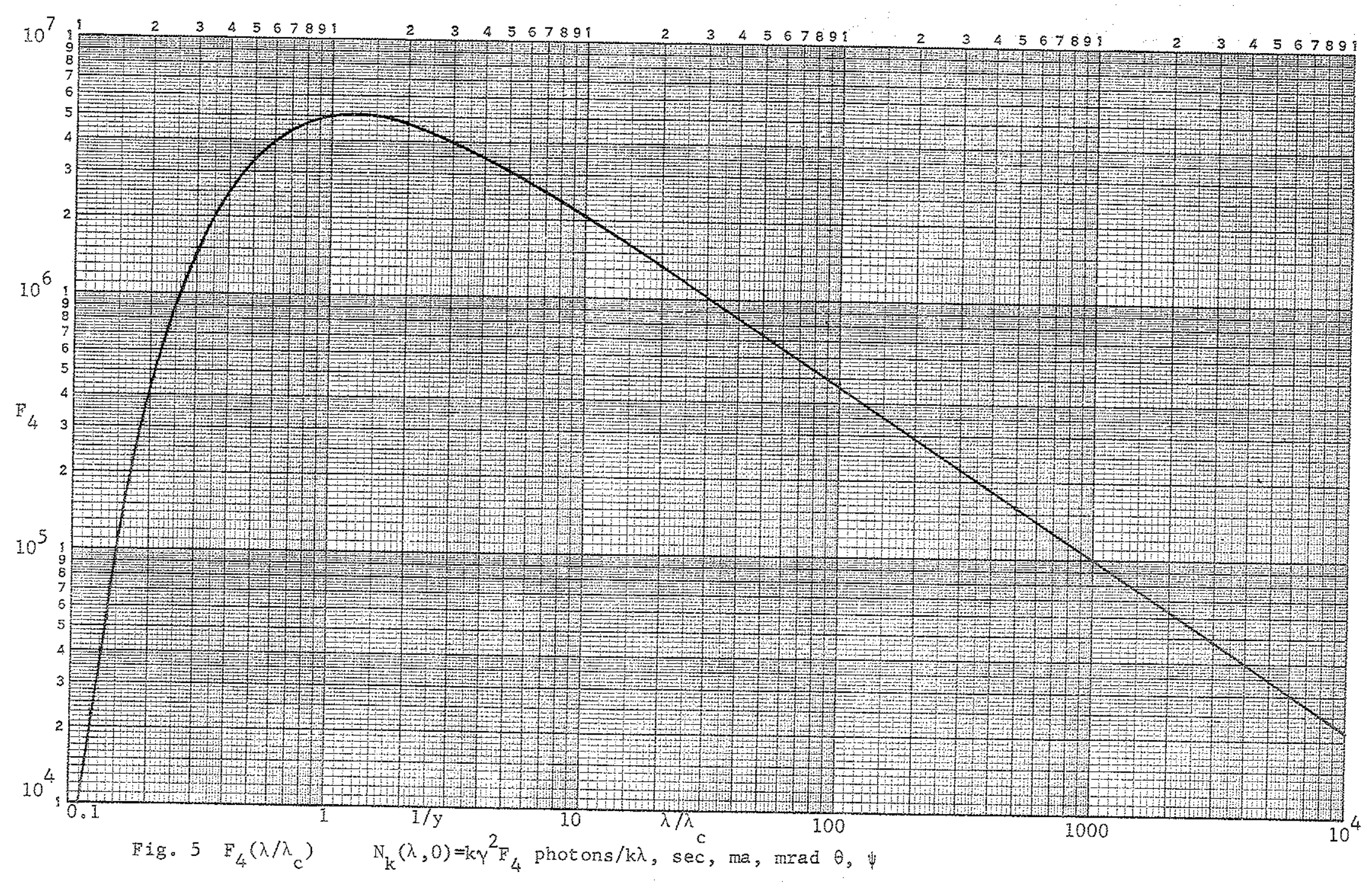




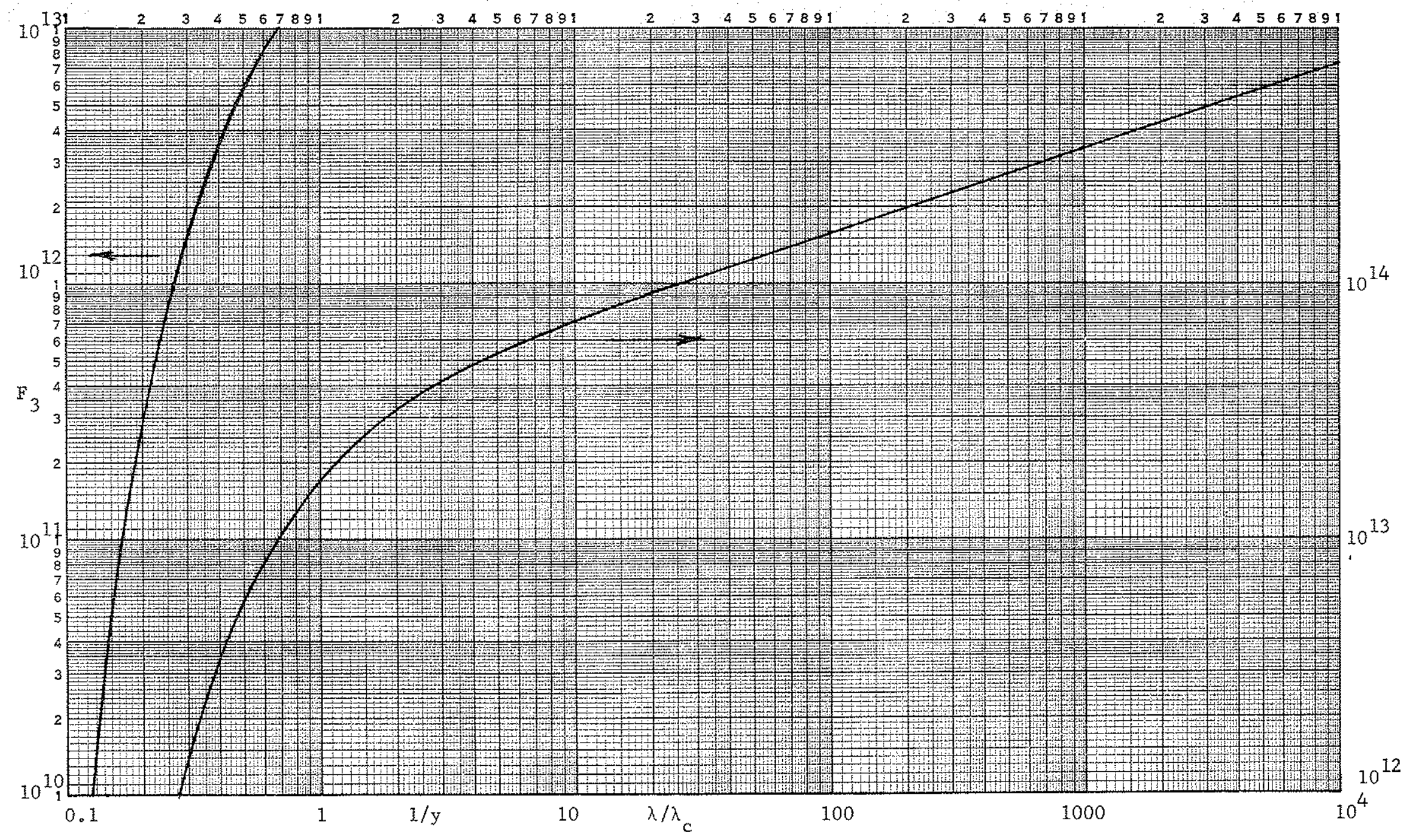

Fig。 $6 F_{5}\left(\lambda / \lambda_{c}\right) \quad N_{\Delta e}(\lambda, 0)=(\rho / \gamma) F_{5}$ photons/eV, sec, ma, mrad $\theta$, 


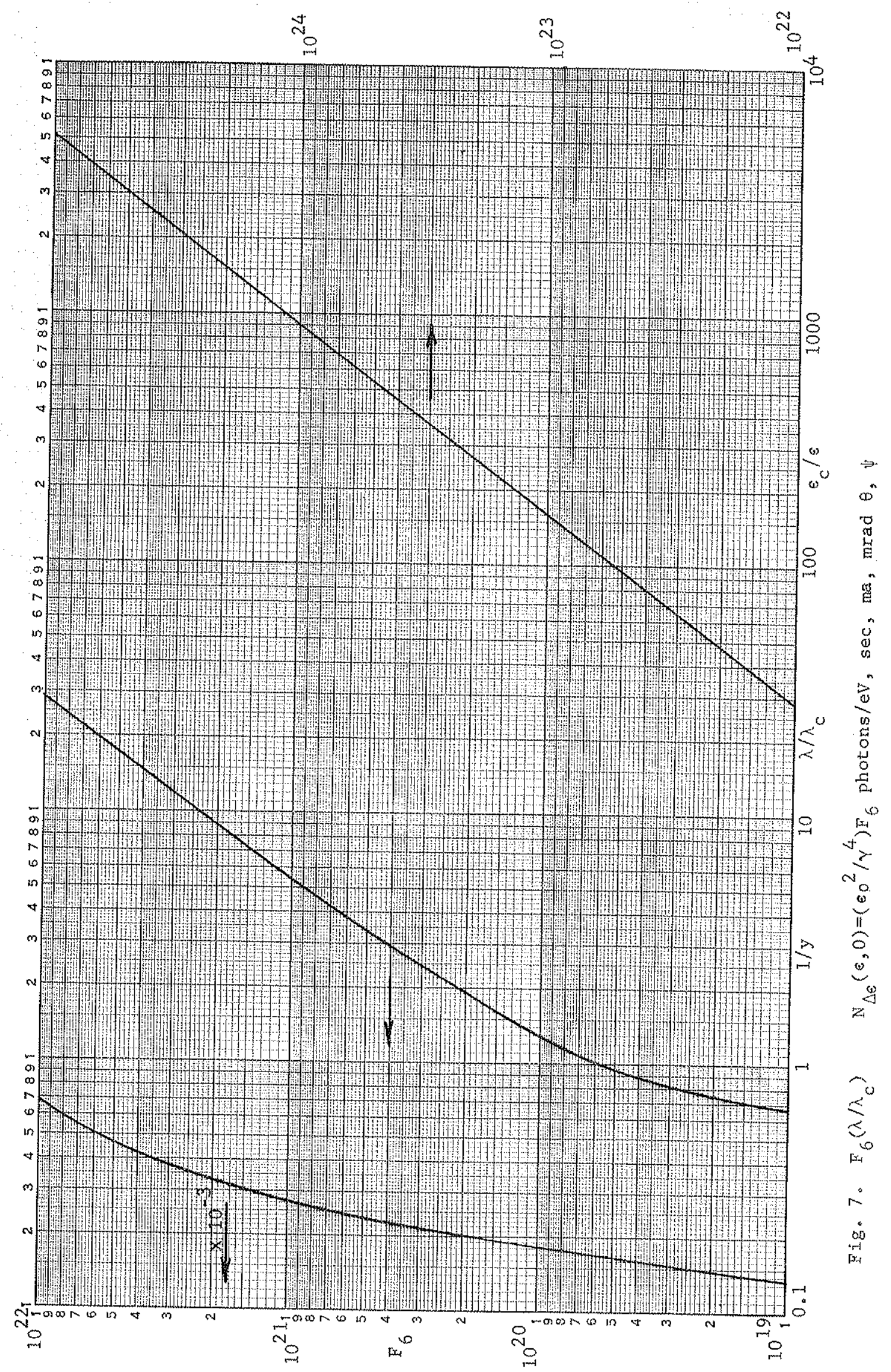




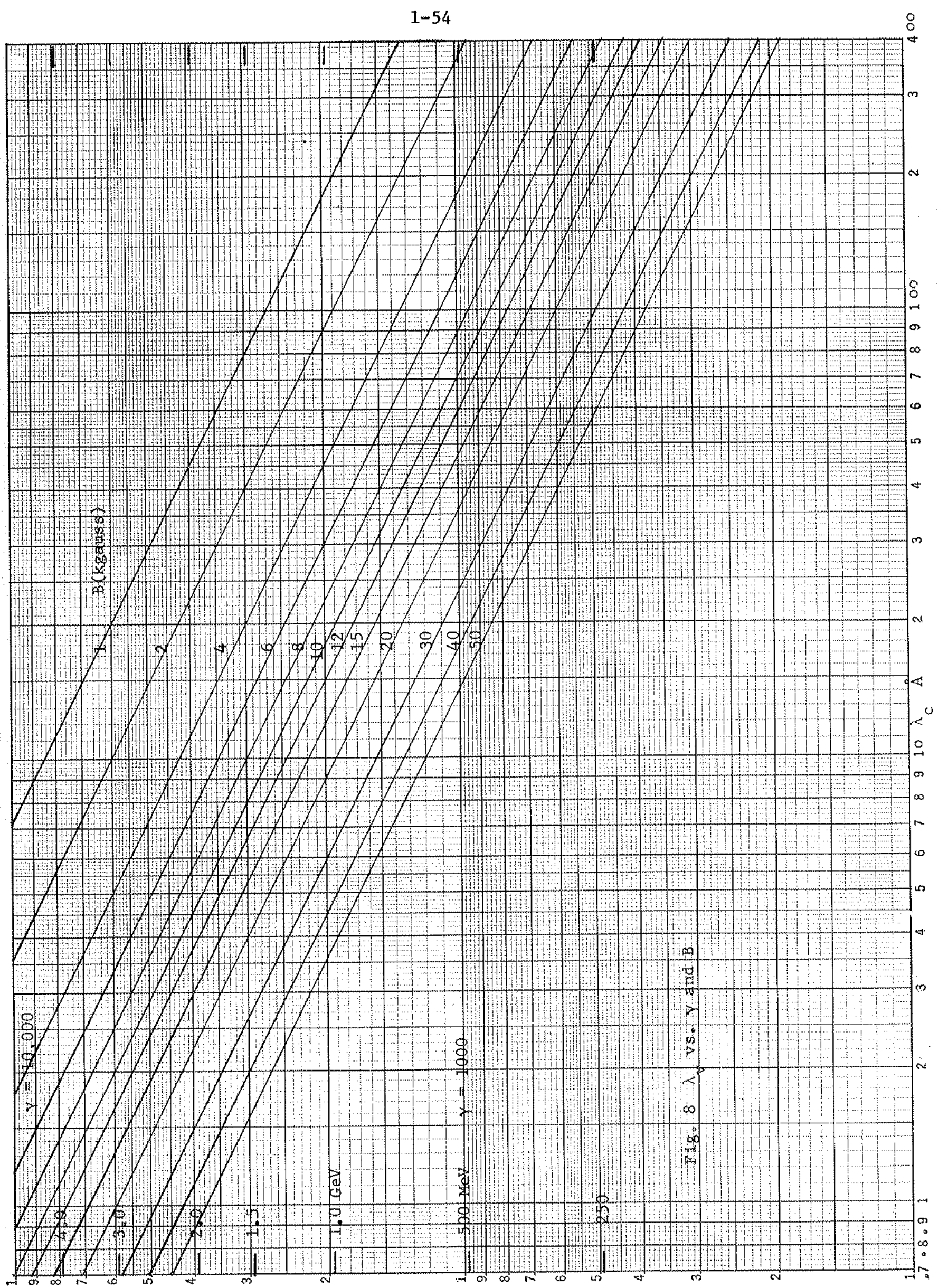


1-55

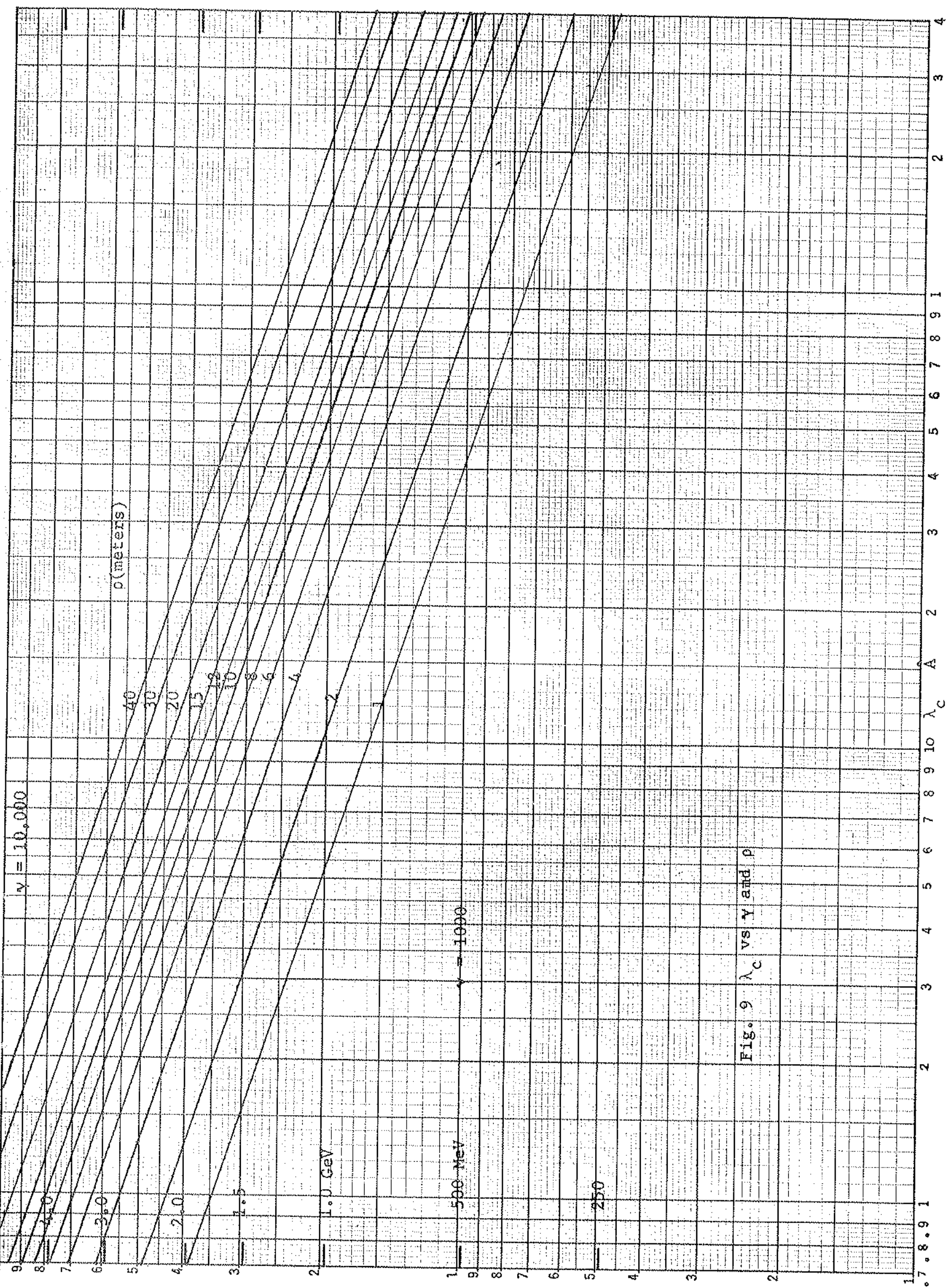


1. -56

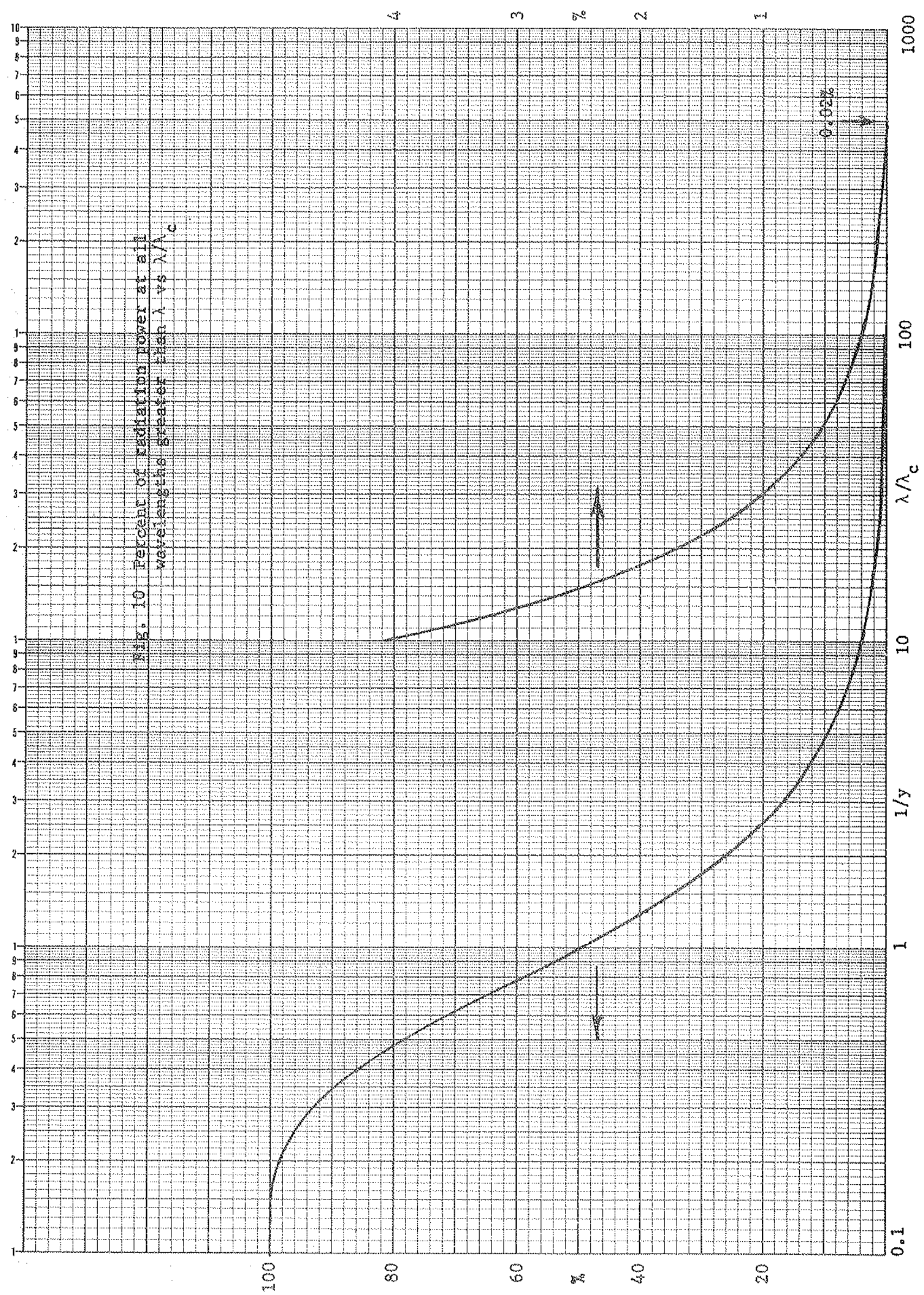




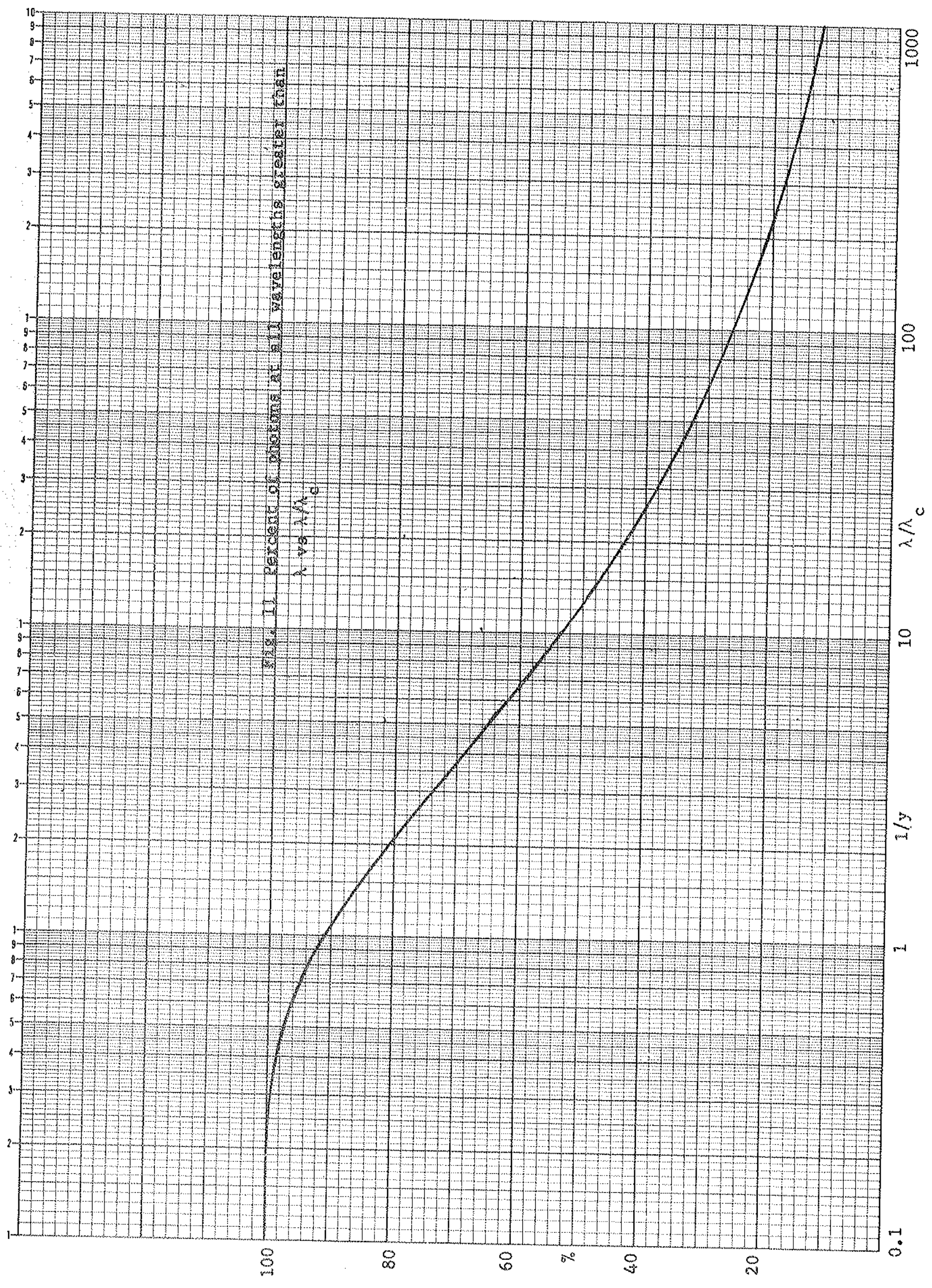




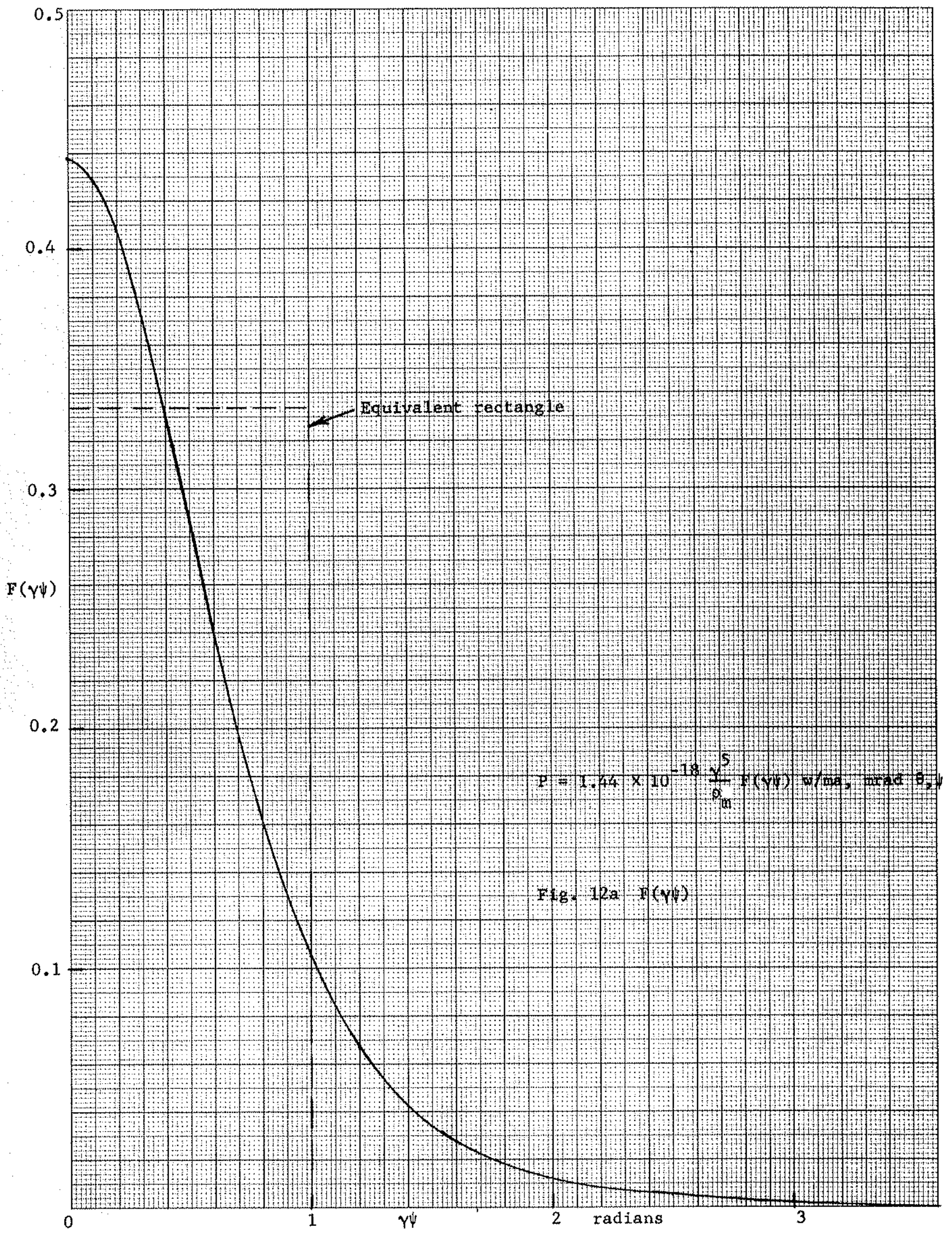




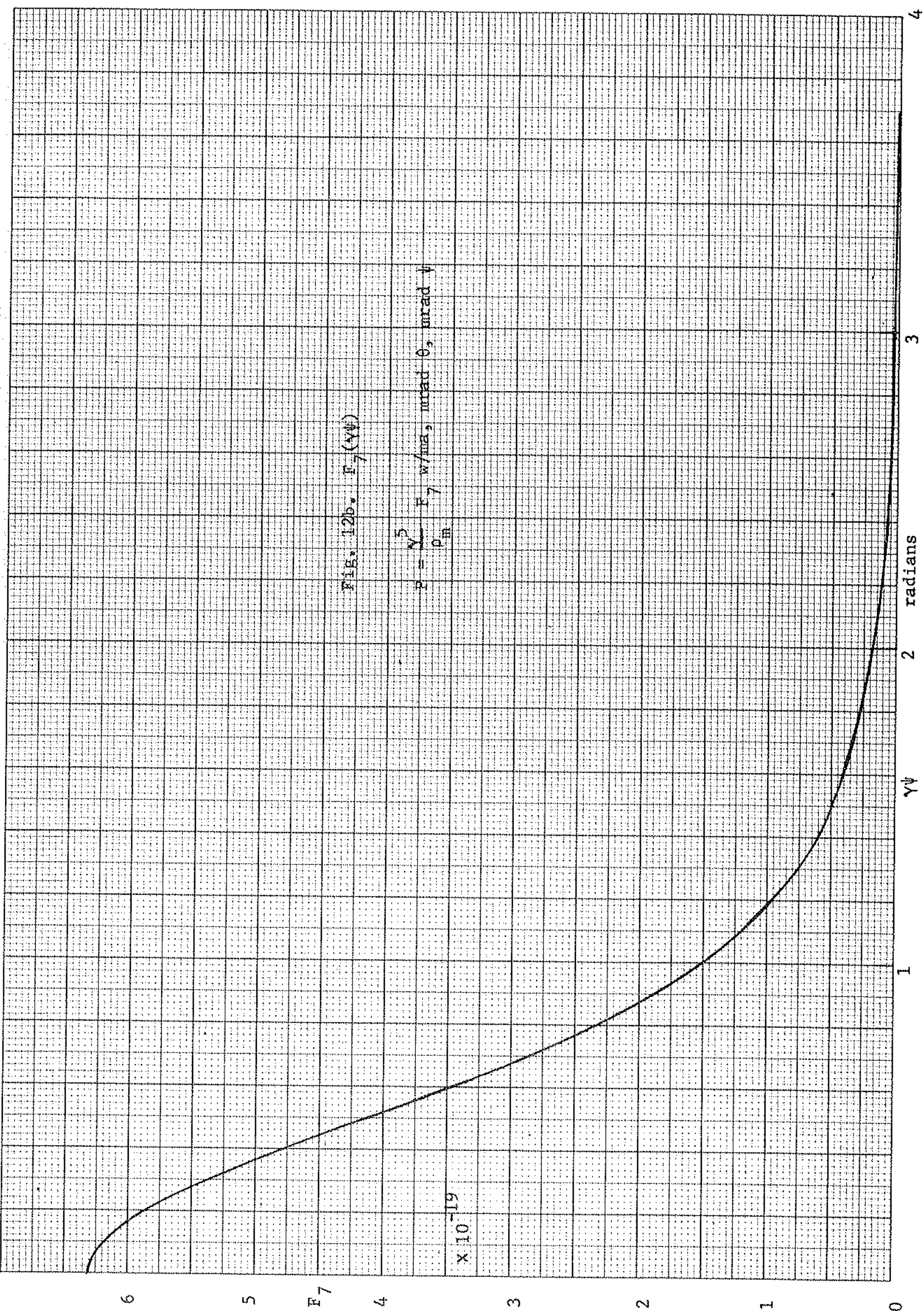




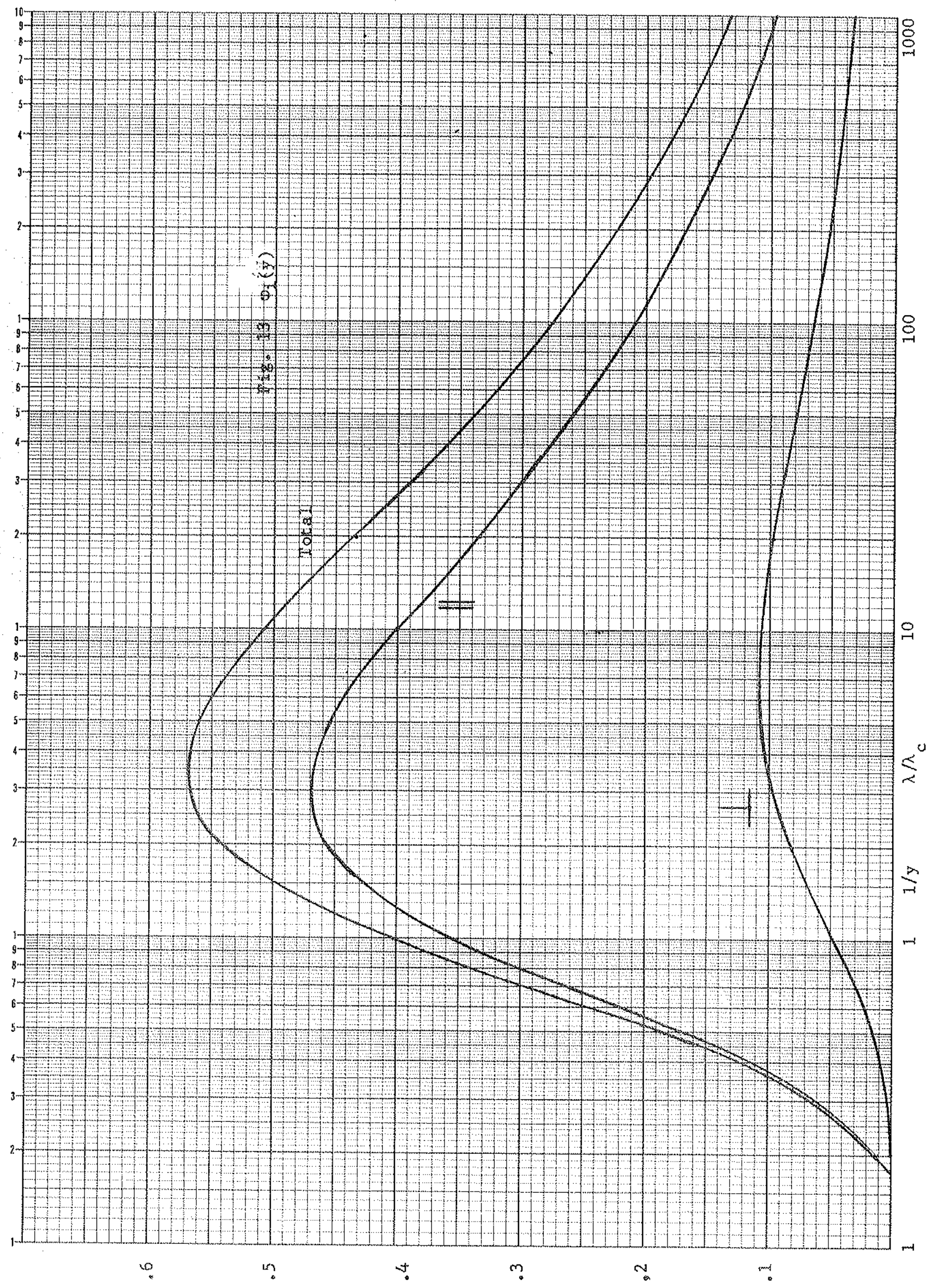




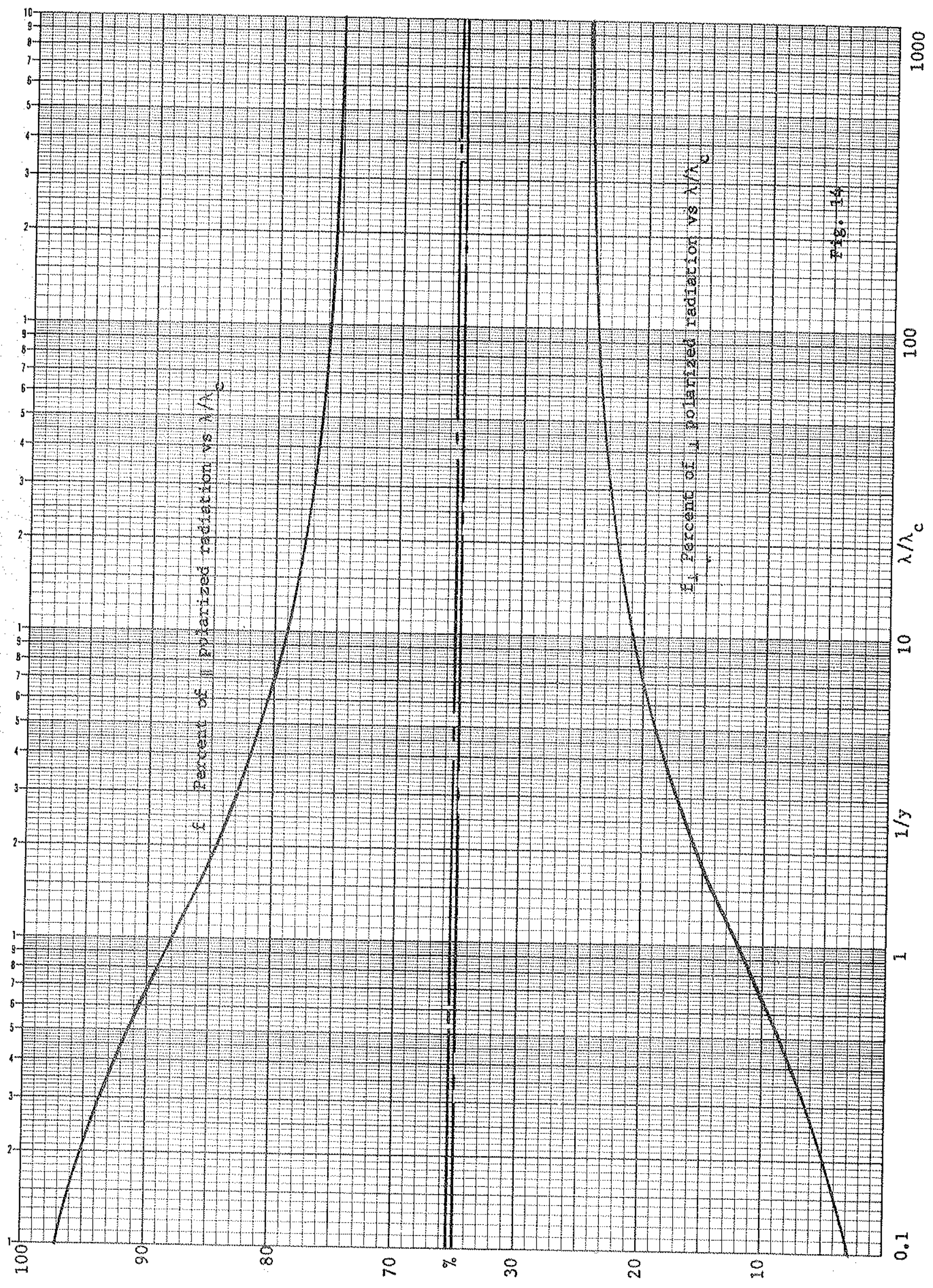




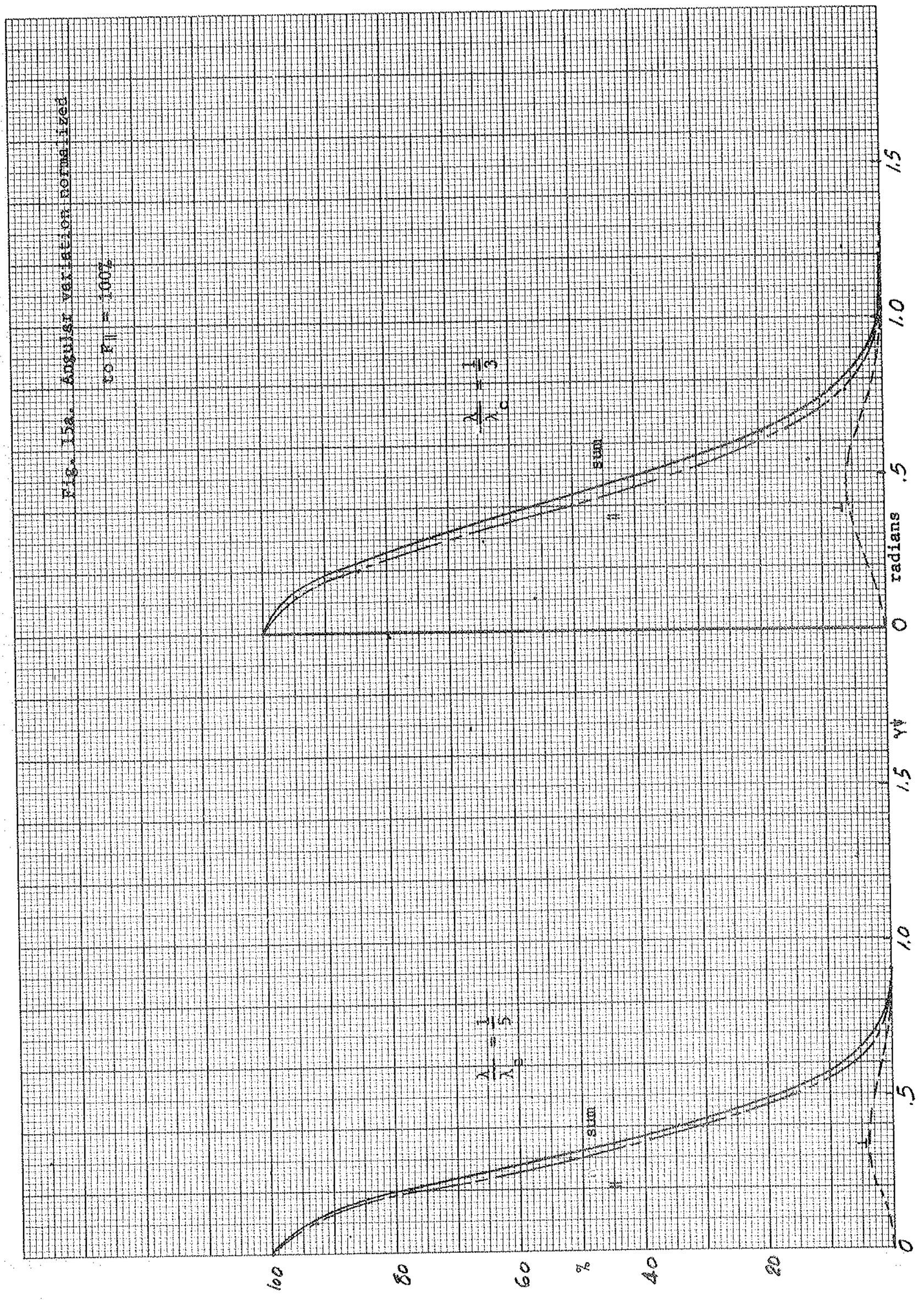




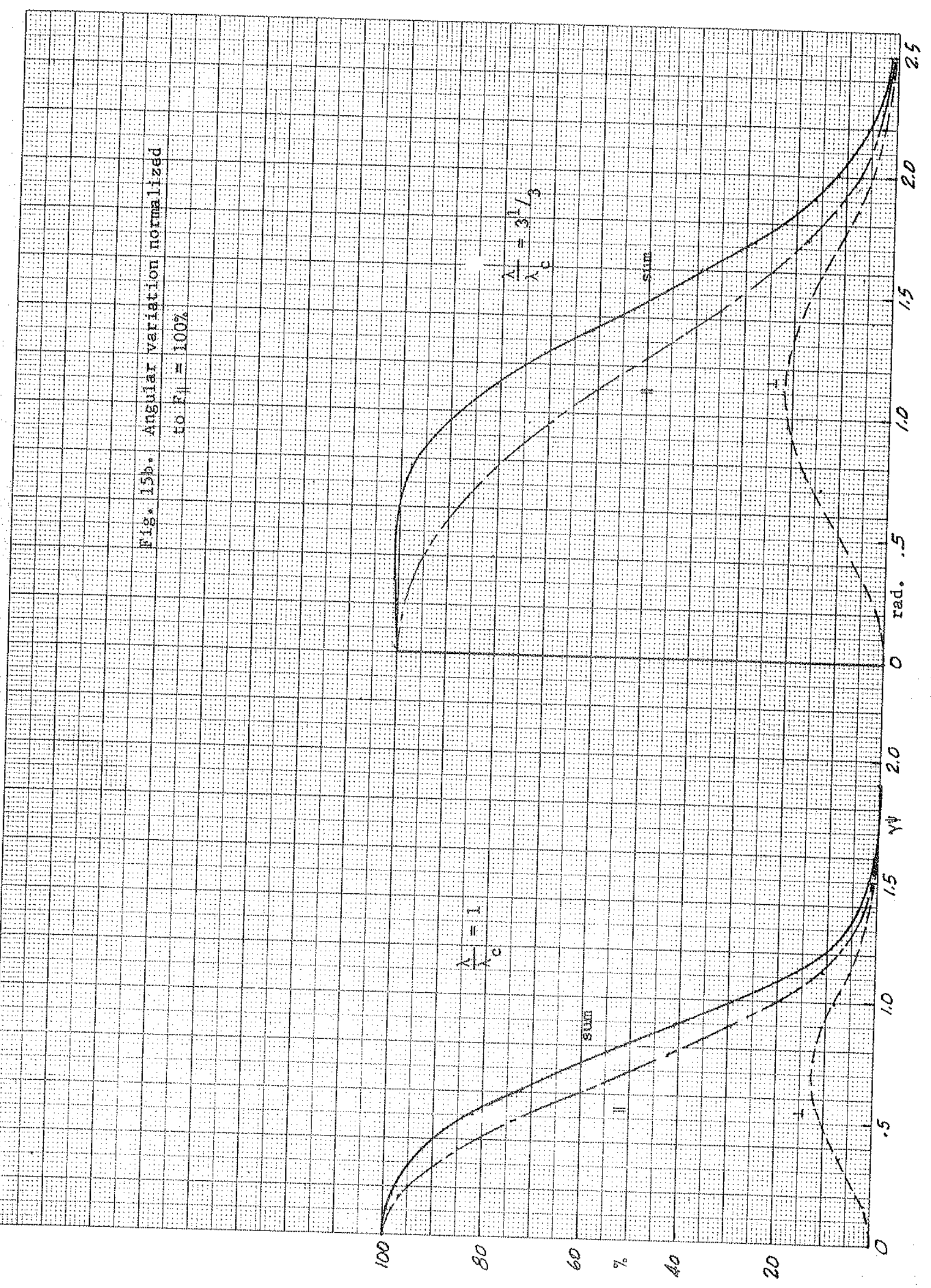




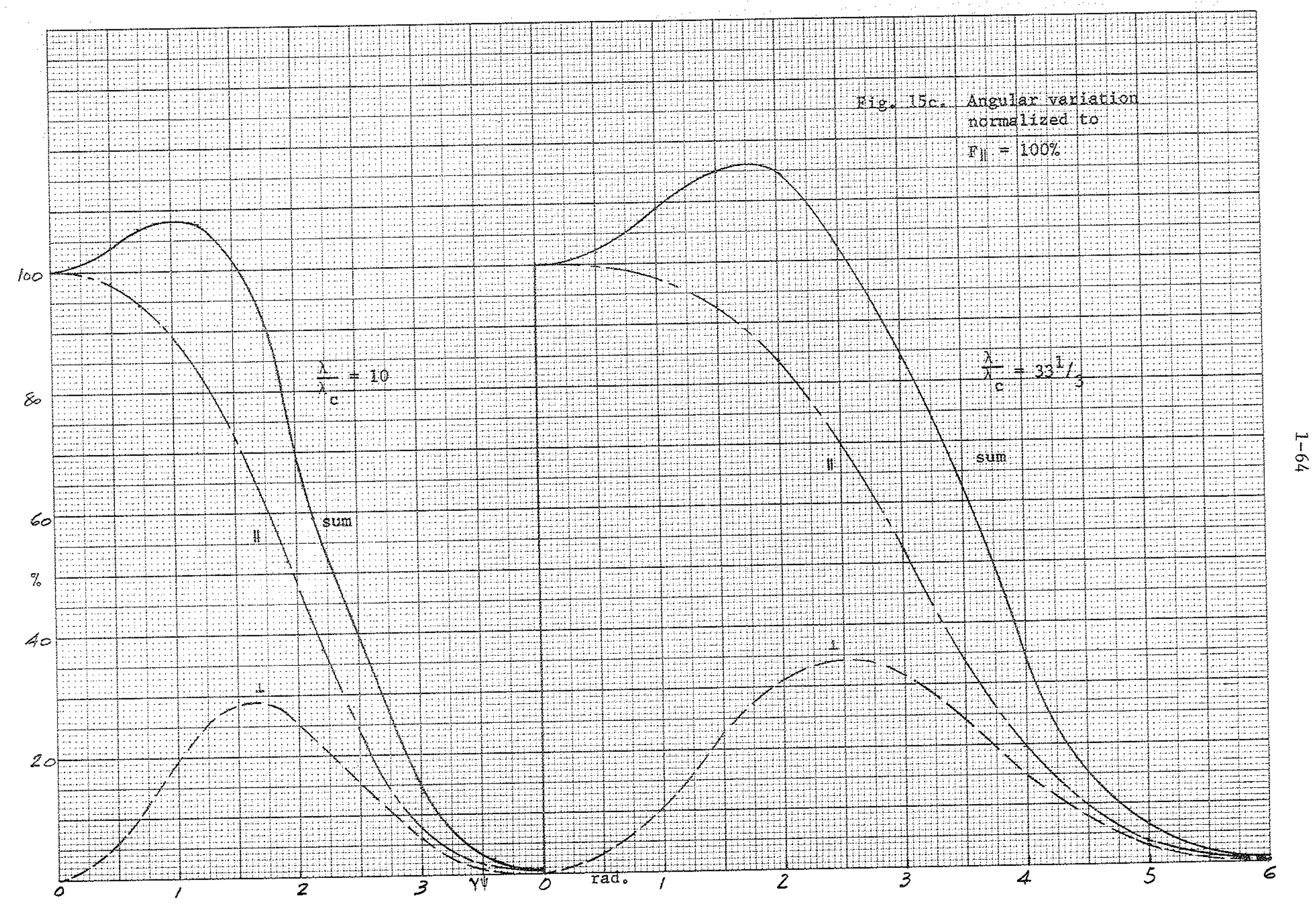




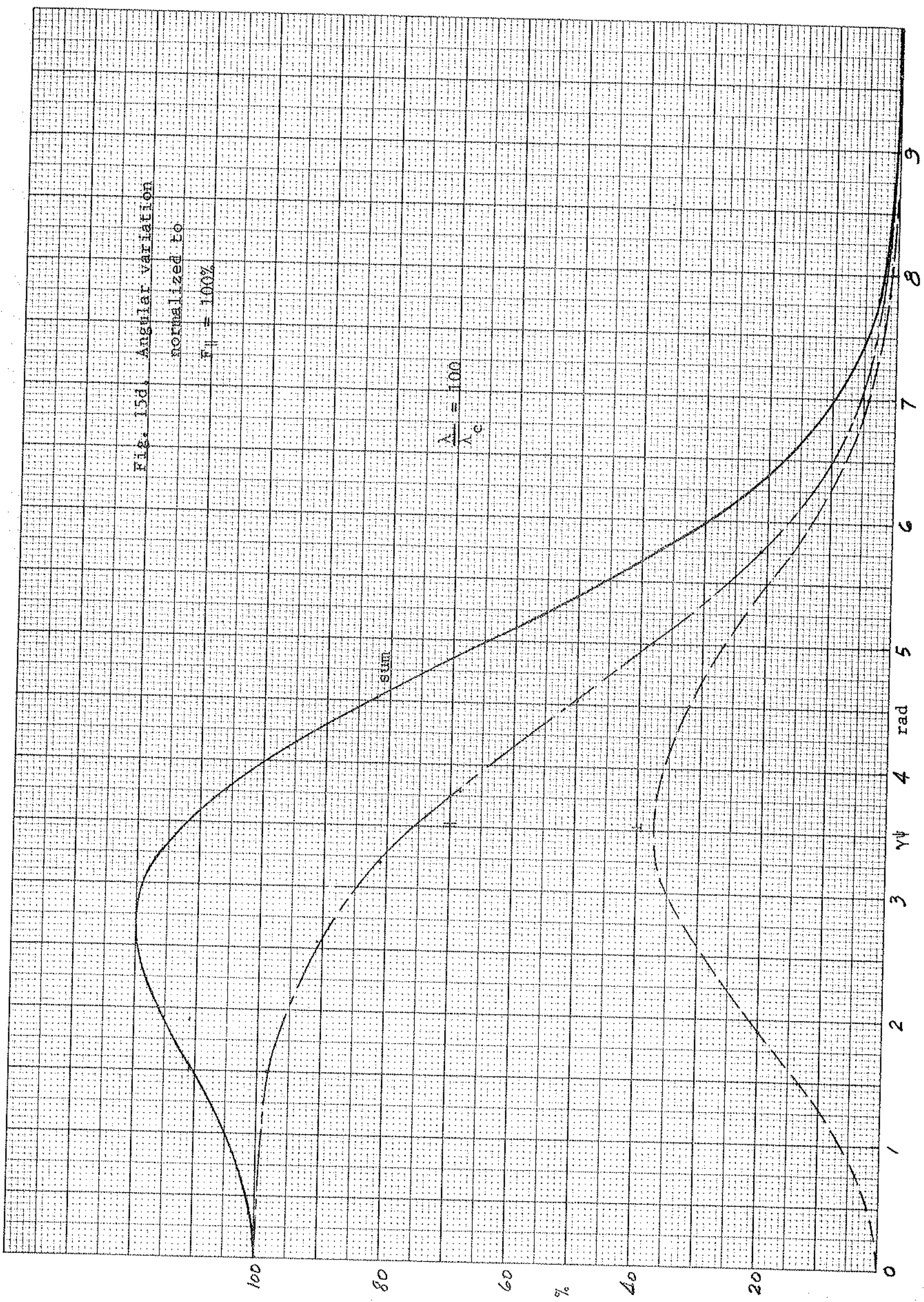




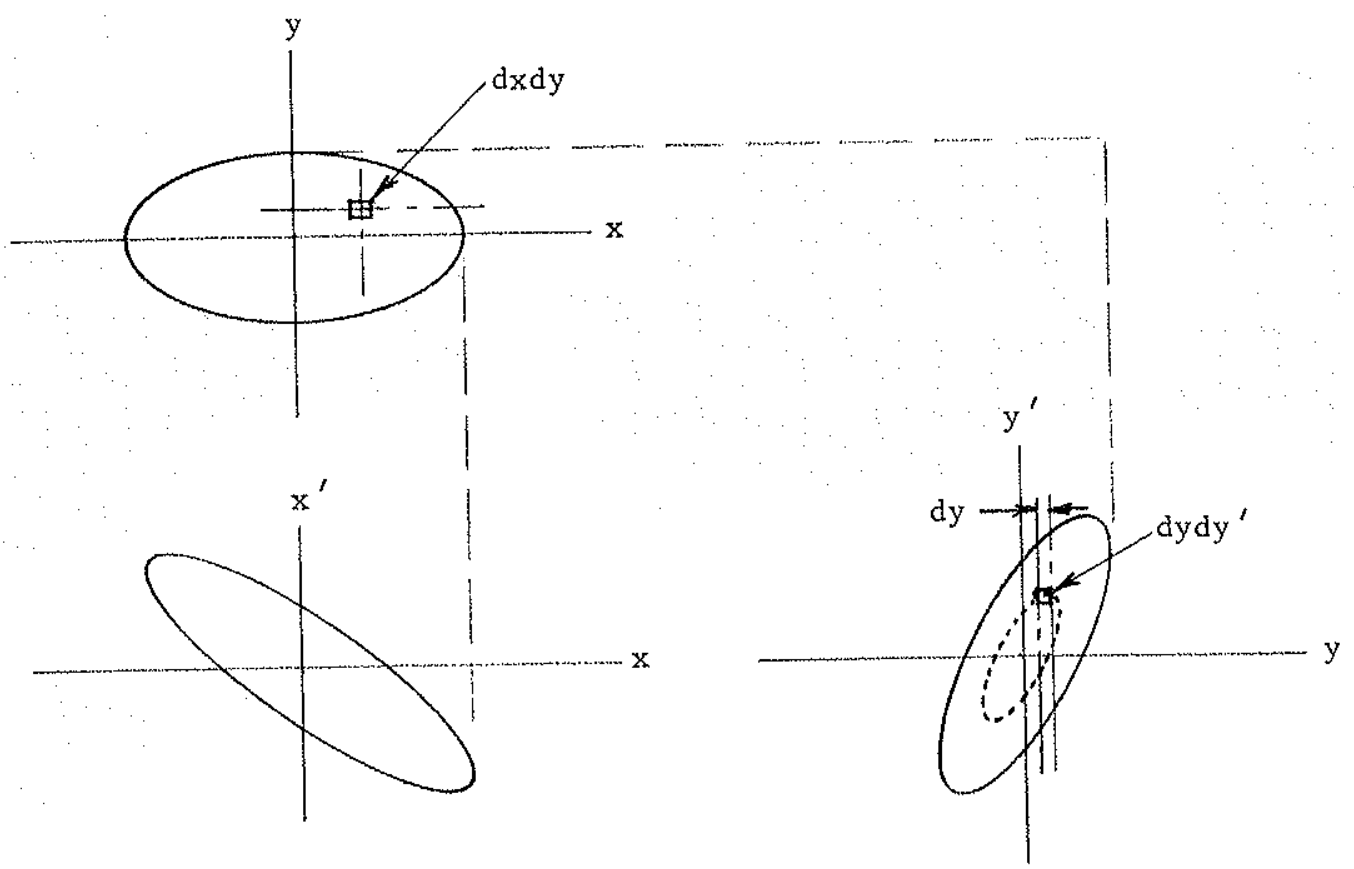

Fig. 16 Electron beam cross section in configuration and phase space

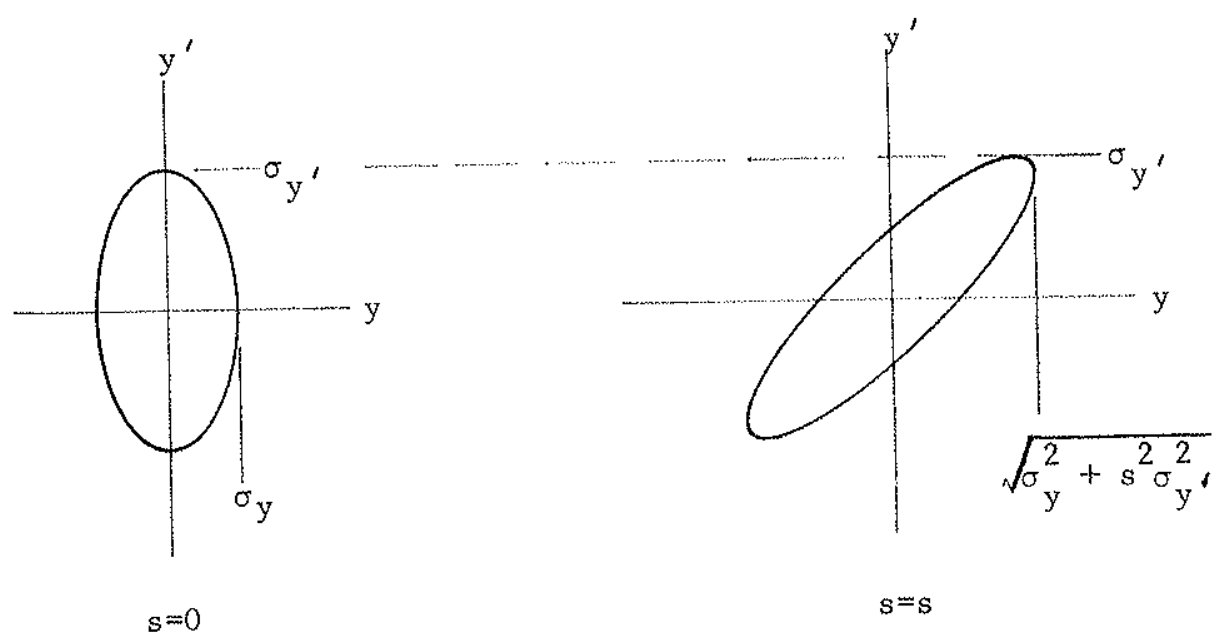

Fig. 17a. Envelope at waist, $s=0$. b. Envelope at $s$. 


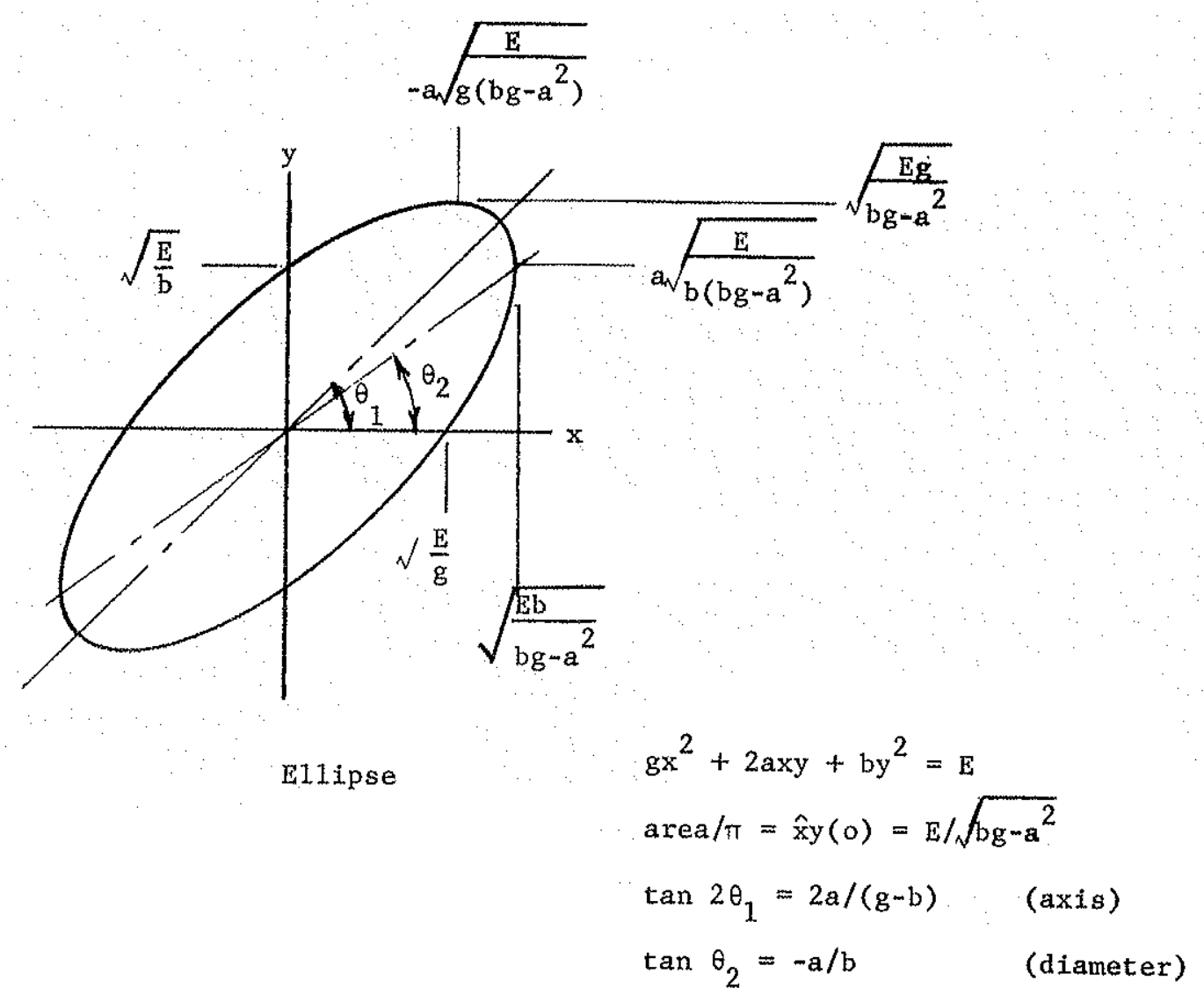

Fig. 18 Properties of ellipse

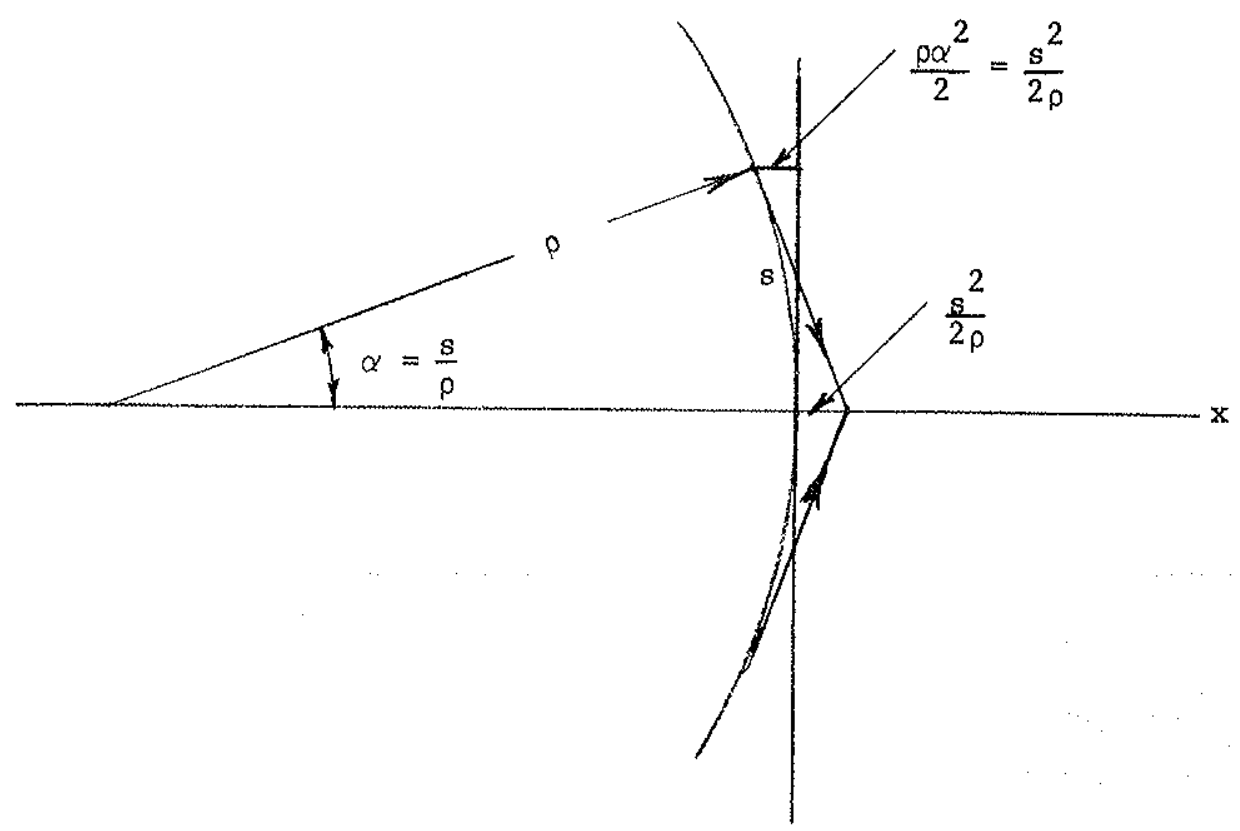

Fig. 19 Arc in $x$ w plane 


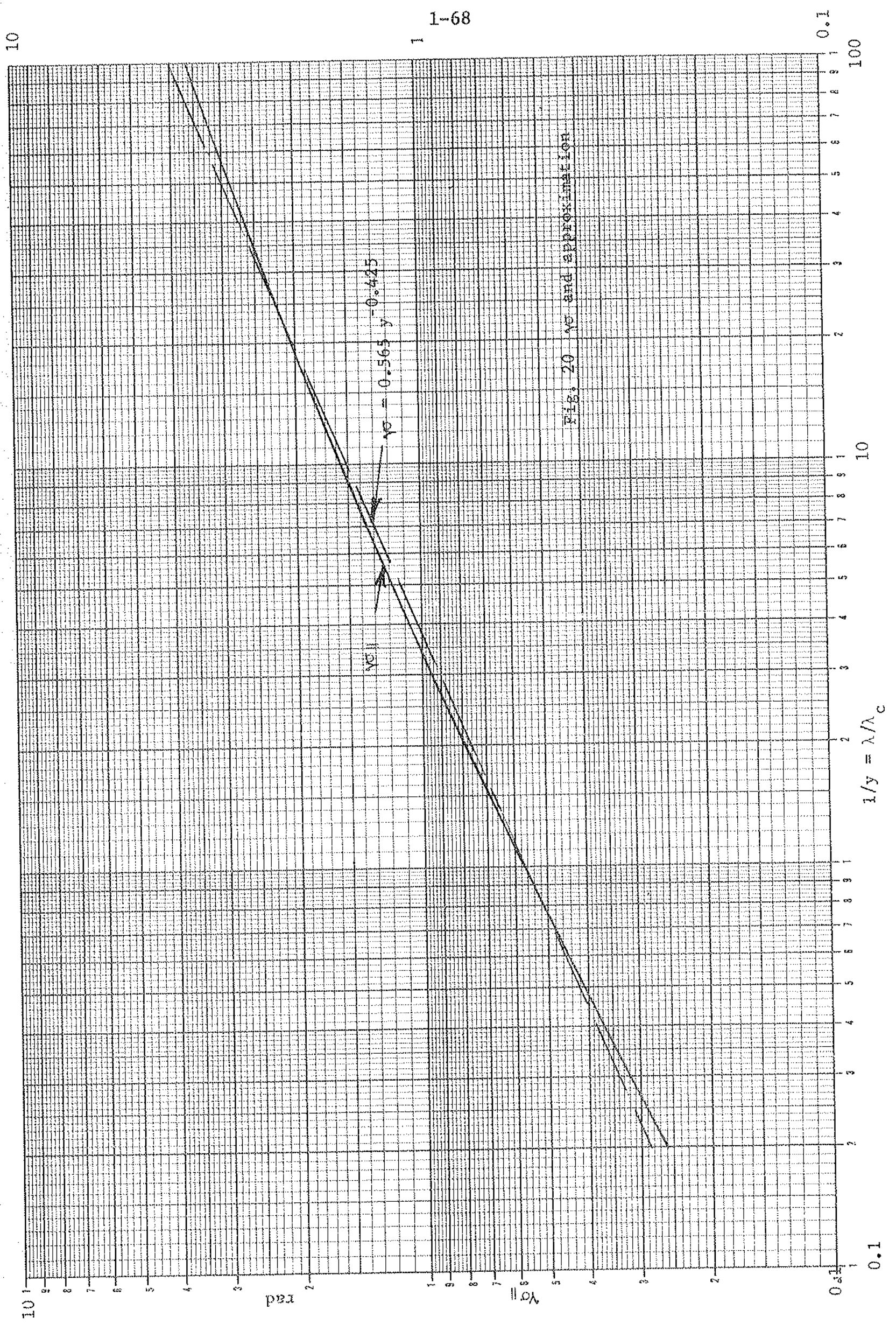




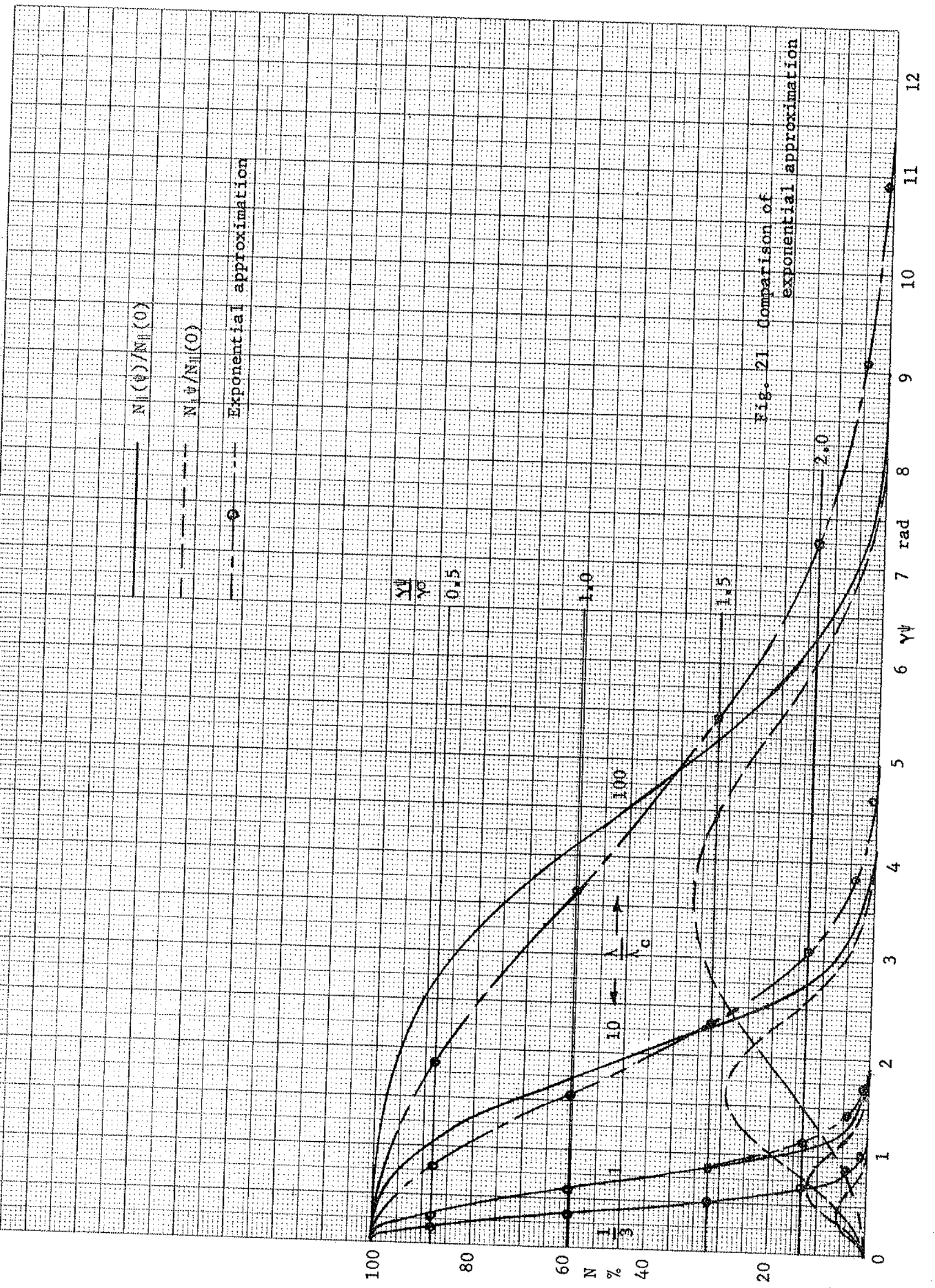


APPENDIX A

Computation of synchrotron radiation spectra requires the modified Bessel functions $\mathrm{K}_{1 / 3}(\mathrm{x}), \mathrm{K}_{2 / 3}(\mathrm{x}), \mathrm{K}_{5 / 3}(\mathrm{x})$ and the integral $\int_{x}^{\infty} \mathrm{K}_{5 / 3}(\eta) \mathrm{d} \eta$, or the Tomboulian and Hartman $G=x^{3} \int_{x}^{\infty} k_{5 / 3}(\eta)$ d $\eta$. The most accurate tables are probably the "Tables of Besse1 Functions of Fractional Order," Vol. II, Computation Lab., National Bureau of Standards, Columbia University Press, New York, 1948. However, the $\mathrm{K}_{\nu}$ functions must be computed from the $I_{V}$, which are tabulated, using;

$$
K_{\nu}(x)=\frac{\pi}{2 \sin \pi y}\left[I_{-V}(x)-I_{\nu}(x)\right]
$$

To obtain $\mathrm{K}_{5 / 3}(\mathrm{x})$ from these tables it would be necessary to use a recurrence relation such as

$$
k_{5 / 3}(x)=k_{1 / 3}(x)+\frac{4}{3 x} k_{2 / 3}(x)
$$

These tables give $x^{ \pm \nu} I_{F \nu}(x)$ for small arguments and since this product converges as $x \rightarrow 0$ the values of $K$ for arguments less than 0.002 (less than 0.005 for four figure accuracy) can be calculated by

$$
\begin{aligned}
& K_{1 / 3}(x)=1.81380\left(0.930437 x^{-1 / 3}-0.888823 x^{1 / 3}\right) \\
& K_{2 / 3}(x)=1.81380\left(0.592549 x^{-2 / 3}-0.697828 x^{2 / 3}\right)
\end{aligned}
$$

Values of $G_{0}$ for arguments less than 0.001 can be obtained by use of Schwinger's expansion for $\omega \ll \omega_{c}$. Equating his III6 and II20

$$
G_{0}=\int_{y}^{\infty} k_{5 / 3}(\eta) d \eta=2.149 y^{-2 / 3}\left[1-0.677(y / 2)^{1 / 3}+\ldots\right], y<0.001
$$


The most convenient tables of $\mathrm{K}_{1 / 3}, \mathrm{~K}_{2 / 3}, \mathrm{~K}_{5 / 3}$ and $\mathrm{G}$ are in an unnumbered and undated report ${ }^{8}$ - H. Ellis and J.R. Stevenson, "Computer Calculations and Numerical Tabulations of Some MacDonald Functions," School of Physics, Georgia Tech. (There is a misprint on page 3. The approximation for $x \gg 1$ is $k_{\mu}(x)=\sqrt{\frac{\pi}{2 x}} e^{-x}$ ).

The short tables and graphs in the report by R.A. Mack ${ }^{4}$ are convenient but the report, issued from the late Cambridge Electron Accelerator, is hard to get. Note that Mack's $g(r)$ is related to $G_{1}$ by

$$
g(r)=G_{1}(r) / 0.9 \times 2^{2 / 3}(5 / 3) !=G_{1}(r) / 2.150
$$

The following short Table AI of functions has arguments spaced for reasonable interpolation. $H_{0}(y, 0)=k_{2 / 3}^{2}(y / 2)$ and $G_{o}(y)=\int_{y}^{\infty} K_{5 / 3}(\eta) d \eta$ are tabulated. $H_{i}$ and $G_{i}$ can then be obtained by multiplying by $y^{i}$.

Figure $A 1$ is a graph of $G(y)$ e.g. $G_{3}(y)$ vs. $y$. 
TABLE AI

\begin{tabular}{ll}
$\mathrm{y}$ & $\mathrm{K}_{1 / 3}(\mathrm{y})$ \\
\hline & \\
.0001 & 36.284 \\
.001 & 16.715 \\
.002 & 13.192 \\
.004 & 10.376 \\
.006 & 8.995 \\
.008 & 8.116 \\
.010 & 7.486 \\
.020 & 5.781 \\
.030 & 4.932 \\
.040 & 4.386 \\
.050 & 3.991 \\
.060 & 3.685 \\
.070 & 3.437 \\
.080 & 3.231 \\
.090 & 3.054 \\
.100 & 2.900 \\
.150 & 2.343 \\
.200 & 1.979 \\
.250 & 1.714 \\
.300 & 1.509 \\
.350 & 1.343 \\
.400 & 1.206 \\
.450 & 1.809 \\
.500 & $9.890-1$ \\
.550 & $9.018-1$ \\
.600 & $8.251-1$ \\
.650 & $7.571-1$ \\
.700 & $6.965-1$ \\
.750 & $6.422-1$ \\
.800 & $5.932-1$ \\
.850 & $5.489-1$ \\
.900 & $5.086-1$ \\
1.00 & $4.384-1$ \\
1.25 & $3.079-1$ \\
1.50 & $2.202-1$ \\
1.75 & $1.594-1$ \\
2.00 & $1.165-1$ \\
2.25 & $8.581-2$ \\
2.50 & $6.354-2$ \\
2.75 & $4.727-2$ \\
&
\end{tabular}

\begin{tabular}{l}
$\mathrm{K}_{2 / 3}(\mathrm{y})$ \\
\hline 498.86 \\
107.46 \\
67.686 \\
42.621 \\
32.509 \\
26.820 \\
23.098 \\
14.498 \\
1.017 \\
9.052 \\
7.762 \\
6.837 \\
6.136 \\
5.581 \\
5.130 \\
4.753 \\
3.513 \\
2.802 \\
2.329 \\
1.987 \\
1.725 \\
1.517 \\
1.347 \\
1.206 \\
1.086 \\
$9.828-1$ \\
$8.933-1$ \\
$8.148-1$ \\
$7.455-1$ \\
$6.839-1$ \\
$6.288-1$ \\
$5.794-1$ \\
$4.945-1$ \\
$3.406-1$ \\
$2.402-1$ \\
$1.722-1$ \\
$1.248-1$ \\
$9.132-2$ \\
$6.726-2$ \\
$4.981-2$ \\
\\
4
\end{tabular}

\begin{tabular}{l}
$\mathrm{K}_{5 / 3}(\mathrm{y})$ \\
\hline \\
$6.652+6$ \\
$1.4330+5$ \\
$4.514+4$ \\
$1.422+4$ \\
$7.233+3$ \\
$4.478+3$ \\
$3.087+3$ \\
$9.723+2$ \\
$4.946+2$ \\
$3.061+2$ \\
$2.110+2$ \\
$1.556+2$ \\
$1.203+2$ \\
96.25 \\
79.05 \\
66.27 \\
33.57 \\
20.66 \\
14.14 \\
10.34 \\
7.915 \\
6.263 \\
5.082 \\
4.205 \\
3.534 \\
3.009 \\
2.589 \\
2.249 \\
1.967 \\
1.733 \\
1.535 \\
1.367 \\
1.098 \\
$6.712-1$ \\
$4.337-1$ \\
$2.906-1$ \\
$1.998-1$ \\
$1.399-1$ \\
$9.941-2$ \\
$7.142-2$ \\
\\
\end{tabular}

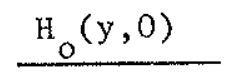

$\underline{G_{0}(y)}$

$6.271+5$

$2.910+4$

$1.155+4$

$4.581+3$

$2.677+3$

$1.817+3$

1. $348+3$

$5.335+2$

$3.096+2$

$2.102+2$

$1.555+2$

1. $214+2$

98.37

81.94

69.69

60.25

34.15

22.59

16.26

12.34

9.713

7.850

6.474

5.424

4.602

3.947

3.414

2.975

2.610

2.302

2.040

1.816

1.454

8.771-1

5.557-1

$3.642-1$

2. $445-1$

$1.672-1$

1.160-1

8.145-2
973

213.6

133.6

83.49

63.29

51.92

44.50

27.36

20.45

16.57

14.03

12.22

10.85

9.777

8.905

8.182

5.832

4.517

3.663

3.059

2.607

2.225

1.973

1.742

1.549

1.386

1.246

1.126

1.020

9.280-1

8.465-1

7.740-1

$6.514-1$

4.359-1

3.004-1

2.113-1

1. 508- 1

1.089-1

7.926-2

5.811-2 


\begin{tabular}{|c|c|}
\hline$y$ & $\mathrm{~K}_{1 / 3}(\mathrm{y})$ \\
\hline 3.00 & $3.531-2$ \\
\hline 3.25 & $2.645-2$ \\
\hline 3.50 & 1. $988-2$ \\
\hline 3.75 & $1.497-2$ \\
\hline 4.00 & $1.130-2$ \\
\hline 4.25 & $8.545-3$ \\
\hline 4.50 & $6.472-3$ \\
\hline 4.75 & $4.909-4$ \\
\hline 5.00 & $3.729-3$ \\
\hline 5.50 & $2.159-3$ \\
\hline 6.00 & 1. $255-3$ \\
\hline 6.50 & $7.317-4$ \\
\hline 7.00 & $4.280-4$ \\
\hline 7.50 & $2.509-4$ \\
\hline 8.00 & $1.474-4$ \\
\hline 8.50 & $8.679-5$ \\
\hline 9.00 & $5.118-5$ \\
\hline 9.50 & $3.023-5$ \\
\hline 10.00 & $1.787-5$ \\
\hline
\end{tabular}

\begin{tabular}{l}
$\mathrm{K}_{2 / 3}(\mathrm{y})$ \\
\hline $3.706-2$ \\
$2.767-2$ \\
$2.073-2$ \\
$1.558-2$ \\
$1.173-2$ \\
$8.853-3$ \\
$6.693-3$ \\
$5.069-3$ \\
$3.844-3$ \\
$2.220-3$ \\
$1.287-3$ \\
$7.495-4$ \\
$4.376-4$ \\
$2.562-4$ \\
$1.504-4$ \\
$8.842-5$ \\
$5.209-5$ \\
$3.073-5$ \\
$1.816-5$
\end{tabular}

\begin{tabular}{l}
$\mathrm{K}_{5 / 3}(\mathrm{y})$ \\
\hline $5.178-2$ \\
$3.781-2$ \\
$2.778-2$ \\
$2.051-2$ \\
$1.521-2$ \\
$1.132-2$ \\
$8.455-3$ \\
$6.332-3$ \\
$4.754-3$ \\
$2.697-3$ \\
$1.541-3$ \\
$8.855-4$ \\
$5.113-4$ \\
$2.965-4$ \\
$1.725-4$ \\
$1.007-4$ \\
$5.890-5$ \\
$3.454-5$ \\
$2.030-5$
\end{tabular}

\begin{tabular}{l}
$H_{0}(y, 0)$ \\
\hline $5.772-2$ \\
$4.123-2$ \\
$2.964-2$ \\
$2.144-2$ \\
$1.558-2$ \\
$1.138-2$ \\
$8.338-3$ \\
$6.132-3$ \\
$4.523-3$ \\
$2.481-3$ \\
$1.373-3$ \\
$7.659-4$ \\
$4.299-4$ \\
$2.426-4$ \\
$1.376-4$ \\
$7.837-5$ \\
$4.480-5$ \\
$2.569-5$ \\
$1.478-5$
\end{tabular}

$G_{0}(y)$

4.286-2

3. $175-2$

2. $362-2$

1.764-2

1.321-2

9.915-3

7.461-3

5.626-3

4.250-3

$2.436-3$

1.404-3

8.131-4

$3.842-4$

2. $755-4$

1. $611-4$

9.439-5

5. 543-5

3.262-5

1.922-5 


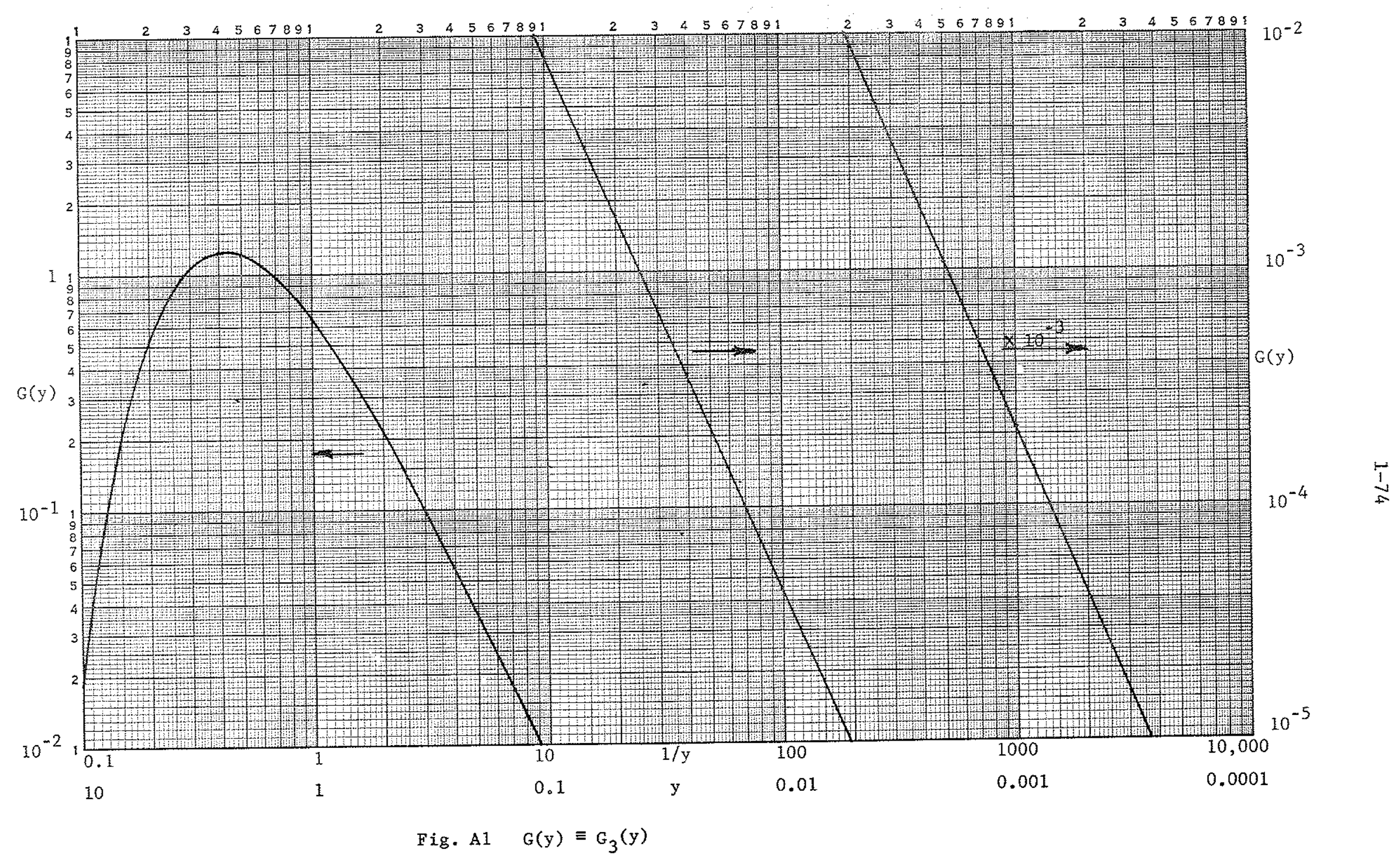


APPENDIX B

Outline of Storage Ring Particle Dynamics

Properties of the synchrotron radiation source depend upon the angular distribution of the radiation process and on the spacial and angular distribution of the electron beam. The radiation angles are a function of $\lambda / \lambda_{c}$ and $Y$ (Section I.2.) and can be controlled to the extent that those para meters can be chosen. Electron beam distributions are a function of the storage ring design and, in an alternating-gradient rino, will vary with circumferential position. In this brief note only the properties of separated-function alternating gradient stage rings will be considered. The separated function ring has dipoles (negligible fi-ld gradient) which bend the electron beam a total of $2 \pi$ and quadrupoles (constant transverse gradient magnets) which supply the restoring forces that keep the beam "focussed". A quadrupole has zero field on iis axis and exerts converging, or focussing, forces in one transverse plane and diverging, or defocussing, forces in the normal transverse plane. (Ref. 15, Chap. 6.) A sequence of quadrupoles is normally arranged with focussing and defocussing directions alternating. Such a sequence will then be net focussing over a rather wide range of parameters.

The central orbit of a storage ring is that orbit which will be repetin tively traced by a particle of design energy launched precisely on that orbit. The central orbit is constructed by joining circular ares in the bending magnets to segments of straight lines in the quadrupoles and field free sections ("straight-sections".) Solution of the geometrical problem is done by requiring that the central orbit close on itself. Two examples of very different magnet lattices are shown in Fig. BI with the central orbit indicated by a dashed line.* In order to ensure that particles will remain indefinitely in the neighborhood of the central orbit when subjected to disturbances and imperfections, strong restoring forces are applied by the quadrupoles inserted in the lattice.

The energy of an electron is continually being changed (in small in.crements) by photon radiation and by the acceleration system of the ring. * The examples of this section were selected from a series of orbit studies done by Renate Chasman. 
An electron with energy differing from that of the central orbit will then have a different radius of curvature in the bending magnets $\rho=33.35 \mathrm{E} / \mathrm{B}$ (Eq. 16) and will travel on a larger, or smaller, circumference. A closed equilibrium orbit exists for these off-energy particles but the field of the quadrupole is no longer zero off-axis. A recursion function can be written for the motion of an electron through the bending magnets and quadrupoles and this function can be solved, in principle, for the closed equilibrium orbit. Solution is best done numerically by one of the numerous programs used by accelerator designers. If $s$ is the coordinate along the trajectory a monentum function $x_{p}(s)$ is defined by

$$
\Delta x=X_{p}(s) \frac{\Delta E}{\mathrm{E}_{0}}
$$

where $\Delta x$ is the local setover of the equilibrium orbit for energy change $\Delta E$ from the central orbit energy $\mathrm{E}_{\mathrm{O}}{ }^{16}$ (The momentum function is also referred to as $\alpha_{p}$ or $\left.\eta_{\bullet}\right)$ In Fig. $\mathrm{B} 2$ the $\mathrm{X}_{\mathrm{p}}$ is always finite. For this type of FODO lattice the off-axis equilibrium orbit is adjacent to the central orbit but has "wiggle-motion" which is correlated with the focussing periodicity. The triplet lattice functions shown in B3 illustrate the effects of an achromatic bend design. $x_{p}$ is finite in the bending magnet sectors but is approximately zero in the insertions between sectors.

The radio frequency accelerating waveform has a phase focussing action and the radiation damps the longitudinal motion. This results in the electron beam being compressed into short bunches with energy distribution $\sigma_{p}$ which has a minimum value determined by the quantum fluctuations of the photon radiation. (The observed value of $\sigma_{p}$ will be increased by intrabeam scattering and by instabilities.) Thus the ensemble of equilibrium orbits in a ring will be a band of width

$$
\Delta x(s)= \pm X_{p}(s) \sigma_{p}
$$

about the central orbit. 
If an electron is displaced in position and/or angle from its equilibrium orbit it will execute "betatron" oscillations about that orbit. Let $s$ be the distance along the equilibrium orbit and $x$ be the radial and $y$ the vertical displacement from the equilibrium orbit of the electron. A prime will denote the derivative with respect to s. The transverse motion can be described by the phase-amplitude solution

$$
\begin{aligned}
& x=A_{x} \beta_{x}^{\frac{1}{2}} \sin \left(\int \frac{d s}{\beta_{x}}+\delta_{x}\right) \\
& y=A_{y} \beta_{y}^{\frac{1}{2}} \sin \left(\int \frac{d s}{\beta_{y}}+\delta_{y}\right)
\end{aligned}
$$

Constants $A$ and $\delta$ are determined by the initial conditions. $\beta$ is an amplitude function which can be calculated from the transformation matrix of the ring lattice. ${ }^{16}$ Figures $B 2$ and $B 3$ show $\beta$ as a function of $s$ for the two examples. If $\beta$ is constant the equations are those of the simple harmonic oscillator with wavelength given by $\beta=\lambda / 2 \pi$. In an alternating gradient ring the motion is that of an oscillator in which the amplitude multiplier varies while the phase advance speeds up and slows down. One wavelength of betatron oscillation is completed when

$$
\int_{s_{1}}^{s_{2}} \frac{d s}{\beta}=2 \pi
$$

A very important number is the $v$ value, the number of oscillation cycles per turn

$$
\nu=\frac{1}{2 \pi} \int_{s}^{s+c} \frac{d s}{\beta}, \quad c=\text { circumference }
$$

In order to avoid resonance effects $v$ must not be a small rational fraction. One can then visualize the transverse motion as a sinusoid precessing around the ring and superimposed on it a wiggle motion fixed to the magnet lattice and related to $\beta^{\frac{1}{2}}$. If there is a beam circulating with a large number of particles distributed in phase and amplitude the envelope of the betatron oscillations is proportional to $\beta^{\frac{3}{2}}$ vs s. 
Define two other variables,

$$
\begin{aligned}
& \alpha=-\beta^{\prime} / 2 \\
& \gamma=\left(1+\alpha^{2}\right) / \beta
\end{aligned}
$$

Then $\alpha, \beta, \gamma$ are coefficients of the transfer matrices ${ }^{16}$ which can be used to transform the position and angle of the betatron motion at any azimuth of the ring to any other azimuth. They are also the coefficients of the courant-Snyder invariant

$$
\mathrm{E}_{\mathrm{x}}=\frac{1}{\beta_{\mathrm{x}}}\left[\mathrm{x}^{2}+\left(\alpha_{\mathrm{x}} \mathrm{x}+\beta_{\mathrm{x}} \mathrm{x}^{\prime}\right)^{2}\right]=\gamma_{\mathrm{x}} \mathrm{x}^{2}+2 \alpha_{\mathrm{x}} \mathrm{xx} \mathrm{x}^{\prime}+\beta_{\mathrm{x}} \mathrm{x}^{\prime 2}
$$

(and similarly for $y$, in uncoupled motion). The right-hand side is the equation of an ellipse in $x$ and $x^{\prime}$. Since $\alpha, \beta, \gamma$ are functions of $s$, the shape and inclination of the ellipse in the phase space $x$, $x^{\prime}$ will vary with $s$ but the area will remain invariant. A particle with displacement and angle $x_{1}$, $x_{1}^{\prime}$ at $s_{0}$ will lie on ellipse

$$
E_{1}=\gamma\left(s_{0}\right) x_{1}^{2}+2 \alpha\left(s_{0}\right) x_{1} x_{1}^{\prime}+\beta\left(s_{0}\right) x_{1}^{\prime 2}
$$

and will transverse the path in phase space defined by $E_{1}$ as it goes around the ring. If an ensemble of particles lies within boundary $E$ at $s$ it will lie within $\mathrm{E}$ everywhere around the ring. At any $s$ the maximum $x$ of any particle will be

$$
\hat{x}=[\beta(s) E]^{\frac{1}{2}}
$$

and the maximum angle

$$
\hat{x}^{\prime}=\left[\gamma(s) E^{\prime}\right]^{\frac{1}{2}}
$$

Enter the ring at a $\beta_{\max }$ or $\beta_{\min }$ where $\alpha=0$ and consequent $1 \mathrm{y} \beta=1 / \gamma$. The ellipse axes lie on $x^{\prime}$ and $x^{\prime}$ and the area of the ellipse is

$$
\pi \mathrm{xX} \mathrm{x}^{\prime}=\pi \mathrm{E}
$$

$E$ is the emittance of the beam. There is an $E_{x}$ and an $E_{y}$. 
Thus the largest beam size will occur at a $\beta_{\max }$ and the largest divergence at a $\beta_{\mathrm{min}}$ :

$$
\hat{x}=\left[E_{x} \hat{\beta}_{x}\right]^{\frac{3}{2}} ; \quad \hat{x}^{\prime}=\left[E_{x} / \beta_{x \min }\right]^{\frac{1}{2}}
$$

Although the size-angle product of the beam can be considered crudely constant, this is strictly true only at a $\beta_{\max }$ or a $\beta_{\min }$. Elsewhere the ellipse containing the beam is inclined and the $\hat{x} \hat{x}^{\prime}$ product will be larger than $\mathrm{E}$ 。

The foregoing relations are valid for a conservative system or for a radiating system in quasi-equilibrium.

An electron position $x_{0}, x_{0}^{\prime}$ at $s_{0}$ will be at $s_{1} 16$

$$
\left|\begin{array}{l|}
x_{1} \\
x_{1}^{\prime}
\end{array}\right|\left|\begin{array}{ll}
a_{11} & a_{12} \\
a_{21} & a_{22}
\end{array}\right|\left|\begin{array}{c}
x_{0} \\
x_{p}^{\prime}
\end{array}\right|
$$

A focussing quadrupole of length $\ell$ and gradient $G=\mathrm{dB} / \mathrm{dx}$ operating on a particle with magnetic rigity $B \rho$ has transformation matrix;

$\left|\begin{array}{ll}\cos \ell \sqrt{\mathrm{K}} & \frac{1}{\sqrt{\mathrm{K}}} \sin \ell \sqrt{\mathrm{K}} \\ -\sqrt{\mathrm{K}} \sin \ell \sqrt{\mathrm{K}} & \cos \ell \sqrt{\mathrm{K}}\end{array}\right|$

with $\quad K=G / B \rho$

and a defocussing quadrupole has transformation matrix;

$\cosh \ell \sqrt{k}$

$\frac{1}{\sqrt{K}} \sinh \ell \sqrt{K}$

$\sqrt{\mathrm{K}} \sinh \ell \sqrt{\mathrm{K}}$

$\cosh \ell \sqrt{K}$ 
The same transformations apply in $y, y^{\prime}$ with focussing and defocussing interchanged, i.e. a focussing quadrupole in the $x$ coordinate is defocussing in the $y$ coordinate and vice versa. A non-focussing section of length 2 has transformation matrix:

$$
\left|\begin{array}{ll}
1 & \ell \\
0 & 1
\end{array}\right|
$$

(Bending magnets have small focussing effects, especially at their ends, but these effects can be disregarded when examining a source. The axis $s$ in a bending magnet is curvilinear with radius $\rho_{0}$ ) Transformation through multiple elements is then a matrix obtained by multiplying the matrices of the elements in succession.

An ellipse

$$
\gamma_{0} x^{2}+2 \alpha_{0} x x^{\prime}+\beta_{0} x^{\prime 2}=E
$$

can be transformed from $s_{0}$ to $s_{1}$ by

$$
\left|\begin{array}{l}
\alpha_{1} \\
\beta_{1} \\
\gamma_{1}
\end{array}\right|=\left|\begin{array}{ccc}
a_{11} a_{22}{ }^{+a_{12} a_{21}} & -a_{11} a_{21} & -a_{12} a_{22} \\
-2 a_{11} a_{12} & a_{11}^{2} & a_{12}^{2} \\
-2 a_{21} a_{22} & a_{21}^{2} & a_{22}^{2}
\end{array}\right|\left|\begin{array}{c}
\alpha_{0} \\
\beta_{0} \\
\gamma_{0}
\end{array}\right|
$$

in which the $a_{i j}$ are the elements of the $x, x^{\prime}$ transformation matrix from $s_{0}$ to $s_{1}$. The determinant of this matrix must be 1 so $a_{11} a_{22}-a_{12} a_{21}=1$. If. there is no focussing this becomes;

$$
\left|\begin{array}{l}
\alpha_{1} \\
\beta_{1} \\
\gamma_{1}
\end{array}\right| \quad\left|\begin{array}{ccc}
1 & 0 & -l \\
-2 \ell & 1 & \ell^{2} \\
0 & 0 & 1
\end{array}\right|\left|\begin{array}{l}
\alpha_{0} \\
\beta_{0} \\
\gamma_{0}
\end{array}\right|
$$

or

$$
\begin{aligned}
& \alpha_{1}=\alpha_{0}-l \gamma_{0} \\
& \beta_{1}=2 l \alpha_{0}+\beta_{0}+l^{2} \gamma_{0} \\
& \gamma_{1}=\gamma_{0}
\end{aligned}
$$


If initial conditions of $\alpha, \beta, \gamma$ are known from design tables or from curves such as Figs。B2, B3 then coefficients are readily determined at distance $\ell$ along $s$ 。 An important special case begins at the center of an insertion where $\beta_{0}$ is a minimum, simplify to

$$
\alpha_{0}=-\beta^{\prime} / 2=0 \text { and } \gamma_{0}=\left(1+\alpha^{2}\right) / \beta_{0}=1 / \beta_{0} \text { Then the equations }^{2} \text { The }
$$

$$
\begin{aligned}
& \alpha_{1}=-l \gamma_{0}=-\ell / \beta_{0} \\
& \beta_{1}=\beta_{0}+\ell^{2} \gamma_{0}=\beta_{0}+\ell^{2} / \beta_{0} \\
& \gamma_{1}=\gamma_{0}=1 / \beta_{0}
\end{aligned}
$$

The electron beam configuration of a source is determined from the ring parameters at the azimuth, or $s$, of the source. It is usually adequate to assume that the vertical equilibrium orbits lie in the median plane and that there is zero vertical momentum function. The vertical emittance and vertical amplitude functions at tue crosen azimuth then give the vertical size and angular distribution.

Radial distribution of the equilibrium orbits is given by $\sigma_{p} x_{p}$ with $\sigma_{p}$ the variance of energy spread $\Delta E / E$. Betatron oscillations occur about these equilibrium orbits with variance $\sigma_{x}$ obtained from tne emittance and horizontal amplitude functions. The energy oscillations are at a low frequency, the betatxon oscillations are at a high frequency, and the two are uncorrelated. The horizontal. width variance is obtained by adding in quadrature

$$
\sigma_{h}^{2}=\left(\sigma_{p} x_{p}\right)^{2}+\sigma_{x}^{2}
$$

It is often sufficient to assume that $x_{p}$ is constant at tne value at the center of the source. 


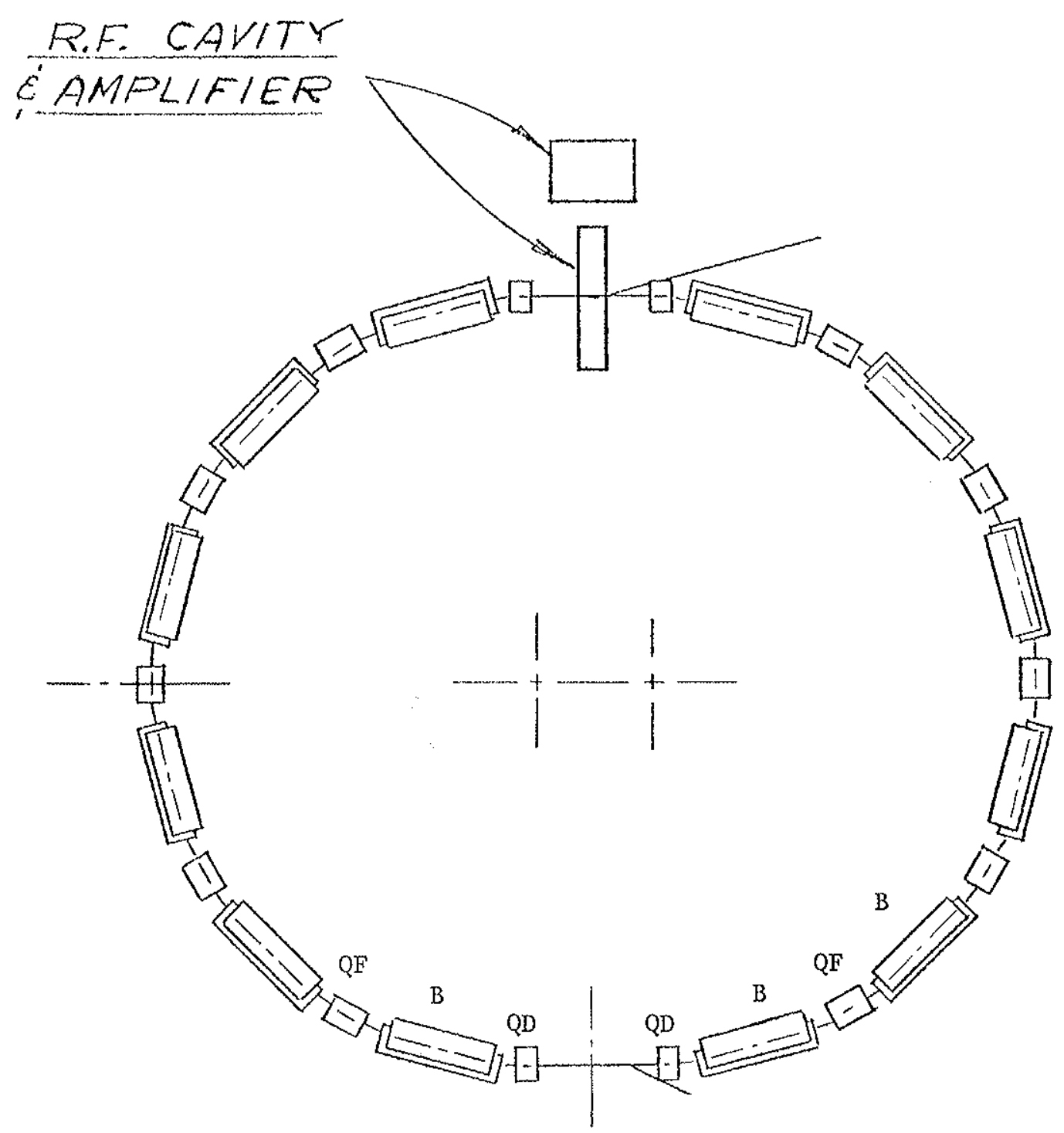

Fig. Bla. Ring with FODO lattice. Q-quadrupole, B-bending magnet. 


\section{$1-83$}

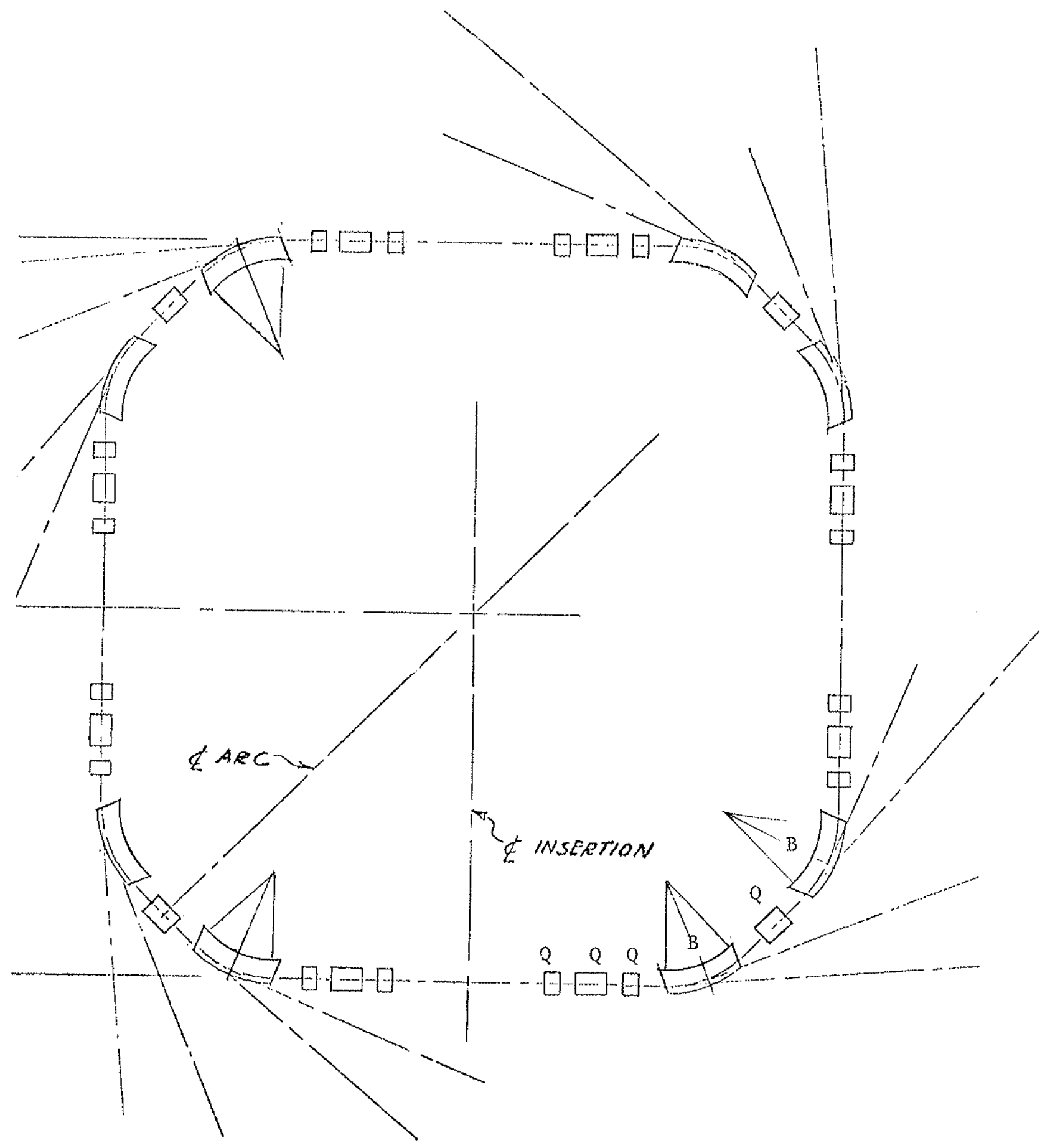

Fig. Blb. Ring with triplett lattice. Q-quadrupole, B-bending magnet. 


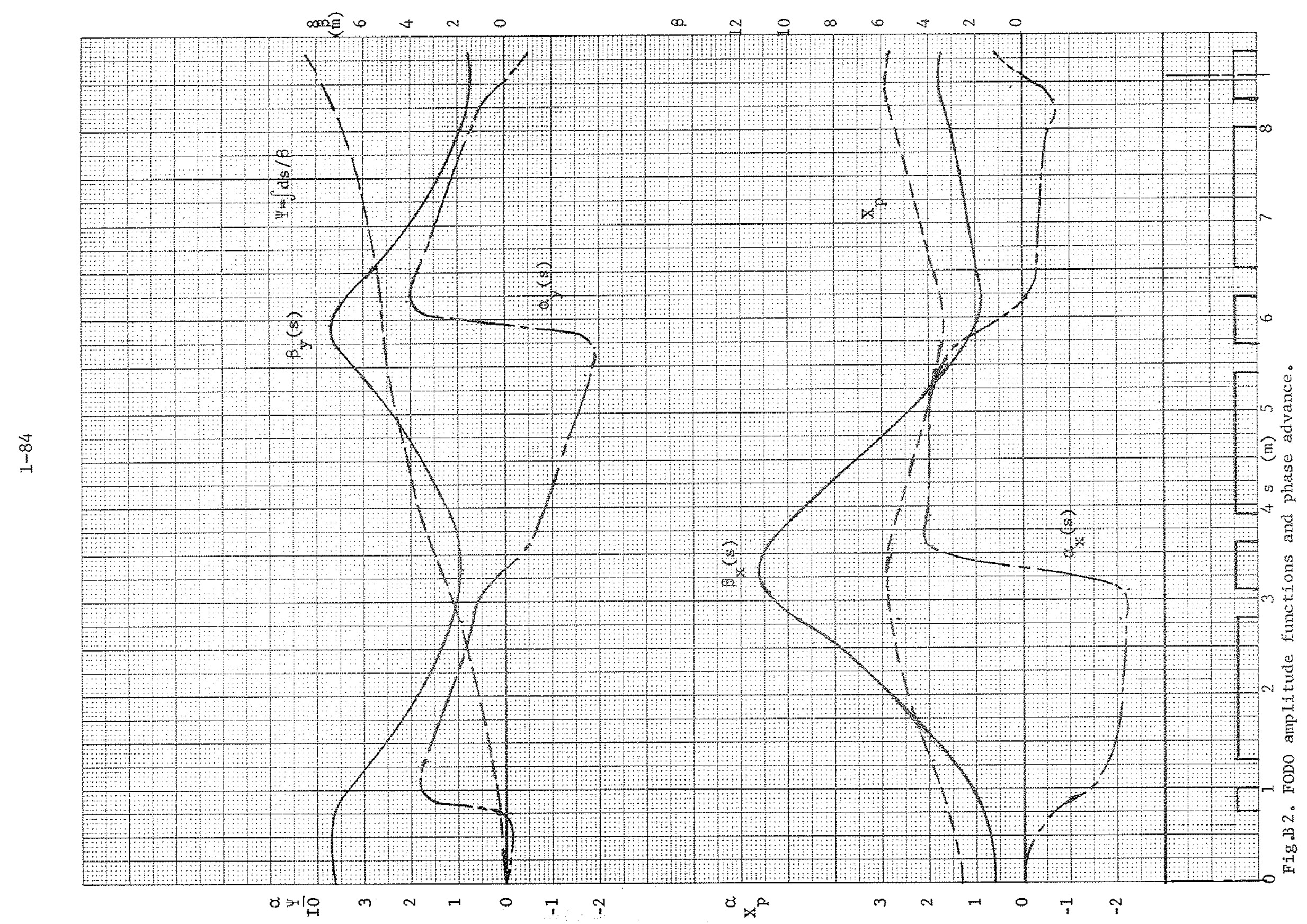




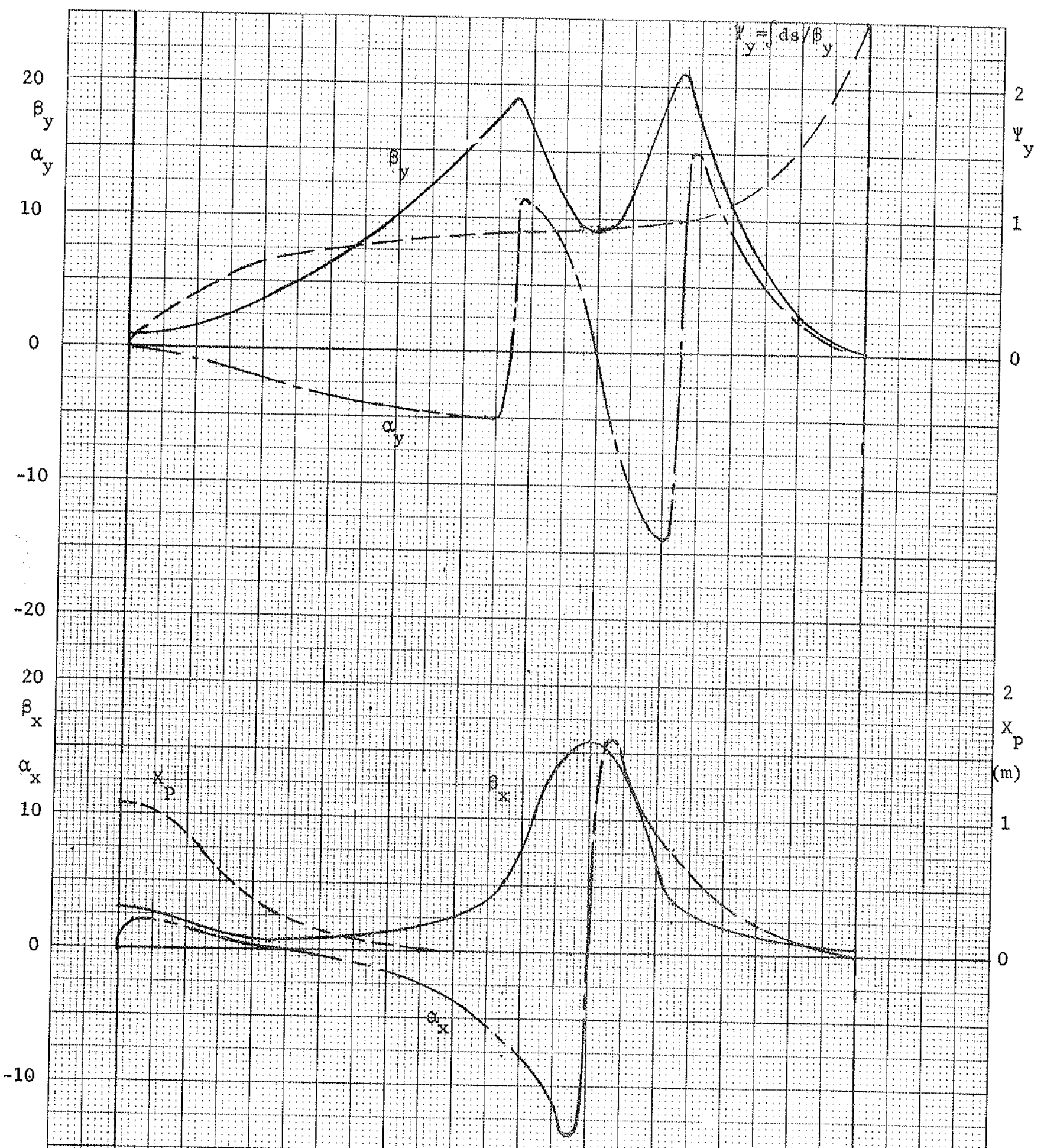

$-20$

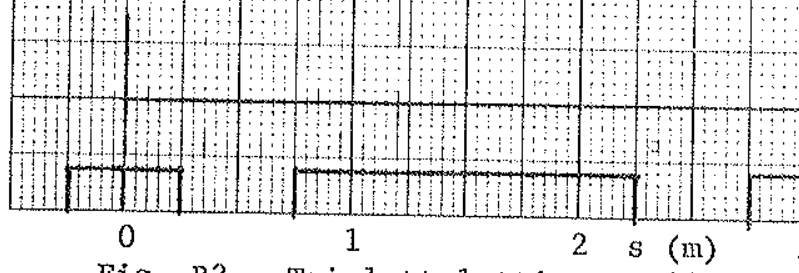

Fig. B3. Triplett lattice amplitude functions and phase advance. 


\section{APPENDTX C}

Exponential Integrating Eunction ef $(a, Y)$

A function of $z$
\[ \int_{0}^{z} 1 \frac{1}{\sqrt{b^{2}+c^{2} z^{2}}} e^{-\frac{1}{2} \frac{y^{2}}{\left(b^{2}+c^{2} z^{2}\right)} d z} \]

in which $\mathrm{b}, \mathrm{c}$, and $\mathrm{y}$ are constants can be transformed by

$$
\cos t=\frac{b}{\sqrt{b^{2}+c^{2} z^{2}}}, \quad \tan t=\frac{c z}{b}, d z=\frac{b}{c} \sec ^{2} t d t
$$

to $\quad \frac{1}{c} \int_{0}^{\tan ^{-1} \frac{c z}{b}} \frac{1}{\cos t} e^{-\frac{y^{2}}{2 b^{2}} \cos ^{2} t} d t$

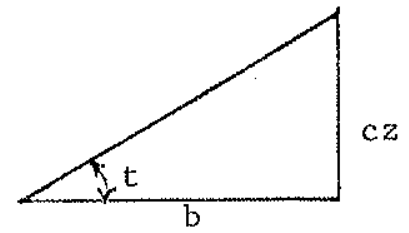

Define ef $(a, Y)=\int_{0}^{Y} \frac{1}{\cos t} e^{-\frac{a^{2}}{2} \cos ^{2} t} d t \quad Y<\frac{\pi}{2}$

with, for $f(z), a=y / b, \tan Y=c z_{1} / b$

To normalize, Ref. 11. No. 492,

$$
\begin{aligned}
& \int_{0}^{Y} \int_{0}^{\infty} \frac{1}{\cos t} e^{-\frac{a^{2}}{2} \cos ^{2} t} d t d a=\sqrt{ } \frac{\pi}{2} \int_{0}^{Y} \frac{1}{\cos ^{2} t} d t=\sqrt{ } \frac{\pi}{2} \tan Y \\
& \int_{-\infty}^{\infty} e f(a, Y) d a=\sqrt{2 \pi} \tan Y
\end{aligned}
$$

At $a=0$

$$
\text { ef }(0, Y)=\int_{0}^{Y} \frac{d t}{\cos t}=\frac{1}{2} \ln \left(\frac{1+\sin Y}{1-\sin Y}\right) \quad \text { (Ref. 11. No. 288) }
$$

If $Y<0.1 \cos t \approx 1$

$$
\text { and } \text { ef }(a, Y)=\int_{0}^{Y} e^{-\frac{a^{2}}{2}} d t=y e^{-\frac{a^{2}}{2}}
$$




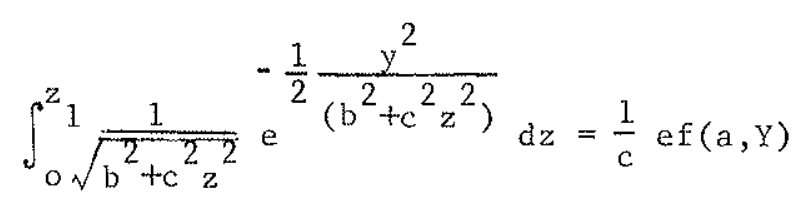

with $\quad a=y / b, \quad \tan y=c z_{1} / b$

Graphs of ef $(a, Y)$ and ef $(O, Y)$ are $F$ igs. $C 1$ and $C 2$. A short table of the function is included as Table $\mathrm{CI}$. This table was kindly programmed and run by Kurt Jellet. 
IABLE CI ef $(a, Y)$

Mantissa following table entry denotes power of ten multiplier.

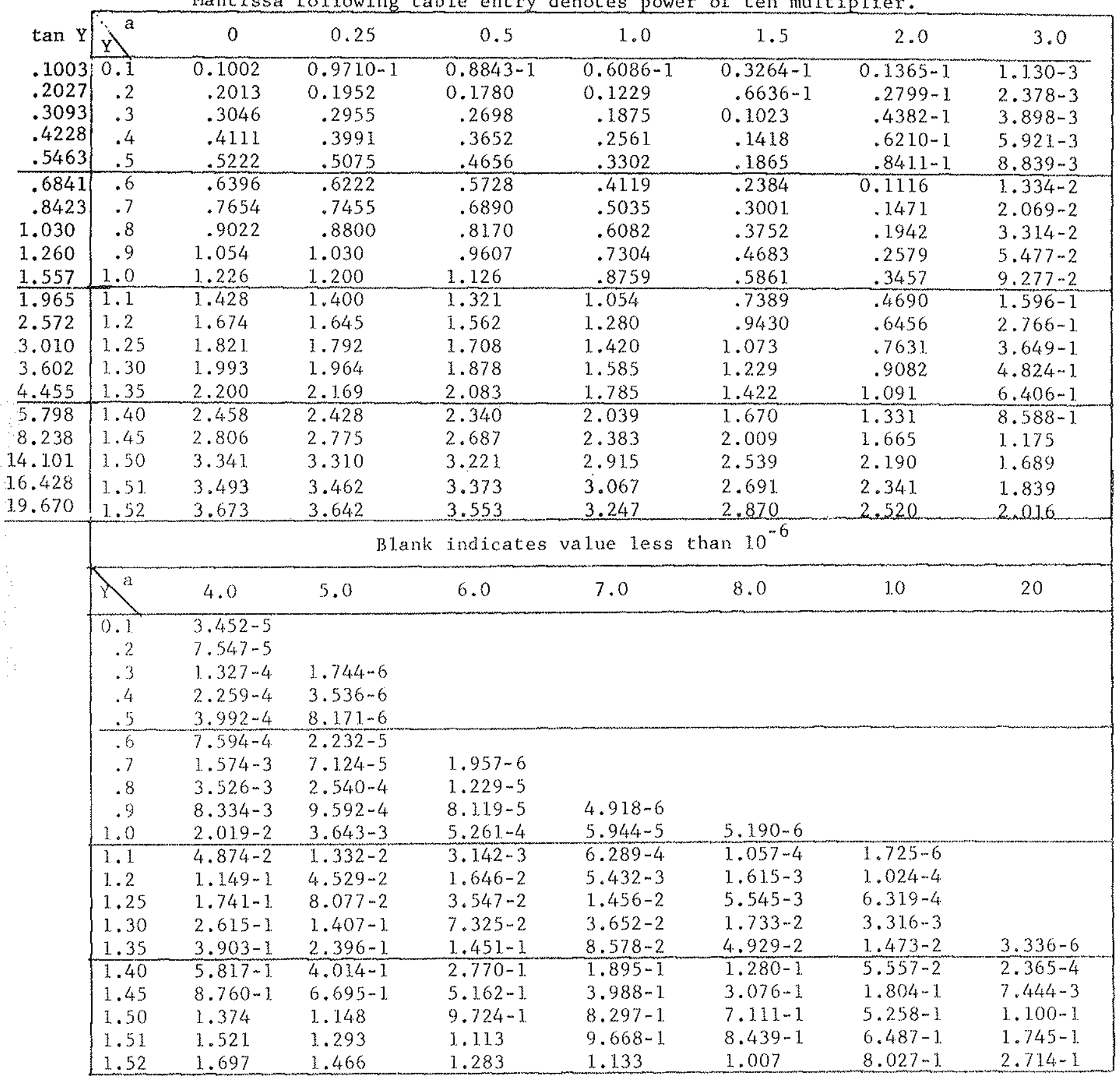


3

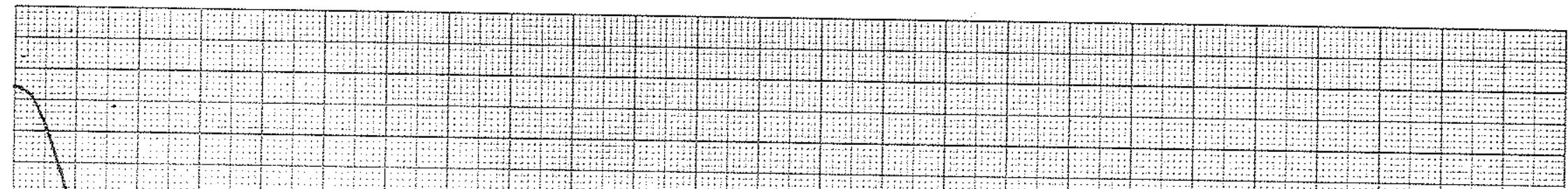

2<smiles>CC1CCCC1</smiles>

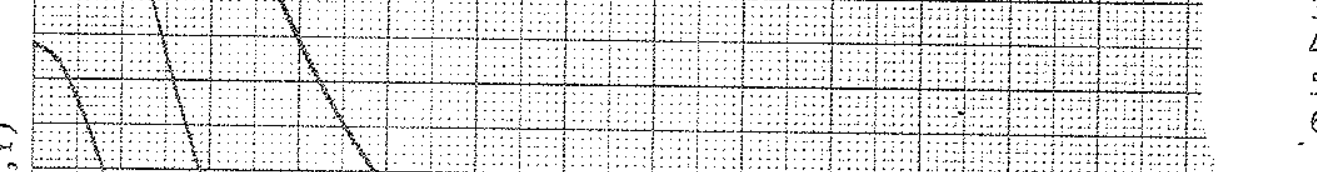

Curve

$\frac{1}{2}$

4

6

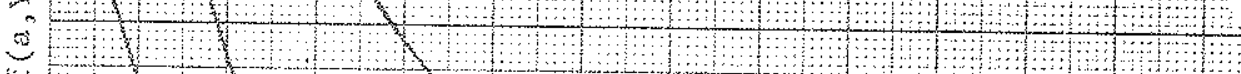

$\frac{x}{0.1}$

0.5
1.0

1.2

1.4

3.5

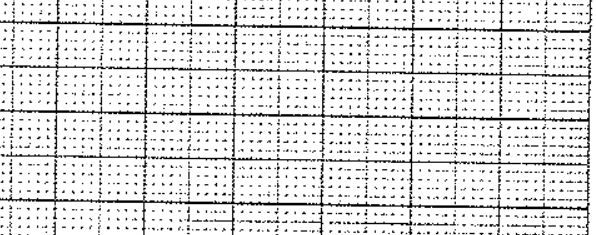

1

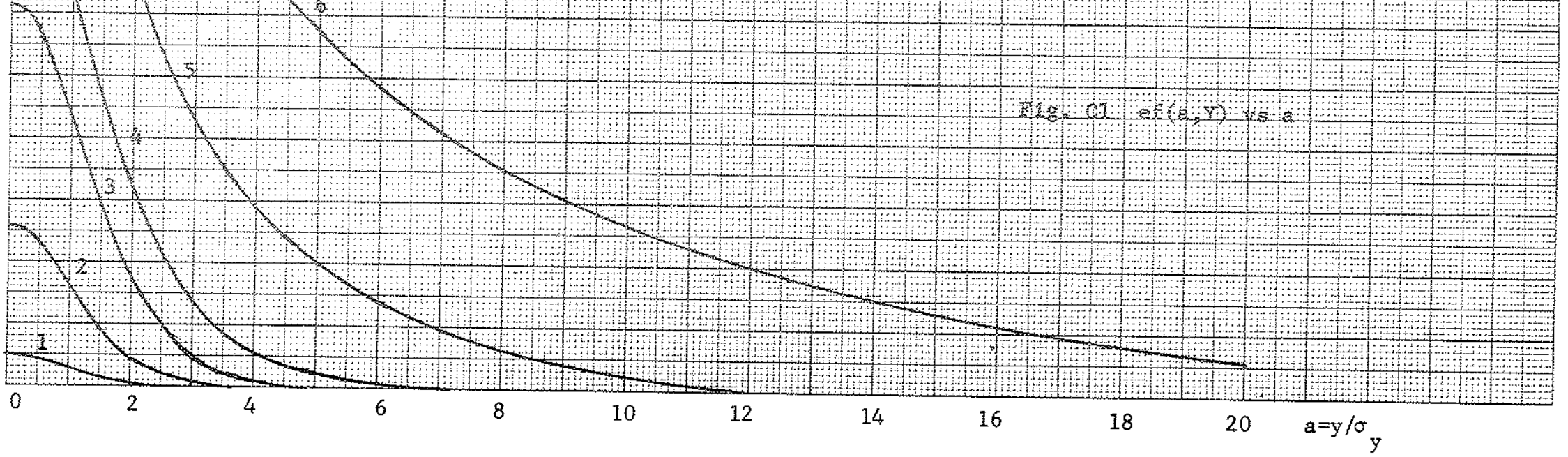




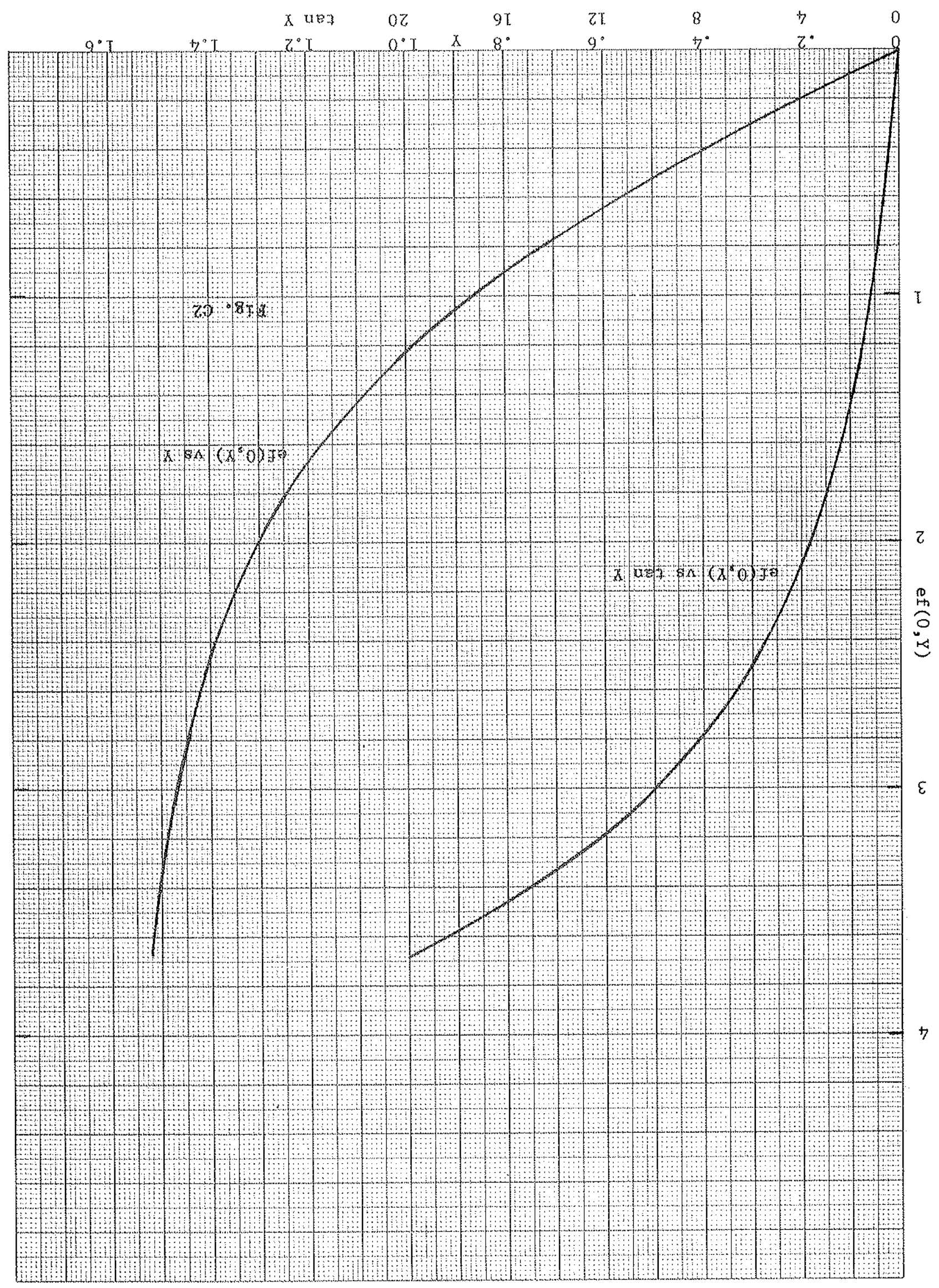




\title{
The Synchrotron Radiation Source
}

\author{
G。 K. Green
}

\section{Brookhaven National Laboratory * \\ Accelerator Department \\ Upton, New York}

The synchrotron light source is described by combining the properties of the radiation and the configuration of the electron beam. Most of the radiation is polarized parallel to the orbital plane and this radiation is most efficiently transmitted in vertical dispersion. The $\|$ component will be treated in this note in order to simplify the equations.

Radiation functions $H_{2}(y), G_{1}(y)$, yo and $f$ are shown in $F i g, 1$ vs $y$. The number of photons in interval $\Delta \lambda=\mathrm{k} \lambda$ per second is

$$
\begin{aligned}
& N_{k}(\lambda)=1.256 \times 10^{16} \mathrm{kIOVG}_{1}\left(\lambda_{\mathrm{c}} / \lambda\right) \text { in a11 } \psi \\
& \mathrm{N}_{\mathrm{k}}(0, \lambda)=3.461 \times 10^{15} \mathrm{kI \theta y}^{2} \mathrm{H}_{2}\left(\lambda_{\mathrm{c}} / \lambda\right) \text { per rad } \psi \text { at } \psi=0
\end{aligned}
$$

with I in amps, orbit angle $\theta$ in radians and $\gamma=1957 \mathrm{E}(\mathrm{GeV}) .^{\dagger} \mathrm{N}_{\mathrm{k}}(0, \lambda)$ is al1 $\|$ at $\psi=0$ and $F$ is the fraction of total photons in $\Delta \lambda$ that are $\|$ polarized. A good approximation up to $\lambda \approx 100 \lambda_{c}$ of the angular distribution in $\psi$ is given by

$$
N_{k}(\psi, \lambda)=N_{k}(0, \lambda) \exp \left(-\psi^{2} / 2 \sigma^{2}\right)
$$

with

$$
\gamma=1.45 \mathrm{EG}_{1} / \mathrm{H}_{2} \quad \text { radians }
$$

The electron beam is described by the variance of its size and angular distributions $\sigma_{x}, \sigma_{x}$, radial and $\sigma_{y}, \sigma_{y}$, vertical, of which $\sigma_{x}$ will include the spread due to monentum dispersion. These variances are a function of azimuthal variable s and are given by the ring emittances and amplitude

\footnotetext{
Work performed under the auspices of the U.S. Energy Research and Development Administration 
functions. The electron bean is shown at $s=0$ (Fig. 2) in $x, x^{\prime}$ and $y, y^{\prime}$ phase space at a waist or $\beta$ minimum. As we move to $s$ the bean figure will transform to the dashed ellipse and the radiation from a ds at $s$ will increase the angles, but not the size. The radiation figure can then be transformed back to $s=0$. If all the contributions for $\pm s$ are transformed to $s=0$ their sum is a planar optical source which can be transformed into the desired image space. Using some simplifying approximations the axial distributions in this source are:

$$
\mathrm{dN}_{k}(y)=\mathrm{U} \frac{\gamma^{2} \mathrm{H}_{2}}{\mathrm{D}} \mathrm{dy} \text { ef }(\mathrm{a}, \mathrm{Y}), \mathrm{a}=\mathrm{y} / \sigma_{\mathrm{y}}, \tan \mathrm{Y}=\sigma \mathrm{s} / \sigma_{\mathrm{y}}
$$

$U$ is a numerical factor containing $k$ and $T$. ef is an integrating function approximated by $Y \exp \left(-\mathrm{a}^{2} / 2\right)$ for $Y$ less than 0.2 or 0.3 .

$$
\begin{aligned}
& \mathrm{dN}_{k^{\prime}}\left(\mathrm{y}^{\prime}\right)=\mathrm{U}_{1} \frac{\gamma^{2} \mathrm{H}_{2}}{\rho} \mathrm{dy^{ \prime }} \frac{\sigma \mathrm{s}}{\sigma_{\mathrm{Y}^{\prime}}} \exp \left(-\mathrm{y}^{2} / 2 \sigma_{Y^{\prime}}^{2}\right) ; \sigma_{Y^{\prime}}^{2}=\sigma_{\mathrm{y}^{\prime}}^{2}+\sigma^{2} \\
& \mathrm{dN}_{\mathrm{k}}(\mathrm{x})=\mathrm{U}_{2} \gamma \mathrm{fG} \mathrm{G}_{1} \frac{\mathrm{dx}}{\sigma_{\mathrm{x}}} \exp \left[-\left(\mathrm{x}-\mathrm{s}^{2} / 2 \rho\right)^{2} / 2 \sigma_{\mathrm{x}}^{2}\right] ; \sigma_{\mathrm{X}}^{2}=\sigma_{\mathrm{x}}^{2}+\mathrm{s}^{2} \sigma^{2} \\
& \mathrm{dN}_{\mathrm{k}}\left(\mathrm{x}^{\prime}\right)=\mathrm{U}_{2} \quad \gamma \in \mathrm{G}_{1} \mathrm{dx} \mathrm{x}^{\prime}
\end{aligned}
$$

Each of the above distributions contains the integral of the orthogonal coordinate.

The central phase space density is

$$
\begin{aligned}
& d^{2} N_{k y}(0,0)=U_{3} \frac{\gamma^{2} H_{2}}{\rho} \frac{d y d y^{\prime}}{\sigma_{y^{\prime}}} \sinh ^{-1}\left(\frac{s \sigma \sigma y^{\prime}}{\sigma_{y}^{\sigma} y^{\prime}}\right) \\
& d^{2} N_{k x}(0,0)=U_{4} \gamma f G_{1} d x d x^{\prime} / \sigma_{x}
\end{aligned}
$$

$\mathrm{X}$-ray sources will usually subtend a small orbit angle $\theta$. To illustrate the varfations three typical examples will be tabulated, a $4 \mathrm{GeV}$ colliding beam ring bending magnet source, a $2 \mathrm{GeV}$ low emittance ring insertion wiggler source, and a $2.5 \mathrm{GeV}$ low emittance ring bending magnet source; all for $\theta \pm 1$ mrad and per unit current. 
A

$$
4 \mathrm{GeV} \text { arc }
$$

$\gamma$

$\lambda_{c}(\AA)$

$\rho(m)$

$\mathrm{s}(\mathrm{m})$

$\sigma_{y}(\mathrm{~mm})$

$\sigma_{y}($ mrad $)$

$\sigma_{\mathrm{x}}(\mathrm{mm})$

$\sigma(\mathrm{mrad})$
7830

1.1

12.7

0.013

0.23

0.04

1.4

0.073
B

$2 \mathrm{GeV}$ insertion

3910

1.1

1.67

0.0017

0.006

0.025

0.1

0.146
C

$2.5 \mathrm{GeV}$ arc

4890

2.0

8.1

0.0082 per mrad $\theta$

0.04

0.017

0.3

0.117 at $y=1$

The short source length and $\sigma_{y}{ }^{\prime}<\sigma$ permit further approximations, $\sigma_{Y}=\sigma$, $\sigma_{X}=\sigma_{x}$ and :

$$
\begin{aligned}
& \mathrm{dN}_{k}(y)=\left(U_{1} \gamma^{2} H_{2} d y\right)\left(\sigma s / \sigma_{y}\right) \exp \left(-y^{2} / 2 \sigma_{y}^{2}\right) \\
& \mathrm{dN}_{\mathrm{k}}\left(\mathrm{y}^{\prime}\right)=\left(\mathrm{U}_{1} \gamma^{2} \mathrm{H}_{2} \mathrm{dy^{ \prime }}\right)(\sigma s / \rho \sigma) \exp \left(-\mathrm{y}^{\prime} / 2 \sigma^{2}\right) \\
& d^{2} N_{k y}(0,0)=\left(U_{3} \gamma^{2} H_{2} d y d y^{\prime}\right)\left(s / p_{y}\right) \\
& \mathrm{dN}_{\mathrm{k}}(\mathrm{x})=\left(\mathrm{U}_{2} \mathrm{fG} \mathrm{G}_{1} \gamma \mathrm{dx}\right) \sigma_{\mathrm{x}}^{-1} \exp \left(-\mathrm{x}^{2} / 2 \sigma_{\mathrm{x}}^{2}\right)
\end{aligned}
$$

Continuing the table for $y=\lambda_{c} / \lambda=1, \theta= \pm 1$ mrad, unit current:
$\mathrm{d}^{2} \mathrm{~N}_{\mathrm{ky}}(0,0) \sim \frac{{\gamma^{2} \mathrm{~s}}^{2}}{\rho \sigma_{\mathrm{y}}}$
A
B
C
$\mathrm{d}^{2} \mathrm{~N}_{\mathrm{kx}}(0,0) \sim \gamma / \sigma \mathrm{x}$
1.9
38
7

These functions omit common multipliers to show proportionality. The $x, x^{\prime}$ densities are not a function of $s($ or $\theta$ ) but the total flux is, of course, proportional to $s$. Sagittas are sma1l compared to $\sigma_{x}$ and can be neglected up to at least $\pm 3 \mathrm{mrad}$. The y densities are proportional to $\theta=s / \rho$ and will increase if $\theta$ is increased, but they will spread over more $x^{\prime}$. However, the linear approximation will begin to fail at a few mrad for the low-emittance low- $\beta$ example, and at some tens of mrad for the large enittance example. The three examples are shown as $y, y^{\prime}$ and $x, x^{\prime}$ contours in Fig. 3. The source is 
the effective optical source at $s=0$, and is shown transformed 5 into the image space where it can fall on a slit, or crystal.

VyV sources usually subtend as much $\theta$ as the entrance of the instru* ment will accept. Examples are a low field wigglex in a $\sim 2 \mathrm{GeV}$ ring insertion, and the arc and an insertion of a typical $700 \mathrm{MeV}$ ring. optics of $7.5 \mathrm{~cm}$ diameter at $4 \frac{1}{2} \mathrm{~m}$ and $1 \frac{1}{2} \mathrm{~m}$ respectively are assumed to collect \pm 8 mrad and \pm 25 mrad.

D

Low Eield wiggler

$\gamma$

$\lambda_{c}(\AA)$

$\rho(m)$

$s(m)$

$\sigma_{y}(\mathrm{~mm})$

$\sigma_{y}(\operatorname{mrad})$

$\sigma(\mathrm{mm})$

$\mathrm{s}^{2} / 2 \rho(\mathrm{mm})$

$\sigma$ (mrad)

"I
$2936(1.5 \mathrm{GeV})$

32

19

$\pm 0.153$

0.006

0.025

0.15

0.61

0.194

0.53
E

$700 \mathrm{MeV}$ arc

1370

32

2

$\pm 0.049$

0.1

0.045

0.4

0.62

0.416

1.13
F

$700 \mathrm{MeV}$ insertion

1370

32

2

$\pm 0.049$

0.011

0.124

0.29

0.62

$0.416 y=1$

$1.13 \quad y=0.1$

In these l.ow emittance rings $\sigma_{\mathrm{y}}$, is small so $\sigma_{\mathrm{Y}^{\prime}}=\sigma$. The central densities are, continuing;

$\begin{array}{ccccc}\mathrm{d}^{2} \mathrm{~N}_{\mathrm{ky}}(0,0) \sim \frac{\gamma^{2} \mathrm{H}_{2} \mathrm{~s}}{\rho \sigma_{\mathrm{y}}} & \mathrm{D} & \mathrm{E} & \mathrm{F} & \\ \mathrm{d}^{2} \mathrm{~N}_{\mathrm{kx}}(0,0) \sim \frac{\gamma f G_{1}}{\sigma_{\mathrm{x}}} & 6.9 & 0.69 & 6.2 & \mathrm{y}=1 \\ & 11.2 & 0.28 & 2.6 & \mathrm{y}=0.1 \\ & 12.7 & 2.0 & 2.7 & \mathrm{y}=1 \\ & & 2.2 & 3.0 & \mathrm{y}=0.1\end{array}$

The effective source $\sigma_{y}$ is approximately double the beam $\sigma_{y}$ in examples $D$ and $F$ due to the effect of source length combined with a low $\beta$. (Solution of Eq. 2 for $\left.\mathrm{Y}>\mathrm{I}_{0}\right)$ The small beam size partially compensates for this. Source length makes only a small increase in example E. The source contours are shown in Eig. 3 . If the source is imaged by focussing optics the ratio of size to angle is changed by the magnification but the product can be reduced only by triming flux with an aperture. Aberrations distort the shape and can make the apparent size or angle increase. An image of the radial 
source would reproduce the $U$ shaped figure. After a drift space the $x, x^{\prime}$ contours straighten out as shown in example D of Fig. 3 and it is in principle possible to use non-linear optics to produce a straight image, but not to reduce its phase space area. However, the apparent $x, x^{\prime}$ area of example $D$ (maximum angle times maximum size) is much greater than the source area.

The most important optical characteristic of a storage ring light source is small emittance. Very small emittance is undesirable in a colliding beam ring but, fortunately, can be achieved in the design of a single beam ring. The angular emission of the source is the quadrature sum of the radiation divergence and electron beam divergence. Consequently the beam size can be reduced until the beam divergence is nearly half that of the radiation without much increase of source divergence. Extreme reduction of beam size with very low- $\beta$ is only useful for short sources due to the $y$ broadening by source length. (Imperfections of the storage ring also contribute beam broadening and it is desirable to plan careful fabrication and correction of the ring.) A small radius of curvature is desirable, especially in the $\mathrm{x}, \mathrm{x}^{\prime}$ plane. The brightest sources for high resolution wrsk will always be in insertions because double-focussing $(x, y)$ can be done in an insertion and the momentum dispersion can be nearly zero. This consideration indicates as many insertions as the orbit design (and finances) can tolerate. 


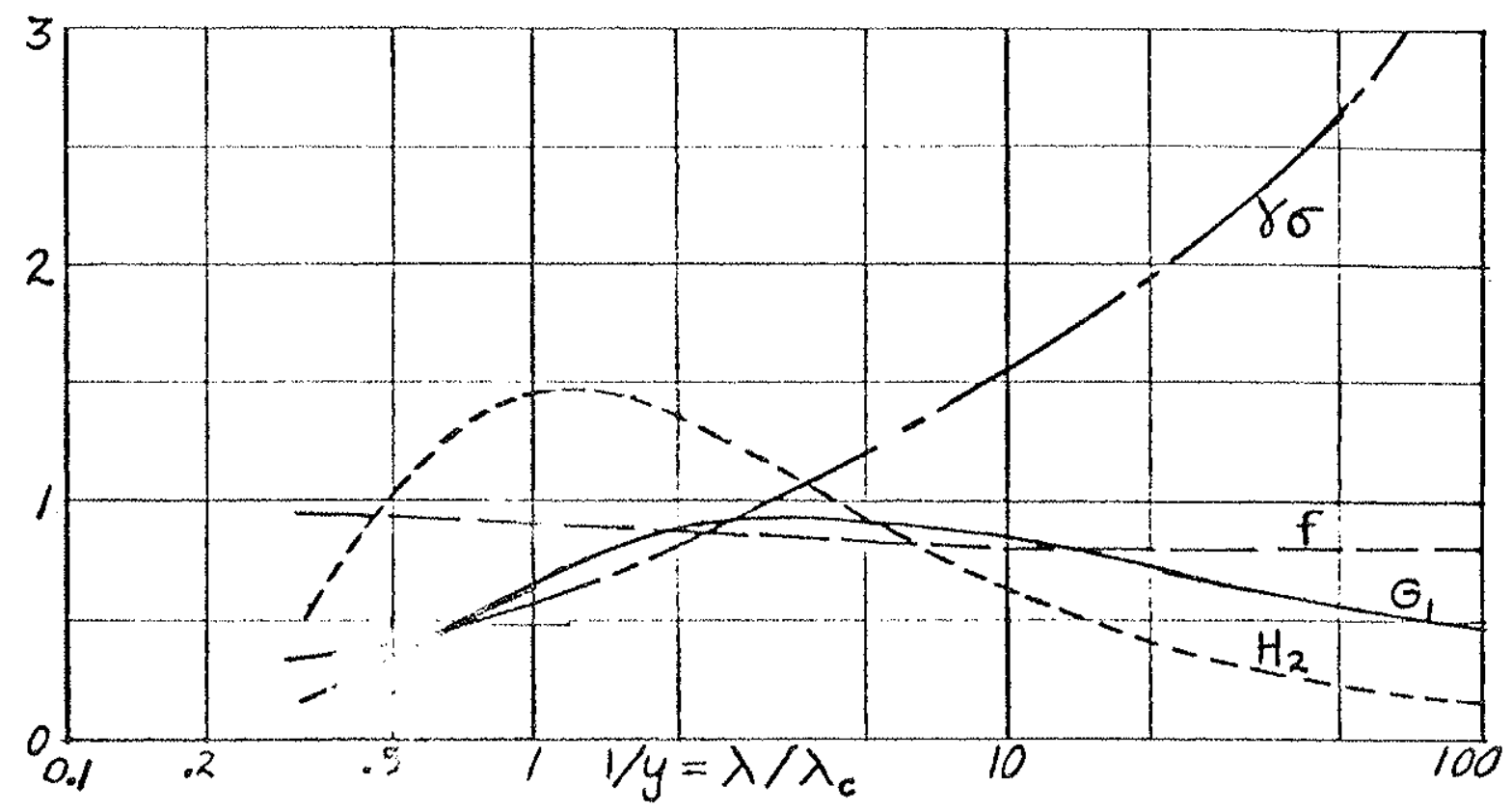

Fig. 1. Synchrotron radiation functions.
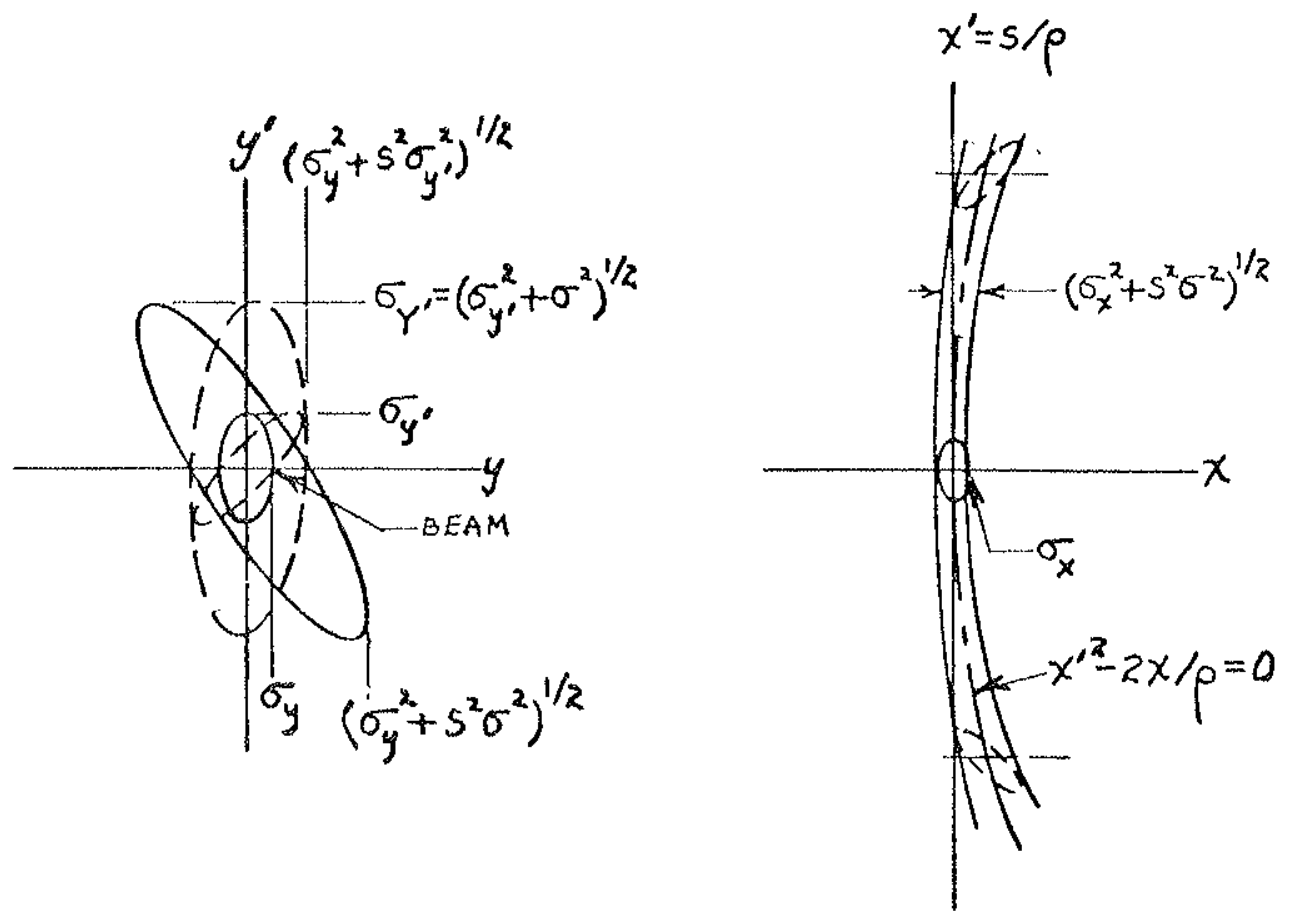

Fig. 2. Successive transformations in phase space. $s=0,---s=s$ 


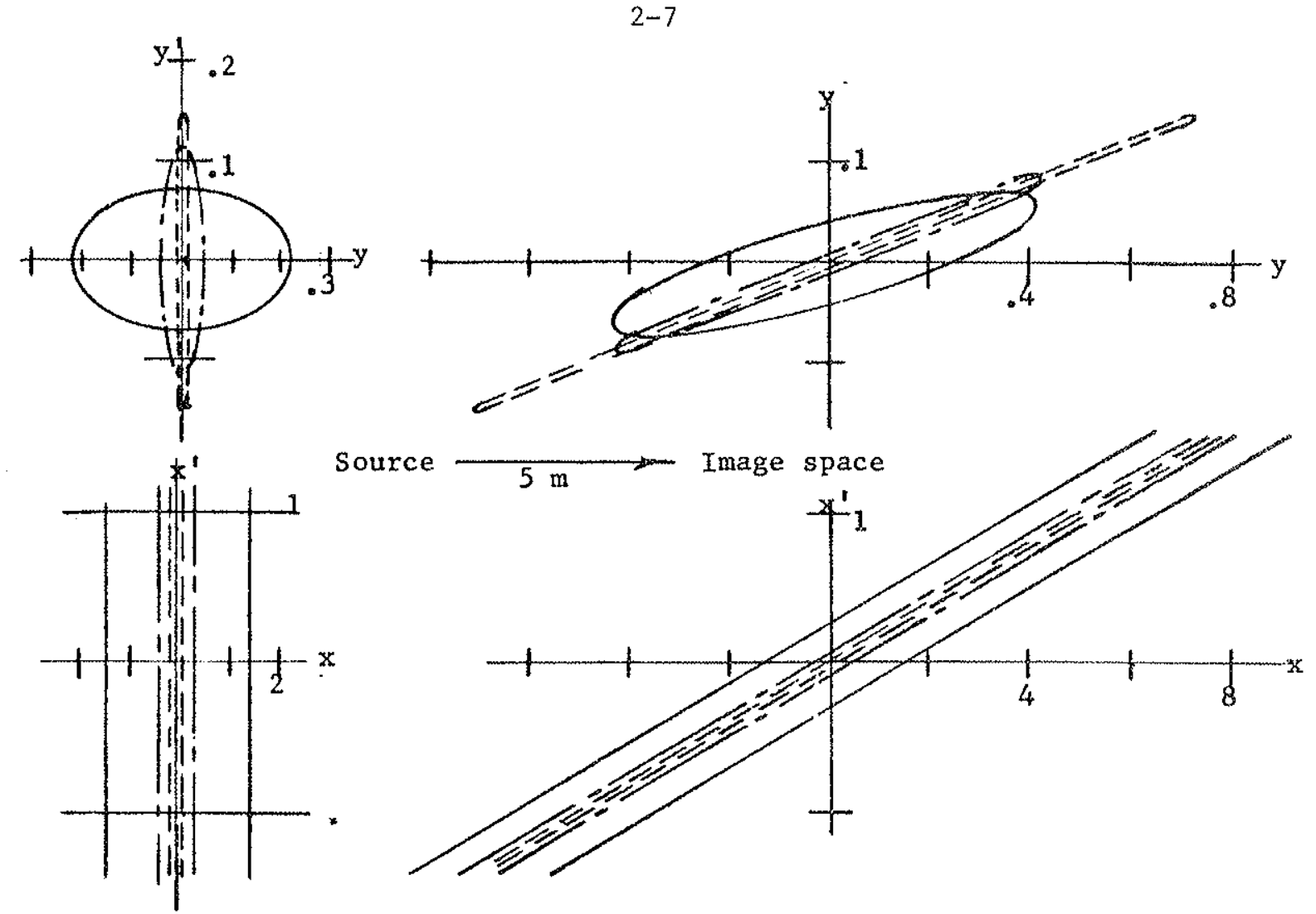

Fig. 3. $X$-ray soirces. $A--B,-B-m$.

Al1 scales in m, urad.
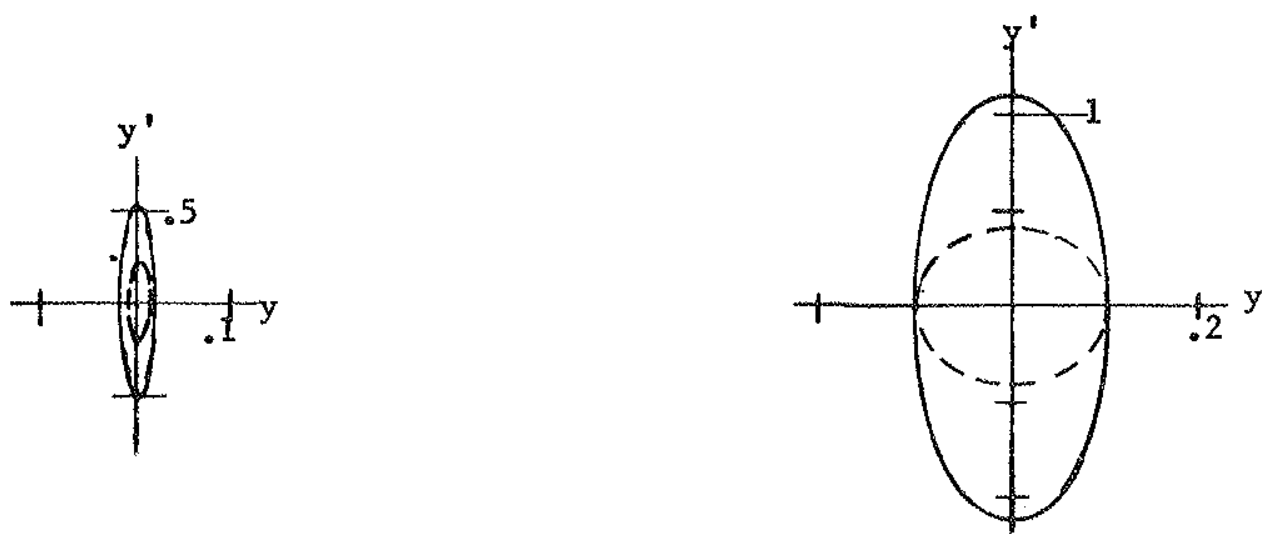

All scales in mm, mrad.
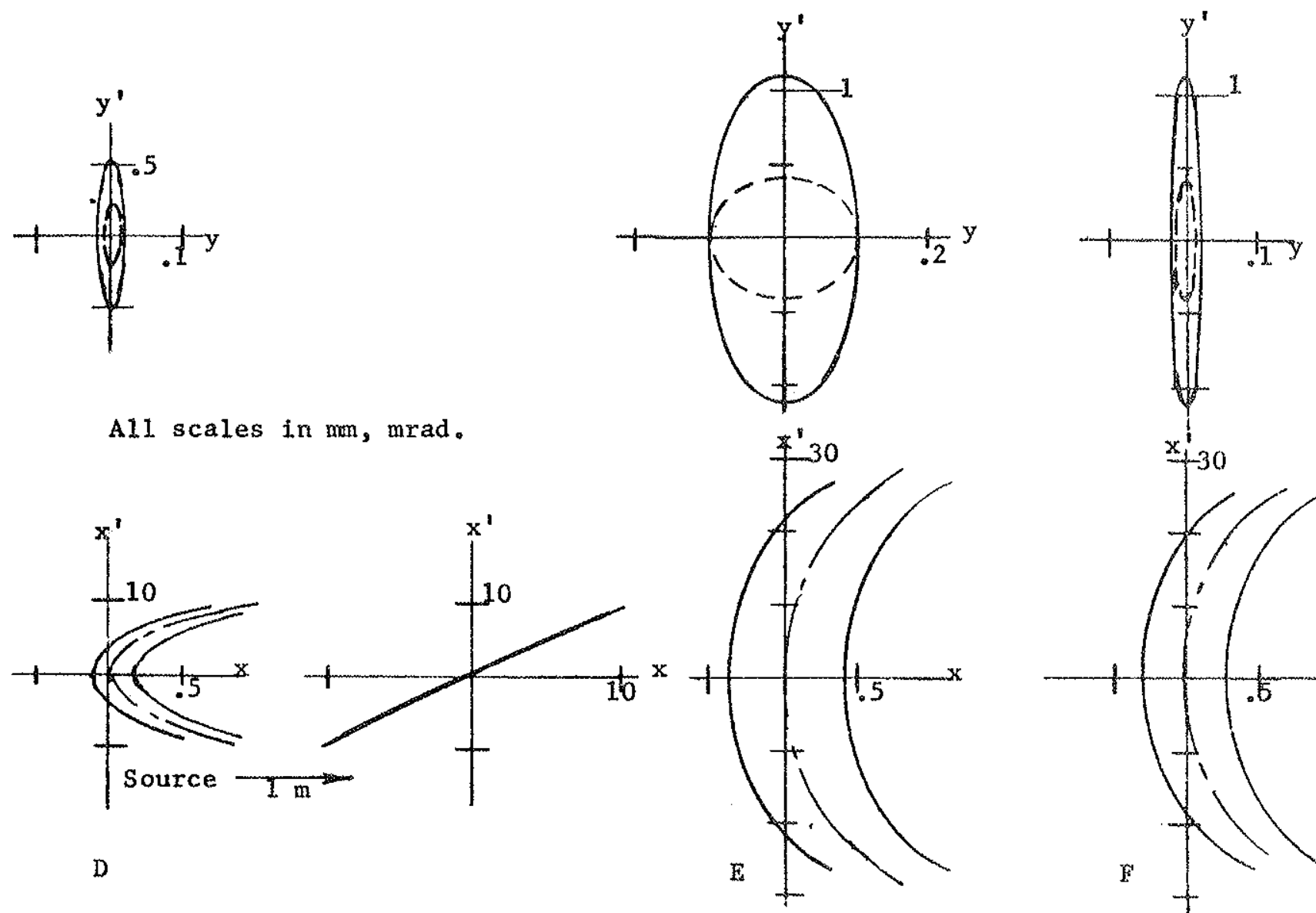

D $y=0.1$

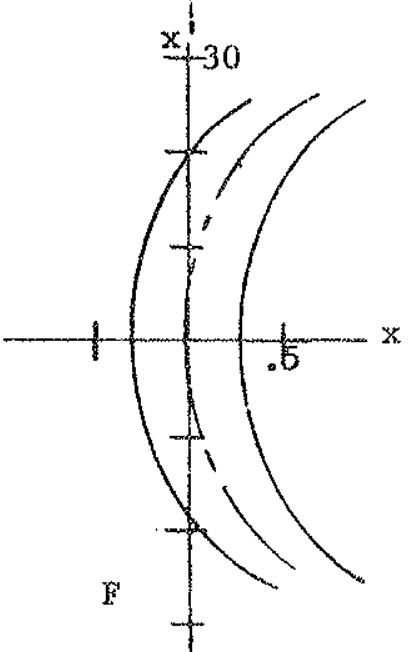

Fig. 4. VUV sources. $---y^{=1}$, 

Section 3.

A SHORT PERIOD HELICAL WIGGLER AS AN IMPROVED

SOURCE OP SYNCHROTRON RADIATION

Brian M. Kincaid

Bell Laboratories

Murray Hill, New Jersey 07974

\section{ABSTRACT}

A new kind of wiggler is proposed as an improved source of synchrotron radiation from high energy electron storage rings. The electrons are made to travel in a short period helix by a transverse helical magnetic field. The radiation spectrum produced is calculated and it is shown that the helical wiggler design can produce a total intensity (photons/sec/unit bandwidtr) improvement of several hundred and a brightness (photons/ sec/solid angle/bandwidth) improvement of $4 \times 10^{4}$ over the present state of the art in synchrotron radiation sources. 

A SHORT PERIOD HELICAL WIGGLER AS AN IMPROVED

SOURCE OF SYNCHROTRON RADIATION

Brian M. Kincaid

Bell Laboratories

Murray Hill, New Jersey 07974.

\section{INTRODUCTION}

Electrons travelling in curved orbits in storage rings emit synchrotron radiation due to the accelerations produced by the bending magnets. Almost all the radiofrequency power used to accelexate the electrons ends up as synchrotron radiation. Until recently this waste radiation has been merely an amoyance for accelerator designers, but it is now becoming an important research tool. The radiation emitted by an electron beam pasing through a bending magnet has two major advantages over conventional $X-r a y$ and UV sources: broad bandwidth and much higher apparent brightness (power or photons/sec produced per unit bandwidth per unit solid angle of detector). In $x$ way diffraction experiments or in high resolution spectroscopy, the highly collimated photon beam from a storage ring can result in many orders of magnitude increase in signal over conventional $X$-ray tube sources. At present, however, it is possible to produce more intensity (total photons/second per unit bandwiath) by collecting radiation from a conventional source over a large solid angle using a focusing arrangement than can be 
obtained from synchrotron radiation. sources. In some experiments, such as inelastic $x$-ray scattering or some kinds of biological $\mathrm{X}$-ray diffraction work, where source brightness is not very important, a conventional $X$-ray tube can, in pact, be great]y superior to present storage ring sources.

Periodic magnetic field devices, or "wigglers", have been proposed as a method of increasing the total output power of synchrotron sources. In the wiggler designs proposed to date, large magnetic field of about 30 Kgauss or more are applied in an alternating periodic array, usually with a period of several inches, imposed by magnet design limitations. ${ }^{1}$ In a wiggler of this kind the electrons feel more violent accelerations than in a normaj, storage ring bending magnet, and thus radiate more total power. Since the accelerations alternate in direction, the total angular deviation of the beam is smaller than in a normal bending magnet, so the radiated power is confined to a smaller solid angle, producing an increased intensity. Such a wigglex produces a normal synchrotron radiation spectrum with a critical energy, Ec, determined by the radius of curvature of the rlectron's orbit in each section of the wiggler and the slectron's energy. 2

This paper describes the properties of a special wiggler with a helical magnetic field design permitting 
much shorter magnet periods, and, as a rosult, the radiation produced has not unly a higher brightness and intensity than can be achieved using a conventional wigrler, but also a different power spectrum, with the radiation concentrated in a relatively narrow bandwidth. Such a wiggler installed at the SPIAR storasc ring could produce a brightness improvement of about $4 \times 10^{4}$ and a total intensity improvement (photons/sec per unit bandwidth) of several hundred over present SPEAR performance. This improvement would remove the advantage of even the most powerful conventional $x$-ray sources and would make possible experiments which are currently not being done because of source limitations.

Helical magnet design, the relative performance of helical wigglers, normal wigglers, and benuing magnets as synchrotron radiation sunces, and the possiblo eldects of a helical wigelur on the operation of the storage ring are discussed in the next sections. It should be noted that the terms brightness and intensity used in this paper refer to the radiation produced by a single electron. In an actual storage ring, however, the source brightness and intensity are determined by the angular divergence and the transverse dimensions of the radiating electron beam as well as the single electron properties discussed here. Some of the other effects of beam divergence on the radiation from the helical wigglers will also be aiscussed.

II. HELICAL MAGNETIC FIELDS AND SYNCHROTRON RADIATION FROM A HELICAL ELECTRON ORBIT

A transverse pexiodic helical magnetic field with constant magnitude is produced on the axis of a 
double helix wound bifilar magnet (Figure 1). Madey ${ }^{3}$

has used such a magnet in a study of stimulated emission of radiation by an electron beam in a periodic field. It can be shown ${ }^{4}$ that the transverse field on axis due to a single pair of current carrying wires wound in a bifilar helix is

$$
B_{1}=\frac{8 \pi I}{10 \lambda_{0}}\left[\frac{2 \pi a}{\lambda_{0}} K_{O}\left(\frac{2 \pi a}{\lambda_{O}}\right)+K_{1}\left(\frac{2 \pi a}{\lambda_{O}}\right)\right] .
$$

Here $B_{1}$ is in gauss, $I$ is the current in amps, $\lambda_{0}$ is the magnet period and a is the radius of the helix, both in $\mathrm{cm}$. $K_{O}$ and $K_{1}$ are modified Bessel functions. As $\frac{2 \pi a}{\lambda_{0}}$ increases for larger magnet bore diameters with fixed period, the on axis transverse field therefore decreases exponentialiy, making it necessary that the bore diameter and the period be roughly equal. Using superconducting technology, it should be possible to make a magnet producing 10 Kauss on axis with a period of about $1 \mathrm{~cm}$ and a bore diameter of about $1 \mathrm{~cm}$.

The orbit of a relativistic electron in a helical magnetic field is also a helix, with the same period, $\lambda_{0}$, and a radius determined by the centripetal acceleration produced by the magnetic field, given by

$$
\begin{aligned}
r_{H e \perp i x} & =\frac{\left(\frac{\lambda_{0}}{\lambda m_{\rho}}\right)^{2}}{\sqrt{1-\left(\frac{\lambda_{0}}{2 \pi \rho}\right)^{2}}} \\
& \approx \frac{\lambda_{0}^{2}}{4 \pi^{2}},
\end{aligned}
$$




$$
\rho=\frac{2 \beta m c^{2}}{e B}
$$

The pitch angle of the helix $\theta_{\text {pitch }}$ is given by

$$
\theta_{\text {pitch }} \approx \frac{2 \pi r}{\lambda_{0}}=\frac{\lambda_{0}}{2 \pi p} .
$$

For relativistic electrons, $\beta \approx 1$, and $\theta_{\text {pitch }}$ is given by

$$
\theta_{\text {pitch }}=\frac{\lambda_{0} e B}{2 \pi \gamma m^{2}} \equiv K / \gamma
$$

Synchrotron radiation is emitted along the direction of motion of the electrons with a small opening angle $\theta \approx 1 / \%$. Hence, for $K<1$ (weak field case), the pitch angle of the helical orbit is smaller than $1 / \gamma$ and the radiation is emitted into an angle of $1 / \gamma$. For $K>1$ (strong field case), the radiation is emitted into a cone of half angle $K / y$. See Figure 2 .

Since the total power emitted is proportional to $\mathrm{B}^{2}$, one can see that in the weak field case the power per unit solid angle increases with $B^{2}$ until one reaches the strong field limit, where the power and the solid angle both increase as $B^{2}$.

using a trick of Purceld ${ }^{2}$ we can view the electron's motion from an inertial frame moving in the $+\widehat{z}$ direction at the average speed of the electron, $v=\beta^{*} c$, where 


$$
\beta^{*}=\beta \sqrt{1-\left(\frac{\lambda_{0}}{2 \pi p}\right)^{2}}=\beta \sqrt{1-\left(\frac{K}{\gamma}\right)^{2}} .
$$

In this frame an electron in the weak fleld limit is traveling in a circular orbit with nonrelativistic velocity. It therefore emits a nomal dipole pattern of circularly polarized single frequency radiation. See Figure $3 a$. This radiation will also look monochromatic in the lab frame, but its frequency and intensity will vary with the viewing angle due to the doppler effect. See figure 30. This is derived in detail in the appendix. In the strong field case, however, the electron actually has a relativistic orbital velocity in the moving frame, and it therefore emits synchrotron radiation in this frame, with a different angular distribution and a rrequency spectrum containing harmonics. See figure 3c. Back in the lab frame, an observer sees a spectrum of harmonics, each one of which has a frequency and amplitude which varies with viewing angle, shown in Figure 3d. The intrinsic angular divergence of an electron beam in a storage ring smears out the spectrum into a normal synchrotron radiation spectrum ${ }^{2}$ having a critical. energy, sc, given by

$$
E_{c}=\frac{3}{2} \frac{h c}{\rho} y^{3}
$$

with $p$ being the cyclotron radius of the electron's orbit. In order to compare helical wiggler synchrotron radiation sources with normal wigglers and bending magnets, 
the radiation distribution in frequency and angle for an electron moving in a helical orbit must be derived. The energy radiated per unit bandwidth by one electron into a solid angle do (brightness per electron), $\frac{d I(\omega)}{d}$, is given by

$$
\frac{d I(\omega)}{d \hat{n}}=\frac{e^{2} \omega^{2}}{4 \pi^{2} c}\left|\int_{-\infty}^{+\infty} \hat{n} \times(\hat{n} \times \hat{\beta}) e^{i \omega\left(t-\frac{\hat{m} \cdot \vec{r}(t)}{c}\right)} d t\right|^{2} .
$$

Here $\vec{r}(t)$. is the vector describing the path of the electron, $\vec{\beta}(t)$ is $\frac{\dot{\vec{r}}(t)}{c}$, and $\hat{n}$ is a unit vector pointing from the origin to the observer. See figure 4. If the observer is at an angle $\theta$ in the $y-z$ plane, and if the radius of the helical orbit is denoted by a, then we have

$$
\begin{aligned}
\hat{n} & =\hat{z} \cos \theta+\hat{y} \sin \theta, \\
\vec{r}(t) & =\beta^{*} \operatorname{ct} \hat{z}+a \sin \omega_{0} t \hat{y}+a \cos \omega_{0} t \hat{x}, \text { and } \\
\vec{\beta}(t) & =\beta^{*} \hat{z}+\frac{a \omega_{0}}{c} \cos \omega_{0} t \hat{y}-\frac{a(x)}{c} \sin \omega_{0} t \hat{x} .
\end{aligned}
$$

As previously, $\beta^{*}=\beta \sqrt{1-\left(\frac{K}{\gamma}\right)^{2}}$ and

$$
a=\frac{\left(\frac{k}{y}\right)^{2} \rho}{\sqrt{1-\left(\frac{k}{y}\right)^{2}}}
$$


Here $\omega_{0}$ is $\frac{2 \pi \beta^{*} c}{\lambda_{0}}$, the circular frequency of the electron's nelical orbit.

It is interesting to note that one need not make any approximations to evaluate this integral. However, no real validity is lost if several simplifying assumptions are made. First, $\gamma$ is assumed to be large, so that $K / \gamma$ is small for all reasonable choices of $K$. In addition, since it is known that the radiation pattern is peaked shaxply for angles near $\theta \approx 1 / \gamma$, we may approximate $\sin \theta$ and $\cos \theta$ by their small angle forms. The number of periods or wiggles in the magnet is assumed to be reasonably large, $(N \approx 100)$, enabling the final expressions to be further simplified. Using these approximations the expression for $\frac{d I(\omega)}{h}$ becomes (see $\Lambda$ ppendix):

$$
\begin{aligned}
& \frac{d I(\omega)}{\partial \sigma}=\frac{e^{2} \omega^{2} K^{2}}{\pi^{2} c \omega_{0}^{2} \gamma^{2}} \sum_{n=1}^{\infty}\left[J_{n}^{2}(x)+\left(\frac{\gamma \theta}{K}-\frac{n}{x}\right)^{2} J_{n}^{2}(x)\right] \\
& \times \frac{\sin ^{2} N \pi\left(\frac{\omega}{\omega_{1}}-n\right)}{\left(\frac{\omega}{\omega_{1}}-n\right)^{2}} .
\end{aligned}
$$

Here $x=\frac{k \omega \theta}{\gamma \omega_{0}}$

$$
\omega_{1}=\frac{\omega_{0}}{1-\beta^{*} \cos \theta} \simeq \frac{2 \gamma^{2} \omega_{0}}{1+K^{2}+\gamma^{2} \theta^{2}}
$$


and $J_{n}$ and $J_{n}^{\prime}$ are the nth order Bessel function and its derivative. Thus the spectrum consists of a series of harmonics of the fundamental frequency $\omega_{1}$, with the intensity of the nth harmonic given by the nth term in the series. For $\theta=0$ (on axis) all the higher harmonics are seen to vanish, and we are left with a spectrum consisting of a single narrow peak at

$$
\omega=\omega_{1}=\frac{2 \gamma^{2} \omega_{0}}{1+K^{2}} .
$$

In terms of wavelength, the spectrum has a peak at

$$
\lambda=\frac{\lambda_{0}}{2 \gamma^{2}}\left(1+K^{2}\right) .
$$

For $K=1, \gamma=10^{4}$, and $\lambda_{O}=1 \mathrm{~cm}, \lambda$ then is $1 \stackrel{\circ}{\mathrm{A}}$.

The fractional Inewidth (FWHM) is about $1 / \mathrm{N}$, or $1 \%$ for a 100 period wiggler. This corresponds to a $120 \mathrm{eV}$ bandwidth for the $1 \stackrel{\circ}{\mathrm{A}}$ case above. Of course, the only way to actually realize such a small bandwidth is to make sure that the other factors contributing to the bandwidth are all smaller than $1 / \mathrm{N}$, including energy purity of the electron beam (typically .1\% in a storage ring), the magnetic field homogeneity in the wiggler (this changes the value of $K$ seen by different parts of the electron beam), the angular size of the detector (it must be smaller than $\theta \approx \frac{1}{\sqrt{\mathbb{N}} \gamma}$, and finally, the angular 
divergence of the ejectron bean, which again must be smallex than $\theta \approx \frac{1}{\sqrt{N} y}$ for the $\gamma^{2} \theta^{2}$ term in $\omega_{1}$ to be less than 1/N. This last condition is probably the most stringent requirement on the design of a storage ring ior use with a helical wiggler. It does seem possible, however, to achieve the small angular divergence using special focusing inserts. 6 the effect of electron beam divergence on the spectral purity of the radiation will be investigated later. The effect of magnetic field homogeneity is minimized by the requirement that the electron beam be small compared to the diameter of the beam pipe through the center of the magnet. This is necessary to achieve a long lifetime for a stored beam in a storage ring. 7 With these caveats we may now examine the on axis brightness function, equation (10), in more detail. For $\omega=\omega_{1},(10)$ becomes

$$
\left.\frac{d I(\omega)}{\partial n}\right|_{\substack{\omega=\omega_{1} \\ \theta=0}}=\frac{2 N^{2} e \gamma^{2} K^{2}}{c\left(1+K^{2}\right)^{2}} .
$$

$K=1$ maximizes this expression, yielding

$$
\left.\frac{d I(\omega)}{d \Omega}\right|_{\substack{\omega=1 \omega_{1} \\ \theta=0 \\ K=1}}=\frac{N^{2} e^{2} y^{2}}{2 c} .
$$


Dividing by $N \lambda_{0}$ to get brightness/cm, and converting to more practical units, we have

$$
\begin{aligned}
\frac{d I(\omega)}{d \Omega} /_{N \lambda_{0}}= & 2.94 \times 10^{-8} \cdot \mathrm{hv} \mathrm{ev}^{\cdot i_{\mathrm{ma}} \cdot \mathrm{N}} \\
& \text { watts/ev of bandwidth } \\
& / \mathrm{ma} /(\mathrm{milli} \text { radian })^{2} / \mathrm{cm}, \\
\text { or, } \quad & \\
& 1.84 \times 10^{9} \cdot \mathrm{hv} \mathrm{ev} \cdot \mathrm{i}_{\mathrm{ma}} \cdot \mathrm{N} \\
& \text { photons } / \mathrm{sec} / 1 \% \text { bandwidth } \\
& / \mathrm{ma} /(\mathrm{mrad})^{2} / \mathrm{cm},
\end{aligned}
$$

where $h \nu_{e v}$ is the photon energy in eV corresponding to $\omega_{1}$ and $i_{m a}$ is the electron beam current in milliamps. III. COMPARISON WITH BENDING MAGNET AND NORMAL WIGGLER

The brightness function for a normal bending magnet source has been derived ${ }^{5}$ and is given by:

$$
\left.\frac{d I(\omega)}{d n}\right|_{\theta=0}=\frac{3 e^{2}}{4 \pi^{2} c} r^{2} y^{2} K_{2 / 3}^{2}(r / 2)
$$

where $r=h v / E_{c}$ and $E_{c}$ is the critical energy of the spectrum given by (7).

$$
\text { In the case of a normal strong field wiggler, }
$$
as described previously, one has the freedom to adjust the magnetic field and hence $E_{c}$ in each section of the wiggler so as to maximize $r^{2} k_{2 / 3}^{2}(r / 2)$. This occurs at $r=1$ with a maximum value of approximately 1.5. Under these conditions, the brightness is: 


$$
\frac{d I(\omega)}{d o}=2 N \cdot \frac{3 e^{2} y^{2}}{4 m^{2} c} \cdot\left[x^{2} K_{2 / 3}^{2}(x / 2)\right]_{\max } \text {, }
$$

with $N$ being the number of periods. The numerical values of these brightness formulas for the three cases are compared in Figure 5. The units of brightness used are watts/ev of bandwidth/ma of electron beam/(milliradian $)^{2}$ of detector solid angle. To get photons/second/1\% bandwiath instead of watts/ev, multiply by $6.24 \times 10^{16}$. The brightness functions axe plotted for electron beam energies of .5 , 1.0, 2.0 and $4.0 \mathrm{Gev}$. For the helical device, brightness per $\mathrm{cm}$ of wiggler is plotted for a wiggler with 200 periods and $K=1$, conditions one might actually encounter in a practical application. The peak helical wiggler brightness is independent of $\gamma$, so there is only one curve vs four curves for each of the other two methods. The vertical bars on the brightness curves represent technological or design limits. For the helical wiggler, the output photon energy depends on $\lambda_{0}$, and therefore if $\lambda_{0} \geq 1 \mathrm{~cm}$, there is a maximum photon energy for each electron energy. Similarly, if one places a limit of 50 Kgauss on normal wiggler magnets, there is a maximum critical energy for each value of $\gamma$. The brightness curve for the normal wiggler is plotted as brightness/wiggle (1. period $=2$ wiggles), and, as expected, it touches the curve for the bending magnet at its maximum value. A field of 10 Kgauss is assumed for the bending 
magnet case. In a practical helical wiggler application, one would choose $\lambda_{0}$ as small as possible to maximize $N$ for a given length of wiggler, $N \lambda_{0}$, then adjust the magnetic field to get $K=1$, and tune the output photon energy by varyine $\gamma$. For a fixed electron energy it is, of course, possible to tune the photon energy by varying $K$ silghtly, at the expense of reduced brightness and spectral purity as $K$ increases beyond $K=1$. Referring again to Figure 5 , one can see that a helical wiggler with $\lambda_{0}=1 \mathrm{~cm}$ and $N=200$ has a potential brightness advantage of about $10^{4}$ over a bending magnet, assuming that the desired photon energy is within the limits of the helical wiggler. The serious practical limit on the brightness of the helical. wiggler output is the angular divergence of the electron beam due to the intrinsic properties of storage rings. 7 Since the output frequency of the radiation is a function of $\theta$ according to (11), a distribution of electron beam angles will spread the radiation at a given frequency over a range of angles and hence reduce the brightness. In addition, since the amplitudes of harmonics depend on $\theta$, there will be some degradation of spectral purity.

If one assumes a normalized gaussian distribution of angles, 


$$
P(\theta)=\frac{1}{2 \pi \sigma^{2}} e^{-\frac{\theta^{2}}{2 \sigma^{2}}},
$$

and considers cases where

$$
\sigma^{2}>\frac{1}{N \gamma^{2}}
$$

then the electron beam divergence will be the dominant factor in the brightness formula. One is then entitled to replace

$$
\frac{\sin ^{2} N \pi\left(\frac{\omega}{\omega_{1}}-n\right)}{\left(\frac{\omega}{\omega_{1}}-n\right)^{2}} \text { by }
$$

and to convolve (10) with the gaussian distribution (19), ylelding, for $\theta=0$ (on axis), (see appendix for details)

$$
\begin{aligned}
\left.\frac{d I(\omega)}{d n} * P(\theta)\right|_{\theta=0} & =\frac{2 N e^{2} K^{2} \gamma^{2} r}{\sigma_{\alpha}^{2} c} \sum_{n=1}^{\infty}\left(J_{n}^{\prime 2}\left(x_{n}\right)+\right. \\
& \left.+\left(\frac{\alpha_{n}}{K}-\frac{n}{x_{n}}\right)^{2} J_{n}^{2}\left(x_{n}\right)\right) e^{-\frac{\alpha_{n}^{2}}{2 \sigma_{\alpha}^{2}} \theta\left(\alpha_{n}^{2}\right),}
\end{aligned}
$$


where $\sigma_{\alpha}=\gamma \sigma, \alpha=\gamma \theta, \alpha_{n}^{2}=\frac{n}{\gamma}-1-k^{2}, x_{n}=2 k r \alpha_{n}$, and $r=\omega / 2 \gamma^{2} \omega_{0} \cdot \theta\left(\alpha_{n}^{2}\right)$ is the unit step function.

This formula is valid for $\sigma_{\alpha}^{2} \gg \frac{1}{N^{2}}$. For

$\sigma_{\alpha}^{2} \ll \frac{1}{N},(10)$ holds. From (23) one can see that the brightness at the peak of the spectrum at

$$
\omega=\frac{2 \gamma^{2} \omega_{0}}{1+K^{2}}
$$

has been reduced by a fractor of $2 \mathrm{~N} \sigma_{\alpha}^{2}$, as expected. Figure 6 shows expression (23) plotted for $\theta=0, K=1$ and two values of $\sigma_{\alpha}$, and shows not only the reduced peak height and increased width of the spectrum, but also the larger percentage of harmonics for large $\sigma_{a}$. The charp edge in the spectrum is a consequence of the approximation that $\mathbb{N}$ is large and that

$$
\frac{\sin ^{2} N \pi x}{x^{2}}
$$

may be treated as $N \pi^{2} \delta(x)$. If the expressions were evaluated without this approximation, the sharp edges would be rounded, with a step width of $\approx \frac{1}{N}$.

Expression (10) may be integrated over all positive frequencies, yielding a formula for the angular distribution of radiated power: 
$\frac{d W}{d \theta}=\frac{8 \mathrm{Ne}^{2} \omega_{0} \gamma^{4}}{c\left(1+K^{2}+y^{2} \theta^{2}\right)^{3}} \sum_{n=1}^{\infty} n^{2}\left(J_{n}^{2}\left(x_{n}\right)+\left(\frac{\gamma \theta}{K}-\frac{n}{x_{n}}\right)^{2} J_{n}^{2}\left(x_{n}\right)\right)$,

where $x_{n}=\frac{2 K n y \theta}{1+K^{2}+\gamma^{2} \theta^{2}} \quad$ This is plotted as a function of $\alpha=\gamma \theta$ for several values of $K$ in Figure 7 . The decrease in intensity at $\alpha=0$ with large $K$ due to increasing helix pitch angle is clearly seen.

Expression (10) may also be integrated over all angles to produce an expression for the frequency spectrum:

$$
\begin{aligned}
I(\omega) & =\int \frac{d I(\omega)}{d \Omega} d \Omega \\
& =\frac{4 \pi N^{2} K^{2} y}{c} \sum_{n=1}^{\infty}\left(J_{n}^{\prime 2}\left(x_{n}\right)+\left(\frac{\alpha_{n}}{K}-\frac{n}{x_{n}}\right)^{2} J_{n}^{2}\left(x_{n}\right)\right) \theta\left(\alpha_{n}^{2}\right),
\end{aligned}
$$

with $\alpha_{n}^{2}=\frac{n}{r}-1-K^{2}, x_{n}=2 K r \alpha_{n}$, and $r=\frac{\omega}{2 \gamma^{2} \omega_{0}}$, as before. Figure 8 shows a plot of the peak values of the spectra for a helical wiggler, a normal wiggler, compared with the spectrum for a bending magnet ${ }^{8}$ under the same condition as in the brightness comparison. Ihe units are power/ev/ma/cm of beam viewed by the detector. Interestingly enough, the curves for the helical wiggler and normal wiggler coincide. The same design limits of $\lambda_{O} \geq 1 \mathrm{~cm}$ and $\mathrm{B}$ wiggler $\leq 50 \mathrm{Kgauss}$ are indicated. It should be realized, trough, that a 
focusing arrangement is required in order to collect radiation over more than just a few $\mathrm{cm}$ of arc using a normal wiggler or a bending magnet, where the elcctron orbit suffers a large deflection in the magnetic field. In the helical wiggler, deflections are small, and a single non-focusing detector still can collect radiation produced over the entire length of the wiggler. This gives a practical advantage of a factor of $N$ or about 100 for the helical wiggler.

The actual shape of the power spectrum for the helical wiggler is shown in figure 9 for several values of $K$ : The shift of the peak with increasing $K$ predicted by (26) is clearly visible.

As a practical comparison, Figure 10 chows the actual power spectra for the three devices under some typical conditions. Here the comparison assumes that the detector accepts all vertical angles and accepts one milliradian of horizontal angle, a reasonable chojce, since the radiation from the bending magnet and normal wiggler will have a small vertical extent. A field of lo Kgauss is assumed for the bending magnet, 6 poles of strength 50 Kgauss for the normal wiggler, and $200 \mathrm{I} \mathrm{cm}$ periods for the helica]. wiggler. The $y$-axis is a compressed log scale covering fourteen orders of magnitude. Interestingly enough, on 
this scale, the quasi-monochromatic character of the heljcal wiggler spectrum has been reduced to some small bumps riding on what looks like an ordinary synchrotron radiation spectrum.

The helical magnetic field produced by a double helix solenoid has focusing properties that as yet have not been worked out. Madey ${ }^{9}$ has observed the effect of this focusing on the $25 \mathrm{MeV}$ electron beam in his experiment. The focusing effect is apparently explained by the net average force produced on the electron due to its motion through the rapidly varying field gradient off axis in the helical magnet. 10 This results in a radial restoring force, $F(x)$, roughly proportional. to the distance from the orbit center to the axis of the magnet. Such a force will mix transverse betatron oscillations in a storage ring by some amount, thus altering the beam size and dynamjes in the ring.

Another side effect of all wigglers is increased radiation damping. This can affect the damping for transverse betatron oscillation and hence may affect the stability of the stored beam. ${ }^{7}$ one should be fairly safe, however, if the total power radiated by the beam over the entire storage ring is much greater than the power radiated in the wiggler section and if the wiggler is properly placed in the ring magnet lattice. 
SUMMARY AND CONCLUSIONS

The radiation from an electron beam in a short period helical wiggler has been shown to have several important qualitative differences from that produced in a more conventional way. It is quasi-monochromatic, with a fractional linewidth determined by electron beam divergence and by the number of periods in the helix. On axis the spectral purity is very good, an advantage over conventional methods in some experiments. The frequency of radiation is tunable over a large range by varying the electron beam energy and over a smallex range for fixed energy by varying the magnetic field. The radiation pattern produced is sharply peaked in the forward direction with an overall angular size of about $1 / \%$. In addition, the radiation at the peak of the power spectrum is emitted into an even smaller angle of $\theta \approx \frac{1}{\sqrt{\mathrm{N}} \gamma}$, thus producing a brightness improvement of at least $10^{4}$ over a conventional bending magnet synchrotron source. On axis the radiation is circularly polarized. The short period helical field produces less angular deviation of the electron beam than other methods, thus reducing or eliminating the necessity for focusing to achieve a high power spectral density, and yielding a power per unit bandwidth improvement of about 100 over conventional methods.

In conclusion, it should be pointed out that the limits imposed on the helical wiggler design, i.e. magnet technology and present electron storage ring design, may at 
some point in the future be overcome, either through advances in superconducting magnet technology or through improvements in permanent magnets, and it therefore may be possible to use a short period wiggler to generate large amounts of UV or $\mathrm{X}$-ray power using relatively low energy storage rings of on the order of 1 to $2 \mathrm{GeV}$, thus making synchrotron radiation a much more generaliy available resource.

Helpful conversations with $P$. Eisenberger on the concept of source brightress and with J. Madey about his work are gratefully acknowledged. 
I. Derivation of Equation (10) Starting from (8),

$$
\frac{d I(\omega)}{d \Omega}=\frac{e^{2} \omega^{2}}{4 \pi^{2} c}\left|\int_{-\infty}^{+\infty} \hat{n} \times(\hat{n} \times \vec{\beta}) e^{i \omega t\left(-\frac{\hat{n} \cdot \vec{r}(t)}{c}\right)} d t\right|^{2},
$$

and the definitions,

$$
\begin{aligned}
\hat{n} & =\hat{z} \cos \theta+\hat{y} \sin \theta, \\
\vec{r}(t) & =\beta^{*} \operatorname{ct} \hat{z}+a \sin \omega_{0} t \hat{y}+a \cos \omega_{0} t \hat{x}, \\
\vec{\beta}(t) & =\beta^{*} \hat{z}+\frac{a \omega_{0}}{c} \cos \omega_{0} t \hat{y}-\frac{a \omega_{0}}{c} \sin \omega_{0} t \hat{x}, \\
\beta^{*} & =\beta \sqrt{1-\left(\frac{\lambda_{0}}{2 \pi \rho}\right)^{2}}, \\
a & \left.=\frac{\frac{\lambda_{0}^{2}}{(2 \pi p)^{2}} p}{\sqrt{1-\frac{\lambda_{0}^{c}}{(2 \pi \rho)^{2}}}} \text { (radius of nelical orbit }\right) \\
\omega_{0} & =\frac{2 \pi c}{\lambda_{0}}, \text { and } \\
\rho & =\frac{B y m c^{2}}{e B}, \quad \text { (cyclotron radius) }
\end{aligned}
$$


and defining the magnetic field parameter, $K$, by

$$
K \equiv \frac{e \lambda_{0} B}{2 \pi m c^{2}} \approx 1 \text {, }
$$

we may write

$$
\begin{aligned}
& \hat{n} \times(\hat{n} \times \hat{\beta})= \\
& \hat{x}\left(\frac{K}{\gamma} \sin ^{2} \theta \sin \omega_{0} t+\frac{K}{\gamma} \sin \omega_{0} t \cos ^{2} \theta\right) \\
& +\hat{y}\left(\beta^{*} \sin \theta \cos \theta-\frac{K}{\gamma} \cos ^{2} \theta \cos \omega_{0} t\right) \\
& +\hat{z}\left(-\beta^{*} \sin ^{2} \theta+\frac{K}{\gamma} \cos \theta \sin \theta \cos \omega_{0} t\right) .
\end{aligned}
$$

Also,

$$
\frac{\hat{n} \cdot \vec{r}(t)}{c}=\beta^{*} t \cos \theta+\frac{a}{c} \sin \omega_{0} t
$$

as long as $\frac{-N \pi}{\omega_{0}} \leq t \leq \frac{N \pi}{\omega_{0}}$, for a helical orbit with $N$ periods. For $|t|>\frac{N \pi}{\omega_{0}}, a=0$ (no magnetic field), and

$$
\begin{aligned}
\hat{n} \times(\hat{n} \times \vec{\beta}) & =\hat{y}\left(\beta^{*} \sin \theta \cos \theta\right) \\
& +\hat{z}\left(-\beta^{*} \sin ^{2} \theta\right)
\end{aligned}
$$

and

$$
\frac{\widehat{n} \cdot \vec{r}(t)}{c}=\beta^{*} t \cos \theta
$$


We may break up (AI) into three pieces, yielding

$\int_{-\infty}^{+\infty}(\quad)=\int_{-\frac{N \pi}{\omega_{0}}}^{+\frac{N \pi}{\omega_{0}}}(\quad)+\int_{-\infty}^{+\infty}(a=0)-\int_{-\frac{N \pi}{\omega_{0}}}^{+\frac{N \pi}{\omega_{0}}}(a=0)$.

The second term is

$\int_{-\infty}^{+\infty}\left(\hat{z}\left(-\beta^{*} \sin ^{2} \theta\right)+\hat{y}^{*} \sin \theta \cos \theta\right) e^{i \omega t\left(1-\beta^{*} \cos \theta\right)} d t$,

and the third term is

$$
-\int_{-\frac{N \pi}{\omega_{0}}}^{\frac{N \pi}{\omega_{0}}}(\text { same }) .
$$

The second term then becomes

$$
\left(\hat{z}\left(-\beta^{*} \sin ^{2} \theta\right)+\hat{y}\left(\beta^{*} \sin \theta \cos \theta\right)\right) \cdot \frac{2 \pi \delta(\omega)}{1-\beta^{*} \cos \theta},
$$

and its contribution to (A.1) will vanish due to the $\omega^{2}$ term in front of $(A I)$. The third term is

$\left[\hat{z}\left(-\beta^{*} \sin ^{2} \theta\right)+\hat{y}\left(\beta^{*} \sin \theta \cos \theta\right)\right]\left(\frac{2 \sin N \pi \frac{\omega}{\omega_{0}}\left(1-\beta^{*} \cos \theta\right)}{\omega\left(1-\beta^{*} \cos \theta\right)}\right)$. 
This function has a maximum value of

$$
\left(\hat{z}\left(-\beta^{*} \sin ^{2} \theta\right)+\hat{y}\left(\beta^{*} \sin \theta \cos \theta\right)\right) \cdot \frac{2 \pi N}{\omega_{0}}
$$

at $\omega=0$. In addition, since $\theta$ is small $\left(\approx \frac{1}{\gamma}\right)$, the entire term will have only a small contribution to (AI).

Now, neglecting terms second order or higher in $1 / \gamma$ in the expression for $\hat{n} \times(\hat{n} \times \vec{\beta})$, we get

$$
\begin{gathered}
\frac{d I(\omega)}{d \hat{h}}=\frac{e^{2} \omega^{2}}{4 \pi^{2} c} \mid \int_{-\frac{N \pi}{\omega_{0}}}^{+\frac{N \pi}{\omega_{0}}}\left\{\dot{\hat{x}}\left(\frac{K}{\gamma} \sin \omega_{0} t\right)-\hat{y}\left(\beta^{*} \theta-\frac{K}{\gamma} \cos \omega_{0} t\right)\right\} \times \\
\\
\quad e^{i \omega t\left(1-\beta^{*} \cos \theta\right)}-\left.\frac{i \omega \mathrm{a}}{c} \sin 0 \sin \omega_{0}^{t} d t\right|^{2} .
\end{gathered}
$$

Writing $\frac{\omega a}{c}$ as $\frac{K \omega}{\gamma \omega_{0}}$, we get, squaring each vector component separately,

$\frac{d I(\omega)}{d \Omega}=\frac{e^{2} \omega^{2}}{4 \pi^{2} c}\left|\int_{-\frac{N \pi}{\omega_{0}}}^{\frac{N \pi}{\omega_{0}}} \frac{K}{\gamma} \sin \omega_{0} t e^{i \omega t\left(1-\beta^{*} \cos \theta\right)-\frac{i K \omega}{\gamma \omega_{0}} \sin \theta \sin \omega_{0} t} d t\right|^{2}$

$+\frac{e^{2} \omega^{2}}{4 \pi^{2}}\left|\int_{-\frac{N \pi}{\omega_{0}}}^{+\frac{N \pi}{\omega_{0}}}\left(\beta^{*} \theta-\frac{K}{\gamma} \cos \omega_{0} t\right) e^{i \omega t\left(1-\beta^{*} \cos \theta\right)-\frac{i K \omega}{\gamma \omega_{0}} \sin \theta \sin \omega_{0} t} d t\right|^{2}$ 
Changing variables to $t \leftarrow \omega_{0} t$, we get

$A=\frac{e^{2} \omega^{2}}{4 \pi^{2} c}\left|\int_{-N \pi}^{+N \pi} \frac{k}{2 \omega_{0}} \sin \tau e^{\frac{i \omega t}{\omega_{0}}\left(1-\beta^{*} \cos \theta\right)-i x \sin t} d t\right|^{2}$,

where $x=\frac{K \omega}{\gamma \omega_{0}} \sin \theta$.

Using the generating function for the Bessel

functions,

$$
e^{z / 2\left(t-\frac{1}{t}\right)}=\sum_{n=-\infty}^{+\infty} t^{n} J_{n}(z)
$$

and substituting $e^{i \theta}$ for $t$, we get

$$
e^{+i z \sin \theta}=\sum_{n} e^{i n \theta} J_{n}(z)
$$

and

$$
e^{-i z \sin \theta}=\sum_{n}(-1)^{n} e^{i n \theta} J_{n}(z)
$$

Substituting into $A$, we get

$$
A=\frac{e^{2} \omega^{2} K^{2}}{16 \pi^{2} c \omega_{0}^{2} \gamma^{2}} \mid \int_{-N \pi}^{+N \pi} e^{\frac{i \omega t}{\omega_{0}}\left(1-\beta^{*} \cos \theta\right)} \sum_{n=-\infty}^{+\infty}\left(e^{i(n+1) t}-e^{i(n-1) t}\right)
$$


Rearranging the summation variabies gives

$A=\frac{e^{2} \omega^{2} K^{2}}{16 \pi^{2} \omega \omega_{0}^{2} \gamma^{2}}\left|\int_{-N \pi}^{+N \pi} e^{\frac{i \omega t}{\omega}\left(1-\beta^{*} \cos \theta\right)} \sum_{n=-\infty}^{+\infty} e^{i n t(-1)^{n}\left(J_{n-1}(x)-J_{n+1}(x)\right)} d\right|^{2}$

Using the recursion relations for $J_{n}(x)$, namely

$$
J_{n-1}(x)+J_{n+1}(x)=\frac{2 n}{x} J_{n}(x)
$$

and $J_{n-1}(x)-J_{n+1}(x)=2 J_{n}^{\prime}(x)$, and noting that

$J_{-n}(x)=(-1)^{n} J_{n}(x)$, we have

$A=\frac{e^{2} \omega^{2} K^{2}}{4 \pi^{2} \omega_{0}^{2} \gamma^{2}}\left|\int_{-N \pi}^{+N \pi} e^{\frac{i \omega t}{\omega}}\left(1-\beta^{*} \cos \theta\right) \sum_{n=-\infty}^{+\infty} e^{-i n t} J_{n}^{\prime}(x) d t\right|^{2}$

The integral. over $t$ now may be done to yield

$A=\frac{e^{2} \omega^{2} K^{2}}{\pi^{2} \omega_{0}^{2} \gamma^{2}}\left|\sum_{n=-\infty}^{+\infty} J_{n}^{\prime}(x) \frac{\sin N \pi\left(\frac{\omega}{\omega_{0}}\left(1-\beta^{*} \cos \theta\right)-n\right)^{2}}{\left(\frac{\omega}{\omega_{0}}\left(1-\beta^{*} \cos \theta\right)-n\right)}\right|^{2}$.

This expression may be evaluated numerically as is, or it may be further simplified by noting that for large values of $N$, the $\frac{\sin N \pi x}{x}$ functions will have only a small overlap, allowing us to drop the cross product terms and get 
$A=\frac{e^{2} \omega^{2} K^{2}}{\pi^{2} \omega_{0}^{2} \gamma^{2}} \sum_{n=-\infty}^{+\infty} j_{n}^{\prime 2}(x) \frac{\sin ^{2} N \pi\left(\frac{\omega}{\omega_{0}}\left(1-\beta^{*} \cos \theta\right)-m\right)}{\left(\frac{\omega}{\omega_{0}}\left(1-\beta^{*} \cos \theta\right)-m\right)^{2}}$

If we write $\omega_{1}=\frac{\omega_{0}}{1-\beta^{*} \cos \theta}$, and, notine that $\beta^{*} \approx 1-\frac{1}{2 \gamma^{*} 2}$ and that $\cos \theta \approx 1-\frac{\theta^{2}}{2}, \omega_{1}$ becomes

$$
w_{1}=\frac{2 y^{* 2} \omega_{0}}{1+y^{* 2}{ }_{\theta}^{2}}
$$

Since $\beta^{*}=\beta \sqrt{-\frac{K^{2}}{\gamma^{2}}}$, from the definition of $k$, we then

get, to the same order of approximation,

$$
\omega_{1}=\frac{2 \gamma^{2} \omega_{0}}{1+k^{2}+\gamma^{2} \theta^{2}}
$$

Going through a similar series of steps for part $B$, the final result for $\frac{d I(\omega)}{d n}$ emerges as

$$
\frac{d I(\omega)}{d \Omega}=A+B=
$$

$\frac{e^{2} \omega^{2} K^{2}}{\pi^{2} \omega_{0}^{2} \gamma^{2}} \sum_{n=-\infty}^{+\infty}\left(J_{n}^{\prime 2}(x)+\left(\frac{\gamma \theta}{K}-\underline{n}\right)^{2} J_{n}^{2}(x)\right) \frac{\sin ^{2} N \pi\left(\frac{\omega}{\omega_{1}}-n\right)}{\left(\frac{\omega}{\omega_{1}}-n\right)^{2}}$,

where $\sin \theta$ has been replaced by $\theta$ and $\beta^{*}$ has been taken to be 1. Also, $x=\frac{K \omega \theta}{\psi_{0}}$, and $\omega_{1}=\frac{2 \omega_{0} \gamma^{2}}{1+K^{2}+\gamma^{2} \theta^{2}}$. This is

the same as Eq. (10) in the text. 
To get the angular distribution of power as a function of solid angle, we must integrate over all frequencies. To make this easier, we substitute $N \pi^{2} \delta(x)$ for $\frac{\sin ^{2} N \pi x}{x^{2}}$, and get

$$
\begin{aligned}
\frac{d W}{d \sigma} & =\int_{0}^{\infty} \frac{d I(\omega)}{d n} d \omega \\
& =\int_{0}^{\infty} d \omega\left\{\frac{N e^{2} \omega_{0} K^{2}}{c} \frac{8 y^{4}}{\left(1+K^{2}+y^{2} \theta^{2}\right)^{3}} \cdot \sum\left(J_{n}^{\prime 2}\left(x_{n}\right)+\left(\frac{\gamma \theta}{K}-\frac{n}{x_{n}}\right)^{2} J_{n}^{2}\left(x_{n}\right)\right)\right.
\end{aligned}
$$

Here $x_{n}=\frac{2 K n y \theta}{1+K^{2}+\gamma^{2} \theta^{2}}$. We therefore have

$$
\frac{d W}{d n}=\frac{N e^{2} \omega_{0} K^{2}}{c} \frac{8 y^{4}}{\left(1+K^{2}+\gamma^{2} \theta^{2}\right)^{3}} \sum_{n=1}^{\infty} n^{2}\left(J_{n}^{\prime 2}\left(x_{n}\right)+\left(\frac{\gamma \theta}{K}-\frac{n}{x_{n}}\right)^{2} J_{n}^{2}\left(x_{n}\right)\right) .
$$

This is expression (25) in the text. One may also integrate over all solid angle rather than over all frequencies to get the power spectrum of the radiation. Defining two new variables $r=\frac{\omega}{2 \gamma^{2} \omega_{0}}$ and $\alpha^{2}=\gamma^{2} \theta^{2}$, and again making the substitution of $N \pi^{2} \delta(x)$ for $\frac{\sin ^{2} N \pi x}{x^{2}}$, the delta functions become 


$$
\begin{aligned}
\delta\left(\omega-n \omega_{1}\right) & =\delta\left(2 \gamma^{2} \omega_{0} r-\frac{2 \gamma^{2} \omega_{0} n}{1+K^{2}+\alpha^{2}}\right) \\
& =\frac{1+k^{2}+\alpha^{2}}{2 \gamma^{2} \omega_{0} r} \delta\left(\alpha-\left(\frac{n}{r}-1-K^{2}\right)\right) \\
& =\frac{n}{2 \gamma^{2} \omega_{0}^{2} r^{2}} \delta\left(\alpha-\alpha_{n}^{2}\right),
\end{aligned}
$$

where $\alpha_{n}^{2}=\frac{n}{r}-1-K^{2}$. So,

$$
\begin{aligned}
\frac{d I(\omega)}{d n} & =\frac{4 N e^{2} K^{2} y^{2} r}{c} \sum_{n=1}^{\infty}\left(J_{n}^{\prime 2}\left(x_{n}\right)+\left(\frac{\alpha_{n}}{K}-\frac{n}{x_{n}}\right)^{2} J_{n}^{2}\left(x_{n}\right)\right) \\
& \times \delta\left(\alpha^{2}-\alpha_{n}^{2}\right) .
\end{aligned}
$$

Here $x_{n}=2 K r_{n}$. We may now integrate over all. solid angle at fixed $\omega$ to produce 


$$
\begin{aligned}
& I(\omega)=\int_{0}^{2 \pi} d \varphi \int_{0}^{\pi} \frac{d I(\omega)}{d \Omega} \sin \theta d \theta \\
& =\frac{2 \pi}{2} \int_{0}^{\infty} \frac{d I(\omega)}{d n} \alpha d \alpha \\
& =\frac{\pi}{\gamma^{2}} \int \frac{4 N e^{2} K^{2} y^{2} x}{c} \sum_{n=1}^{\infty}\left(J_{n}^{\prime 2}\left(\begin{array}{c}
x_{n} \\
n
\end{array}\right)+\left(\frac{a_{n}}{K}-\frac{n}{x_{n}}\right)^{2} J_{n}^{2}\left(x_{n}\right)\right) \\
& \times 5\left(a^{2}-\alpha_{n}^{2}\right) d u^{2} \\
& =\frac{4 \pi N e^{2} K^{2} r}{c} \sum_{n=1}^{\infty}\left(J_{n}^{\prime 2}\left(x_{n}\right)+\left(\frac{a_{n}}{K}-\frac{n}{x_{n}}\right)^{2} J_{n}^{2}\left(x_{n}\right)\right) \theta\left(a_{n}^{2}\right) .
\end{aligned}
$$

Here $\theta\left(\alpha_{n}^{2}\right)$ is the usual step function, $x_{n}=\sqrt{\frac{n}{x}-1-k^{2}}$, and $x_{n}=2 K r u_{n}$. This is the same as expression (26) in the text.

By a similar series of substitutions we can also convolve (AI) with a normalized Gaussian distribution of viewing angles, which is equivalent to allowing the electron beam to have some angular divergence. The Gaussian convolved brightness function then would look like

$$
\left.\frac{1}{\sigma^{2}} \int_{0}^{\pi} \frac{d I(\omega)}{d \sigma}\right|_{\theta-\theta^{\prime}} e^{-\frac{\theta^{\prime 2}}{2 \sigma^{2}}} \sin \theta^{\prime} d \theta^{\prime}
$$


Making the change of variables $\alpha=\gamma \theta$ and expanding the 6-function, the Gaussian smeared brightness function becomes

$$
\begin{aligned}
& \frac{1}{\sigma_{\alpha}^{2}} \sum_{n=1}^{\infty}\left(J_{n}^{\prime 2}\left(x_{n}\right)+\left(\frac{\alpha_{n}}{K}-\frac{n}{x_{n}}\right)^{2} J_{n}^{2}\left(\begin{array}{c}
x_{n} \\
n
\end{array}\right)^{2}\right. \\
& \quad \times\left(\frac{\alpha-\alpha_{n}}{\alpha_{n}} e^{-\frac{\left(\alpha-\alpha_{n}\right)^{2}}{2 \sigma_{\alpha}^{2}}} \theta\left(\alpha-\alpha_{n}\right)+\frac{\alpha+\alpha_{n}}{\alpha_{n}} e^{\left.-\frac{\left(\alpha+\alpha_{n}\right)^{2}}{2 o_{\alpha}^{2}} \theta\left(\alpha+\alpha_{n}\right)\right)}\right. \\
& \quad \times \theta\left(\alpha_{n}^{2}\right) .
\end{aligned}
$$

Here $\sigma_{\alpha}=\sigma \gamma$ and the $\theta$-functions are step functions as before. With $\alpha=0$ (on axis) expression (23) of the text results. 
1. H. Winick and S. St. Lorant, Stanford Synchrotron Radiation Project Report SSRP 75/06, (1975).

2. E. M. Purcel1, Production of Synchrotron Radiation by Wiggler Magnets, (1972), unpublished.

3. J. Madey, et al., submitted to Phys. Rev. Letters.

4. W. R. Smythe, Static and Dynamic Electricity, p. 277, McGraw-H111, (1950).

5. J. D. Jackson, Classical Electrodynamics, p. 480, John Wiley \& Sons, (1965).

6. K. Green and R. Chasman, private communication.

7. M. Sands, The Physics of Electron Storage Rings, Stanford Linear Accelerator Center Report SLAC-121, (1970)

8. R. A. Mack, Spectral and Angular Distributions of Synchrotron Radiation, Cambridge Electron Accelerator Report CEAL-1027, (1966).

9. J. Madey, private communication.

10. W. A. Littie, J. Appl. Phys. 43, 2901, (1972). 


\section{FIGURE CAPIIONS}

Figure 1. Schematic of double helix bifilar wound magnet showing alternate wires with opposite currents.

Figure 2. Polar plot of power versus angle for synchrotron radiation emitted by electron in helical orbit for various values of the magnetic f'ield parameter, $K$. The $K=.5$ case shows the behavior for small magnetic tields and the $K=$ ? case shows the strong field case.

Figure 3. Schematic representation of radiation produced in strong and weak field cases viewed both in the lab and in the moving frame.

Figure 4. Helical orbit of electron in helical magnetic field, showing angles and other parameters used in the calculation in the text.

Figure 5. Comparison of the peak spectral brightness of a helical and a conventional wiggler with the brightness spectrum of a bending magnet source for electron energies of $.5,1.0,2.0$, and $4.0 \mathrm{GeV}$. B4.0 means that the line on the curve represents a bending magnet for 4.0 GeV electron energy. The rest of the figure is explained in the text. 
Figure 6. Comparison of on axis brightness spectrum for helical wiggler as a function of frequency for two different electron beam divergences. Here $r=\omega / 2 \gamma^{2} \omega_{0}$ is the normalized frequency and $\sigma$ is the standard deviation of the electron beam's angular distribution in units of $1 / \gamma$.

Figure 7. Angular distribution of power (integrated over all frequencies) for several values of the magnetic field parameter $K$. The horizontal axis is the polar angle $\alpha=\gamma \theta$.

Figure 8. Comparison of peak values of power spectrum (Integrated over all angles) for helical and normal wigglers with power spectra for bending magnet for electron energies of .5, 1.0, 2.0, and $4.0 \mathrm{GeV}$. The vertical scale units are watts/ev/ma/cm of electron beam. The same limits and labeling are used as in Fig. 5.

Figure 9. Comparison of power spectra for helical wiggler for several values of $K$. The vertical scale is linear and the horizontal scale is the same normalized frequency variable as in Fig. 6. Figure 10. Comparison of power spectra f'or the three devices assuming reasonable parameter values for electron energies of $1.0 \mathrm{GeV}$ and $4.0 \mathrm{GeV}$. The parameters used are discussed in the text. 
$3-F-3$

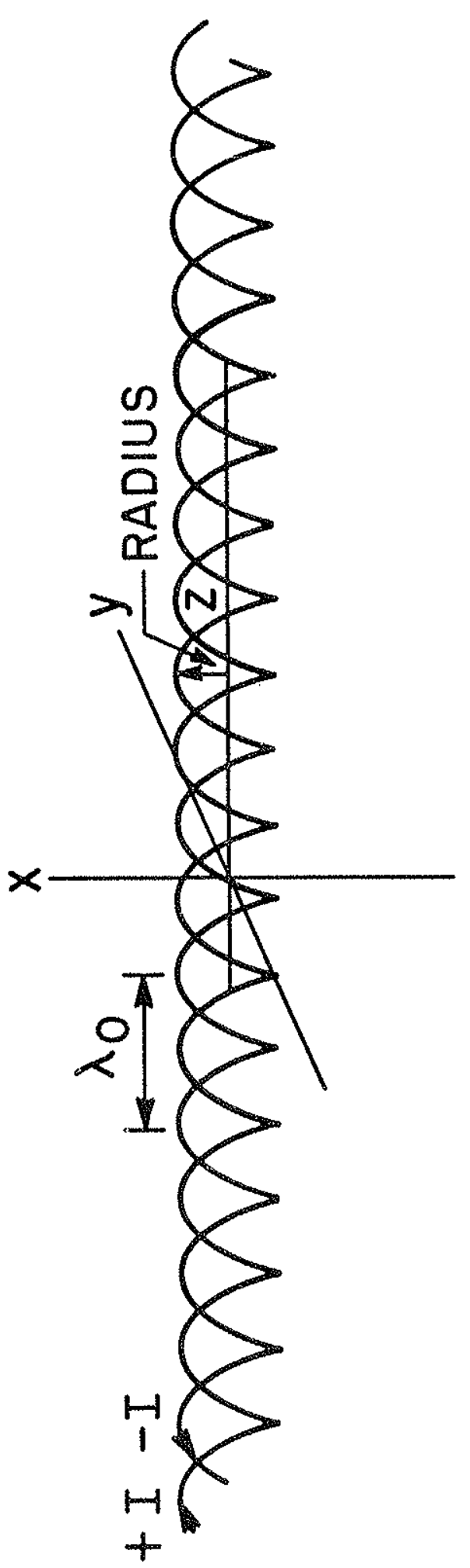

FIG. 1 


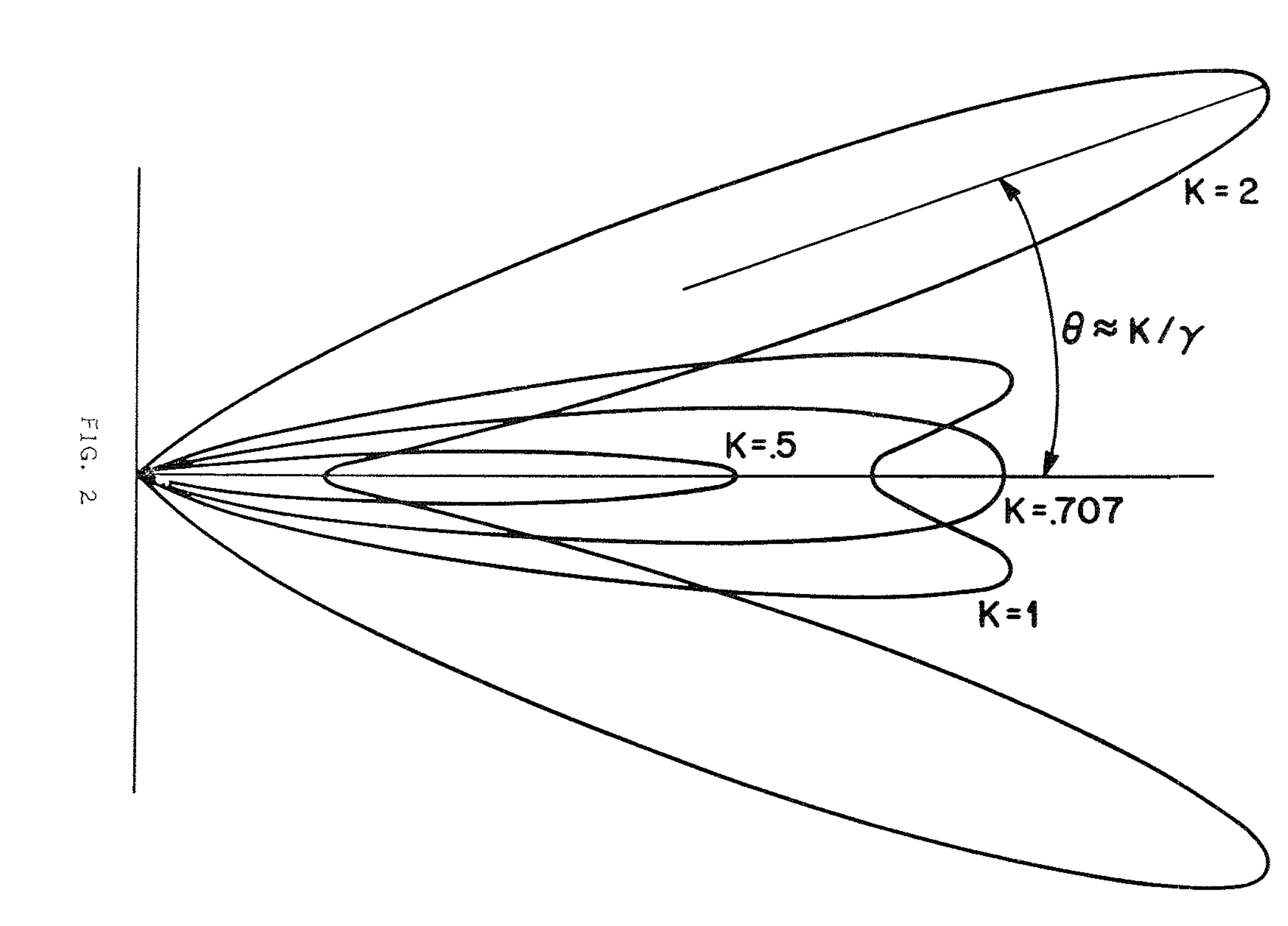



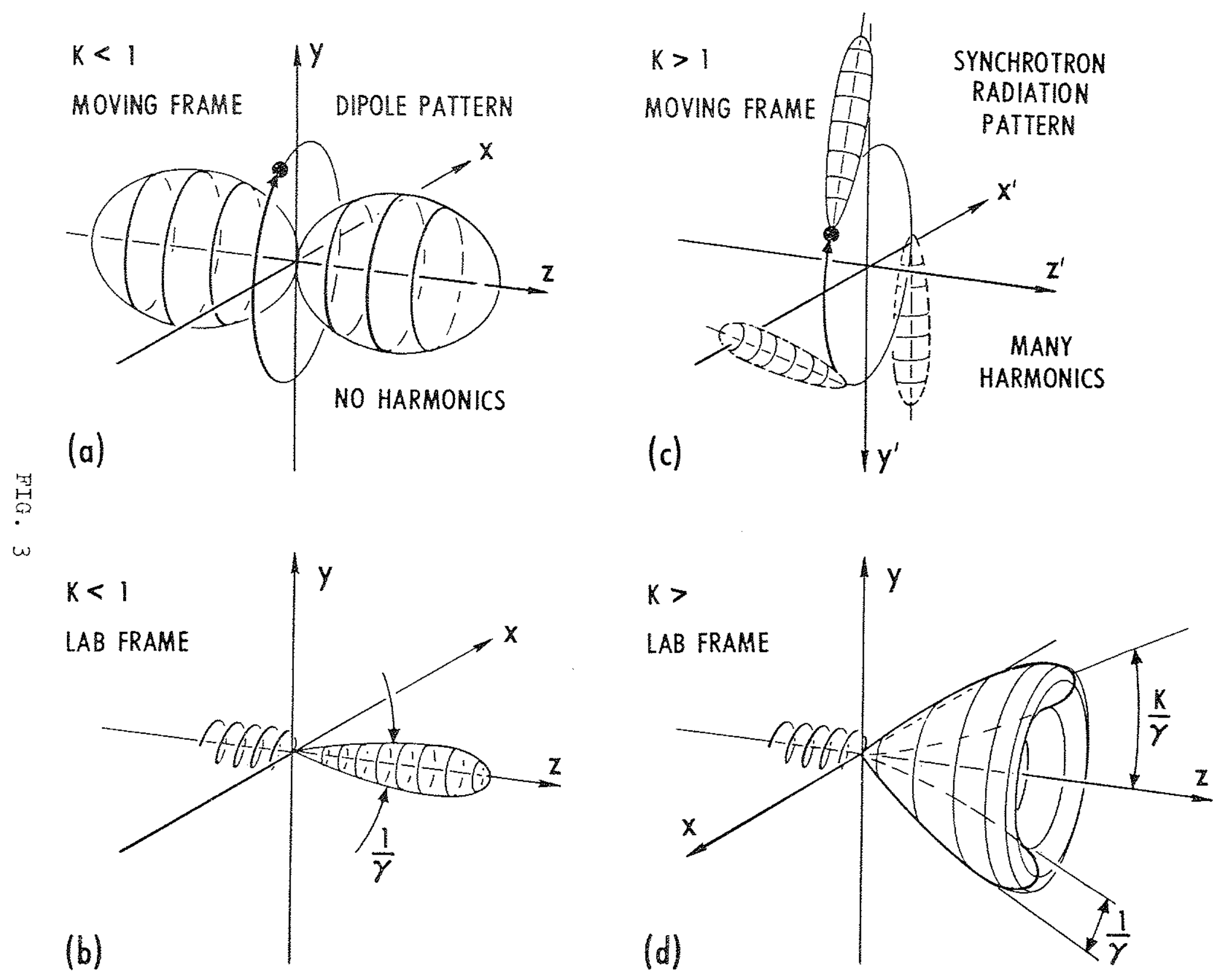


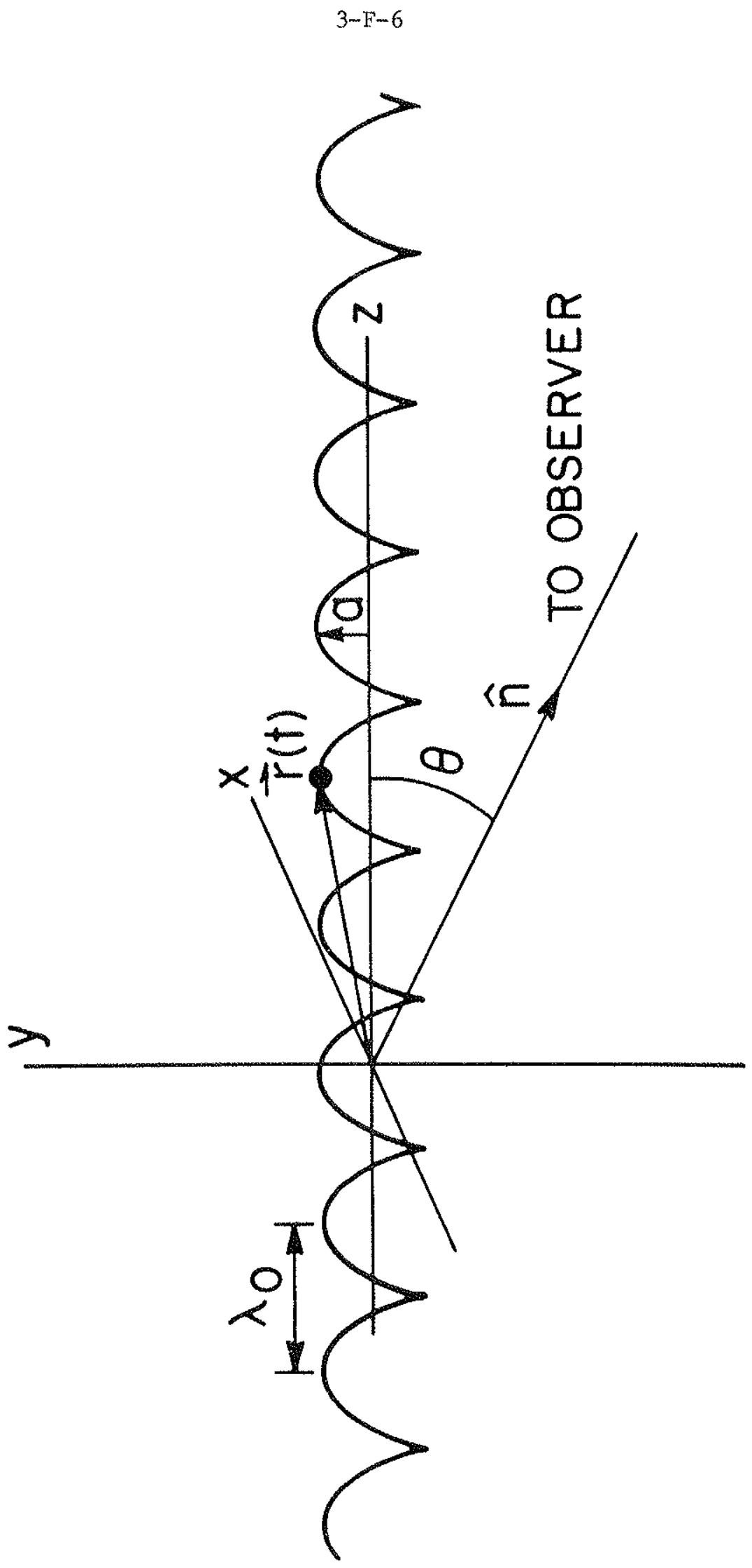

FIG. 4 


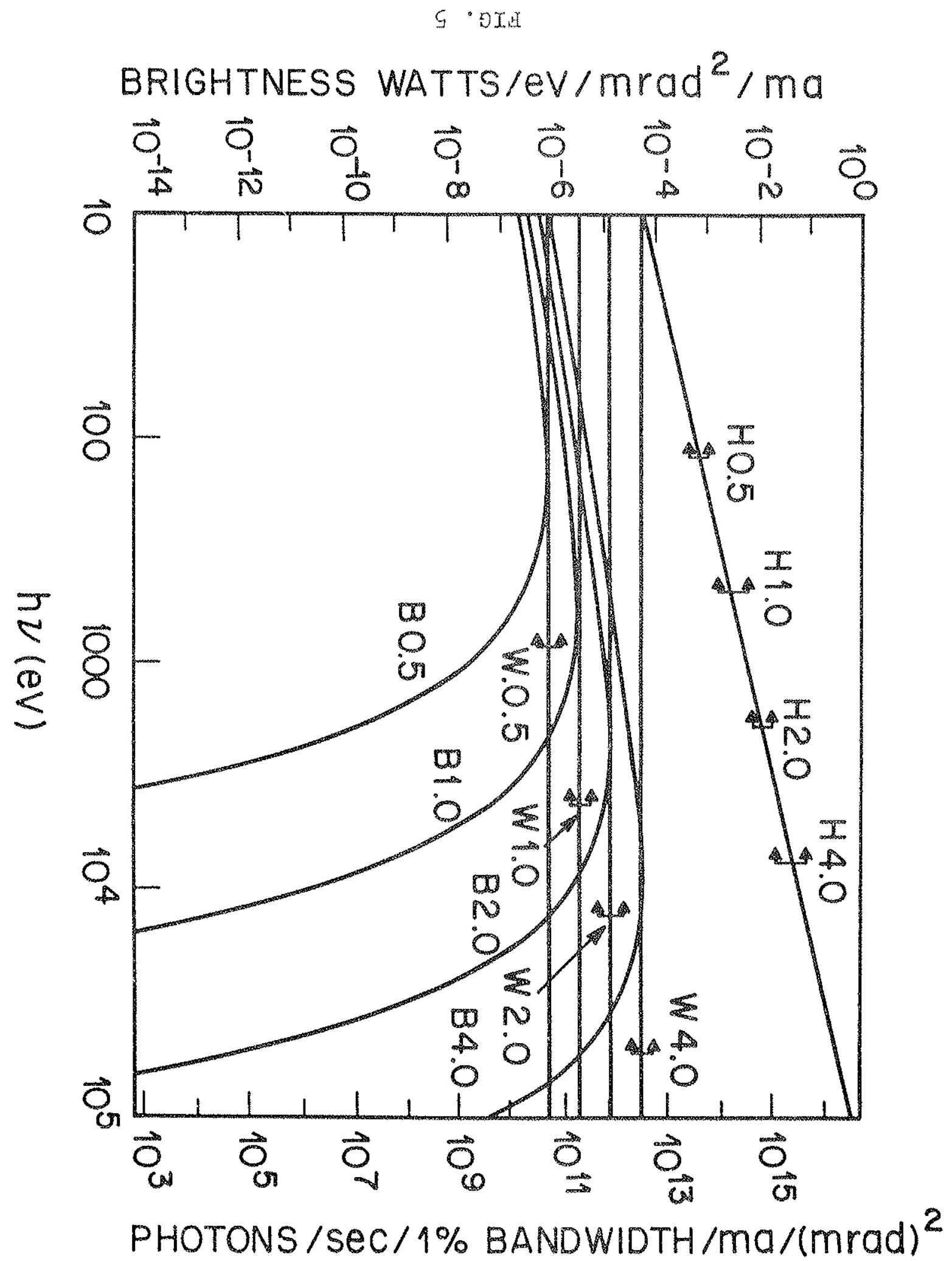




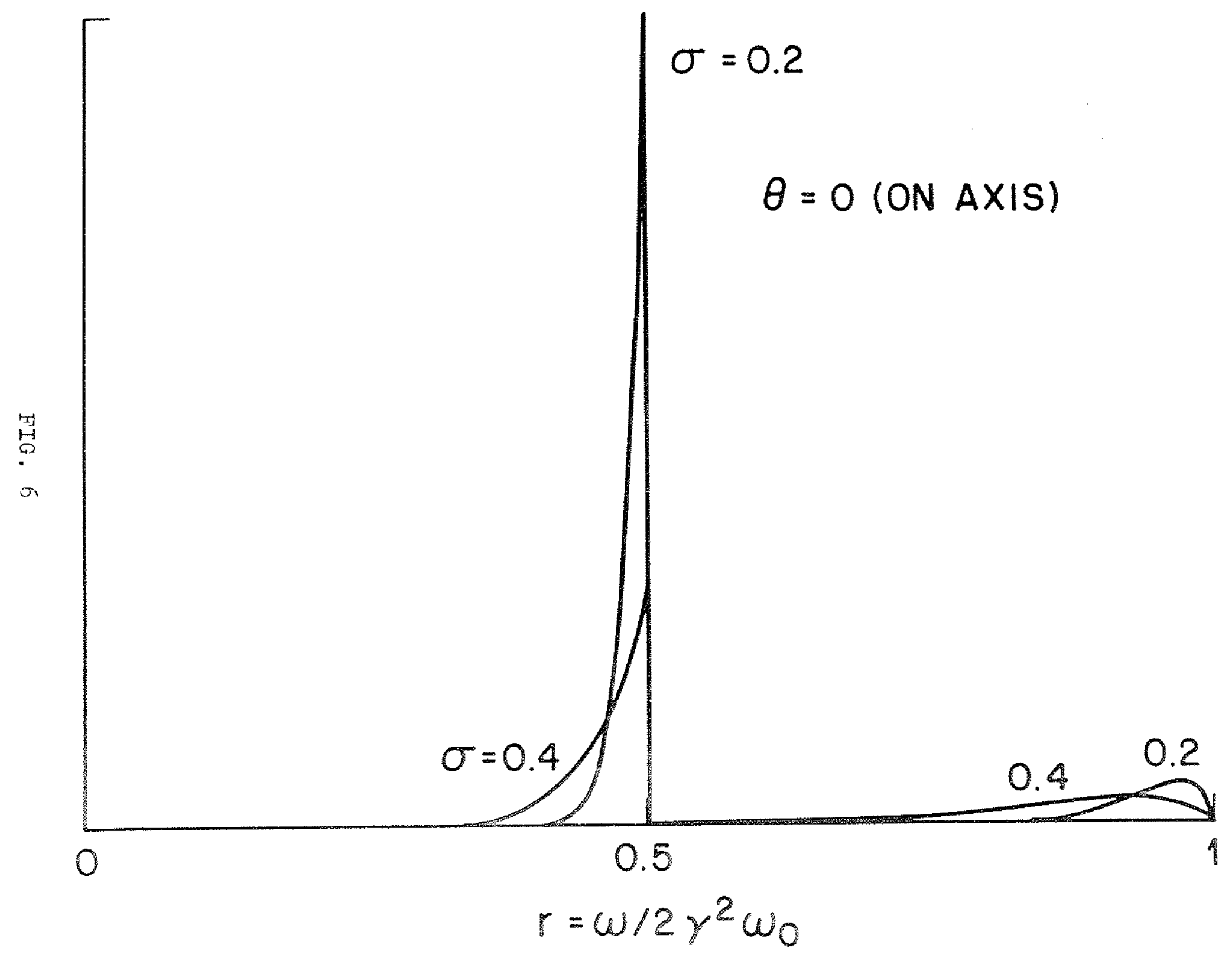




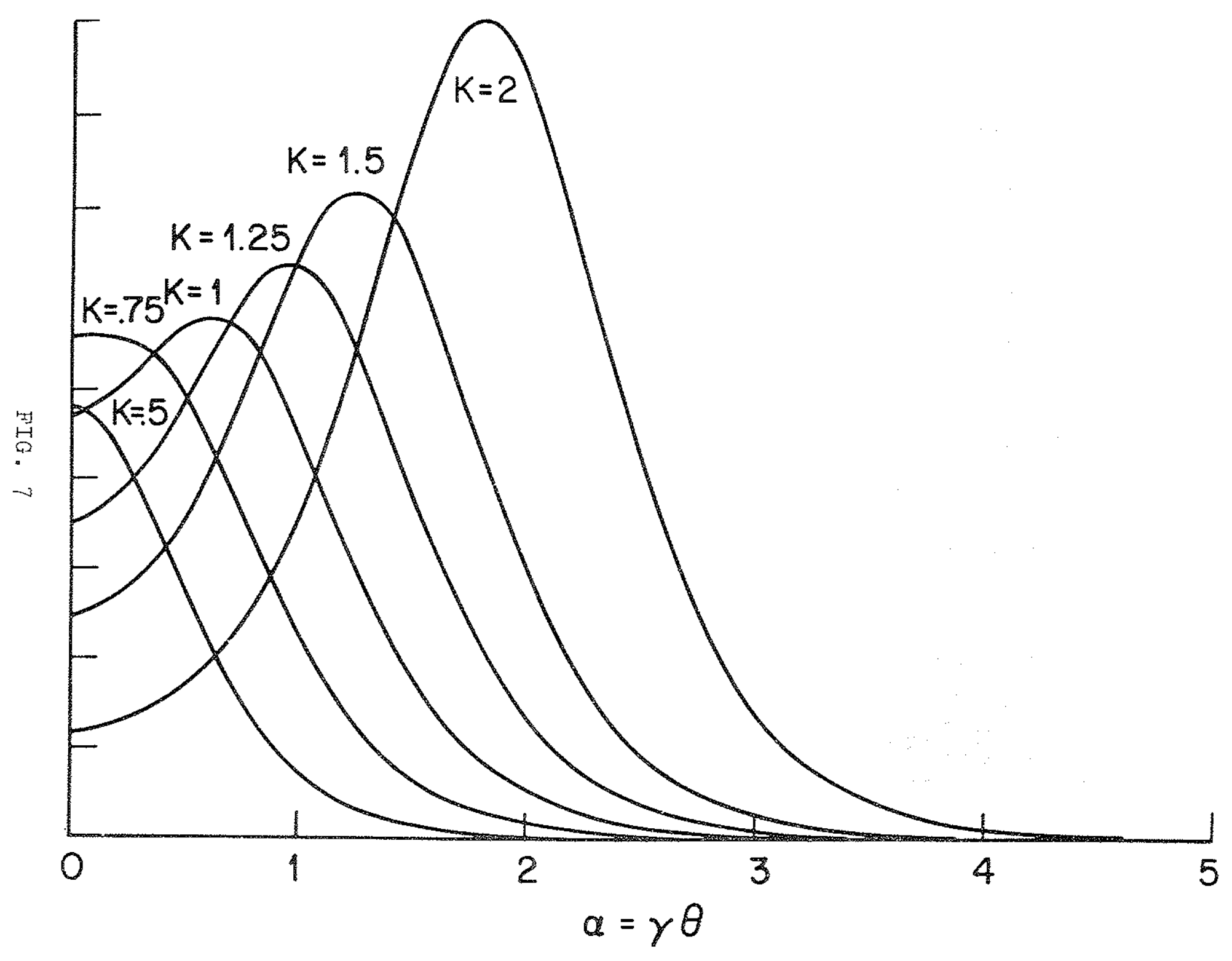


8. DI回

POWER SPECTRUM WATTS/eV/ma/cm OF ARC

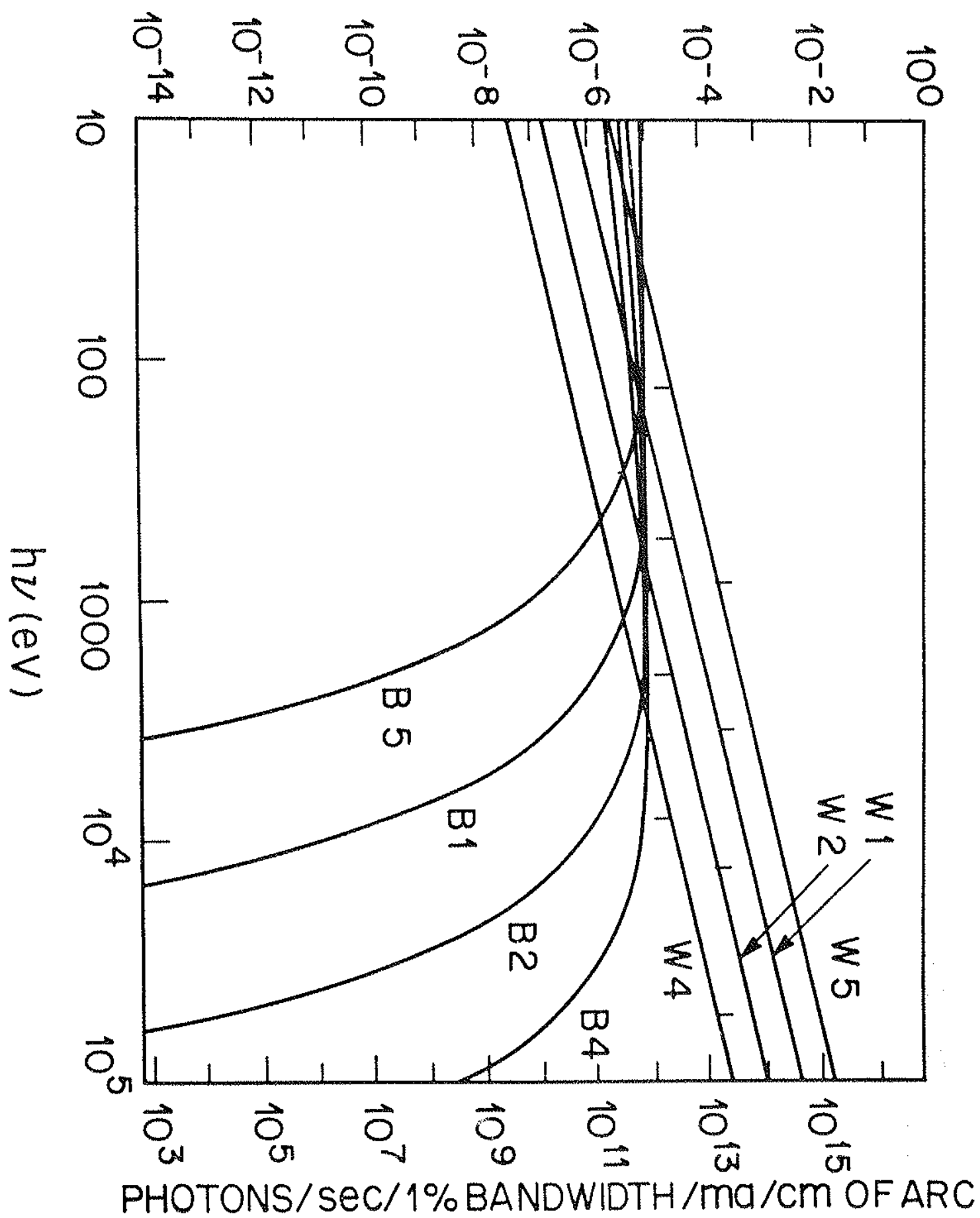




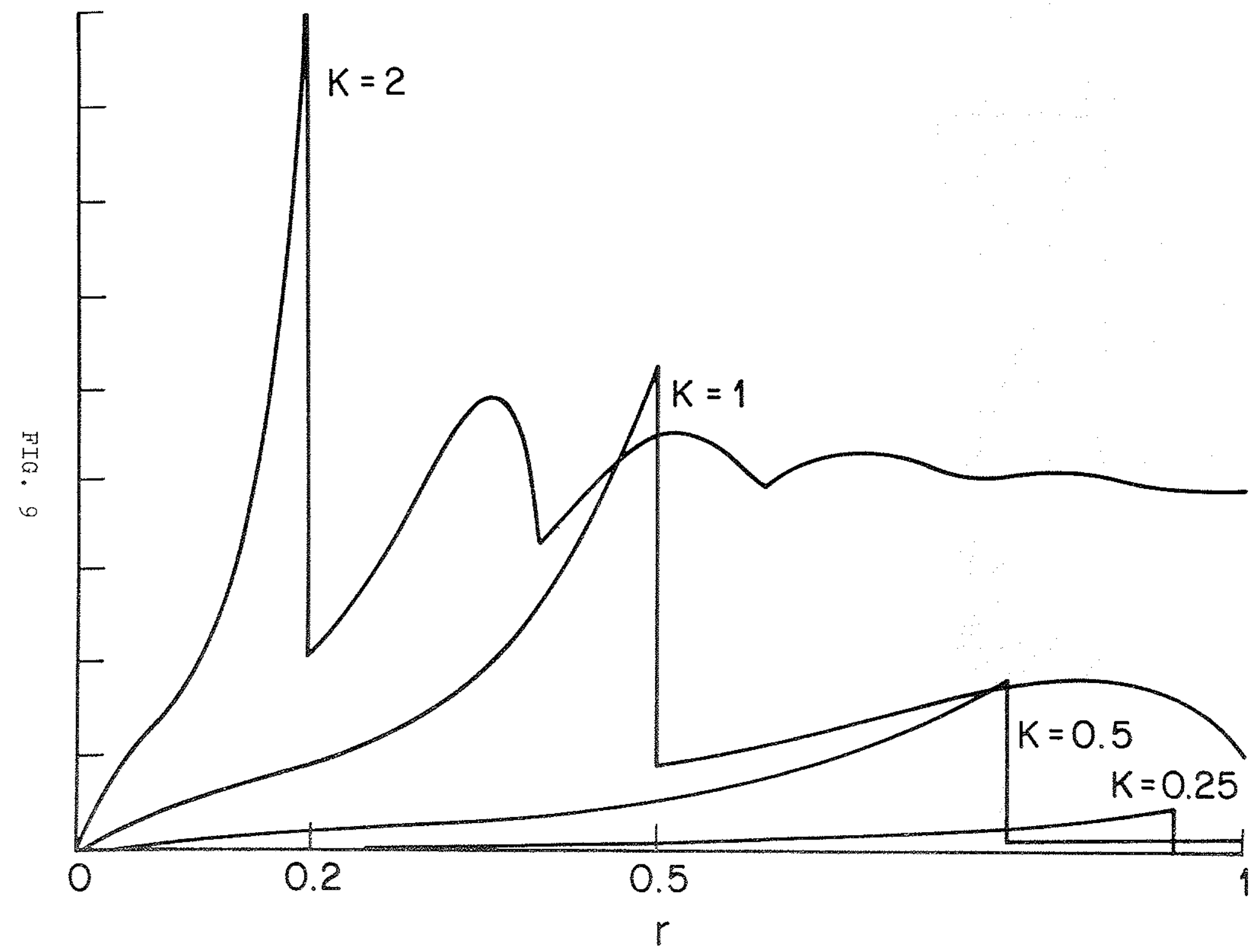

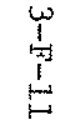




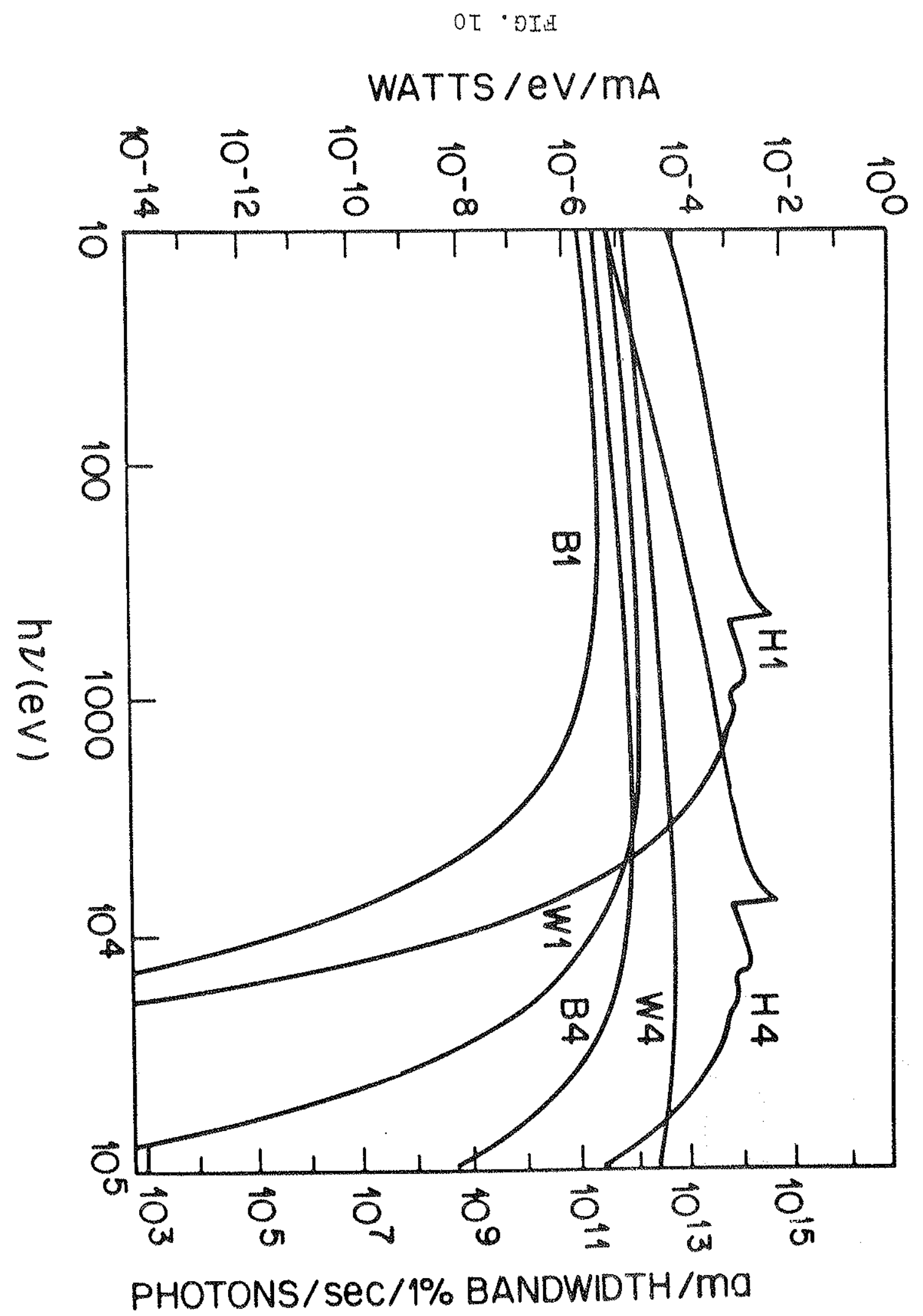


Section 4.

Submitted to the Journal of Applied Physics

\author{
ORBITS AND FTELDS IN THE HELICAL WIGGLER * \\ John P. Blewett and R. Chasman \\ Brookhaven National Laboratory, Upton, N.Y. 11973
}

\begin{abstract}
The "helical wiggler" is a device in which relativistic electrons pass through a transverse magnetic field whose direction revolves with distance along the beam axis. In this paper we discuss the electron orbits in this device. The field patterns and necessary current distributions are established. Finally, the question is treated as to whether this device can be incorporated into a storage ring without destroying the circulating beam. It is concluded that there is reason to expect satisfactory performance from helical wigglers in storage rings.
\end{abstract}

*Work performed under the auspices of the Energy Research and Development Administration. 
Introduction

In a recent publication in physical Review Letters elias et al. ${ }^{1}$ of Stanford describe the "Observation of Stimulated Emission of Radiation by Relativistic Electrons in a Spatially Periodic Transverse Magnetic Field." The observed radiation is a phenomenon predicted several years ago by Motz, ${ }^{2}$ and analyzed by E.M. Purcell (in unpublished reports) and by Madey. ${ }^{3}$ The earlier papers considered only radiation induced on passage of electrons through a magnetic field whose direction is periodically reversed. Madey's paper and the latest stanford letter describe radiation induced in a transverse magnetic field whose direction revolves around the beam axis. The experiment described in Ref. 1 used a linear accelerator beam which made a single passage through the device. The possibility of incorporation of a spiralling transverse magnetic field in a storage ring to yield a radiation spectrum more sharply peaked than the usual synchrotron radiation spectrum has recently been recognized by Kincaid." His paper "A Short Period helical Wigglex as an Improved source of synchrotron Radiation" appears as a companion paper to this one.

The radiation under discussion is essentially synchrotron radiation emitted when electrons travel on a helical path through a spiralling transverse magnetic field. When the electron orbit makes many turns in the spiralling field the radiation spectrum, heavily affected by Lorentz iransformations, peaks at a wavelength roughly $y^{-2}$ times the period of the spiralling magnetic field. In the stanford study the period of the spiralling field was $3.2 \mathrm{~cm}$. Electrons of energy $24 \mathrm{MeV}$ passed through the field pattern which was applied over a distance of $5.2 \mathrm{~m}$ along the electron orbit. The transverse field on axis was $2300 \mathrm{G}$. Radiation was observed at the predicted frequency of $1.0 .6 \mu$. 
Since the radiation in this system is much more nearly monochromatic than the radiation from electrons in a storage ring or synchrotron, it is of considerable interest to consider inclusion of a spiralling transverse field system in a storage ring built as a dedicated source of synchrotron radiation. In this paper the field patterns and electron orbits in the spiralling field system are explored and the implications for incorporating it in a storage ring are derived.

At Stanford, the device is referred to as a "free electron laser." Synchrotron radiation specialists describe it as a "helical wiggler."

\section{Description of Magnet System}

The spiralling field pattern is produced by a double helix bifilar magnet which can be visualized if one assumes that, on the outside of a bore tube an open helix is wound and then a second helix is wound in the spaces between turns of the first helix. When currents in opposite directions are passed through the two helices the central, axial magnetic field is cancelled and the spiralling transverse field pattern appears.

If the helices consist of wires of infinitesimal cross section, the field pattern on axis can be derived as a function of current in the helices. The result for a single, open helix is given by Smythe 5 and is derived for the double helix by kincaid. ${ }^{4}$

\section{Field and Current Distributions}

It will be assumed that the field pattern has a sinusoidal variation in the axial direction and that no higher harmonics are present. 


$$
4-4
$$

The magnetic field patterns and current distributions in the magnet system are derived in the Appendix. The fields to be traversed by the electron beam are given by:

$$
\begin{aligned}
& B_{r}=2 B_{o}\left(I_{0}(k r)-\frac{i}{k r} I_{1}(k r)\right) \sin (\theta-k z) \\
& B_{\theta}=\frac{2 B_{0}}{k r} I_{1}(k x) \cos (\theta-k z) \\
& B_{z}=-2 B_{0} I_{1}(k r) \cos (\theta-k z),
\end{aligned}
$$

where $B_{0}$ is the transverse field amplitude at the axis of the system, $I_{0}$ and $I_{1}$ are Besse1 functions,

$k=2 \pi / \lambda_{0}$, where $\lambda_{0}$ is the pitch of the helical winding.

Near the axis the Bessel functions can be approximated by expressions given in the Appendix.

The current in the helical winding required to produce a transverse field $B_{0}$ on the axis of a helix of pitch $\lambda_{0}$ is plotted as a function of the ratio of helix radius to pitch in Fig. 1.

\section{E1ectron Orbits}

The analysis which follows has been guided by computer runs which have indicated the character of the orbits. The computer runs have traced electrons under various conditions through the fields described by Eqs. (1). It has thus been established that the orbit includes oscillations at three main frequencies. The primary motion is helical with an orbit radius of a small fraction of a millimeter. The period of the motion is the same as that of the transverse field and its axis oscillates around the physical axis of the helix with a much lower frequency. At a still lower frequency the $x$ and $y$ components of the intermediate frequency oscillation couple to each other and exchange energy. 
The reasons for this behavior and the important parameters can be established by an approximate solution of the equations of motion.

An important simplification is possible when it is realized that excursions from the axis are small and that the major velocity component is the paraxial one. Since all applications will use highly relativistic electrons it is legitimate to set $\dot{z}=c$ and $z=c t$. This reduces to two the number of equations of motion which must be solved.

First, we shall establish the radius $r_{0}$ of the helical orbit. The field patterns (1), for the regions close to the axis can be approximated by

$$
\begin{aligned}
& B_{r}=B_{0} \sin (\theta-k c t) \\
& B_{\theta}=B_{0} \cos (\theta-k c t) \\
& B_{z}=0 .
\end{aligned}
$$

It is easy to show that the helical orbit through these fields has

$$
\begin{aligned}
& \dot{\theta}=\mathrm{kc} \\
& r=r_{0}=1 /\left(\mathrm{k}^{2} \rho\right),
\end{aligned}
$$

where $\rho\left(=\frac{m_{0} \gamma c}{e B_{0}}\right)$ is the cyclotron radius in the field $B_{0}$.

When the lower frequency oscillation has carried the helical orbit some distance from the axis, the amplitudes of ${ }^{B}{ }_{r}$ and ${ }_{B}$, given by (1), are no longer equal and the projection of the helical orbit on the $r, \theta$ plane is no longer exactly circular. Its average value will, however, be given by solution of the following equation (expressed in a coordinate system having its origin on the instantaneous axis of the helix)

$$
m r \dot{\theta}^{2}=-\operatorname{er} \dot{\theta} B_{z}+e \dot{z} B_{t},
$$

where $B_{t}$ represents the transverse field which will alternate between ${ }_{r}$ and ${ }^{B}{ }_{\theta}$ (of the original coordinate system). From (1) its average value will be 
$B_{0} I_{0}(k R)$, where $R$ is the displacement of the instantaneous axis of the helix from the central axis of the wiggler. Now, setting $\dot{\theta}$ again equal to $k c$ and neglecting the small contribution of the $B_{z}$ term in (3), we obtain

$$
r_{0}=I_{0}(k R) /\left(k^{2} \rho\right)
$$

Using the parameters of the Stanford experiment $\left(k=196 \mathrm{~m}^{-1}, \rho=0.348 \mathrm{~m}\right)$, we find for $R=0, r_{0}=0.075 \mathrm{~mm}$. As will be shown later, the stanford parameters with no initial correction lead to a "betatron oscillation" with an amplitude of about $6 \mathrm{~mm}$. At the maximum excursion in this oscillation the value of $\mathrm{r}_{\mathrm{o}}$ is about $0.10 \mathrm{~mm}$. These figures are in good agreement with those obtained from the computer runs.

To derive the characteristics of the lower frequency oscillations it is necessary to solve the equations of motion

$$
\begin{aligned}
& \ddot{x}=(e / m)\left(\dot{y}_{z}-c B_{y}\right) \\
& \ddot{y}=(e / m)\left(c B_{x}-\dot{x}_{z}\right)
\end{aligned}
$$

We substitute for $B_{x}, B_{y}$ and $B_{z}$ the expressions ( $A 3$ ) and we make the following substitution for $x$ and $y$ :

$$
\begin{aligned}
& x=r_{0} \cos k c t+u \\
& y=r_{0} \sin k c t+v .
\end{aligned}
$$

In making the substitution we note the fact that $\mathrm{kr}_{\mathrm{o}}$ is of the order of 0.01 when the Stanford parameters are used and is even smaller for higher energy electrons. Accordingly, it is legitimate to neglect $\mathrm{k}^{2} \mathrm{r}_{0}^{2}$ with respect to unity. Assuming that $\mathrm{u}$ and $\mathrm{v}$ are slowly varying compared to $\cos \mathrm{kct}$, one can write from (5) and (6): 


$$
\begin{aligned}
& \ddot{u}+2 \delta \dot{v}+\omega^{2} u=0 \\
& \ddot{v}-2 \delta \dot{u}+\omega^{2} v=0,
\end{aligned}
$$

where $\delta=\frac{k c r_{o}}{2 p}\left(1+\frac{k^{2}}{4}\left(u^{2}+v^{2}\right)\right)$

$$
\omega^{2}=\frac{k^{2} c^{2} x_{o}}{2 \rho}\left(1+\frac{k^{2}}{8}\left(u^{2}+v^{2}\right)\right) \text {. }
$$

Solving Eqs. (7), taking into account the fact that $\delta$ is small compared with $w$, we find that $u$ and $v$ are combinations of trigonometric functions of $(\omega \pm \delta)$. The coefficients of the functions will be determined by initial conditions. The values of $u$ and $v$ are:

$$
\begin{aligned}
& u=\left(x_{0}-r_{0}\right) \cos \omega t \cos \delta t+\frac{1}{\omega}\left(\delta x_{0}-v_{y}+r_{o}(k c-\delta)\right) \sin \omega t \sin \delta t \\
&+\frac{1}{\omega}\left(\delta y_{0}+v_{x}\right) \sin \omega t \cos \delta t-y_{0} \cos \omega t \sin \delta t \\
& v=\frac{1}{\omega}\left(v_{y}-\delta x_{0}-r_{0}(k c-\delta)\right) \sin \omega t \cos \delta t+\left(x_{0}-r_{0}\right) \cos \omega t \sin \delta t \\
& \quad+y_{0} \cos \omega t \cos \delta t+\frac{1}{\omega}\left(\delta y_{0}+v_{x}\right) \sin \omega t \sin \delta t
\end{aligned}
$$

where $x_{0}, y_{0}, v_{x}$ and $v_{y}$ are the initial values of $x, y, \dot{x}$ and $\dot{y}$. Using the Stanford parameters we find that $\delta$ is of the ordex of $1 \%$ of $w$ which, in turn, is of the order of $1 \%$ of $\mathrm{kc}$. Neglecting small terms and setting all of the initial parameters equal to zero we obtain: 


$$
\begin{aligned}
& x=r_{0}(t) \cos k c t+\left(k c r_{0}(t=0) / \omega\right) \sin \omega t \sin \delta t \\
& y=r_{0}(t) \sin k c t-\left(k c r_{0}(t=0) / \omega\right) \sin \omega t \cos \delta t .
\end{aligned}
$$

For zero intitial conditions, there will be a "betatron oscillation" with an amplitude of the order of one hundred times $r_{0}$, the radius of the basic helical motion and with a frequency of the order of $1 \%$ of that of the basic helical motion.

We turn now to establishing the values of the lower oscillation frequencies. First we use (4) to establish an average value of $r_{0}$. From (6), (9) and (10)

$$
\begin{aligned}
\mathrm{R}^{2} & \approx u^{2}+v^{2} \quad \text { (assuming } u \text { and } / o r v \text { is much larger than } r_{o} \text { ) } \\
& =R_{0}^{2} \sin ^{2}(\omega t+\varphi),
\end{aligned}
$$

where $R_{0}$ is the maximum excursion at the intermediate frequency. $R_{0}$ and $\varphi$ are determined by the initial conditions .

From (4):

$$
r_{0} \simeq \frac{1}{k^{2} p}\left(1+\frac{k^{2} R_{0}^{2} \sin ^{2}(\omega t+\varphi)}{4}\right)
$$

Hence the average value of $r_{0}$ is

$$
r_{o, a v}=\frac{1}{k^{2} p}\left(1+\frac{k^{2} R_{o}^{2}}{8}\right)
$$

Applying the same procedures to (10):

$$
w^{2}=\frac{c^{2}}{2 p^{2}}\left(1+\frac{k^{2} \mathrm{R}_{o}^{2}}{8}\right)\left(1+\frac{k^{2} \mathrm{R}_{o}^{2}}{16}\right)
$$


and

$$
\begin{aligned}
& \omega=\frac{0.707 c}{\rho}\left(1+\frac{3 k^{2} R_{0}^{2}}{32}\right) \\
& \delta=\frac{c}{2 k \rho^{2}}\left(1+\frac{k^{2} R_{o}^{2}}{4}\right)
\end{aligned}
$$

Inserting the Stanford parameters, after a few algebraic manipulations the parameters prove to be

$$
\begin{aligned}
& R_{o}=6.0 \times 10^{-3} \mathrm{~m} \\
& \omega=7.4 \times 10^{8} \mathrm{sec}^{-1} \\
& \delta=1.0 \times 10^{7} \mathrm{sec}^{-1}
\end{aligned}
$$

For comparison $k c=5.9 \times 10^{10} \mathrm{sec}^{-1}=80 \mathrm{\omega}$.

The results of the computer runs using the correct field expressions (1) are shown in Figs. 2 and 3 for the case of zero initial conditions. Figure 2 shows the first 100 periods of the helical oscillation; Fig. 3 shows the behavior of $u$ and $v$ over an impractically long wiggler having over 700 periods. The results are in good agreement with the approximate theory out 1 ined above. The value of $\omega$ is $7.6 \times 10^{8}$ to be compared with the predicted value of $7.4 \times 10^{8}$. The predicted value for the oscillation amplitude was $6.0 \mathrm{~mm}$, to be compared with the correct value of $6.2 \mathrm{~mm}$. The hypothetical computed wiggler was not long enough to establish the value of $\delta$ but evidently the predicted value is of the right order of magnitude.

\section{Effect of a Superposed Axial Field}

In the Stanford experiment a 1000-G axial field was superposed on the wiggler field pattern by addition of a solenoid which enclosed the wiggler. Such a field can have marked effects both on the helical motion and on the betatron oscillation frequency and amplitude. 
The wavelength of the helical motion will remain the same as the axial period of the helical winding. But the value of $x_{0}$, obtained from (3), will change if the axial field is strong enough that the first term on the right of (3) no longer can be neglected. Equation (3) then yields

$$
r_{0}=\frac{I_{0}(k r)}{k^{2} \rho\left(1 \pm \frac{1}{k \rho_{1}}\right)}
$$

where $p_{1}\left(=\frac{m c}{e B_{1}}\right)$ is the cyclotron radius in the added axia1 field $B_{1}$. In a field of $10 \mathrm{kG}$ the added term will make a change of about $6 \%$ in the value of $\mathrm{r}_{0}$. Whether $r_{0}$ is increased or decreased depends on the direction of the applied field.

To find the effect of the applied field on the betatron oscillations we re-examine Eqs. (7) and (8). Equation (7) will have the same form but it will be found that the expression for $\delta$ is changed to

$$
\delta=\frac{k c r_{o}}{2 p}\left(1+\frac{k^{2}}{4}\left(u^{2}+v^{2}\right)\right) \pm \frac{c}{2 p_{1}}
$$

Here, as before, the choice of plus or minus sign depends on the direction of the applied field. If the applied field $B_{1}$ is of the same order as $B_{0}$, then, since $\mathrm{kr}_{0}$ is small (of the order of $10^{-2}$ ) the new term is the only significant one. The expression for $\omega^{2}$ is unchanged but now $\delta$ is of the same order as $w$ and terms in $\delta / \omega$ no longer can be neglected. Equations (7) now yield for the frequency of the betatron oscillation the quantity

$$
\sqrt{\omega^{2}+2 \delta^{2} \pm 2 \delta \sqrt{\omega^{2}+\delta^{2}}} .
$$

For small amplitude betatron oscillations where $k^{2} u^{2}$ and $k^{2} v^{2}$ are negligible compared with unity, (15) becomes 


$$
k c \sqrt{\frac{1}{2}\left(\frac{r_{o}}{\rho}+\frac{1}{k^{2} \rho_{1}^{2}}\right) \pm \frac{1}{k \rho_{1}} \sqrt{\frac{1}{2}\left(\frac{r_{o}}{\rho}+\frac{1}{2 k^{2} \rho_{1}^{2}}\right)}}
$$

Por applied axial fields of 1,5 and $10 \mathrm{~kg}$ the two frequencies $\mathrm{f}_{1}$ and $\mathrm{f}_{2}$ given by (16) are (using the Stanford parameters):

$\begin{array}{ccc}\frac{B_{1}(k G)}{1} & \frac{f_{1}\left(\mathrm{sec}^{-1}\right)}{8.2 \times 10^{8}} & \frac{f_{2}\left(\mathrm{sec}^{-1}\right)}{1} \\ 5 & 20.5 \times 10^{8} & 4.5 \times 10^{8} \\ 10 & 38.4 \times 10^{8} & 1.8 \times 10^{8} \\ & & 1.0 \times 10^{8}\end{array}$

The oscillation no longer has the same character as that produced only by the wiggler but is the sum of sines and cosines of the two frequencies with amplitudes determined by initial conditions. The overall oscillation amplitudes are smaller than before and can be reduced to negligible levels by suitable choices of initial conditions.

\section{Practical Considerations}

Several practical problems remain to be solved before the helical wiggler can be incorporated into a storage ring.

First, a reasonable end configuration must be designed and the end field pattern studied. During our computer studies we have introduced a tapered field pattern at the end, maintaining the same period of the helical field pattern. Orbits through this pattern were not notably different from the orbits in the wiggler with discontinuous ends. It appears impractical to taper the end fields so slowly that the entry and exit behavior can be considered adiabatic. 
Second, such large oscillations as those predicted for zero initial conditions cannot be tolerated. Magnetic kicks must be introduced at the entrance to reduce the betatron oscillations to negligible amplitudes and at the exit to restore the electrons to the equilibrium orbit of the storage $r i n g$. Such kicks have been introduced in the computer program; as predicted by Eq. (12) an initial $\mathrm{v}_{\mathrm{y}}$ of approximately $\mathrm{kcr}_{\mathrm{o}}$ is required. This is entirely effective in reducing the radial excursions to fractions of a millimeter. The initial deflection required for the parameters considered was about 20 mrad. Figure 4 shows the computed orbits with such a kick. The kick given was that predicted by the approximate theory presented above. In the orbits shown in Fig. 4 a small betatron oscillation remains in the y plane. Exact cancellation in both planes can be achieved by trial and error.

Third, wiggler parameters should be established for use at the higher electron energies contemplated for use in synchrotron radiation facilities. To obtain $x$-rays of the highest possible energy it will be necessary to use a helix with as short a pitch as possible. But the graph of Fig. I indicates that, for a pitch less than about twice the wiggler diameter, the current demands are becoming excessive. Methods for studying this problem are included in the Appendix. In particular, Eq. (A10) can be applied to establish performance on physically realizable wigglers. In practice windings probably will be chosen of sufficient thickness that the second exponential term is of the order of about one-tenth of the first. To establish limits, however, we shall assume that the coil is infinitely thick and the second exponential vanishes. Two assumptions will be tested about achievable current density in the superconducting coil. The fields at the coil windings will be high - of the order of $50 \mathrm{kG}$ for $10 \mathrm{kG}$ transverse fields at the axis - and this will limit current densities for 
the currently available niobium-titanium wires to the order of $50,000 \mathrm{~A} / \mathrm{cm}^{2}$. We will then assume that developments now in progress on stranded $\mathrm{Nb}_{3} \mathrm{Sn}$ will result soon in current densities of $1.00000 \mathrm{~A} / \mathrm{cm}^{2}$. As a reasonable minimun value for wiggler radius we choose $0.5 \mathrm{~cm}$ For these parameters, we derive from (Al0) the data given in Table $I$.

Kincaid ${ }^{4}$ has presented reasons for preferring to keep his parameter $K$ close to unity. $K$ is a dimensionless parameter which, for $B_{0}$ in gauss and $\lambda_{0}$ in centimeters, has the value $9.3 \times 10^{-5} \mathrm{~B}_{\mathrm{o}} \lambda_{\mathrm{o}}$. From Table I achievable parameters will be a pitch of about $2 \mathrm{~cm}$ and a transverse field of about $5000 \mathrm{G}$.

The cyclotron radius for 2 - GeV electrons in a field of $5000 \mathrm{G}$ is $11 \mathrm{~m}$. The radius of the helical orbit will be about one micron. The peak in the emission spectrum will be at about 13 angstrom units.

Finally, it must be shown that the effect of the wiggler is not to destroy the circulating beam in a storage ring into which it is introduced. That subject is discussed in the next section.

\section{The Helical Wiggler in a Storage Ring}

The helical wiggler must be matched optically if it is to be incorporated into a storage ring. This is necessary to maintain orbit stability in the ring. Equations (9) and (10) show that for a helical wiggler of practical length $(\delta t \ll 1)$ one can approximate the "smooth" transverse motion by 


$$
\begin{aligned}
& u=\left(x_{0}-r_{0}\right) \cos \omega t+\frac{v_{x}}{\omega} \sin \omega t \\
& v=\frac{v_{y}-r_{0} k c}{\omega} \sin \omega t+y_{0} \cos \omega t
\end{aligned}
$$

Assuming that the wiggler is displaced horizontally by the amount $r_{0}$ and that the beam is kicked in and out to give $v_{y}= \pm r_{0} k c$ the equilibrium orbit of the storage ring will be preserved. Using the nomenclature of Courant and Snyder ${ }^{6}$ the effect of the wigglex on the betatron motion can be described by simple transfer matrices:

$$
M_{x}=M_{y}=\left[\begin{array}{cc}
\cos \mu_{w} & \beta_{w} \sin \mu_{w} \\
-\gamma_{w} \sin \mu_{w} & \cos \mu_{w}
\end{array}\right],
$$

where $\mu_{w}=\omega T_{w}=\omega L_{w} / c$,

$\mathrm{T}_{\mathrm{w}}$ is the passage time through the wiggler,

$L_{w}$ is the length of the wiggler,

$\beta_{w}=L_{w} / \mu_{w}=c / \omega$,

$\gamma_{w}=1 / \beta_{w}$.

If the helical wiggler is inserted in a storage ring between two points 1 and 2 , then, for proper matching, it is required that

$$
\left[\begin{array}{l}
\alpha_{2}^{x} \\
\beta_{2}^{x} \\
\gamma_{2}^{x}
\end{array}\right]=\left[\begin{array}{ccc}
M_{11}^{x} M_{22}^{x}+M_{12}^{x} M_{21}^{x} & -M_{21}^{x} M_{11}^{x} & -M_{12}^{x} M_{22}^{x} \\
-2 M_{11}^{x} M_{12}^{x} & \left(M_{11}^{x}\right)^{2} & \left(M_{12}^{x}\right)^{2} \\
-2 M_{22}^{x} M_{21}^{x} & \left(M_{21}^{x}\right)^{2} & \left(M_{22}^{x}\right)^{2}
\end{array}\right]\left[\begin{array}{l}
\alpha_{1}^{x} \\
\beta_{1}^{x} \\
\gamma_{1}^{x}
\end{array}\right]
$$


and

$$
\left[\begin{array}{c}
\alpha_{2}^{y} \\
\beta_{2}^{y} \\
\gamma_{2}^{y}
\end{array}\right]=\left[\begin{array}{ccc}
M_{11}^{y} M_{22}^{y}+M_{12}^{y} M_{21}^{y} & -M_{21}^{y} M_{11}^{y} & -M_{12}^{y} M_{22}^{y} \\
-2 M_{11}^{y} M_{12}^{y} & \left(M_{11}^{y}\right)^{2} & \left(M_{12}^{y}\right)^{2} \\
-2 M_{22}^{y} M_{21}^{y} & \left(M_{21}^{y}\right)^{2} & \left(M_{22}^{y}\right)^{2}
\end{array}\right]\left[\begin{array}{c}
\alpha_{1}^{y} \\
\beta_{1}^{y} \\
\gamma_{1}^{y}
\end{array}\right]
$$

where the $\alpha^{\prime} s, \beta^{\prime} s$ and $\gamma^{\prime} s$ are the horizontal and vertical orbit parameters ${ }^{6}$ of the storage ring at points 1 and 2 .

Using Eqs. (19) one obtains

$$
\left[\begin{array}{c}
\alpha_{2}^{x} \\
\beta_{2}^{x} \\
\gamma_{2}^{x}
\end{array}\right]=\left[\begin{array}{ccc}
\cos 2 \mu_{w} & \frac{1}{2 \beta_{w}} \sin 2 \mu_{w} & -\frac{1}{2} \beta_{w} \sin 2 \mu_{w} \\
-\beta_{w} \sin 2 \mu_{w} & \cos ^{2} \mu_{w} & \beta_{w}^{2} \sin ^{2} \mu_{w} \\
\frac{1}{\beta_{w}} \sin 2 \mu_{w} & \frac{1}{\beta_{w}^{2}} \sin ^{2} \mu_{w} & \cos ^{2} \mu_{w}
\end{array}\right]\left[\begin{array}{c}
\alpha_{1}^{x} \\
\beta_{1}^{x} \\
\gamma_{1}^{x}
\end{array}\right]
$$

and

$$
\left[\begin{array}{c}
\alpha_{2}^{y} \\
\beta_{2}^{y} \\
\gamma_{2}^{y}
\end{array}\right]=\left[\begin{array}{ccc}
\cos 2 \mu_{w} & \frac{1}{2 \beta_{w}} \sin 2 \mu_{w} & -\frac{1}{2} \beta_{w} \sin 2 \mu_{w} \\
-\beta_{w} \sin 2 \mu_{w} & \cos ^{2} \mu_{w} & \beta_{w}^{2} \sin ^{2} \mu_{w} \\
\frac{1}{\beta_{w}} \sin 2 \mu_{w} & \frac{1}{\beta_{w}^{2}} \sin ^{2} \mu_{w} & \cos ^{2} \mu_{w}
\end{array}\right]\left[\begin{array}{c}
\alpha_{1}^{y} \\
\beta_{1}^{y} \\
\gamma_{1}^{y}
\end{array}\right]
$$

A reasonable location in a storage ring for a helical wiggler will be in a matched insertion. If the insertion is symmetrical (which is usually the case) and the wigglex is placed symmetrically around the insertion center, then $\alpha_{2}^{\mathrm{x}}=-\alpha_{1}^{\mathrm{x}}, \beta_{2}^{\mathrm{x}}=\beta_{1}^{\mathrm{x}}, \gamma_{2}^{\mathrm{x}}=\gamma_{1}^{\mathrm{x}}, \alpha_{2}^{\mathrm{y}}=-\alpha_{1}^{\mathrm{y}}, \beta_{2}^{\mathrm{y}}=\beta_{1}^{\mathrm{y}}, \gamma_{2}^{\mathrm{y}}=\gamma_{1}^{\mathrm{y}}$ and one can solve for all of the $\alpha^{\prime} s, \beta^{\prime}$ s and $\gamma^{\prime} s$. 
For electron energies in the GeV region and a wiggler length of a few meters, $\mu_{w} \ll 1$ and one can approximate the three by three matrix by:

$\left[\begin{array}{ccc}1 & \mu_{w} / \beta_{w} & -L_{w} \\ -2 L_{w} & 1 & 0 \\ 2 \mu_{w} / \beta_{w} & 0 & 1\end{array}\right]$

leading to

$$
\begin{array}{ll}
\alpha_{1}^{\mathrm{x}}=\alpha_{2}^{\mathrm{x}}=0 & \alpha_{1}^{\mathrm{y}}=\alpha_{2}^{\mathrm{y}}=0 \\
\beta_{1}^{\mathrm{x}}=\beta_{2}^{\mathrm{x}}=\beta_{\mathrm{w}} & \beta_{1}^{\mathrm{y}}=\beta_{2}^{\mathrm{y}}=\beta_{\mathrm{w}} \\
\gamma_{1}^{\mathrm{x}}=\gamma_{2}^{\mathrm{x}}=1 / \beta_{\mathrm{w}} & \gamma_{1}^{\mathrm{y}}=\gamma_{2}^{\mathrm{y}}=1 / \beta_{\mathrm{w}}
\end{array}
$$

It should be noted that $\beta_{w}=\sqrt{2} \rho$. For $2-\mathrm{GeV}$ electrons and $B_{0}=5.5 \mathrm{kG}$, $\rho=12.1 \mathrm{~m}$ and $B_{w}=17.1 \mathrm{~m}$. The rms radial emittances of $2-\mathrm{GeV}$ electron beams can be as low as $1.5 \times 10^{-8} \mathrm{~mm} \cdot \mathrm{rad}$ if special care is taken in the design of the storage ring. ${ }^{7}$ one then gets an rms beam width of about $0.5 \mathrm{~nm}$ in the helical wiggler and a $1-\mathrm{cm}$ bore will yield adequately long quantum lifetime. Furthermore, the horizontal angular divergence $\theta_{\mathrm{r}}$ will be $3.0 \times 10^{-5}$ radians. This easily satisfies the condition 4 that $\theta_{r}$ must be less than $1 /(\sqrt{N Y})$ to maintain spectral purity. Here $\mathrm{N}$ is the number of wiggler periods (of the order of $10^{2}$ ).

Computer runs following orbits for 100 revolutions in a storage ring containing an optically matched helical wigglex confirm that stability can be achieved, provided the proper kicks are given to the beam at the input and output of the wiggler.

The radius of the helical path of off-momentum particles is shifted by only a very small amount, $r_{o} \Delta p / p$. This makes it necessary for the local value of the momentum dispersion function of the storage ring to be zero at both ends of the 
wiggler. With such a configuration it can easily be shown that the effect of a helical wiggler on the relative amount of longitudinal and radial damping is negligible.

\section{Conclusion}

An analysis has been given which yields approximate orbits of electrons in helical wigglers. There seems to be reason to expect that satisfactory performance can be achieved when the helical wiggler is incorporated in an electron storage ring. However, strong coupling between the horizontal and vertical motion in the storage ring is to be expected resulting in increased vertical beam size. Further investigation is essential of the nonlinear effect due to helical wigglers. 
It will be assumed that the field has a sinusoidal distribution in the axial direction, the distribution having the same period as the helical winding. It will be assumed further that no higher harmonics are present. The field pattern can then be represented by

$$
\begin{aligned}
& { }_{B_{r}}=F(r) \sin (\theta-k z) \\
& B_{\theta}=G(x) \cos (\theta-k z)
\end{aligned}
$$

where $2 \pi / k$ is the axial distance in which the field makes a complete revolution. Substitution of these expressions in Maxwel1's equations and elimination of $B_{z}$ by second differentiation reveals the fact that $\mathrm{rg}$ is proportional to $\mathrm{I}_{1}(\mathrm{kr})$, a first order Bessel function of imaginary argument. Finally we obtain:

$$
\begin{aligned}
& B_{r}=2 B_{0}\left\{I_{0}(k x)-\frac{1}{k r} I_{1}(k x)\right\} \sin (\theta-k z) \\
& B_{\theta}=\frac{2 B_{0}}{k r} I_{1}(k r) \quad \cos (\theta-k z) \\
& B_{z}=-2 B_{0} I_{1}(k x) \quad \cos (\theta-k z),
\end{aligned}
$$

where $B_{0}$ is the transverse field amplitude at the axis of the helical winding.

Near the axis the fields can be represented by approximate expressions using the leading terms in the series representing the Bessel functions. For kr less than about 0.8 the field components can be represented with errors less than $1 \%$ by

$$
\begin{aligned}
& B_{r}=B_{0}\left(1+3 k^{2} r^{2} / 8\right) \sin (\theta-k z) \\
& B_{\theta}=B_{0}\left(1+k^{2} r^{2} / 8\right) \cos (\theta-k z) \\
& B_{z}=-k_{0} r\left(1+k^{2} r^{2} / 8\right) \cos (\theta-k z)
\end{aligned}
$$


In rectangular coordinates the field components are:

$$
\begin{aligned}
& B_{x}=-B_{0}\left\{\left(1+k^{2}\left(3 x^{2}+y^{2}\right) / 8\right) \sin k z-\left(k^{2} x y / 4\right) \cos k z\right\} \\
& B_{y}=B_{0}\left\{\left(1+k^{2}\left(x^{2}+3 y^{2}\right) / 8\right) \cos k z-\left(k^{2} x y / 4\right) \sin k z\right\} \\
& B_{z}=-B_{0}\left(1+k^{2}\left(x^{2}+y^{2}\right) / 8\right)(x \cos k z+y \sin k z)
\end{aligned}
$$

Outside of the helical winding the field expressions will be similar but, in order that the fields vanish at infinity, it will be necessary to replace the I functions with $\mathrm{k}$ functions. The field amplitude outside will be obtained by matching the radial component of $B$. This will introduce into all components a factor

$$
A=\frac{\pi}{2} \frac{I_{0}(k a)-\frac{1}{k a} I_{l}(k a)}{K_{0}(k a)+\frac{1}{k a} K_{1}(k a)}
$$

where a is the radius of the winding which, for the moment, we assume to be infinitesimal in thickness.

The current distribution can be derived from the discontinuity in $B_{\theta}$ and $B_{Z}$

$$
\begin{aligned}
& I_{\theta}=-\frac{2 B_{0}}{4 \pi \mu_{0}}\left\{\frac{2 A}{\pi} K_{1}(k a)+I_{1}(k a)\right\} \cos (\theta-k z) \\
& I_{z}=\frac{2 B_{0}}{4 \pi \mu_{0} k_{a}}\left\{\frac{2}{\pi} K_{1}(k a)+I_{1}(k a)\right\} \cos (\theta-k z) .
\end{aligned}
$$

The total current is, with the Wronskian relation,

$$
I=\frac{5 B_{0}}{\pi} \frac{\left(1+1 /\left(k^{2} a^{2}\right)\right)^{1 / 2}}{\left(k_{a} K_{o}(k a)+K_{1}(k a)\right)} \cos (\theta-k z)
$$


Here $I$ is given in amperes per centimeter (axial) and $B_{0}$ is in gauss. The total current in one turn of the helix will be given by setting $\theta=0$ and by integrating (A6) from $\mathrm{kz}=-\pi / 2$ to $+\pi / 2$ to obtain

$$
I_{t o t a l}=\frac{5 \lambda_{o}^{B} o}{\pi^{2}} \frac{\left(1+1 /\left(k^{2} a^{2}\right)\right)^{1 / 2}}{\left(k_{0}(k a)+k_{1}(k a)\right.}
$$

where $\lambda_{0}(=2 \pi / k)$ is the pitch of each helix in centimeters.

Equation (A7) is to be compared with Kincaid's Eq. (1). The two expressions differ by a factor $(4 / \pi)\left(1+1 /\left(k^{2} a^{2}\right)\right)^{\frac{1}{2}}$; this small difference is attributable to the fact that this treatment relates to a distributed winding whereas Kincaid's helices are single wires of infinitesimal cross section.

Equation (A7) is plotted in a semilog plot in Fig. 1. It is evident from the plot that, above $a / \lambda_{0}=0.2$, the expression for $I_{\text {total }} / \lambda_{0} B_{0}$ can be represented by an exponential:

$$
\frac{\mathrm{I}_{\text {total }}}{\lambda_{\mathrm{o}} \mathrm{B}_{\mathrm{o}}}=0.246 \mathrm{e}^{5.68 \mathrm{a} / \lambda_{\mathrm{o}}} \quad \text { amperes } / \mathrm{cm} \cdot \text { gauss }
$$

Using expression (AB) it is possible to analyze windings of finite thickness. We assume that the axially simusoidal current distribution is replaced by a block of current of uniform density, $I_{0} A / \mathrm{cm}^{2}$ and of axial width $\lambda_{0} / 3$. Near the axis, this will. give fields which are to a good approximation the same as those provided by the sinusoidal distribution.

The total current in a block of infinitesimal thickness da at radius a will be $I_{0}\left(\lambda_{0} / 3\right) d a$; it will provide a transverse field $d B_{0}$ on the axis given by (A8)

$$
\mathrm{dB}_{\mathrm{o}}=1.355 \mathrm{I}_{\mathrm{o}} \mathrm{e}^{-5.68 \mathrm{a} / \lambda_{\mathrm{o}} \mathrm{da}}
$$

For a coil of finite thickness, having inner and outer radii $a_{1}$ and $a_{2}$ we integrate (A9) to obtain 


$$
B_{0}=0.2385 I_{0} \lambda_{0}\left(e^{-5.68} a_{1} / \lambda_{0}-e^{-5.68 a_{2} / \lambda_{o}}\right) \text { gauss }
$$

For coils having $a_{1} / \lambda_{0}$ greater than 0.2 , Eq. (A10) can be used to establish the current density $I_{0}$ in amperes per square centimeter required to produce a transverse magnetic flux density of $B_{0}$ gauss in a helix of given pitch and inner and outer dimensions.

\section{Acknowledgments}

The authors axe indebted for helpful discussions and comments to

B. M. Kincaid, J. M. J. Madey, G. K. Green, R. L. Gluckstern, and H. Alan Schwettman. 
T.ABLE I

Magnetic Fields Achievable in Wigglers

$\begin{array}{lcc}\frac{\lambda_{0}(\mathrm{~cm})}{1} & \frac{\mathrm{B}_{\mathrm{O}}\left(\mathrm{I}_{\mathrm{O}}=50,000 \mathrm{~A} / \mathrm{cm}^{2}\right) G}{700} & \frac{\mathrm{B}_{\mathrm{O}}\left(\mathrm{I}_{\mathrm{O}}=100,000 \mathrm{~A} / \mathrm{cm}^{2}\right) G}{1.400} \\ 1.5 & 2700 & 5400 \\ 2 & 5800 & 11,530 \\ 2.5 & 9600 & 19,300\end{array}$




\section{References}

1. Luis R. Elias, William M. Fairbank, John M. J. Madey, H. Alan Schwettman, and Todd I. Smith, Phys. Rev. Letters 36, 717 (1976).

2. H. Motz, J. App1. Phys. 22, 527 (1951), and 24, 826 (1953).

3. John M. J. Madey, J. Appl. Phys. 42, 1906 (1971) .

4. Brian M. Kincaid, J. Appl. Phys. (this issue).

5. W. R. Smythe, Static and Dynamic Electricity (McGraw-Hi11, 1950), p. 277.

6. E. D. Courant and H. S. Snyder, Ann. Phys. 3, 1 (1958).

7. Proposal for a Synchrotron Radiation Source at the Brookhaven National

Laboratory (unpublished). 


\section{Figure Captions}

Fig. 1. Current $\mathrm{I}_{\text {total }}$ per turn required to produce an axial transverse field $B_{o}$ in a helix of pitch $\lambda_{o}$ as a function of the ratio of helix radius to pitch.

Fig. 2. Orbit in a helical wiggler with zero initial coordinates and transverse velocities.

Fig. 3. The functions $u$ and $v$ in a very long wiggler.

Fig. 4. Orbits with almost complete correction of the y motion. 


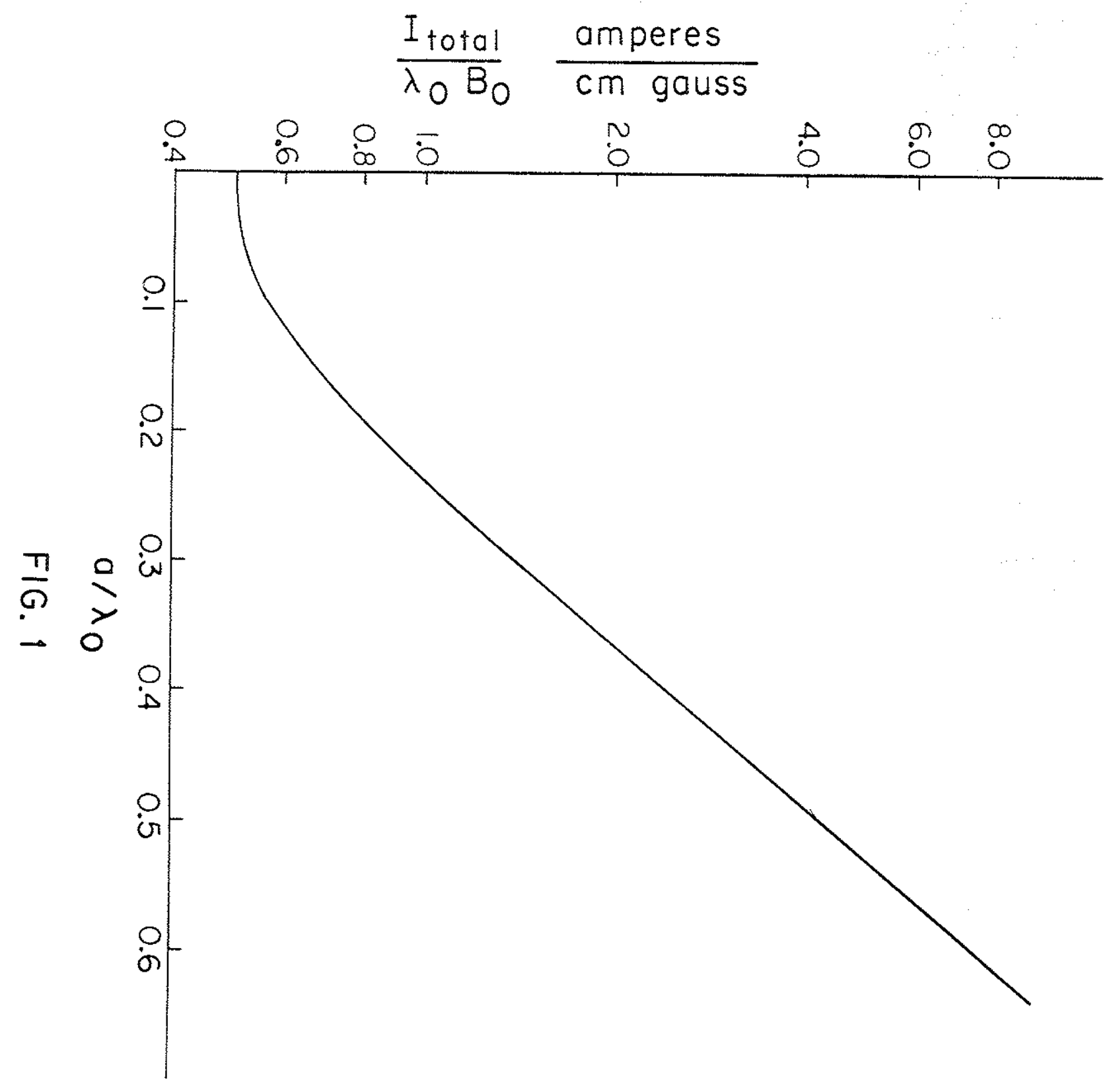




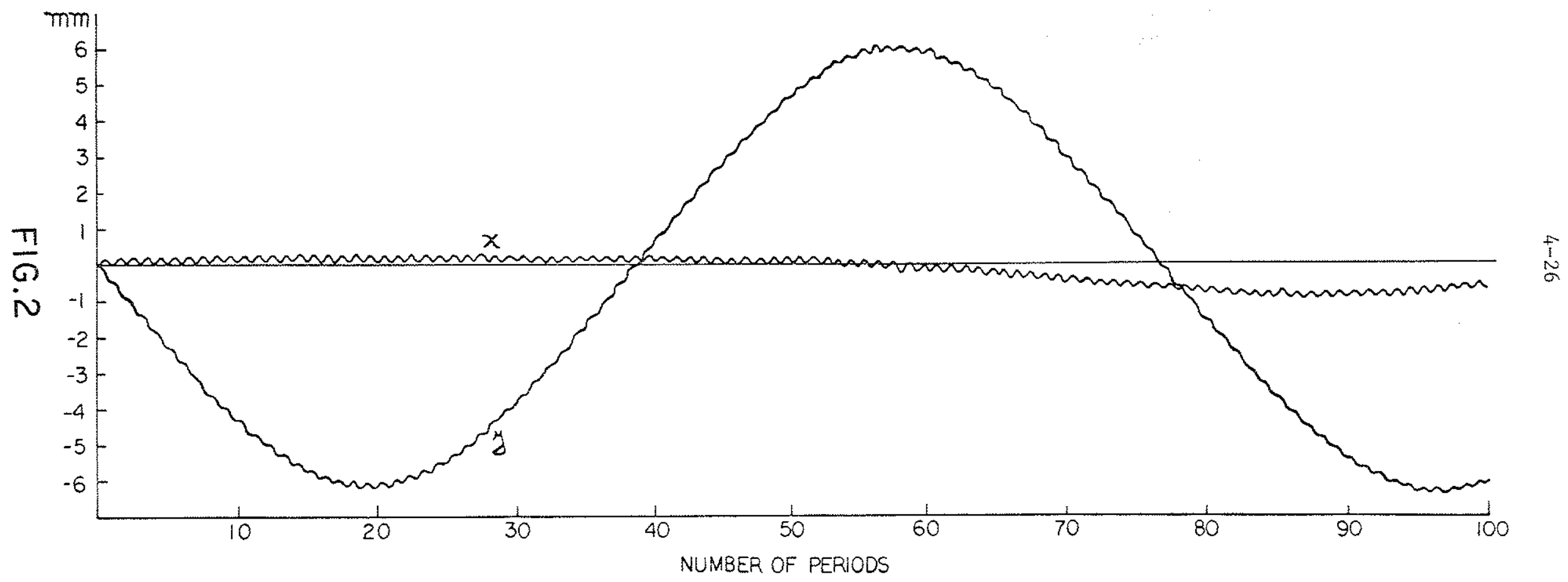




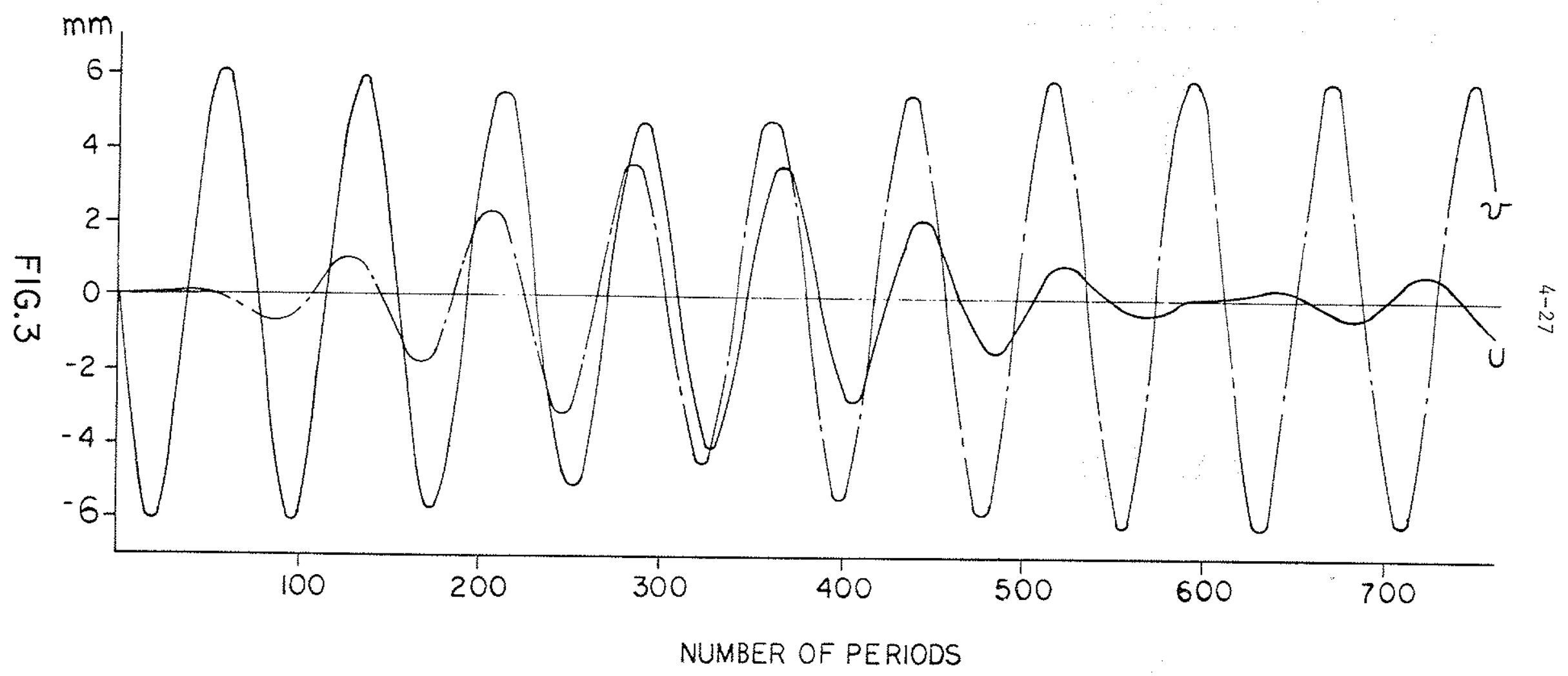




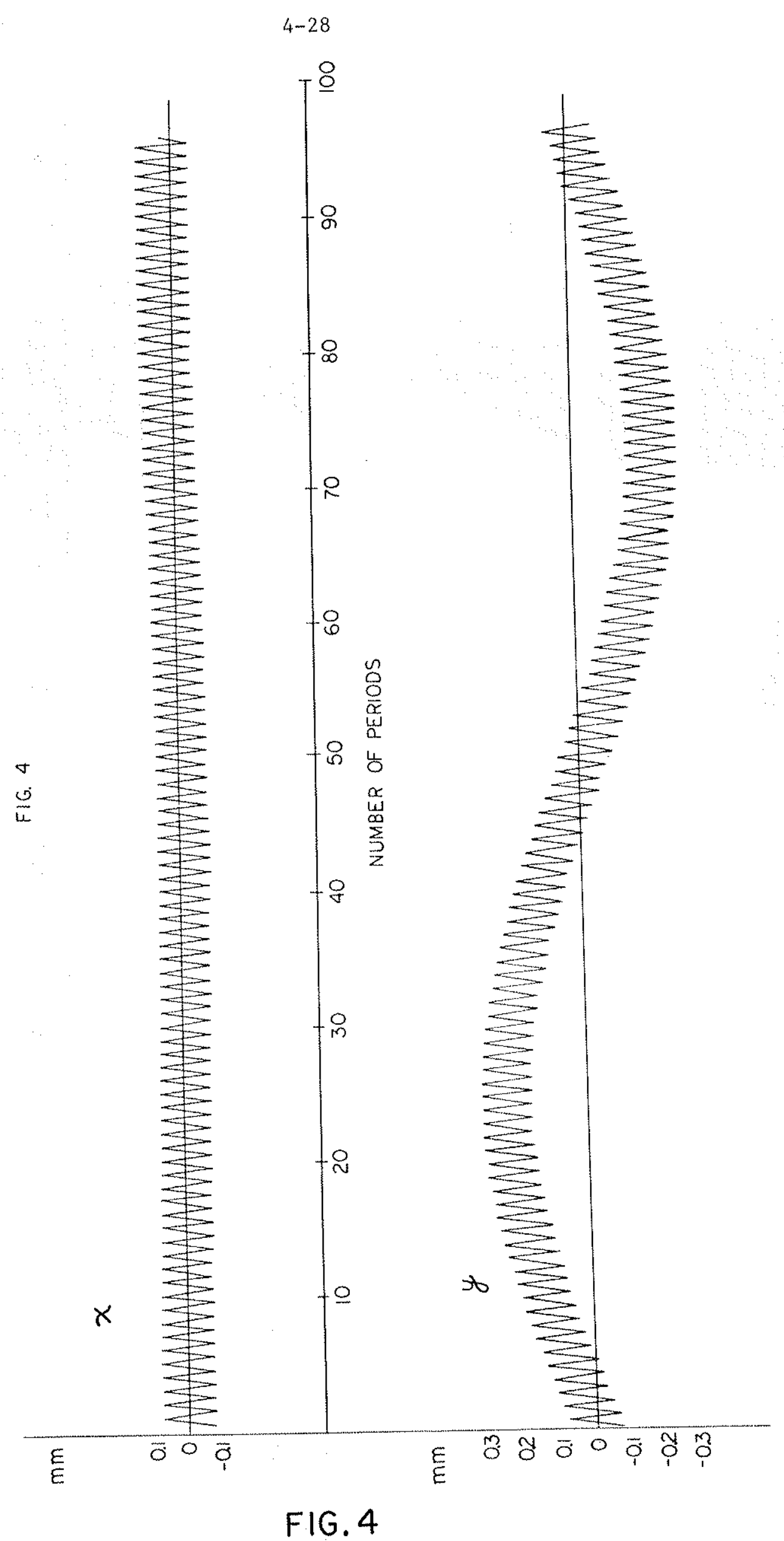

